\title{
(Patho)physiology of gut wall integrity in health and disease in man
}

Citation for published version (APA):

Derikx, J. P. M. (2009). (Patho)physiology of gut wall integrity in health and disease in man. [Doctoral Thesis, Maastricht University]. Universiteit Maastricht. https://doi.org/10.26481/dis.20090612jd

Document status and date:

Published: 01/01/2009

DOI:

10.26481/dis.20090612jd

Document Version:

Publisher's PDF, also known as Version of record

\section{Please check the document version of this publication:}

- A submitted manuscript is the version of the article upon submission and before peer-review. There can be important differences between the submitted version and the official published version of record.

People interested in the research are advised to contact the author for the final version of the publication, or visit the DOI to the publisher's website.

- The final author version and the galley proof are versions of the publication after peer review.

- The final published version features the final layout of the paper including the volume, issue and page numbers.

Link to publication

\footnotetext{
General rights rights.

- You may freely distribute the URL identifying the publication in the public portal. please follow below link for the End User Agreement:

www.umlib.nl/taverne-license

Take down policy

If you believe that this document breaches copyright please contact us at:

repository@maastrichtuniversity.nl

providing details and we will investigate your claim.
}

Copyright and moral rights for the publications made accessible in the public portal are retained by the authors and/or other copyright owners and it is a condition of accessing publications that users recognise and abide by the legal requirements associated with these

- Users may download and print one copy of any publication from the public portal for the purpose of private study or research.

- You may not further distribute the material or use it for any profit-making activity or commercial gain

If the publication is distributed under the terms of Article $25 \mathrm{fa}$ of the Dutch Copyright Act, indicated by the "Taverne" license above, 


\section{(Patho)physiology of gut wall integrity in health and disease in man}

Joseph Petrus Marie Derikx 


\section{Layout and Design:}

D\&L graphics

Print:

Drukkerij Bykorf

ISBN:

978-90-8590-034-4 


\title{
(Patho)physiology of gut wall integrity in health and disease in man
}

\author{
Proefschrift
}

ter verkrijging van de graad van doctor aan de Universiteit van Maastricht op gezag van de Rector Magnificus, Prof. Mr. G.P.M.F. Mols, volgens het besluit van het College van Decanen,

in het openbaar te verdedigen

op vrijdag 12 juni 2009 om 12.00 uur

door

Joseph Petrus Marie Derikx 


\section{Promotores:}

Prof. dr E. Heineman

Prof. dr. W.A. Buurman

\section{Beoordelingscommissie:}

Prof. dr. M.J.A.P. Daemen (voorzitter)

Prof. dr. H.A. Büller (Erasmus Universiteit Rotterdam)

Prof. dr. M. Miserez (Katholieke Universiteit Leuven)

Prof. dr. L.J.I. Zimmermann

Prof. dr. J.H. Zwaveling 


\section{TABLE OF CONTENTS}

Chapter 1. General introduction of gut wall integrity 9

$\begin{array}{ll}\text { 1.1. Introduction } & 10\end{array}$

1.2. The importance of evaluation of gut wall integrity 11

1.3. The composition of the gut wall 14

1.4. Assessment of gut wall integrity (in man) 17

1.5. Aims to be studied 22

Chapter 2. Development and analysis of markers for human gut 29 wall integrity

2.1. Introduction 30

2.2. A pilot study on the non-invasive evaluation of intestinal 31 damage in celiac disease using I-FABP and L-FABP. Journal of Clinical Gastroenterology, in press.

2.3. Liver manipulation causes hepatocyte injury and precedes systemic inflammation in patients undergoing liver resection.

World Journal of Surgery (2007) 31: 2033-8.

2.4. $\quad$ Urine based detection of intestinal tight junction loss. Journal of Clinical Gastroenterology, in press.

Chapter 3. Analysis of markers for compromised gut wall in patients with overt gut damage and in patients with suspected intestinal disease to distinguish the target disease from other pathologies

3.1. Introduction

3.2. Detection of chemotherapy induced enterocyte toxicity with circulating Intestinal Fatty Acid Binding Protein. Journal of Pediatric Hematology and Oncology (2006) 28: $267-9$.

3.3. Loss of enterocyte mass is accompanied by diminished turnover of enterocytes after myeloablative therapy in haematopoietic stem cell transplant recipients.

Annals of Oncology, (2009) 20: 337-42.

3.4. Does gut damage occur around closure of gastroschisis with bladder herniation?

Submitted. 
3.5. Neutrophil activation products: potential new plasma markers for diagnosis of patients with acute appendicitis. Submitted.

3.6. Urine based detection of intestinal mucosal cell damage in neonates with suspected necrotising enterocolitis. Gut (2007) 56: 1473-5.

3.7. Diagnostic accuracy of new non-invasive markers for diagnosing Necrotizing Enterocolitis: urinary I-FABP, urinary claudin-3 and faecal calprotectin.

Submitted.

Chapter 4. Gut wall integrity loss in critically ill patients and in patients undergoing major (non-abdominal) surgery

4.1. Introduction

4.2. Evidence for intestinal and liver epithelial cell injury in the early phase of sepsis. Shock (2007) 28: 544-8.

4.3. Gut mucosal damage in the early phase of children with meningococcal sepsis.

Critical Care Medicine, in revision.

4.4. Visceral injury and systemic inflammation in patients undergoing extracorporeal circulation during aortic surgery.

Annals of Surgery (2008) 248: 117-25.

4.5. New insight in loss of gut barrier during major nonabdominal surgery.

PLoS ONE (2008) 3: e3954.

Chapter 5. Sequelae of human intestinal ischemia-reperfusion

5.1 . Introduction

5.2. A new model to study intestinal ischemia-reperfusion damage in man.

Submitted.

5.3. Rapid reversal of human intestinal ischemiareperfusion induced damage by shedding of injured enterocytes and reepithelialisation.

PLoS ONE (2008) 3: e3428. 
5.4. Mannose-binding lectin null alleles are associated with 233 preserved epithelial cell integrity following intestinal ischemia-reperfusion in man.

Molecular Immunology, in press.

5.5. Enterocyte shedding and epithelial lining repair 239 following ischemia of the human small intestine attenuates inflammation.

Submitted.

Chapter 6. Summary and discussion 259

Nederlandse samenvatting

Curriculum Vitae

Dankwoord 


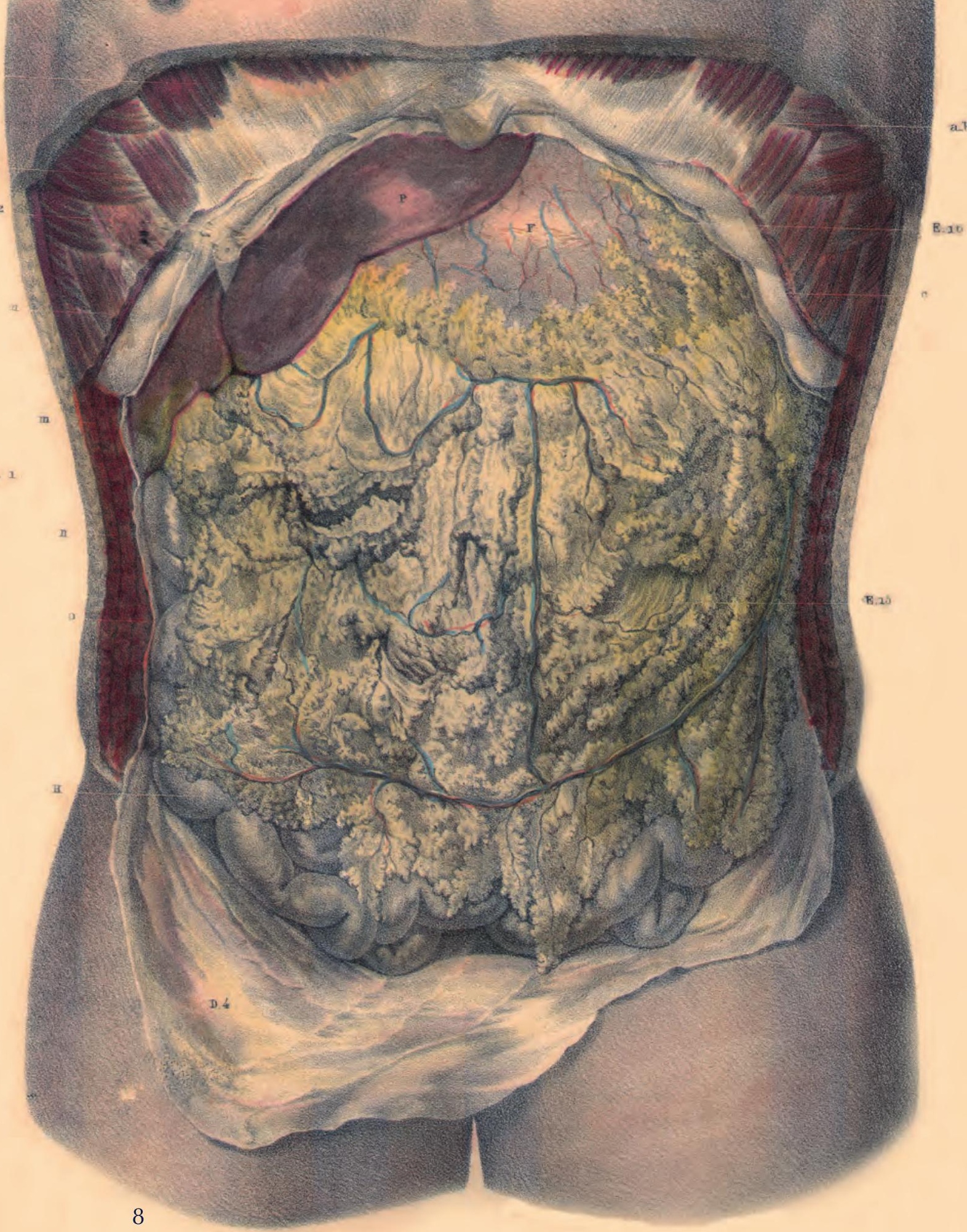




\section{Chapter 1}

\section{General introduction of gut wall integrity}




\section{Chapter 1}

\subsection{INTRODUCTION}

The primary function of the intestine is the absorption of dietary nutrients. To this end, the intestinal epithelium has to be permeable for nutrients and macromolecules important for growth and development. However, the intake of nutrition exposes the epithelium to a wide variety of antigens from food and microbiota and its products ${ }^{1,2}$. The second function of the intestine is therefore to provide an effective barrier to these potentially harmful luminal antigens, toxins and microbiota ${ }^{1,2}$. Different types of (intestinal) pathology, in which failure of gut wall integrity potentially plays an important role, are explicated in paragraph $1.2^{3}$. Several physical/anatomical and immunological barriers have evolved to prevent invasion of foreign or endogenous threats from the intestinal lumen to the sterile interior milieu of the host, which will be discussed in paragraph 1.3. Furthermore, attention is paid to detection methods for the assessment of intestinal barrier loss in man (paragraph 1.4). 


\subsection{The IMPORTANCE OF EVAlUATION OF GUt WALL INTEGRITY}

\section{Early diagnosis and follow-up of intestinal damage in clinical medicine}

Evaluation of intestinal pathology in patients of all age-groups has been a challenge for clinicians. Numerous patients present with abdominal complaints, which are frequently aspecific and therefore correspond to pathologies of most intra- and even some extra-abdominal organs. Laboratory tests and imaging techniques are often helpful in revealing disorders of organs, including the liver, pancreas, heart and kidneys. However, it is currently still difficult to diagnose intestinal pathology in patients presenting with abdominal complaints ${ }^{4,5}$. The current standard technique for assessing intestinal status is endoscopy with bowel biopsy, which is often inadequate as it is invasive, sometimes requires sedation, is expensive and only assesses the function of the biopsied fraction. Moreover, for patients who are neutropenic and/or thrombocytopenic, the procedure is physically hazardous and often ethically unacceptable. Therefore, a major delay in diagnosis is often present in patients with e.g. necrotizing enterocolitis (NEC), chemotherapy-induced mucositis, acute mesenteric ischemia and celiac disease ${ }^{4,6}$. A diagnostic delay results in postponed correct treatment, which is accompanied by higher morbidity and mortality rates. In line with these diagnostic concerns, the follow-up of numerous intestinal diseases is hampered by the absence of non-invasive, rapid diagnostic means to assess intestinal damage for evaluation of the effects of treatment on the recovery of the disorder ${ }^{4}$.

This leads to the following clinical questions:

1) Are proteins, released from cytosol of enterocytes or from tight junctions upon damage, potentially useful as non-invasive markers to diagnose intestinal disease?

2) What is the value of the assessment of such proteins in the follow-up of intestinal disease?

\section{Potential involvement of the gut in the development of postoperative or posttraumatic complications}

Patients undergoing major surgery or sustaining severe trauma are at risk of developing morbidity and mortality from post-operative or post-traumatic systemic inflammatory response syndrome (SIRS), sepsis and multiple organ failure (MOF). The development of such potentially lethal complications in relatively healthy surgical or trauma patients is poorly understood 7,8 . Experimental animal studies have generated the hypothesis that the intestines are central in the origin of post-operative and post-traumatic sequelae ${ }^{9-13}$. 


\section{Chapter 1}

Human studies have contributed insufficiently to gain insight in the applicability of this hypothesis ${ }^{8}$. Recognition of patients at risk of developing post-operative or post-traumatic SIRS, sepsis and MOF is important, since patients with these clinical syndromes have the highest noncardiac mortality rate of patients in the intensive care unit (ICU) ${ }^{9-15}$.

Experimental animal models, resembling the clinical situation of major surgery and trauma, show that haemorrhagic shock leads to disruption of the gut wall integrity, measured by derangement of tight junctions and elevated circulating levels of Fatty Acid Binding Proteins (FABP), originating from damaged intestinal epithelial cells ${ }^{16,17}$. Moreover, leakage of macromolecules, microbial products and microbiota from the intestinal lumen to the circulation and mesenteric lymph nodes, spleen and liver occurs ${ }^{11,17}$. The inflammatory response to microbial products as endotoxin has been reported to be induced via various rapidly induced innate immune mechanisms, ranging from Toll Like Receptors to complement activation ${ }^{18-20}$. Supportive for the importance of the gut in the development of postoperative complications is that protection of the gut wall integrity in haemorrhagic shocked animals by administration of probiotic strains, which inhibit the adhesion of enteric pathogens to intestinal epithelial cells, can result in abrogation of both local and systemic inflammatory responses ${ }^{21}$. However, administration of probiotic strains may also have unwanted side-effects, including disruption of intestinal tight junctions and increase of bacterial translocation ${ }^{22}$.

Studies in patients undergoing major gastro-intestinal, cardiac or vascular surgery, investigating the role of the gut in the development of post-operative complications, are largely restricted to data on increased intestinal permeability for sugars, ${ }^{51} \mathrm{Cr}$-EDTA and the circulatory levels of endotoxin ${ }^{23-29}$. An increased intestinal absorption of sugars or ${ }^{51} \mathrm{Cr}$-EDTA, indirect measures for gut barrier loss, in patients following major surgery support data obtained from animal studies, indicating that the gut barrier is injured after major surgery $24,25,27,29,30$. However, other reports show no changes in intestinal permeability 26,31 . Moreover, the value of measuring gut integrity with the use of sugar probes is argued $^{32,33}$. Similar results are reported on the development of endotoxaemia. Several authors report on increased circulating levels of endotoxin or reduced values of anti-endotoxin antibody during major surgery, suggesting leakage of gut derived bacterial products from the gut lumen into the circulation due to an impaired epithelial barrier, whereas others show unaltered endotoxin concentrations $25,28,34$. Data showing better survival in patients with preoperatively higher anti-endotoxin titres support the theory of intestinal contribution in the development of postoperative complications ${ }^{35}$. In 
conclusion, the debate regarding the involvement of the gut in patients undergoing major surgery is still open.

This leads to the following pathophysiological questions:

1) Is the gut wall integrity impaired in patients undergoing major (nonabdominal) surgery?

2) Does a relationship exist between gut wall integrity loss and the development of post-operative complications in patients?

3) Can these findings be studied in a human model of intestinal ischemiareperfusion? 


\subsection{THE COMPOSITION OF THE GUT WALL}

The gut wall forms a physical/anatomical and immunological barrier. The physical/anatomical barrier of the gut is formed by a monolayer of epithelial cells, originating from multipotent stem cells present in the crypt. The epithelial cells together with the lamina propria form the mucosa of the intestine ${ }^{1,2,36}$. The epithelial stem cells give rise to four major epithelial cells: 1) the absorptive enterocytes, which make up $>80 \%$ of all small intestinal epithelial cells, 2) the goblet cells, which produce a variety of mucins and trefoil peptides, 3) the enteroendocrine cells, which export peptide hormones and 4) the Paneth cells, which secrete a wide variety of antimicrobial peptides (Figure 1).

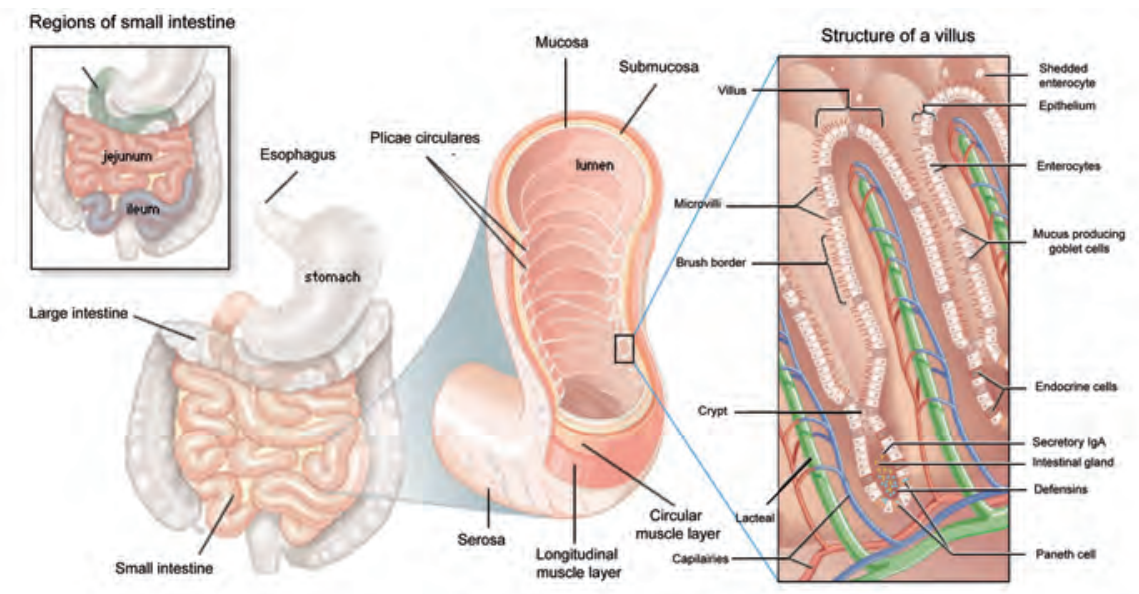

Figure 1: the composition of the small intestine

Tight junctions (TJ) are the major complexes responsible for the adherence of intestinal epithelial cells to each other and are in this context an important part of the intestinal barrier (Figure 2) ${ }^{36,37}$. TJ are built up by multiple proteins: occludins and members of the claudin family are the major sealing proteins. The sealing proteins interact with cytoplasmic proteins, including zonulaoccludin proteins (ZO), functioning as adaptors between the TJ proteins and actin and myosin contractile elements within the cells ${ }^{37}$. Breakdown of this barrier potentially leads to the translocation of luminal antigens, microbiota and their toxic products into the circulation. The layers next to the mucosa, the submucosa, muscularis and serosa, are also of importance, although they are not in direct contact with the lumen. Injury of these layers can result in 
transmural damage of the intestine and lead to a serious complication: passage of the luminal content into the abdominal cavity.

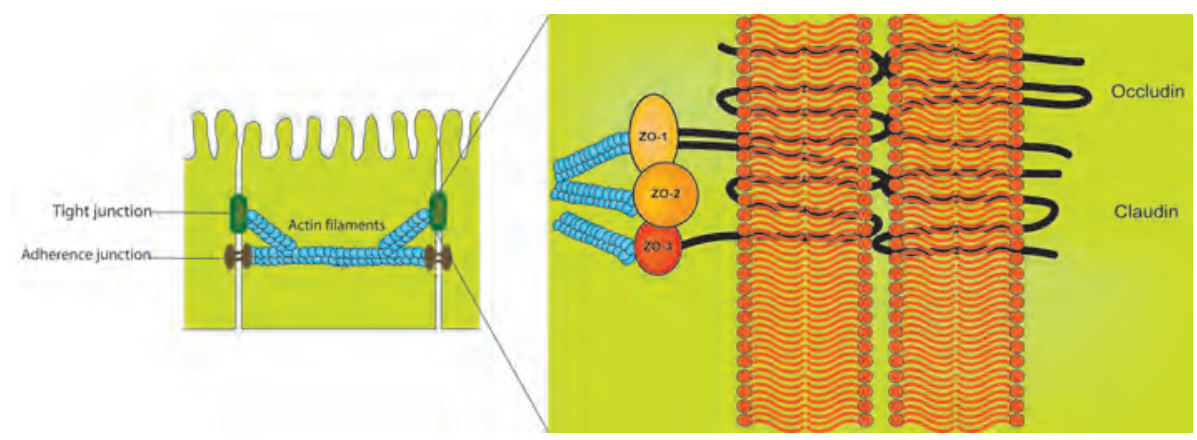

Figure 2: composition of tight-junctions (in green (left) and in detail (right)) between neighbouring enterocytes (in yellow)

Next to the physical/anatomical barrier, there is also an immunological barrier. The intestinal epithelium is not merely a static barrier, but also has a number of specialized protective adaptations ${ }^{1,2}$. Enterocytes are considered to actively participate as innate immune sensors of microbial pathogens and commensal organisms ${ }^{38}$. Host recognition of microbial components is achieved by so-called pattern recognition receptors (PRRs), such as the NOD-like (NLRs) and Toll-like receptors (TLRs). Muramyl dipeptide (MDP), derived from peptidoglycan, is present in the cell wall of virtually all bacteria and recognized by NOD2, a PRR expressed in intestinal epithelial cells ${ }^{38}$ including Paneth cells ${ }^{39}$. Paneth cells secrete defensins, antimicrobial peptides, in the villous crypt, maintaining its sterility. Moreover, we recently reported that Paneth cells are equipped with the proper molecules to recognize and signal endotoxin, one of the most potent immunostimulatory products derived from Gram-negative bacteria (own unpublished results). Continuous antimicrobial protection of the crypt is of crucial importance, since the pluripotent stem cells are located there. Damage to stem cells would have severe consequences for the maintenance of the homeostasis of normal gut epithelium ${ }^{40}$. Goblet cells secrete mucus, a composition of glycoproteins and water, which provides a filter overlying the intestinal epithelium. Additionally goblet cells secrete trefoil peptides, small proteins needed for epithelial growth and repair. Furthermore, gut-associated lymphoid tissue (GALT) is present in the lamina propria and provides immune surveillance. Sampling of luminal antigens occurs by $\mathrm{M}$-cells and dendritic cells (DC), which present antigens to $\mathrm{T}$ and $\mathrm{B}$ cells, thereby inducing an effector 


\section{Chapter 1}

immune response. This response includes secretion of large amounts of IgA by plasma cells. This secretory IgA covers the mucosal surface and has a major role in excluding antigen from passing the epithelium ${ }^{1,2}$. 


\subsection{ASSESSMENT OF GUt WALL INTEGRITY (IN MAN)}

Plasma/serum, urinary and faecal markers are currently available as useful tools to study the condition of the gut wall in man. In this paragraph a brief description of these tests is provided analogue to the previous described physical/anatomical (I) and the immunological (II) components of the gut wall.

\section{Physical/anatomical components of the gut wall}

\section{la. Condition of enterocytes}

Measurement of endogenous cytosolic enterocyte proteins in urine or plasma have been shown to be useful to estimate enterocyte damage. Fattty Acid Binding Proteins (FABP) comprise a class of low molecular weight (14-15 kDa) cytosolic proteins found in high concentrations in tissues involved in the uptake and consumption of fatty acids. Intestinal Fatty Acid Binding Protein (I-FABP) is primarily limited to mature enterocytes of the small and large intestine ${ }^{41,42}$. It circulates in low amounts in the blood stream of healthy individuals. I-FABP is a useful plasma marker for early enterocyte cell death and levels rise rapidly after episodes of acute intestinal ischaemia and inflammation ${ }^{41-43}$. The level of circulating I-FABP has been reported to correlate with the histological status of the epithelium after intestinal ischaemia-reperfusion in experimental studies ${ }^{44-46}$. A second gut-specific FABP is Ileal-Bile Acid Binding Protein (I-BABP), which is exclusively present in mature enterocytes of the jejunum and ileum ${ }^{47}$. Enterocytes also contain Liver-FABP (L-FABP), which is localized in small amounts in the mature enterocytes of the small and large intestine but in abundance in the liver ${ }^{41}$. Next to FABP, Glutathione S-Transferases (GST) are a family of more or less tissue specific cytosolic enzymes. These proteins are involved in the detoxification of a range of xenobiotic compounds by conjugation to glutathione and grouped into species-dependent families based on their isoelectric point ${ }^{48}$. Alpha and pi GST are found in the small and large intestine ${ }^{49}$. However, they are not organ-specific, since they are also expressed in liver and kidney ${ }^{50}$. Plasma levels of alpha GST are elevated upon ischemic intestinal damage ${ }^{51}$. In conclusion, I-FABP and I-BABP are most promising endogenous enterocyte proteins (markers) to assess enterocyte injury, since these proteins are specifically expressed in the gut and released immediately into the circulation upon cell damage.

\section{Ib. Functional enterocyte mass}

Functional enterocyte mass is reflected by levels of circulating citrulline, an amino acid not incorporated into proteins ${ }^{52-54}$. Differentiated small intestinal enterocytes specifically produce citrulline from glutamine and are responsible 


\section{Chapter 1}

for the major part of the total amount of circulating citrulline ${ }^{54,55}$. Declined circulating levels of citrulline provide evidence for the loss of small bowel epithelial cell mass, as is shown in haemopoietic stem cell transplant recipients suffering from severe oral and gastrointestinal mucositis following intensive myeloablative therapy ${ }^{52-54}$.

\section{Ic. Condition of tight junctions}

Currently, invasive intestinal biopsies provide the only possibility to detect tight junction breakdown. Endoscopy for biopsy is a time-consuming and invasive procedure.

\section{Id. Intestinal permeability}

Intestinal permeability is frequently assessed using oral ingestion of various relatively small to large-sized probe molecules and measurement of their urinary excretion ${ }^{3,8,32}$. Large molecules are thought to traverse the epithelium by paracellular pathways via tight junctions between the enterocytes. Permeability of the tight junctions presumably increases in diseased or damaged mucosa, resulting in increased absorption of large molecules. Small molecules are postulated to predominantly pass by transcellular pathways through aqueous pores in the enterocyte membranes that are too small to permit the passage of large molecules. The ratio of urinary excretion of the relatively large molecule is compared with that of the relatively small molecule. When a large and small molecule are combined in the test solution at a fixed concentration ratio, the effects of variables, such as gastric emptying, intestinal transit time, and renal clearance will apply equally to both. Thus, the urinary excretion ratio between these two molecules is expected to be only influenced by the difference in gut permeability for each molecule.

Disaccharides (lactulose) or Poly-ethylene-glycol (PEG)-3350 are frequently used as orally ingested large molecules and monosaccharides (mannitol, Lrhamnose) or PEG-400 are used as small molecular probes ${ }^{32}$. Subsequently, the renal excretion of the two probes is monitored over a defined interval (mostly 5 hours), and permeability is then expressed as the quotient (ratio) of the urinary recovery of the large molecule divided by the small molecule ${ }^{32}$. It is assumed that the used probes are non-fermentable by bacteria in the gastrointestinal lumen and that they are not metabolized in the body. These molecules are also supposed to be excreted in urine in proportion to the amount that has been absorbed through the intestinal mucosa ${ }^{32}$. Thus far, contrasting results have been reported for intestinal permeability tests using dual probe molecules in several studies ${ }^{8,33}$. This is mainly attributed to a number of assumptions that have to be made to 
simplify the interpretation of these tests ${ }^{32,33}$. Especially pathways of intestinal permeation of the different molecules and the mechanism by which permeability is altered are as yet incompletely understood.

\section{le. Translocation of bacteria and their products}

Breakdown of the mucosal barrier potentially leads to translocation of microbiota or their toxic products. Two promising plasmamarkers, reflecting translocation of bacteria or their products, are D-lactate and endotoxin (lipopolysaccharide, LPS), which are metabolic products or components of the commensal bacteria of the gastrointestinal tract. D-lactate is only produced by bacteria as a product of bacterial fermentation ${ }^{56}$. Baseline levels of D-lactate in healthy subjects are very low, but the exact mechanism by which D-lactate enters the bloodstream is unknown. Increased levels of D-lactate have been correlated with conditions in which the number of bacteria elevates rapidly, including in patients with bacterial overgrowth due to infection, short bowel syndrome and mesenteric ischaemia ${ }^{57,58}$. LPS, the major constituent of the outer membrane of Gram-negative bacteria, is released from bacteria when replicating or dying ${ }^{59}$. Increased circulating LPS levels have been related to an impaired mucosal barrier. The presence of LPS can be measured directly in blood, e.g. by the Limulus Amoebocyte Lysate (LAL) assay ${ }^{60}$. In addition, antiLPS antibodies can be measured by endotoxin-core antibody (EndoCAb), an indirect measurement of LPS leakage into the circulation ${ }^{61}$. A drop in levels of circulating anti-LPS antibodies is considered to indicate consumption of antibodies to LPS by exposure to LPS ${ }^{27}$.

\section{If. Transmural damage}

Any part of the gastro-intestinal tract may undergo damage to all layers of the GI wall from a variety of causes, releasing gastric or intestinal contents into the peritoneal cavity, which potentially cause peritonitis. Symptoms develop suddenly, with severe pain followed shortly by signs of (septic) shock. If a perforation is noted, immediate surgery is necessary, because mortality from peritonitis increases rapidly. The diagnosis of transmural damage (i.e. perforation) of a gastro-intestinal organ usually depends on the detection of free intraperitoneal air, which is most often located in the right subphrenic space. Traditionally, a chest X-ray and a plain abdominal X-ray in the upright position, or, more recently, ultrasonography are the diagnostic tools used to detect free air. However, the sensitivity of these tools is $<80 \%$. Currently, a computed tomography (CT) scan is sometimes performed, which may detect free intraperitoneal air as well as small fluid collections and subtle tissue-infiltration at different locations. 


\section{Chapter 1}

\section{Ig. Splanchnic perfusion}

Numerous clinical conditions are accompanied by a reduced splanchnic blood flow, including vascular disease, major surgery and various types of shock. Prolonged hypoperfusion of the splanchnic region will inevitably lead to hypoxic tissue injury. Furthermore, the splanchnic region is an important source and target of inflammatory mediators, which have a major impact on both systemic and regional blood flow and tissue function ${ }^{62}$. Gut mucosal perfusion can be invasively measured by gastric tonometry. Gastric tonometry assesses the $\mathrm{pCO}_{2}$ in the gastric mucosa, taking into account that an increase in tissue $\mathrm{CO}_{2}$ production accompanies anaerobic metabolism, signifying the effectiveness of regional splanchnic perfusion ${ }^{63}$.

\section{Immunological components of the gut wall Gut wall inflammation}

A broad range of pathologies can lead to intestinal inflammation: neoplasia, inflammatory bowel disease (IBD), infections, auto-immune diseases (e.g. celiac disease), ischemia-reperfusion, intestinal hypoperfusion, and e.g. the use of non-steroidal anti-inflammatory drugs. Generally, defects or increased permeability of the mucosal barrier will cause intestinal inflammation in response to the enormous number of bacteria present in the bowel. The pathogenesis of inflammatory intestinal diseases implies the recruitment of leukocytes into the intestinal wall ${ }^{64}$. Activated neutrophils infiltrate the mucosa and their products can be detected in faeces due to release into the intestinal lumen. Obviously, changes in neutrophil release products can also be detected in plasma/serum, but plasma/serum levels may also be increased by various conditions other than gut inflammation. Therefore, faecal markers of neutrophils are specific for the detection of inflammatory intestinal diseases. Numerous neutrophil derived proteins present in stool have been studied, including calprotectin, lactoferrin and elastase ${ }^{64}$. The most promising marker is calprotectin, because of its remarkable resistance to proteolytic degradation and its stability in stool kept on room temperature for at least 7 days ${ }^{65}$.

Calprotectin, also known as MRP-8/MRP-14 or S100A8/A9 complex, is a $36 \mathrm{kDa}$ calcium and zinc binding protein that plays a regulatory role in the inflammatory process. It constitutes about $60 \%$ of the soluble proteins in human neutrophilic cytosol and is also found in monocytes, macrophages and ileal tissue eosinophils. It is released during cell activation or cell death and has antiproliferative, antimicrobial and immunomodulating functions ${ }^{64,65}$.

Fecal calprotectin is nowadays used in clinical practice to evaluate disease activity in the follow-up of patients treated because of active IBD ${ }^{64}$. It 
was found that faecal calprotectin levels correlated well with endoscopic as well as histological disease activity of patients with IBD ${ }^{64}$. Moreover, recent studies showed that normalization of calprotectin levels in patients with established IBD is a strong indicator of mucosal healing. Furthermore, several studies showed that calprotectin was a very sensitive detection marker of inflammation in patients with inflammatory bowel disease (IBD, Crohn's disease and ulcerative colitis) compared with healthy controls and patients with irritable bowel syndrome (IBS), though not a specific marker as increased levels were also found in neoplasia, infections, and polyps ${ }^{64}$. Bunn et al confirmed these results on calprotectin levels in children with IBD ${ }^{66,67}$. Carroll et al stated that fecal calprotectin might be a useful marker of gastrointestinal mucosal inflammation in neonates in a pilot study comparing seven patients with proven necrotizing enterocolitis (NEC) with seven healthy peers ${ }^{68}$. 


\section{Chapter 1}

\subsection{Aims to Be StUdied}

On the basis of the questions brought forward in paragraph 1.2 concerning the importance of the evaluation of gut wall integrity, the following aims are subject of this thesis.

aim 1: development and analysis of markers for human gut wall integrity, in particular for enterocyte and TJ condition (Chapter 2)

aim 2: analysis of markers for compromised gut wall in patients with overt intestinal damage and in patients with suspected intestinal disease to distinguish the target disease from other pathologies (Chapter 3)

aim 3: investigate the integrity of the gut wall in critically ill patients and in patients undergoing major (non-abdominal) surgery (Chapter 4)

aim 4: clarify the sequelae of intestinal ischemia-reperfusion in a human model (Chapter 5)

\section{REFERENCES}

1. Sansonetti PJ. War and peace at mucosal surfaces. Nat Rev Immunol 2004;4(12):953-64.

2. Nagler-Anderson C. Man the barrier! Strategic defences in the intestinal mucosa. Nat Rev Immunol 2001;1(1):59-67.

3. Arrieta MC, Bistritz L, Meddings JB. Alterations in intestinal permeability. Gut 2006;55(10):1512-20.

4. Dewar DH, Ciclitira PJ. Clinical features and diagnosis of celiac disease. Gastroenterology 2005;128(4 Suppl 1):S19-24.

5. Nikolaus S, Schreiber S. Diagnostics of inflammatory bowel disease. Gastroenterology 2007;133(5):1670-89.

6. Oldenburg WA, Lau LL, Rodenberg TJ, Edmonds HJ, Burger CD. Acute mesenteric ischemia: a clinical review. Arch Intern Med 2004;164(10):1054-62.

7. Russell JA. Management of sepsis. N Engl J Med 2006;355(16):1699-713.

8. Soeters PB, Luyer MD, Greve JW, Buurman WA. The significance of bowel permeability. Curr Opin Clin Nutr Metab Care 2007;10(5):632-8.

9. Rowlands BJ, Soong CV, Gardiner KR. The gastrointestinal tract as a barrier in sepsis. $\mathrm{Br}$ Med Bull 1999;55(1):196-211.

10. Moore FA. The role of the gastrointestinal tract in postinjury multiple organ failure. Am J Surg 1999;178(6):449-53.

11. Rotstein OD. Pathogenesis of multiple organ dysfunction syndrome: gut origin, protection, and decontamination. Surg Infect (Larchmt) 2000;1(3):217-23; discussion 223-5. 
12. Fink MP, Delude RL. Epithelial barrier dysfunction: a unifying theme to explain the pathogenesis of multiple organ dysfunction at the cellular level. Crit Care Clin 2005;21(2):177-96.

13. Clark JA, Coopersmith CM. Intestinal crosstalk: a new paradigm for understanding the gut as the "motor" of critical illness. Shock 2007;28(4):384-93.

14. Anup R, Balasubramanian KA. Surgical stress and the gastrointestinal tract. J Surg Res 2000;92(2):291-300.

15. Lemaire LC, van der Poll T, van Lanschot JJ, et al. Minimally invasive surgery induces endotoxin-tolerance in the absence of detectable endotoxemia. I Clin Immunol 1998;18(6):414-20.

16. Haan Jd, Lubbers T, Hadfoune M, et al. Post-Shock Intervention with High-Lipid Enteral Nutrition Reduces Inflammation and Tissue Damage. accepted by Ann Surg.

17. Yang R, Han X, Uchiyama T, et al. IL-6 is essential for development of gut barrier dysfunction after hemorrhagic shock and resuscitation in mice. Am J Physiol Gastrointest Liver Physiol 2003;285(3):G621-9.

18. Beutler B. Inferences, questions and possibilities in Toll-like receptor signalling. Nature 2004;430(6996):257-63.

19. Quezado ZM, Hoffman WD, Winkelstein JA, et al. The third component of complement protects against Escherichia coli endotoxin-induced shock and multiple organ failure. J Exp Med 1994;179(2):569-78.

20. Strunk RC, Whitehead AS, Cole FS. Pretranslational regulation of the synthesis of the third component of complement in human mononuclear phagocytes by the lipid A portion of lipopolysaccharide. J Clin Invest 1985;76(3):985-90.

21. Ukena SN, Singh A, Dringenberg U, et al. Probiotic Escherichia coli Nissle 1917 Inhibits Leaky Gut by Enhancing Mucosal Integrity. PLoS ONE 2007;2(12):e1308.

22. Luyer MD, Buurman WA, Hadfoune $M$, et al. Strain-specific effects of probiotics on gut barrier integrity following hemorrhagic shock. Infect Immun 2005;73(6):3686-92.

23. Kanwar S, Windsor AC, Welsh F, Barclay GR, Guillou PJ, Reynolds JV. Lack of correlation between failure of gut barrier function and septic complications after major upper gastrointestinal surgery. Ann Surg 2000;231(1):88-95.

24. Ohri SK, Somasundaram S, Koak Y, et al. The effect of intestinal hypoperfusion on intestinal absorption and permeability during cardiopulmonary bypass. Gastroenterology 1994;106(2):318-23.

25. Riddington DW, Venkatesh B, Boivin CM, et al. Intestinal permeability, gastric intramucosal $\mathrm{pH}$, and systemic endotoxemia in patients undergoing cardiopulmonary bypass. Jama 1996;275(13):1007-12.

26. Rossi M, Sganga G, Mazzone M, et al. Cardiopulmonary bypass in man: role of the intestine in a self-limiting inflammatory response with demonstrable bacterial translocation. Ann Thorac Surg 2004;77(2):612-8. 


\section{Chapter 1}

27. Braun JP, Buhner S, Kastrup M, et al. Barrier function of the gut and multiple organ dysfunction after cardiac surgery. J Int Med Res 2007;35(1):72-83.

28. Buttenschoen K, Buttenschoen DC, Berger D, et al. Endotoxemia and acute-phase proteins in major abdominal surgery. Am / Surg 2001;181(1):36-43.

29. Holland J, Carey M, Hughes N, et al. Intraoperative splanchnic hypoperfusion, increased intestinal permeability, down-regulation of monocyte class II major histocompatibility complex expression, exaggerated acute phase response, and sepsis. Am J Surg 2005;190(3):393-400.

30. Doig CJ, Sutherland LR, Sandham JD, Fick GH, Verhoef M, Meddings JB. Increased intestinal permeability is associated with the development of multiple organ dysfunction syndrome in critically ill ICU patients. Am J Respir Crit Care Med 1998;158(2):444-51.

31. Malagon I, Onkenhout W, Klok G, van der Poel PF, Bovill JG, Hazekamp MG. Gut permeability in paediatric cardiac surgery. Br J Anaesth 2005;94(2):181-5.

32. Bjarnason I, MacPherson A, Hollander D. Intestinal permeability: an overview. Gastroenterology 1995;108(5):1566-81.

33. Fink MP. Interpreting dual-sugar absorption studies in critically ill patients: what are the implications of apparent increases in intestinal permeability to hydrophilic solutes? Intensive Care Med 1997;23(5):489-92.

34. Soong CV, Halliday MI, Barclay GR, Hood JM, Rowlands BJ, Barros D'Sa AA. Intramucosal acidosis and systemic host responses in abdominal aortic aneurysm surgery. Crit Care Med 1997;25(9):1472-9.

35. Bennett-Guerrero E, Ayuso L, Hamilton-Davies C, et al. Relationship of preoperative antiendotoxin core antibodies and adverse outcomes following cardiac surgery. Jama 1997;277(8):646-50

36. Snoeck V, Goddeeris B, Cox E. The role of enterocytes in the intestinal barrier function and antigen uptake. Microbes Infect 2005;7(7-8):997-1004.

37. Liu Z, Li N, Neu J. Tight junctions, leaky intestines, and pediatric diseases. Acta Paediatr 2005;94(4):386-93.

38. Hisamatsu T, Suzuki M, Reinecker HC, Nadeau WJ, McCormick BA, Podolsky DK. CARD15/NOD2 functions as an antibacterial factor in human intestinal epithelial cells. Gastroenterology 2003;124:993-1000.

39. Lala S, Ogura Y, Osborne C, et al. Crohn's disease and the NOD2 gene: a role for Paneth cells. . Gastroenterology 2003;125:47-57.

40. Mukherjee S, Vaishnava S, Hooper LV. Multi-layered regulation of intestinal antimicobial defense. Cell Mol Life Sci 2008:1-9.

41. Pelsers MM, Hermens WT, Glatz JF. Fatty acid-binding proteins as plasma markers of tissue injury. Clin Chim Acta 2005;352(1-2):15-35. 
42. Lieberman JM, Sacchettini J, Marks C, Marks WH. Human intestinal fatty acid binding protein: report of an assay with studies in normal volunteers and intestinal ischemia. Surgery 1997;121(3):335-42.

43. Kanda T, Fujii H, Tani T, et al. Intestinal fatty acid-binding protein is a useful diagnostic marker for mesenteric infarction in humans. Gastroenterology 1996;110(2):339-43.

44. Gollin G, Marks C, Marks WH. Intestinal fatty acid binding protein in serum and urine reflects early ischemic injury to the small bowel. Surgery 1993;113(5):545-51.

45. Kanda $\mathrm{T}, \mathrm{Nakatomi} \mathrm{Y}$, Ishikawa $\mathrm{H}$, et al. Intestinal fatty acid-binding protein as a sensitive marker of intestinal ischemia. Dig Dis Sci 1992;37(9):1362-7.

46. Marks WH, Gollin G. Biochemical detection of small intestinal allograft rejection by elevated circulating levels of serum intestinal fatty acid binding protein. Surgery 1993;114(2):206-10.

47. Watanabe K, Hoshi N, Tsuura Y, et al. Immunohistochemical distribution of intestinal 15 kDa protein in human tissues. Arch Histol Cytol 1995;58(3):303-6.

48. Beckett GJ, Hayes JD. Glutathione S-transferases: biomedical applications. Adv Clin Chem 1993;30:281-380.

49. Coles BF, Chen G, Kadlubar FF, Radominska-Pandya A. Interindividual variation and organ-specific patterns of glutathione S-transferase alpha, mu, and pi expression in gastrointestinal tract mucosa of normal individuals. Arch Biochem Biophys 2002;403(2):270-6.

50. Campbell JA, Corrigall AV, Guy A, Kirsch RE. Immunohistologic localization of alpha, mu, and pi class glutathione S-transferases in human tissues. Cancer 1991;67(6):1608-13.

51. Khurana S, Corbally MT, Manning F, Armenise T, Kierce B, Kilty C. Glutathione Stransferase: a potential new marker of intestinal ischemia. I Pediatr Surg 2002;37(11):1543-8.

52. Crenn P, Coudray-Lucas C, Thuillier F, Cynober L, Messing B. Postabsorptive plasma citrulline concentration is a marker of absorptive enterocyte mass and intestinal failure in humans. Gastroenterology 2000;119(6):1496-505.

53. Crenn P, Vahedi K, Lavergne-Slove A, Cynober L, Matuchansky C, Messing B. Plasma citrulline: A marker of enterocyte mass in villous atrophy-associated small bowel disease. Gastroenterology 2003;124(5):1210-9.

54. Curis E, Nicolis I, Moinard C, et al. Almost all about citrulline in mammals. Amino Acids 2005;29(3):177-205.

55. Curis E, Crenn P, Cynober L. Citrulline and the gut. Curr Opin Clin Nutr Metab Care 2007; 10(5):620-6.

56. Smith SM, Eng RH, Buccini F. Use of D-lactic acid measurements in the diagnosis of bacterial infections. J Infect Dis 1986;154(4):658-64.

57. Poeze M, Froon AH, Greve JW, Ramsay G. D-lactate as an early marker of intestinal ischaemia after ruptured abdominal aortic aneurysm repair. Br J Surg 1998;85(9):1221-4. 


\section{Chapter 1}

58. Murray MJ, Gonze MD, Nowak LR, Cobb CF. Serum D(-)-lactate levels as an aid to diagnosing acute intestinal ischemia. Am / Surg 1994;167(6):575-8.

59. Beutler B. Innate immunity: an overview. Mol Immunol 2004;40(12):845-59.

60. Caridis DT, Reinhold RB, Woodruff PW, Fine J. Endotoxaemia in man. Lancet 1972;1(7765):1381-5.

61. Barclay GR. Endogenous endotoxin-core antibody (EndoCAb) as a marker of endotoxin exposure and a prognostic indicator: a review. Prog Clin Biol Res 1995;392:263-72.

62. Takala J. Determinants of splanchnic blood flow. Br J Anaesth 1996;77(1):50-8.

63. Cerny V, Cvachovec K. Gastric tonometry and intramucosal $\mathrm{pH}$--theoretical principles and clinical application. Physiol Res 2000;49(3):289-97.

64. Lundberg JO, Hellstrom PM, Fagerhol MK, Weitzberg E, Roseth AG. Technology insight: calprotectin, lactoferrin and nitric oxide as novel markers of inflammatory bowel disease. Nat Clin Pract Gastroenterol Hepatol 2005;2(2):96-102.

65. Fagerhol MK. Calprotectin, a faecal marker of organic gastrointestinal abnormality. Lancet 2000;356(9244):1783-4.

66. Bunn SK, Bisset WM, Main MJ, Golden BE. Fecal calprotectin as a measure of disease activity in childhood inflammatory bowel disease. J Pediatr Gastroenterol Nutr 2001;32(2):171-7.

67. Bunn SK, Bisset WM, Main MJ, Gray ES, Olson S, Golden BE. Fecal calprotectin: validation as a noninvasive measure of bowel inflammation in childhood inflammatory bowel disease. J Pediatr Gastroenterol Nutr 2001;33(1):14-22.

68. Carroll D, Corfield A, Spicer R, Cairns P. Faecal calprotectin concentrations and diagnosis of necrotising enterocolitis. Lancet 2003;361(9354):310-1. 
General introduction of gut wall integrity 


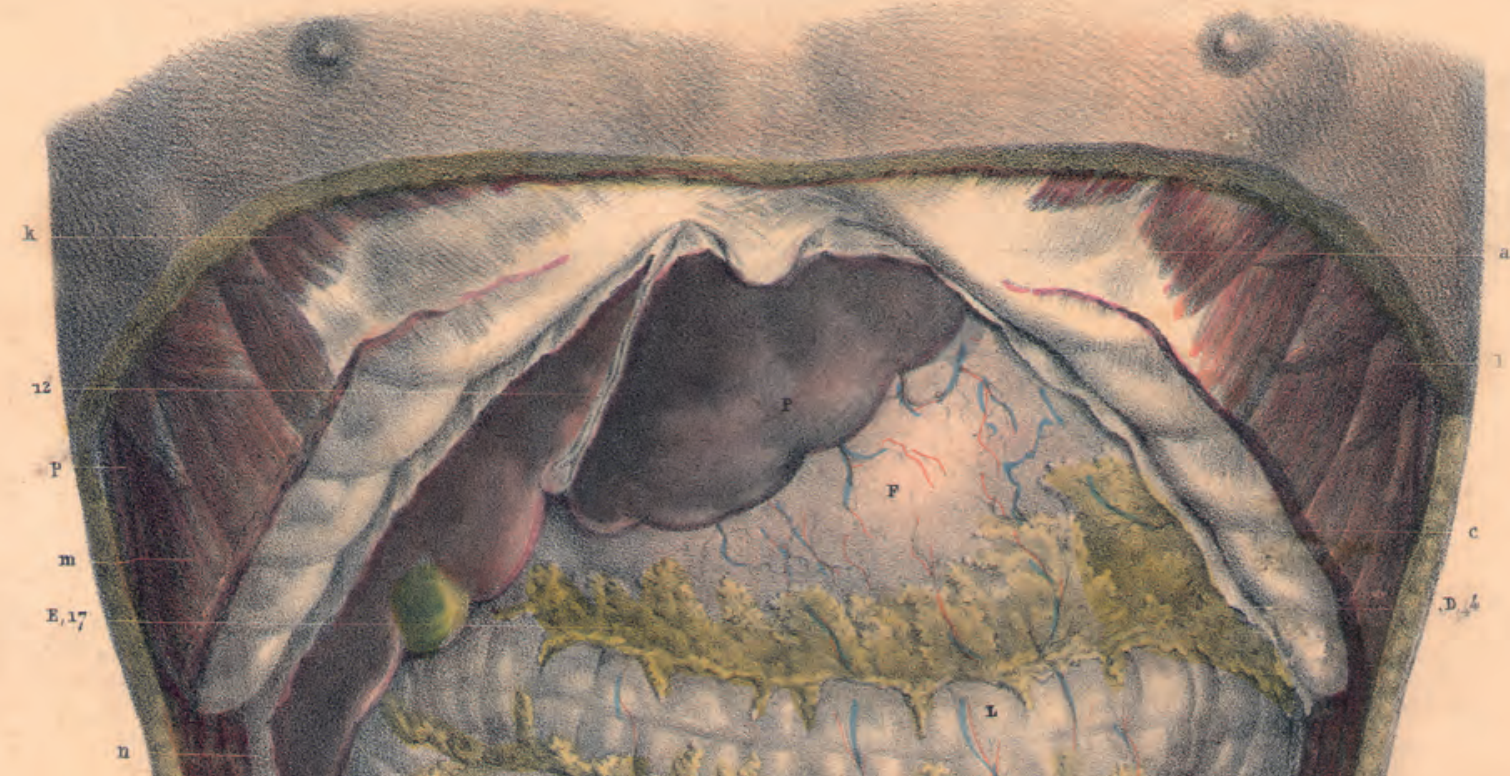

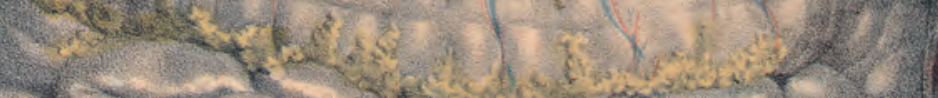

k (

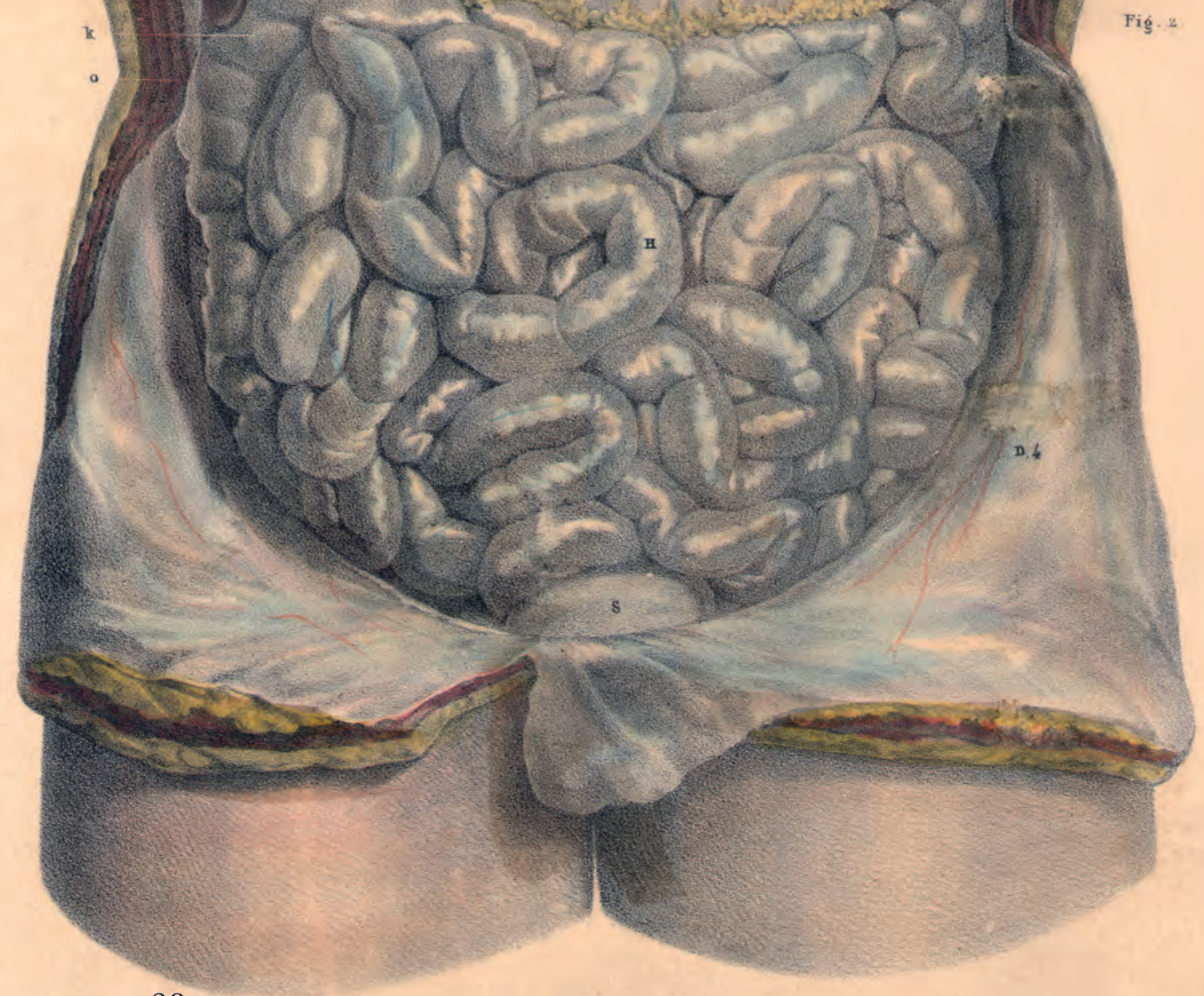




\section{ChAPTER 2}

Development and analysis of markers for human gut wall integrity 


\subsection{INTRODUCTION}

To study the first aim: development and analysis of markers for human gut wall integrity, in particular for enterocyte and TJ condition, attention was paid to the investigation of characteristics of FABP and TJ in human and animal studies. In the first study the distribution and microscopic localization of FABP in the human gastrointestinal tract was analyzed after collection of histological normal human intestinal full thickness tissue during routine surgery (paragraph 2.2). In the same study, the release of FABP was evaluated in patients with apparent intestinal damage, i.e. celiac disease. In the next study, the release and clearance of FABP was studied during surgery by collection of blood from efferent and afferent vessels of the intestine, liver and kidney during surgery (paragraph 2.3). Finally, a potential new marker to analyze the condition of tight junctions was studied in a translational setting of rats undergoing hemorrhagic shock and patients with inflammatory bowel disease (IBD) (paragraph 2.4). 


\subsection{A pilot study on the non- invasive evaluation of intestinal damage in celiac disease using I-FABP and L-FABP}

Joep P. M. Derikx, Anita C. E. Vreugdenhil, Anita M. Van den Neucker, Joep Grootjans, Annemarie A. van Bijnen, Jan G. M. C. Damoiseaux, L. W. Ernest van Heurn, Erik Heineman, Wim A. Buurman Journal of Clinical Gastroenterology, in press. 


\begin{abstract}

\section{Background and goals}

In the clinical management of celiac disease, new non-invasive tools for evaluation of intestinal damage are needed for diagnosis and for follow-up of diet effects. Fatty acid binding proteins (FABP) are potentially useful for this purpose since these are small cytosolic proteins present in enterocytes and sensitive markers for intestinal mucosal damage. First, the distribution and microscopic localization of FABP in the healthy human intestine was examined. Second, levels of circulating FABP were measured in patients with celiac disease before and after introducing a gluten-free diet (GFD) and in healthy controls.
\end{abstract}

\title{
Study
}

The distribution and microscopic localization of FABP in normal human intestinal tissue was assessed using surgical intestinal specimens of 39 patients. Circulating levels of Intestinal (I)-FABP and Liver (L)-FABP were determined in 26 healthy volunteers and 13 patients with biopsy proven celiac disease. Ten of these patients were re-evaluated within one year after starting GFD.

\section{Results}

I- and L-FABP are predominantly present in the small intestine, mainly the jejunum. Moreover, FABP are expressed in cells on the upper part of the villi, the initial site of destruction in celiac disease. Circulating levels of FABP are significantly elevated in untreated patients with biopsy proven celiac disease compared to healthy controls (I-FABP: $784.7 \mathrm{pg} / \mathrm{ml}$ vs. $172.7 \mathrm{pg} / \mathrm{ml}, \mathrm{p}<0.001$; L-FABP: $48.4 \mathrm{ng} / \mathrm{ml}$ vs. $10.4 \mathrm{ng} / \mathrm{ml}, \mathrm{p}<0.001)$. In response to GFD these concentrations normalize.

\section{Conclusions}

Results of this pilot study strongly suggest that FABP can be used as a noninvasive method for assessment of intestinal damage in celiac disease. Besides an additional role in the diagnosis of celiac disease, FABP potentially enable non-invasive monitoring of the GFD effects. 


\section{INTRODUCTION}

Celiac disease is an immune-mediated disorder characterized by atrophy of intestinal villi, with a relatively high prevalence. The mucosal destruction has serious consequences including malabsorption, malnutrition and failure to thrive in young children. In the long term refractory sprue and enteropathyassociated T-cell lymphoma may develop despite a strict gluten-free diet (GFD).$^{1-3} \mathrm{~A}$ major concern in the clinical management of celiac disease is the necessity of mucosal biopsies of the proximal small bowel for evaluation of intestinal damage and confirmation of the diagnosis. ${ }^{1-3}$ In children, mucosal biopsies are taken using gastroduodenoscopy under general anaesthesia. This is an invasive and expensive procedure with the risk of complications and a substantial emotional impact on the child and its parents/caretakers. Besides these diagnostic concerns, the follow-up of celiac disease is hampered by the absence of a sensitive non-invasive test for evaluation of the effects of a GFD on the recovery of intestinal epithelial cells. Serum anti-endomysial antibodies (IgA-EMA) and anti-tissue transglutaminase (IgA-tTG) antibody levels are well established markers for screening in older children and adults, but are due to their long half-life not accurate enough for evaluation of the diet effect in celiac disease. ${ }^{4,5}$ Moreover, seronegative celiac disease is frequently present in patients with only minor pathological changes in the intestinal mucosa and in older patients with more severe disease ${ }^{6-8}$ Furthermore, the reliability of the serological tests for either monitoring compliance or histological response to treatment is still under discussion. ${ }^{9-12}$

New diagnostic markers for intestinal damage are needed to enable noninvasive diagnosis and rapid evaluation of the effects of GFD and gluten challenge.

Previous studies have identified fatty acid binding proteins (FABP) as circulating and urinary markers for intestinal epithelial cell damage in mesenterial thrombosis and necrotising enterocolitis. ${ }^{13,14}$ FABP are cytosolic proteins present in enterocytes which are rapidly released into the systemic circulation upon cell damage since these proteins are unbound and very small $(15 \mathrm{kDa})$. Three FABP subtypes are present in the gut; intestinal fatty acid binding protein (I-FABP), liver fatty acid binding protein (L-FABP) and ileal bile acid binding protein (I-BABP). ${ }^{15}$ Pelsers et al. reported that I-FABP and L-FABP are present in the small and large bowel with predominance in the jejunum. ${ }^{16}$ The first objective of this pilot study was to assess the distribution and the microscopic localization of FABP in the human gastrointestinal tract. Second, the potential usefulness of FABP as markers for intestinal epithelial damage in 


\section{Chapter 2}

celiac disease was examined. Circulating FABP levels in celiac disease were compared with levels in healthy volunteers. Third, we studied whether FABP levels normalize after starting a GFD and therefore if assessment of circulating levels of FABP are possibly of value in the follow-up of celiac disease.

\section{MATERIALS AND METHODS}

\section{Distribution and microscopic localization of FABP in normal human intestine}

To study the distribution and microscopic localization of I-FABP and L-FABP throughout the gastrointestinal tract, histological normal human intestinal full thickness tissue was obtained during routine surgery (such as closure of stoma) of 39 patients (18F:21M; mean age: 6.5 years, range: 5 days-60 years) at the University Hospital Maastricht after appropriate informed consent. Stomach $(n=2)$, duodenum $(n=3)$, jejunum $(n=7)$, ileum $(n=22)$, caecum $(n=2)$, appendix $(n=3)$, ascending colon $(n=9)$, transversal colon $(n=2)$, descending colon $(n=2)$ and sigmoid colon $(n=12)$ were collected. The tissue samples were directly frozen in liquid nitrogen or fixed in formalin and embedded in paraffin. The study was approved by the local medical ethical committee.

Expression and quantification of FABP from stomach to sigmoid colon was performed by western blot analysis and enzyme-linked immunosorbent assay (ELISA).

Before analyses, intestinal tissue samples were homogenized in lysis buffer $(200 \mathrm{mM} \mathrm{NaCl}, 10 \mathrm{mM}$ Tris base, $5 \mathrm{mM}$ EDTA, 10\% Glycerin, $1 \mathrm{mM}$ PMSF, $0.1 \mathrm{U} / \mathrm{ml}$ Aprotinin and $1 \mu \mathrm{g} / \mathrm{ml}$ Leupeptin) using an Ultra-Turrax homogenizer (IKA Werke, Breisgau, Germany). Homogenates were centrifuged at $1000 \mathrm{~g}$, and supernatants, containing the cytoplasmic proteins, were collected.

First, expression of FABP was semi-quantified by western blotting. Total protein concentrations were determined using the Bradford method. Aliquots with equal amounts of protein were heated at $100^{\circ} \mathrm{C}$ for $5 \mathrm{~min}$ in SDS sample buffer, separated on 15\% SDS-polyacrylamide gels and transferred to polyvinylidene fluoride membrane (Immobilin P, Millipore, Bedford, MA). After transfer of proteins, a blocking step, antibody incubation and washing of the membrane, were performed in PBS with 3\% non-fat dry milk and $0.05 \%$ Tween. Anti human I-FABP and L-FABP antibodies were kindly provided by Hycult Biotechnology (HBT, Uden, the Netherlands). Membranes were incubated with the appropriate horseradish peroxidase-conjugated secondary antibodies (DAKO, Denmark). Positive bands were visualized using chemiluminescence (Supersignal, Pierce, Rockford, IL). 
Second, concentrations of tissue I-FABP and L-FABP per gram wet tissue were determined using highly specific commercially available ELISA that selectively detects human I-FABP (standard: 20-5,000 pg/ml) and L-FABP (standard: 0.1$25.0 \mathrm{ng} / \mathrm{ml}$ ), provided by HBT.

In addition, the microscopic localization of FABP in the intestine was performed with immunohistochemistry on paraffin embedded sections. Sections cut at $4 \mu \mathrm{m}$ were deparaffinized and pre-treated with $0.3 \%$ hydrogen peroxide in methanol for 15 min to block endogenous peroxidase activity. After blocking the non-specific antibody binding using 5\% BSA, the sections were incubated with specific antibodies to human I-FABP and L-FABP (both kindly provided by $\mathrm{HBT}$ ) at room temperature for $45 \mathrm{~min}$. After washing, an appropriate biotinconjugated secondary antibody was used. Binding of the primary antibody was demonstrated by the streptavidin-biotin system (DAKO, Denmark) and visualized by applying 3-amino-9-ethylcarbazole (AEC; Sigma, St. Louis, MO). Nuclei were counterstained with haematoxylin. The stained slides were photographed by a Nikon eclipse E800 microscope with a Nikon digital camera DXM1200F. No significant staining was detected in slides incubated with control rabbit serum and/or mouse IgG instead of the primary antibody indicating the absence of significant background staining.

\section{Patients with celiac disease and healthy volunteers}

Thirteen patients with newly diagnosed celiac disease who visited the University Hospital Maastricht between 2000 and 2004 were retrospectively studied. We included all patients of whom rest-serum, which was stored at $-80^{\circ} \mathrm{C}$ until analysis, was collected maximally three months before celiac disease was diagnosed. The diagnosis was confirmed by histological establishment of duodenal villous atrophy. None of the included patients was IgA deficient. In 10 of 13 patients a second serum sample was collected within one year after starting the GFD. From the hospital charts, the following data were recorded: age, sex, gastrointestinal signs and symptoms, serum levels of anti-endomysial (Scimedx, Denville, New Jersey) and anti-tissue transglutaminase IgA antibodies (Phadia GmbH, Freiburg, Germany) (routinely measured by the laboratory of clinical immunology in the University Hospital Maastricht) and histological findings of the small intestinal biopsy.

Twenty-six healthy volunteers $(\mathrm{M}: \mathrm{F}=17: 9)$ were recruited to establish normal values for serum I-FABP and L-FABP. The mean age in this control group was 22 years (range: $1-61$ years).

Serum I-FABP and L-FABP concentrations were determined using previously described ELISAs that detect human I-FABP and L-FABP, kindly provided by HBT. The local ethics committee approved the study. 


\section{Chapter 2}

\section{Statistical analyses}

Statistical analyses were performed with Prism 4.0 for Windows (GraphPad Software Inc. San Diego, CA). Intestinal and serum FABP concentrations were presented as mean \pm standard error (SEM). Normality of all data obtained was verified by Kolmogorov-Smirnov test.

Concentrations of serum FABP were compared between patients with celiac disease and healthy individuals using unpaired t-test. Levels of serum FABP of untreated patients were compared with levels after introduction of a GFD using paired t-test.

In order to find the cut-off point of FABP serum levels that most accurately discriminate patients with celiac disease from healthy individuals, a receiver operating characteristic (ROC) curve was drawn by plotting sensitivity against 1 -specificity for all possible serum FABP thresholds. The overall accuracy of serum FABP in detecting celiac disease was summarized using the area under the curve $(A \cup C)$, with an $A \cup C$ of 0.5 indicating no discrimination ability. Ninety-five percent confidence intervals $(\mathrm{Cl})$ for the sensitivity, specificity, positive and negative likelihood ratios (LR) were calculated for the best cut-off values, defined as the maximal sum of sensitivity and specificity. A p-value below 0.05 was considered statistically significant.

\section{ResULtS}

\section{Distribution of I-FABP and L-FABP in normal human intestine}

The concentration of I-FABP varies along the small intestine, and is low in the complete colon (results from ELISA are shown in Figure 1a; results from western blot analysis are not shown). Maximal I-FABP expression is found in the jejunum $(22.5( \pm 7.3) \mu \mathrm{g}$ I-FABP/g tissue) with lower expression in the ileum and duodenum $(4.8( \pm 1.6) \mu \mathrm{g} \mathrm{I}-\mathrm{FABP} / \mathrm{g}$ tissue and $4.6( \pm 0.6) \mu \mathrm{g} \mathrm{I-FABP} / \mathrm{g}$ tissue, respectively). Expression throughout the whole colon is minimal $(0.5( \pm 0.1) \mathrm{mg}$ I-FABP/g tissue).

The distribution pattern of L-FABP in the healthy human gastrointestinal tract is comparable with that of I-FABP (Figure 1b). The jejunum contains the highest concentration of L-FABP (829.6 ( \pm 96.1$) \mu \mathrm{g}$ L-FABP/g tissue), whereas the rest of the small intestine (ileum: $105.3( \pm 25.0) \mu \mathrm{g} \mathrm{L-FABP/g}$ tissue) and colon (102.3 $( \pm 15.4) \mu \mathrm{g}$ L-FABP/g tissue) contain low concentrations. 

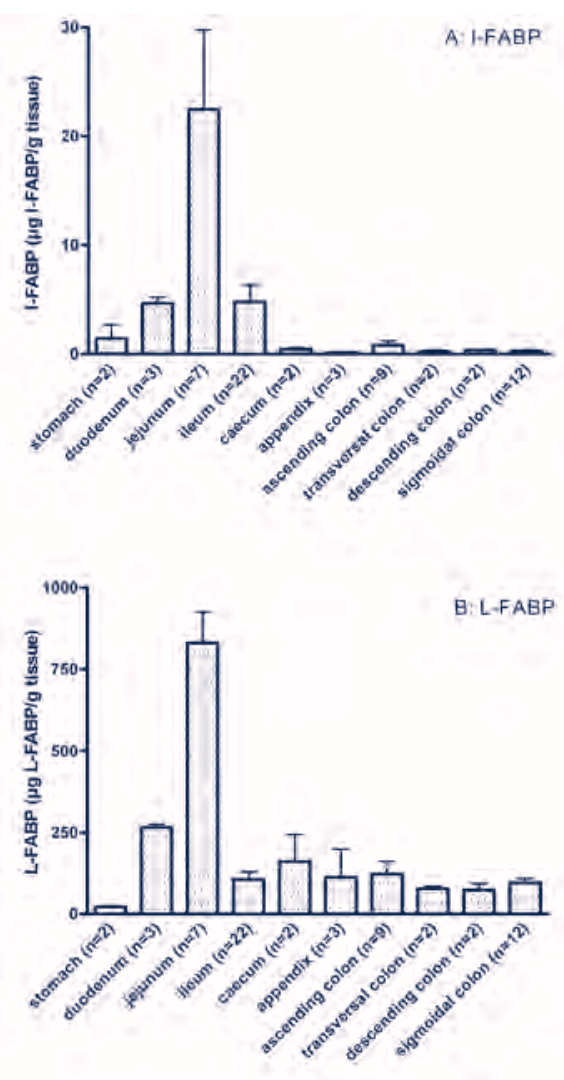

Figure 1: Distribution of I-FABP (a) and L-FABP (b) in histologically normal human intestine. Data are mean values with SEM from 39 patients.

\section{Microscopic localization of FABP in normal human intestine}

Intensive immunostaining for I-FABP and L-FABP was observed in the healthy ileal and jejunal epithelium, mainly in the absorptive cells. Figure 2 is a representative image for the ileum and jejunum, showing the presence of both I-FABP and L-FABP in the cytoplasm of the mature enterocytes and goblet cells in the upper half of the villi, whereas cells in the crypts were not immunopositive.

The healthy colon showed immunoreactive cytoplasm of the epithelial cells in the upper half of the crypts for I-FABP and L-FABP (data not shown). FABP were not detected in cells in the bottom of the crypts. 


\section{Chapter 2}
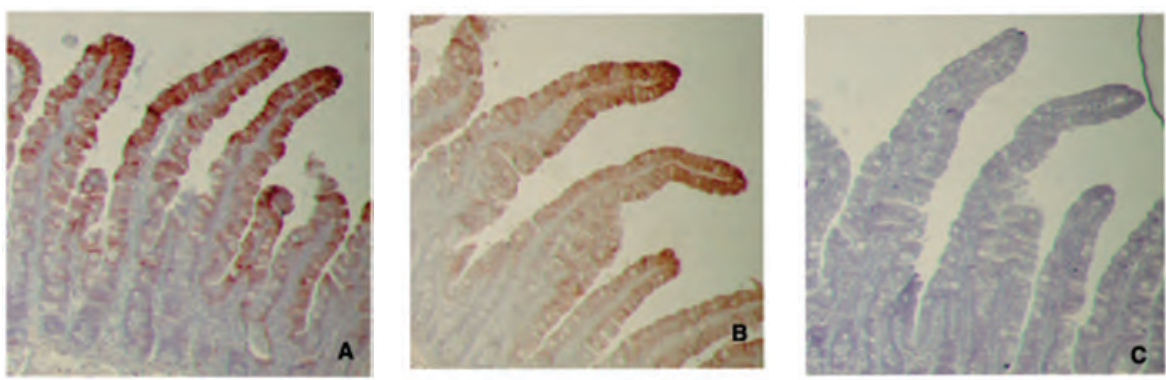

Figure 2: Immunolocalization of L-FABP (a) and I-FABP (b) at a 100x magnification in normal jejunum. In image (c), the primary antibody is replaced by control serum. I-FABP and L-FABP are immunostained in the upper half of the villus.

\section{Baseline characteristics of patients with celiac disease and healthy individuals}

Celiac disease was confirmed in 13 patients by biopsy of the small intestine; 3 patients had Marsh-stage 2 and 10 patients had Marsh-stage 3 (Table 1). The mean age was 30 years (range: 1-70 years). In 11 patients anti-endomysial antibodies (IgA-EMA) were present, while in 1 patient (patient 5) IgA-EMA was not detected and in 1 patient (patient 3) IgA-EMA level was borderline positive. Antibodies directed against tissue transglutaminase (IgA-tTG) were detected in 12 patients, while in 1 patient (patient 3) IgA-tTG was absent. Of the whole group, 1 patient did not clinically respond to a GFD (patient 13); gastrointestinal symptoms persisted and repeated duodenal biopsies showed inflammation in the intestine and atrophy of the intestinal villi. Despite this disease activity, IgA-tTG became negative and IgA-EMA showed borderline values within one year of GFD. In all other patients symptoms disappeared after elimination of gluten from the diet. However, IgA-tTG and/or IgA-EMA remained positive in 5 patients. 
Table 1: baseline characteristics of 13 patients with celiac disease and their serologies and circulating FABP values at the moment of diagnosis and within 1 year after the start of the glutenfree diet (GFD). IgA-tTG: $>10 \mathrm{U} / \mathrm{ml}=$ positive, 7-10 U/ml=borderline, $<7 \mathrm{U} / \mathrm{ml}=$ =negative. IgA-EMA: titre 1:10 (starting dilution) $=$ positive.

IgA-tTG: anti-tissue transglutaminase antibodies; IgA-EMA: anti-endomysial antibodies; neg=negative; pos=positive; I-FABP: intestinal-fatty acid binding protein; L-FABP: liver-fatty acid binding protein

* Patient 13 is the only patient with persisting gastrointestinal complaints after introduction of GFD. Repeated duodenal biopsies of this patient showed an inflammatory response in the small intestine, possibly explained by a refractory sprue syndrome.

\begin{tabular}{|c|c|c|c|c|c|c|c|c|c|c|}
\hline Patient & $\begin{array}{c}\text { Age at } \\
\text { diagnosis } \\
\text { (years) }\end{array}$ & $\begin{array}{l}\text { Marsh- } \\
\text { classifi- } \\
\text { cation }\end{array}$ & $\begin{array}{c}\text { IgA-tTG at } \\
\text { diagnosis } \\
(\mathrm{U} / \mathrm{ml})\end{array}$ & $\begin{array}{c}\text { Iga-EMA at } \\
\text { diagnosis } \\
\text { (titre) }\end{array}$ & $\begin{array}{c}\text { I-FABP at } \\
\text { diagnosis } \\
(\mathrm{pg} / \mathrm{m})\end{array}$ & $\begin{array}{c}\text { L-FABP at } \\
\text { diagnosis } \\
(\mathrm{ng} / \mathrm{ml})\end{array}$ & $\begin{array}{c}\text { IgA-TtG } \\
\text { within } 1 \\
\text { year GFD } \\
(\mathrm{U} / \mathrm{ml})\end{array}$ & $\begin{array}{c}\text { IgA-EMA } \\
\text { within } 1 \\
\text { year GFD } \\
\text { (titre) }\end{array}$ & $\begin{array}{c}\text { I-FABP } \\
\text { within } 1 \\
\text { year (pg/m) }\end{array}$ & $\begin{array}{c}\text { L-FABP } \\
\text { within } 1 \\
\text { year } \\
\text { (ng/ml) }\end{array}$ \\
\hline 1 & 65 & 3 & 120 & pos & 766 & 65.8 & - & - & - & \\
\hline 2 & 1 & 3 & 462 & pos & 1136 & 93.7 & 1 & neg & 206 & 7.3 \\
\hline 3 & 70 & 3 & 1 & borderline & 459 & 26.4 & 1 & neg & 260 & 16.8 \\
\hline 4 & 62 & 2 & 92 & pos & 695 & 35.7 & 25 & pos & 403 & 15.3 \\
\hline 5 & 62 & 2 & 12 & neg & 445 & 19.8 & 8 & pos & 359 & 9.6 \\
\hline 6 & 1 & 3 & $>5000$ & pos & 1944 & 146.1 & - & & & \\
\hline 7 & 9 & 2 & 81 & pos & 241 & 9.3 & - & - & - & \\
\hline 8 & 4 & 3 & 155 & pos & 451 & 23.6 & 1 & pos & 41 & 19.0 \\
\hline 10 & & 83 & 108 & pos & 587 & 6.9 & 13 & pos & 57 & 8.6 \\
\hline 11 & 51 & 3 & 255 & pos & 1277 & 93.2 & 7 & pos & 192 & 11.7 \\
\hline $13^{*}$ & 47 & 3 & 27 & pos & 1386 & 63.0 & 1 & borderline & 643 & 34.3 \\
\hline
\end{tabular}

\section{Serum I-FABP and L-FABP levels in patients with celiac disease}

The mean serum I-FABP concentration in normal healthy individuals was $172.7 \pm 20.2 \mathrm{pg} / \mathrm{ml}$. The mean serum I-FABP concentration in patients with celiac disease $(784.7 \pm 145.5 \mathrm{pg} / \mathrm{ml})$ was 4.5 times higher than in serum of normal individuals $(p<0.001$, Figure $3 a)$. Also serum L-FABP levels were significantly higher in patients with celiac disease compared with healthy volunteers $(48.4 \pm 11.6 \mathrm{ng} / \mathrm{ml}$ vs. $10.4 \pm 0.7 \mathrm{ng} / \mathrm{ml}, \mathrm{p}<0.001$, Figure $3 \mathrm{~b}$ ).

The ability of serum I-FABP and L-FABP concentrations to discriminate between patients with celiac disease and healthy individuals was analyzed by ROC curves (Figures 4a, b). The ROC curve for serum I-FABP yielded a high AUC (0.90 (95\% Cl: 0.76-1.05) p<0.001) with an optimal cut-off value of 405.1 


\section{Chapter 2}

$\mathrm{pg} / \mathrm{ml}$. The ROC curve of L-FABP had an AUC of 0.86 (95\% Cl: 0.70-1.02, $\mathrm{p}<0.001)$ and the best threshold level of serum L-FABP in separating patients with celiac disease from healthy individuals was $18.1 \mathrm{ng} / \mathrm{ml}$
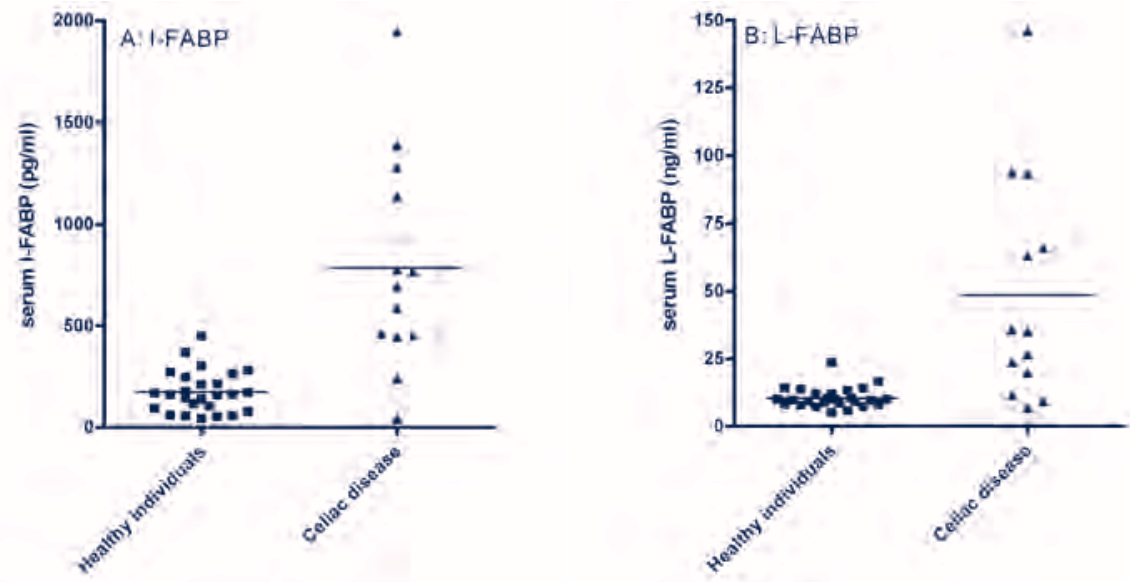

Figure 3: Elevated concentrations of serum I-FABP (a) and L-FABP (b) in patients with celiac disease compared to healthy individuals. The line represents the mean value of serum FABP levels.
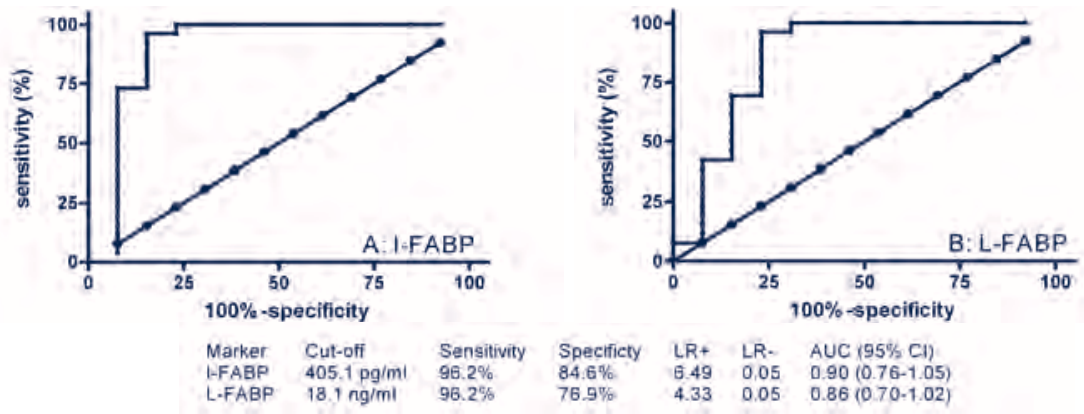

Figure 4: Serum levels of I-FABP (a) and L-FABP (b) discriminate between patients with celiac disease and healthy individuals. The ability of serum FABP levels to discriminate was assessed by ROC curves (sensitivity vs. 100\%-specificity), obtained by varying the cut-off values of concentrations.

Serum I-FABP and L-FABP values were measured in 10 patients within 1 year after starting the GFD (Figure 5). These patients had a significant reduction in IFABP from a mean level of $725.5 \pm 134.4 \mathrm{pg} / \mathrm{ml}$ before the introduction of GFD to $266.8 \mathrm{pg} / \mathrm{ml}$ (range: $41.0-642.6 \mathrm{pg} / \mathrm{ml}$ ) within 1 year of GFD $(p=0.002)$. 
Mean serum L-FABP concentration decreased significantly from $40.9 \pm 10.0$ $\mathrm{ng} / \mathrm{ml}$ at the moment of diagnosis to $15.3 \mathrm{ng} / \mathrm{ml}$ (range: $7.3-34.3 \mathrm{ng} / \mathrm{ml}$ ) in patients on GFD ( $p=0.025)$. Nine of 10 patients tested within 1 year of GFD obtained I-FABP serum values below the cut-off value of $405.1 \mathrm{pg} / \mathrm{ml}$. In 1 patient I-FABP levels remained enhanced (patient 13, Table 1). In this patient gastrointestinal symptoms persisted and repeated duodenal biopsies showed inflammation and atrophy of villi. For L-FABP similar data were obtained. Eight of 10 patients tested within 1 year of GFD had L-FABP serum values below the threshold value of $18.1 \mathrm{ng} / \mathrm{ml}$. One of the patients with elevated circulating values of L-FABP within 1 year of GFD (patient 13, Table 1) had continuing abdominal symptoms and villus damage on biopsies.
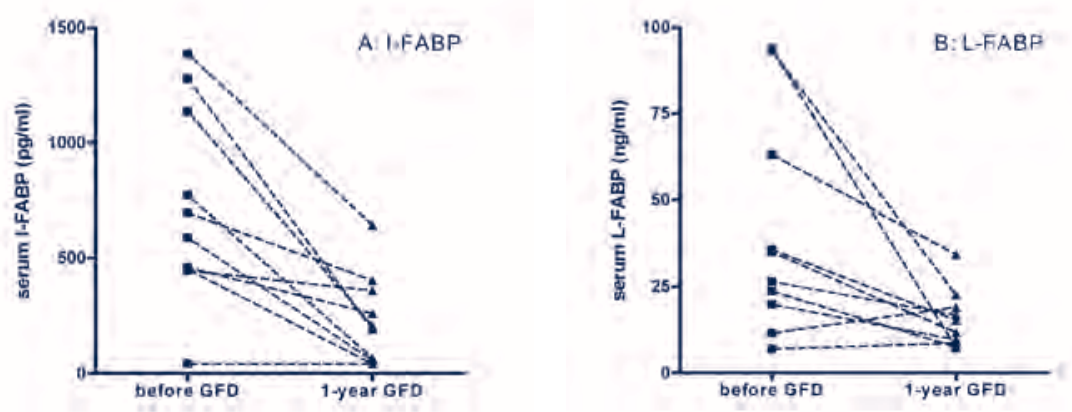

Figure 5: Serum I-FABP (a) and L-FABP (b) concentration before and after starting a gluten-free diet (GFD). 


\section{Chapter 2}

\section{Discussion}

The value of the biomarkers I-FABP and L-FABP in the evaluation of intestinal damage and recovery of the intestinal mucosa in celiac disease was studied. Currently, duodenal biopsy is required for establishing mucosal damage and villous atrophy. A non-invasive diagnostic method for confirmation of the diagnosis celiac disease is urgently needed in addition to the serological tests. Besides new diagnostic tools for celiac disease, there is a strong need for reliable markers detecting recovery of the intestinal mucosa in response to a GFD, since the currently used serologic tests have poor correlation with mucosal damage. Therefore, in this study serum FABP concentrations as markers for epithelial cell damage were measured in patients with celiac disease before and after introduction of the GFD.

First, the expression of I-FABP and L-FABP in the different segments of the human intestine was evaluated. I-FABP and L-FABP are expressed in the mucosa of the entire small intestine and have a predominant expression in the jejunum. I- FABP and L-FABP are in particular highly expressed in cells present on the tops of the villi. Since villous atrophy in the small intestine is the hallmark of celiac disease ${ }^{1}$, we consider circulating levels of FABP to be potential markers of disease activity in celiac disease. The presence of FABP on the tops of the villi, the initial site of destruction in celiac disease, makes circulating I-FABP and L-FABP also potentially useful in an early stage of celiac disease.

The data of the present pilot-study show that I- and L-FABP serum levels are significantly increased in untreated celiac disease compared to healthy controls and are sensitive markers for intestinal damage in celiac disease. However, in one untreated patient the I-FABP and L-FABP concentration was below the cut-off value as determined in this study (patient 9). Histological evaluation of the biopsy of this patient showed a Marsh 3b classification, representing a substantial loss of the absolute enterocyte mass and especially the villous enterocytes; thus, a loss of cells containing FABP. It is conceivable that, because of extensive denudation, the amount of 'shedding' cells that contain FABP is low, resulting in low circulating FABP values.

In one patient with Marsh 3 (patient 3) IgA-tTG was not elevated and IgAEMA showed a borderline result. However, I-FABP and L-FABP were both above cut-off values. These findings further emphasize that FABP measurements can contribute to screening for celiac disease.

In all but one patient in this study the I- and L-FABP concentration normalized after implementation of the GFD. In this single patient (patient 13) 
gastrointestinal complaints also persisted and repeated duodenal biopsies of this patient showed an inflammatory response in the small intestine, possibly explained by a refractory sprue syndrome. IgA-tTG levels became negative in this patient after introduction of the GFD and IgA-EMA titres decreased to borderline levels. This discrepancy between serologic titres and recovery of the intestinal damage after start of GFD was also found in earlier studies. ${ }^{9-12}$ The finding of persistent elevated FABP concentrations in this patient further signifies the potential usefulness of circulating levels of FABP as markers to follow-up celiac disease activity. All the other patients improved clinically on GFD, which was consistent with FABP recovery; repeat biopsies were not performed.

The results of this study suggest that assessment of circulating FABP concentrations seem very valuable to monitor the effect of GFD on recovery of the intestinal mucosa in celiac disease. This is in line with earlier observations from animal studies showing that serum levels of I-FABP correlate with the phases of histological mucosal injury after intestinal ischemia-reperfusion. ${ }^{15,17,18}$ This finding is of major clinical importance since there are no non-invasive tools to evaluate diet effects and therapy compliance. IgA-EMA and IgA-tTG, frequently used for this purpose in clinical practice, were shown to be poor predictors of dietary transgression. ${ }^{9-12}$

In contrast to I-FABP, L-FABP shows a multi-tissue expression. Besides the expression in the intestine, L-FABP is also present in hepatocytes and tubular cells of the kidney. ${ }^{15}$ Increased L-FABP levels in serum can therefore be derived from other organs then the intestine. I-FABP is exclusively expressed in the intestine and the test accuracy of I-FABP is higher than for L-FABP. Therefore IFABP measurement is preferable for diagnosis and follow-up in celiac disease.

Prior studies demonstrated that the renal clearance of FABP is very fast; the half-life of L-FABP was approximately eleven minutes. ${ }^{19}$ From these data it can be hypothesized that urinary FABP levels correlate with plasma levels and disease activity. One of our future goals is to evaluate the correlation of urinary FABP levels with intestinal damage in celiac disease.

Based on the data of this pilot study, we speculate that in children with both a strong clinical suspicion of celiac disease and positive serological tests, establishment of a pattern of high circulating I-FABP levels at diagnosis, followed by declined levels after gluten elimination and increased levels after gluten challenge might contribute to the clinical management of celiac disease. Further studies are necessary to determine whether assessment of intestinal epithelial cell damage by FABP analysis can replace the invasive procedure of endoscopy and mucosa biopsy. In particular for children the drawing of blood or even the mere sampling of urine will be an important advantage above 


\section{Chapter 2}

endoscopy. Besides a possible additional role for FABP in the diagnosis of celiac disease, assessment of urinary concentration of I-FABP and L-FABP may provide a new important non-invasive tool to monitor the short-term response to a GFD and in this way contribute importantly to the follow-up of celiac patients. A prospective study is now performed to further evaluate the value of FABP in the clinical management of celiac disease.

\section{REFERENCES}

1 Chand N, Mihas AA. Celiac disease: current concepts in diagnosis and treatment. J Clin Gastroenterol. 2006;40:3-14.

2 Green PH, Cellier C. Celiac disease. N Engl J Med. 2007;357:1731-1743.

3 Hopper AD, Hadjivassiliou M, Butt S, et al. Adult coeliac disease. Bmj. 2007;335:558562.

4 Rostom A, Dube C, Cranney A, et al. The diagnostic accuracy of serologic tests for celiac disease: a systematic review. Gastroenterology. 2005;128:S38-46.

5 Pietzak MM. Follow-up of patients with celiac disease: achieving compliance with treatment. Gastroenterology. 2005;128:S135-141.

6 Salmi TT, Collin P, Korponay-Szabo IR, et al. Endomysial antibody-negative coeliac disease: clinical characteristics and intestinal autoantibody deposits. Gut. 2006;55:1746-1753.

7 Esteve M, Rosinach M, Fernandez-Banares F, et al. Spectrum of gluten-sensitive enteropathy in first-degree relatives of patients with coeliac disease: clinical relevance of lymphocytic enteritis. Gut. 2006;55:1739-1745.

8 Sanders DS, Hurlstone DP, McAlindon ME, et al. Antibody negative coeliac disease presenting in elderly people--an easily missed diagnosis. Bmj. 2005;330:775-776.

9 Vahedi K, Mascart F, Mary JY, et al. Reliability of antitransglutaminase antibodies as predictors of gluten-free diet compliance in adult celiac disease. Am / Gastroenterol. 2003;98:1079-1087.

10 Kaukinen K, Sulkanen S, Maki M, et al. IgA-class transglutaminase antibodies in evaluating the efficacy of gluten-free diet in coeliac disease. Eur J Gastroenterol Hepatol. 2002;14:311-315.

11 Dickey W, Hughes DF, McMillan SA. Disappearance of endomysial antibodies in treated celiac disease does not indicate histological recovery. Am J Gastroenterol. 2000;95:712-714.

12 Tursi A, Brandimarte G, Giorgetti GM. Lack of usefulness of anti-transglutaminase antibodies in assessing histologic recovery after gluten-free diet in celiac disease. J Clin Gastroenterol. 2003;37:387-391. 
13 Kanda T, Fujii H, Tani T, et al. Intestinal fatty acid-binding protein is a useful diagnostic marker for mesenteric infarction in humans. Gastroenterology. 1996;110:339-343.

14 Derikx JP, Evennett NJ, Degraeuwe PL, et al. Urine based detection of intestinal mucosal cell damage in neonates with suspected necrotising enterocolitis. Gut. 2007; 56:1473-1475.

15 Pelsers MM, Hermens WT, Glatz JF. Fatty acid-binding proteins as plasma markers of tissue injury. Clin Chim Acta. 2005;352:15-35.

16 Pelsers MM, Namiot Z, Kisielewski W, et al. Intestinal-type and liver-type fatty acidbinding protein in the intestine. Tissue distribution and clinical utility. Clin Biochem. 2003;36:529-535.

17 Gollin G, Marks, C, Marks WH. Intestinal fatty acid binding protein in serum and urine reflects early ischemic injury to the small bowel. Surgery. 1992;113:545-551.

18 Kanda T, Nakatomi Y, Ishikawa $\mathrm{H}$, et al. Intestinal fatty acid-binding protein was a sensitive marker for intestinal ischemia. Dig Dis Sci. 1992;37:1362-1367.

19 van de Poll MC, Derikx JP, Buurman WA, et al. Liver manipulation causes hepatocyte injury and precedes systemic inflammation in patients undergoing liver resection. World J Surg. 2007;31:2033-2038. 


\subsection{Liver manipulation causes hepatocyte injury and precedes systemic inflammation in patients undergoing liver resection}

Marcel C. G. van de Poll *, Joep P. M. Derikx *, Wim A. Buurman, Wilbert H. M. Peters, Hennie M. J. Roelofs, Stephen J. Wigmore, Cornelis H. C. Dejong. * both authors contributed equally to this manuscript World Journal of Surgery (2007) 31: 2033-8. 


\begin{abstract}

\section{Background}

Liver failure following liver surgery is caused by an insufficient functioning remnant cell mass. This can be due to insufficient liver volume and can be aggravated by additional cell death during or after surgery. The aim of this study was to elucidate the causes of hepatocellular injury in patients undergoing liver resection.
\end{abstract}

\title{
Methods
}

Markers of hepatocyte injury (AST, GSTa, and L-FABP) and inflammation (IL-6) were measured in plasma of patients undergoing liver resection with and without intermittent inflow occlusion. To study the separate involvement of the intestines and the liver in systemic LFABP release, arteriovenous concentration differences for L-FABP were measured.

\section{Results}

During liver manipulation, liver injury markers increased significantly. Arterial plasma levels and transhepatic and transintestinal concentration gradients of LFABP indicated that this increase was exclusively due to hepatic and not due to intestinal release. Intermittent hepatic inflow occlusion, anesthesia, and liver transection did not further enhance arterial L-FABP and GSTa levels. Hepatocyte injury was followed by an inflammatory response.

\section{Conclusions}

This study shows that liver manipulation is a leading cause of hepatocyte injury during liver surgery. A potential causal relation between liver manipulation and systemic inflammation remains to be established; but since the inflammatory response is apparently initiated early during major abdominal surgery, interventions aimed at reducing postoperative inflammation and related complications should be started early during surgery or beforehand. 


\section{INTRODUCTION}

Liver failure is a severe complication of liver surgery, occurring when there is insufficient remnant cell mass postoperatively. This can be due to excessive resection, leaving a too small remnant liver volume, but in some cases liver failure occurs in patients with a seemingly sufficient remnant liver volume ${ }^{1}$. In these cases the functional capacity of the remaining cell mass is probably impaired by secondary processes. Recognition and modulation of factors impairing cell survival is crucial to enhance liver function following liver surgery. In this context much attention has been paid to the effects of ischemia and reperfusion.

Ischemia-reperfusion is a frequently encountered phenomenon in liver resection, caused by temporary occlusion of blood flow to the liver (Pringle maneuver), which is a popular way to reduce blood loss during parenchymal liver transection. Ischemia-reperfusion induces energy depletion and generation of reactive oxygen species. Although several endogenous protection mechanisms aimed at supporting cell survival are activated during ischemiareperfusion ${ }^{2}$, excessive energy depletion or oxidative stress ultimately results in apoptotic or necrotic cell death.

Interestingly, it has been shown that plasma levels of markers for hepatocellular injury increase before liver transection and before application of the Pringle maneuver, suggesting that factors other than ischemia-reperfusion may cause hepatocyte demise during liver surgery ${ }^{3}$. In this context a role has been proposed for the hepatotoxic effects of anesthesia ${ }^{4}$, the effects of systemic inflammation, probably secondary to intestinal manipulation ${ }^{5}$, and the effects of manipulation of the liver itself during perihepatic dissection and mobilization ${ }^{6}$. The aim of this study was to elucidate the causes of early hepatocellular injury in patients undergoing liver resection.

\section{Methods}

\section{Patients}

Patients undergoing liver resection for secondary tumors in an otherwise healthy liver were studied (Table 1). All patients were anesthetized according to institutional routines using isoflurane and propofol. Surgery was commenced using a subcostal bilateral incision. Olivier retractors (Copharm, Abcoude, Holland) or Omni-Tracts were used to improve exposure. Before parenchymal division the liver was mobilized as described elsewhere, followed by intraoperative ultrasound ${ }^{7}$. Cholecystectomy was performed routinely before 
Table 1: Patient characteristics

\begin{tabular}{lcc} 
& Hepatectomy w/o IPM $(\mathrm{n}=11)$ & Hepatectomy with IPM $(\mathrm{n}=9)$ \\
\hline Age (years) & $57(36-72)$ & $54(33-75)$ \\
Male: Female & $9: 2$ & $5: 4$ \\
AST (IU/L) & $26(13-40)$ & $23(7-37)$ \\
LDH (IU/L) & $337(1-558)$ & $377(295-490)$ \\
Albumin (g/L) & $39.0(33.9-43.9)$ & $37.6(33.7-43.9)$ \\
CRP (mg/L) & $3.5(<1.0-8.7)$ & $5.0(<1.0-8.7)$ \\
Bilirubin ( $\mu$ mol/L) & $13.4(7.7-18.7)$ & $12.9(8.4-21)$ \\
\hline IPM = intermittent Pringle maneuver & \\
Blood chemistry was obtained during routine assessment 1 day preoperatively. Values are \\
expressed as median (range)
\end{tabular}

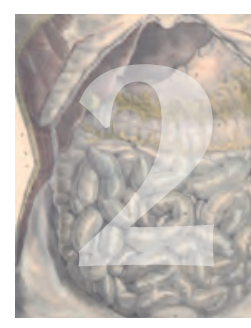

liver transection. All patients had indwelling radial artery catheters placed for continuous monitoring of arterial blood pressure and blood sampling.

Patients were nonrandomly assigned to one of two protocols (described below). Assignment was based upon the surgeon's preference of using the Pringle maneuver or not. If applied, an intermittent Pringle maneuver (15 min of ischemia, $5 \mathrm{~min}$ of reperfusion) was used by rubberband ligation of the hepatoduodenal ligament.

\section{Effects of intermittent Pringle maneuver on hepatocellular injury}

From nine patients undergoing liver resection with intermittent Pringle maneuver, arterial blood was sampled preoperatively before and after each period of $15 \mathrm{~min}$ of ischemia and $5 \mathrm{~min}$ of reperfusion and 90 minutes postoperatively. Systemic concentrations of two markers of hepatocellular injury, aspartate amino transferase (AST) and glutathione-s-transferase-a (GSTa), were measured as well as plasma levels of liver-type fatty acid binding protein (L-FABP). Methods of blood processing and laboratory analyses are described below. In addition, plasma levels of the inflammatory cytokine interleukin-6 (IL6) were measured as described below.

\section{Source and fate of L-FABP}

From ten patients undergoing liver resection without the Pringle maneuver, arterial blood was sampled preoperatively and before liver transection. Simultaneous with the second arterial blood sample, blood was drawn from the portal vein, a hepatic vein, and the right renal vein by direct puncture to assess 


\section{Chapter 2}

concentration gradients across organs. It should be noted that at this timepoint surgical procedures were identical in all patients, irrespective of the eventual application of a Pringle maneuver. L-FABP plasma concentrations were measured as described below and arteriovenous concentration differences were calculated to study the contribution of the intestines and the liver to systemic L-FABP release and to study renal clearance of L-FABP. Renal clearance was calculated by dividing the arteriovenous concentration gradient by the arterial concentration (uptake/influx). This quotient was multiplied by the percentage of blood flowing through the kidney to calculate fractional plasma clearance.

\section{Effects of anesthesia and intestinal manipulation}

To differentiate the effects of anesthesia induction, laparotomy, and intestinal manipulation from the effects of liver manipulation, a control group consisting of four patients undergoing lower abdominal surgery (1 rectopexia, 2 proctectomy, 1 sigmoidal resection) was studied. Plasma was sampled before surgery and at 40-min intervals during surgery to determine L-FABP concentrations.

\section{Blood processing and analysis}

Blood samples were collected in prechilled EDTA vacuum tubes (BD vacutainer, Becton Dickinson Diagnostics, Aalst, Belgium) and kept on ice. Blood was centrifuged at $4000 \mathrm{~g}$ for $10 \mathrm{~min}$. Plasma was immediately stored at $-80^{\circ} \mathrm{C}$ until analysis. L-FABP and IL-6 were determined using commercially available enzyme-linked immunosorbent assays (ELISA) (kindly provided by Hycult Biotechnology, Uden, The Netherlands), GSTa was measured by ELISA as described earlier ${ }^{8,9}$. AST was determined by the clinical chemistry laboratory of the University Hospital Maastricht.

\section{Ethics}

The studies were approved by the Medical Ethics Committee of the University Hospital Maastricht and all subjects gave written informed consent.

\section{Statistics}

Normality of all data obtained was verified by Lillieford's test (all $p>0.10$ ). Data are presented as mean with standard error of the mean (SEM). A paired $t$ test was used to test the significance of changes in the various plasma concentrations. Arteriovenous concentration gradients were tested versus a theoretical mean of zero using a one-sample t test ${ }^{10}$. Pearson's test for correlations was used to test the significance of correlations. Statistical 
calculations were made using Prism 4.0 for Windows (GraphPad Software Inc., San Diego, CA). A p value less than 0.05 was considered to indicate statistical significance.

\section{Results}

\section{Patient outcome}

No signs of organ failure or other major complications were observed postoperatively during the hospital stay of all patients.

Estimation of hepatocellular injury and intestinal injury after organ manipulation

Systemic levels of damage markers

During assessment of resectability, prior to organ transection, mean arterial LFABP plasma concentration increased 55 -fold $(p=0.002)$ (Fig. 1a). Surprisingly, arterial L-FABP levels did not change significantly during subsequent intermittent Pringle maneuver, indicating that neither intermittent ischemiareperfusion nor liver transection significantly aggravated the hepatocellular injury caused by early perioperative processes. Immediately postoperatively, systemic L-FABP levels were found to be decreasing. Systemic plasma levels of GSTa (Fig. 1b) and AST (Fig. 1c) also increased significantly before liver transaction and before ischemia-reperfusion. 


\section{Chapter 2}
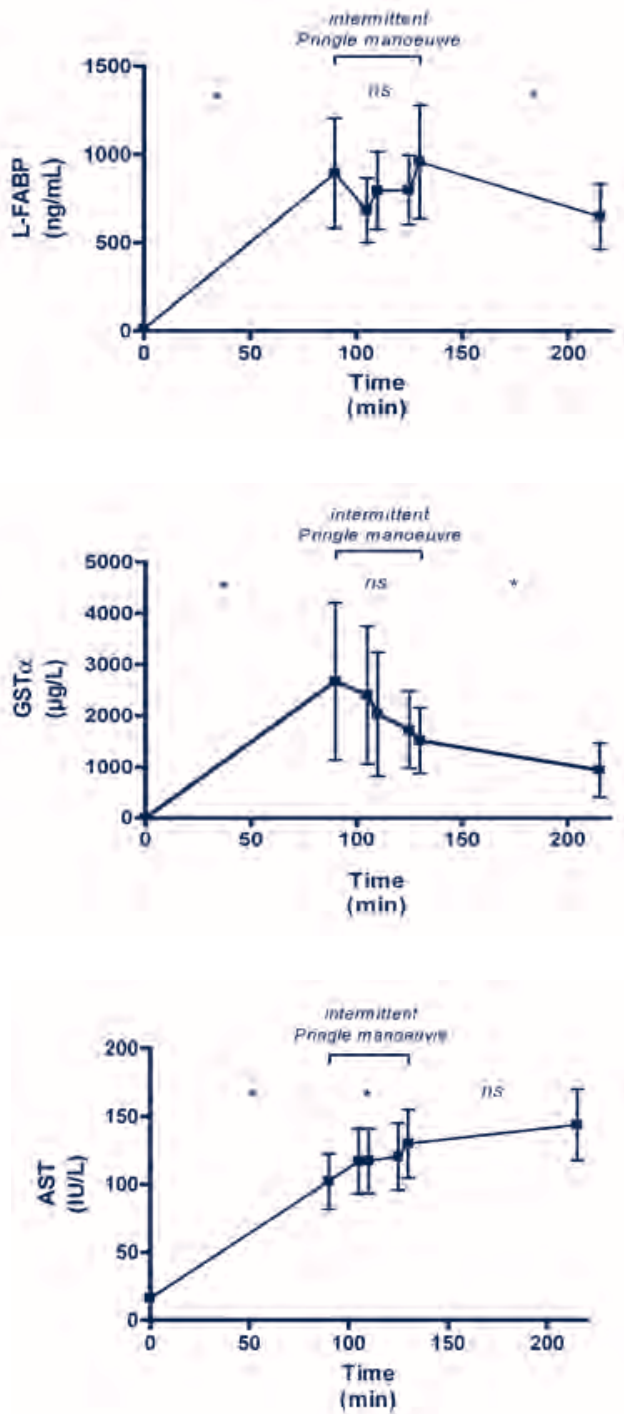

Figure 1: Mean (SEM) arterial L-FABP (a), GST $\alpha$ (b), and AST (c) levels in patients undergoing liver surgery with intermittent Pringle maneuver $(n=9)$. Plasma levels of all liver injury markers increased early during surgery, during liver manipulation $(p<0.05)$. Intermittent pringle maneuver (2x 15min ischemia and 5 min reperfusion) did not cause significant changes in arterial L-FABP and GST $\alpha$ levels, wheareas AST gradually kept increasing. L-FABP and GSTA $\alpha$ levels decreased immediately postoperatively $\left(p=0.03\right.$ and $p=0.004$, respectively). ${ }^{*} p<0.05, n s=$ not significant; $\mathrm{I}=$ ischemia, $\mathrm{R}=$ reperfusion. 


\section{Effects of lower abdominal surgery on liver injury}

In patients undergoing lower abdominal surgery, the mean LFABP concentration remained below $15 \mathrm{ng} / \mathrm{ml}$, underlining the effect of direct manipulation of the liver on the elevation of L-FABP plasma levels during liver surgery (Fig. 2).
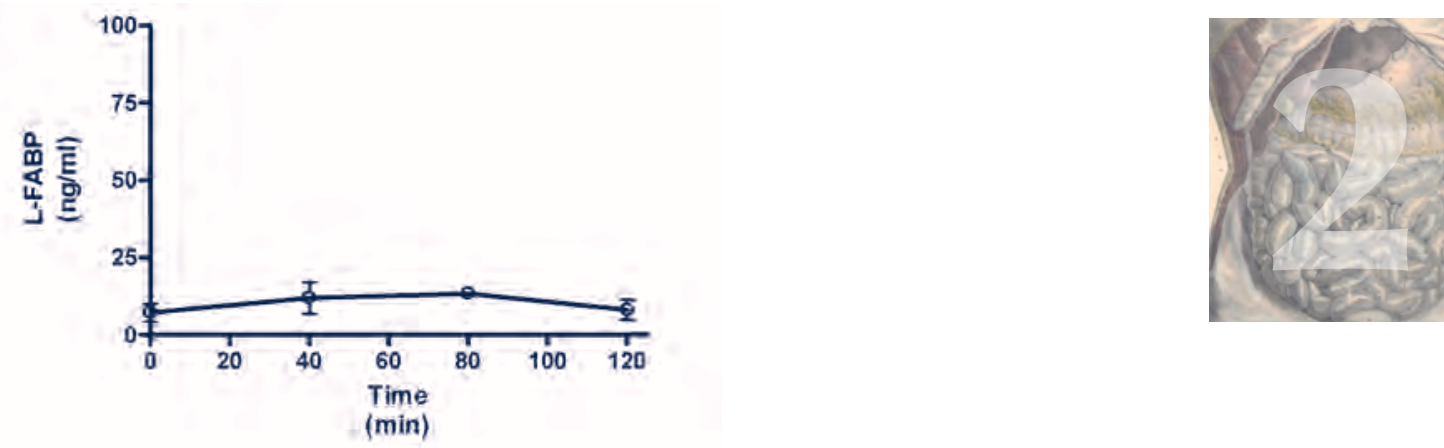

Figure 2: Mean (SEM) plasma L-FABP levels in four patients undergoing lower abdominal surgery without manipulation of the liver. No changes in L-FABP concentration were observed.

\section{Organ-specific FABP release}

Since L-FABP is expressed in both the liver and the intestines, we performed an organ balance analysis to reveal the origin of circulating L-FABP. The data show that L-FABP was specifically released from the liver and not from the intestines. In addition, organ balance analysis showed an active renal uptake of L-FABP from circulation (Fig. 3).

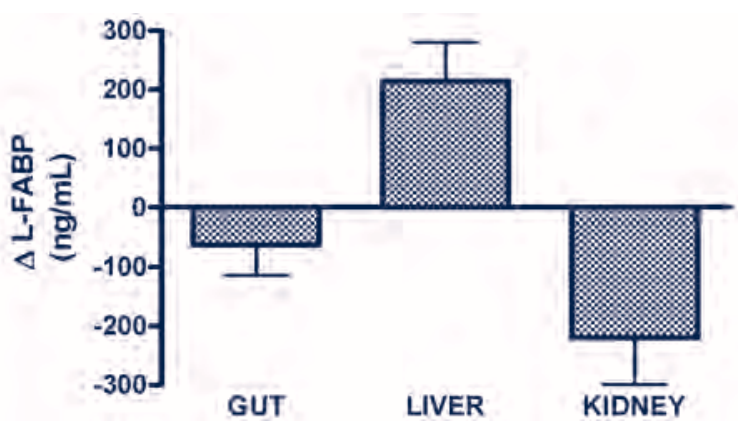

Figure 3: Mean (SEM) arteriovenous concenration gradients of L-FABP across the intestines (portal vein minus artery), the liver (hepatic vein minus portal vein), and the kidney in patients undergoing liver resection $(n=10)$. L-FABP was specifically released from the liver $(p<0.0001$ vs. zero) and removed from circulaion by the kidneys ( $p<0.0001$ vs. zero) 


\section{Chapter 2}

\section{Renal clearance of FABPs}

The kidneys efficiently removed L-FABP from circulation. Renal clearance of LFABP correlated directly with their respective arterial concentrations (Fig. 4), resulting in a fractional extraction rate (arteriovenous gradient/arterial concentration $\cdot 100 \%$ ) [11] of approximately $30 \%$. Assuming that renal blood flow per minute approximates $22 \%$ of the total blood volume [12], renal plasma L-FABP clearance can be calculated to equal $6.6 \% / \mathrm{min}(30 \%$. $22 \% / \mathrm{min})$, resulting in a plasma half-life of about $11 \mathrm{~min}$.

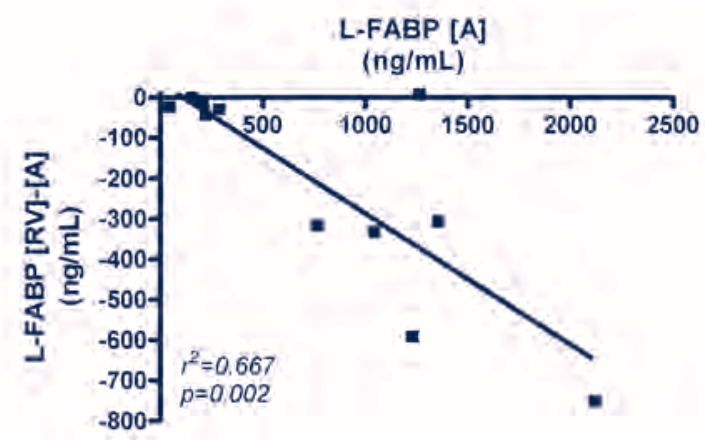

Figure 4: Fractional renal clearance of L-FABP in patients undergoing liver resection $(n=10)$. From the fractional renal extraction of FABPs (approximately $30 \%$ ) and renal blood flow (22\% of total blood volume per minute) [12], a plasma half life of FBAPs off $11 \mathrm{~min}$ can be calculated. [A] = arterial concentration, $[\mathrm{RV}]=$ renal venous concentration. 


\section{Interleukin-6 levels during liver surgery with intermittent Pringle maneuver}

The inflammatory response to liver surgery was investigated using IL-6 as a parameter. Arterial IL-6 levels remained unaltered during manipulation of the liver, when L-FABP, AST, and GSTa levels were increasing. In contrast, a significant increase of systemic IL-6 plasma levels was found after $15 \mathrm{~min}$ of hepatic inflow occlusion (Fig. 5). This increase was progressive throughout the remainder of the study period.

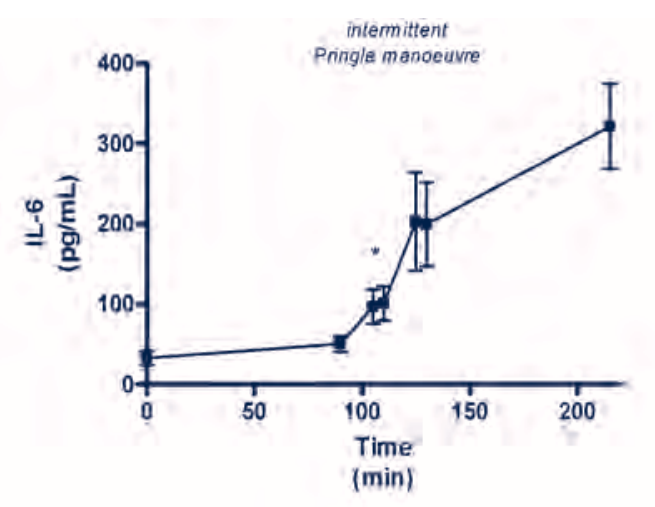

Figure 5: mean (SEM) arterial IL - 6 concentration before, during, and after live resection with intermittent Pringle maneuver $(n=9) .{ }^{*}=$ first timepoint where $p<0.05$ vs. baseline;.

\section{Discussion}

The present study was designed to determine the cause of early hepatocellular injury during liver resection. Previous publications already showed elevated plasma levels of markers of liver damage prior to liver transection and hepatic inflow occlusion ${ }^{3,6}$. These observations suggested that mechanisms other than ischemia-reperfusion injury could contribute to peri- and postoperative cell death and organ dysfunction. We showed that direct manipulation of the liver during surgery is a leading cause of hepatocellular injury.

Arterial L-FABP and GSTa plasma levels increased following the start of the operation and reached a plateau before liver transection and ischemiareperfusion. Surprisingly, we found no additional effect of organ transection or intermittent Pringle maneuver on these increased LFABP and GSTa plasma levels. The apparent resistance of the liver to an ischemic insult however, is in line with previous data by Figueras et al. ${ }^{13}$ who showed no effect of the extent of inflow occlusion in patients with a normal liver, although in their study livers of patients with cirrhosis appeared to be more vulnerable to hepatic inflow 


\section{Chapter 2}

occlusion. In addition, other authors who showed a progressive release of GSTa following a Pringle maneuver applied prolonged and continuous inflow occlusion ${ }^{6}$, which is known to aggravate hepatocellular injury compared to the intermittent Pringle maneuver ${ }^{14}$.

Previous authors who observed an early increase in plasma concentrations of transaminases and GSTa have ascribed this phenomenon to the hepatotoxic effects of anesthesia ${ }^{4}$, systemic inflammation after intestinal manipulation ${ }^{5}$, and the effects of manipulation of the liver itself during perihepatic dissection and mobilization ${ }^{6}$. We were able to rule out the first two factors as potential causes of early hepatocyte injury since a similar increase of L-FABP plasma concentrations did not occur in patients undergoing lower intestinal surgery. These patients were anesthetized in a similar manner as the patients undergoing liver resection and underwent extensive intestinal manipulation.

By elimination of other potential causes, it thus must be concluded that increasing L-FABP levels occurs during and due to liver manipulation in patients undergoing liver resection. By measuring concentration gradients across the intestines and the liver, we were able to show that the increased LFABP levels during liver manipulation are exclusively due to L-FABP release from the liver. Some intestinal L-FABP release could have been expected when L-FABP, which is also expressed by the intestines, would have been released from injured enterocytes, but the absence of intestinal L-FABP release clearly indicates that organ manipulation during liver resection results in hepatocellular injury without causing intestinal injury.

Finally, we were able to rule out systemic inflammation as a leading cause of hepatocellular injury since L-FABP peak levels were reached already before the onset of the inflammatory response. Arterial IL-6 plasma levels increased between 90 and 125 min after laparotomy, which approximates the established time lag between an inflammatory stimulus and IL- 6 release ${ }^{15}$. This suggests that the inflammatory response is triggered by an early event during the operation.

According to the "danger model" hypothesis of Matzinger ${ }^{16}$, cell injury leads to the release of immunostimulatory proteins or nucleotides, so-called danger signals, that activate the immune system and induce systemic inflammation ${ }^{17}$. In line with this theory, we consider that early hepatocyte damage due to liver manipulation could give rise to the release of such "danger signals" and contribute to systemic inflammation. Consequently, liver manipulation-induced hepatocyte injury may be a trigger for the inflammatory response to surgery. Mechanistic proof for this theory, however, is difficult to obtain in vivo.

Both L-FABP and GSTa decreased immediately following surgery, in contrast to 
the more classic marker of hepatocyte injury, AST. The ongoing increase of plasma levels of such markers following liver surgery has been regarded as a sign of ongoing hepatocellular injury and impending liver failure ${ }^{18}$. Our study shows that the late postoperative peak of AST is more likely to be a reflection of slow leakage than of ongoing injury, since the leakage of small-molecular proteins L-FABP and GSTa decreased within 90 min. As a consequence, L-FABP and GSTa are probably more sensitive for detecting ongoing hepatocyte injury and impending liver failure than AST. To prove this assumption, however, a large prospective study is needed. The rapid decline of L-FABP and GSTa is a result of their rapid renal clearance. Arterial renal venous concentration gradients showed that the kidneys remove approximately $30 \%$ of L-FABP in a single pass, leading to a calculated LFABP half-life of $11 \mathrm{~min}$.

We did not explore potential mechanisms of liver manipulation-induced cell injury. Earlier studies showed that liver mobilization and assessment of resectability significantly reduced hepatic venous oxygen saturation ${ }^{19,20}$. This could also be a cause of hepatocyte damage in our case, although we did not measure hepatic oxygen saturation. It is not clear whether hepatic oxygen saturation decreased due to physical obstruction of the blood stream or whether it decreased secondary to other processes 21,22 . Alternatively, cell damage may occur as a direct consequence of mechanical impact ${ }^{23}$.

In summary, it was previously believed that vascular occlusion and parenchymal transection were the major reasons for hepatocyte injury during liver surgery. This study demonstrates that liver manipulation is a leading cause of hepatocyte injury during liver surgery. A potential causal relation between liver manipulation and systemic inflammation remains to be established. However, since the inflammatory cascade is apparently initiated early during major abdominal surgery, interventions aimed at reducing postoperative inflammation and related complications should be started early during surgery or beforehand. 


\section{Chapter 2}

\section{RefERENCES}

1. Wigmore SJ, Redhead DN, Yan XJ, et al. (2001) Virtual hepatic resection using threedimensional reconstruction of helical computed tomography angioportograms. Ann Surg 233:221-226

2. Patel A, van de Poll MC, Greve JW, et al. (2004) Early stress protein gene expression in a human model of ischemic preconditioning. Transplantation 78:1479-1487

3. Boschetto A, Dondero F, Tonini V, et al. (2005) Intra-operative liver injury is not only related to vascular clamping [abstract]. HPB 7:P25

4. Clarke RS, Doggart JR, Lavery T (1976) Changes in liver function after different types of surgery. Br J Anaesth 48:119-128

5. Hiki N, Shimizu N, Yamaguchi H, et al. (2006) Manipulation of the small intestine as a cause of the increased inflammatory response after open compared with laparoscopic surgery. Br J Surg 93:195-204

6. Chouker A, Martignoni A, Schauer RJ, et al. (2005) a-Gluthathione s-transferase as an early marker of hepatic ischemia/reperfusion injury after liver resection. World I Surg 29:528-534

7. Dejong CHC, Garden OJ (2003) Neoplasms of the liver. In: Majid AA, Kingsnorth A (eds) Advanced surgical practice. London, Greenwich Medical Media, pp 146-156

8. Mulder TP, Peters WH, Court DA, et al. (1996) Sandwich ELISA for glutathione Stransferase alpha 1-1: plasma concentrations in controls and in patients with gastrointestinal disorders. Clin Chem 42:416-419

9. Steegers EA, Mulder TP, Bisseling JG, et al. (1995) Glutathione S-transferase alpha as marker for hepatocellular damage in preeclampsia and HELLP syndrome. Lancet 345:1571-1572

10. Motulsky HJ (2003) Comparing a mean or median to a theoretical value. Prism 4 Statistics Guide: Statistical analyses for laboratory and clinical researchers. GraphPad Software Inc., San Diego, CA, pp 35-39

11. Van de Poll MC, Siroen MP, van Leeuwen PA, et al. (2007) Interorgan amino acid exchange in humans: consequences for arginine and citrulline metabolism. Am J Clin Nutr 85:167-172

12. Riddez L, Hahn RG, Brismar B, et al. (1997) Central and regional hemodynamics during acute hypovolemia and volume substitution in volunteers. Crit Care Med 25:635-640

13. Figueras J, Llado L, Ruiz D, et al. (2005) Complete versus selective portal triad clamping for minor liver resections: a prospective randomized trial. Ann Surg 241:582-590

14. Belghiti J, Noun R, Malafosse R, et al. (1999) Continuous versus intermittent portal triad clamping for liver resection: a controlled study. Ann Surg 229:369-375 
15. Lin E, Calvano SE, Lowry SF (2000) Inflammatory cytokines and cell response in surgery. Surgery 127:117-126

16. Matzinger P (2002) The danger model: a renewed sense of self. Science 296:301-305

17. Barrat FJ, Meeker T, Gregorio J, et al. (2005) Nucleic acids of mammalian origin can act as endogenous ligands for Toll-like receptors and may promote systemic lupus erythematosus. J Exp Med 202:1131-1139

18. Panis Y, McMullan DM, Emond JC (1997) Progressive necrosis after hepatectomy and the pathophysiology of liver failure after massive resection. Surgery 121:142-149

19. Kretzschmar M, Kruger A, Schirrmeister W (2003) Hepatic ischemia-reperfusion syndrome after partial liver resection (LR): hepatic venous oxygen saturation, enzyme pattern, reduced and oxidized glutathione, procalcitonin and interleukin-6. Exp Toxicol Pathol 54:423-431

20. Kainuma M, Fujiwara Y, Kimura N, et al. (1991) Monitoring hepatic venous hemoglobin oxygen saturation in patients undergoing liver surgery. Anesthesiology 74:49-52

21. Schemmer P, Enomoto N, Bradford BU, et al. (2001) Activated Kupffer cells cause a hypermetabolic state after gentle in situ manipulation of liver in rats. Am J Physiol Gastrointest Liver Physiol 280:G1076-1082

22. Schemmer P, Enomoto N, Bradford BU, et al. (2001) Autonomic nervous system and gut-derived endotoxin: involvement in activation of Kupffer cells after in situ organ manipulation. World J Surg 25:399-406

23. Lazarowski ER, Homolya L, Boucher RC, et al. (1997) Direct demonstration of mechanically induced release of cellular UTP and its implication for uridine nucleotide receptor activation. J Biol Chem 272:24348-24354 
Chapter 2

\subsection{Urine based detection of intestinal tight junction loss}

Geertje Thuijls, Joep P. M. Derikx, Jacco-Juri de Haan, Joep Grootjans, Adriaan de Bruïne, Ad A. M. Masclee, Erik Heineman, Wim A. Buurman Journal of Clinical Gastroenterology, in press. 


\begin{abstract}

\section{Background}

Tight junction breakdown, with loss of the important sealing protein claudin-3, is an early event in the development of intestinal damage. Therefore noninvasive analysis of intestinal tight junction status could be helpful in early detection of intestinal injury.
\end{abstract}

\begin{abstract}
Aim
To investigate the usefulness of urinary claudin-3 as marker for intestinal tight junction loss.
\end{abstract}

\title{
Methods
}

A rat hemorrhagic shock model and a human setting of known intestinal damage, i.e. patients with relapsed inflammatory bowel disease (IBD), were used to investigate intestinal tight junction status by immunohistochemical staining and urinary claudin-3 levels by western blot.

\section{Results}

In rats claudin-3 urine levels increased rapidly after histologically proven intestinal tight junction loss, with significantly elevated levels at 90 minutes after shock compared to sham operated animals (mean \pm SEM: 611 \pm 101 intensity (INT), $n=6$ versus $232 \pm 30$ INT, $n=6 ; p<0.05)$.

Moreover, in colonic biopsies of patients with IBD relapse claudin-3 staining was reduced compared to biopsies of patients with IBD without signs of disease. Concomitantly, significantly increased claudin-3 urine levels were found in these patients $(502 \pm 67$ INT, $n=10)$ compared to patients with IBD in remission $(219 \pm 17$ INT, $n=10, p<0.001)$ and healthy volunteers $(225 \pm 38$ INT, $\mathrm{n}=10, \mathrm{p}<0.001)$.

\section{Conclusion}

Here we show for the first time in both an experimental and clinical setting a strong relation between intestinal tight junction loss and urinary claudin-3 levels. These findings suggest that measurement of urinary claudin-3 can be used as non-invasive marker for intestinal tight junction loss. This offers new opportunities for early diagnosis and follow-up of intestinal injury. 


\section{INTRODUCTION}

Gut wall integrity loss, including breakdown of tight junctions, is an early event in intestinal damage. ${ }^{1-3}$ Currently, invasive intestinal biopsies provide the only possibility to detect tight junction breakdown. ${ }^{2}$ Since endoscopy for biopsy is a time-consuming and invasive procedure, a non-invasive method for detection of tight junction breakdown would facilitate detection of tight junction loss. Such assay could help in early diagnosis of disease in patients with intestinal damage, including neonates suspected for necrotizing enterocolitis, patients suspected for inflammatory bowel disease or celiac disease. Reliable markers for intestinal tight junction loss will also be useful for follow-up of patients with intestinal damage, for evaluation of therapy effects and for prediction of disease relapse. Moreover, markers for tight junction integrity loss can be helpful in the elucidation of the pathophysiology of diseases in which injury of the gut plays a role.

Tight junctions serve as a fence separating the internal environment of the host from the gut luminal content, thereby preventing exposure of the internal milieu to potentially harmful microbiota and microbial products. Tight junctions consist of a large complex of proteins, including claudins $(22 \mathrm{kDa})$ which are mainly responsible for the intestinal barrier function and are present in abundance between adjacent healthy intestinal epithelial cells. ${ }^{1-5}$ Especially claudin-3 is an important sealing claudin, expressed in high quantity in the intestine. ${ }^{4}$ The amount of claudin-3 in the intestine decreases following tight junction integrity loss. ${ }^{2}$ The small size, the abundant endogenous intestinal expression and the paracellular localisation make claudin-3 a suitable candidate as urinary marker for early non-invasive detection of intestinal tight junction integrity loss. The aim of this pilot study is to investigate whether intestinal tight junction loss results in the appearance of claudin-3 in the urine and subsequently to assess the potential usefulness of claudin-3 as urine marker for the non-invasive detection of intestinal tight junction loss.

\section{Methods}

\section{Experimental design}

A rat hemorrhagic shock (HS) model and a human setting of intestinal disease with known tight junction loss were studied. Both studies are conducted after approval of the local ethical committees (Animal Care Committee of Maastricht University and Medical Ethical Review Board of Maastricht University Medical Centre). 


\section{Rat hemorrhagic shock procedure}

Sprague-Dawley rats were allocated to 5 groups ( $n=6$ per group) before the start of the experiments. Control rats (group 1) were sacrificed without intervention. The other rats were exposed to non-lethal HS after 18 hours fasting as previously described. ${ }^{6}$ Briefly, rats were anesthetized with isoflurane (induction $4 \%$, maintenance $1.5 \%$ ). The femoral artery was aseptically dissected and cannulated with polyethylene tubing (PE-10) containing heparinized saline (10 $\mathrm{IU} / \mathrm{ml})$. Mean arterial blood pressure (MAP) and heart rate (HR) were assessed continuously. After 30 minutes acclimatization period, $2.1 \mathrm{~mL}$ blood /100 g of body weight was withdrawn (representing approximately $30-40 \%$ of the circulating volume) at a rate of $1 \mathrm{~mL} /$ minute. Groups 2, 3, 4, and 5 were sacrificed at 15, 30, 60 and 90 minutes after shock, respectively. At sacrifice urine and tissue samples were taken. The severity of the HS as reflected by changes in MAP, HR, and hematocrit was similar for all animals studied. These data are comparable with previously published data, using the same HS model. ${ }^{7,8}$

\section{Human setting of intestinal disease}

Urine samples were obtained after informed consent from 10 patients with active IBD (4 Crohn's disease, 6 ulcerative colitis) (4 male, 6 female), with a median age of 37 years (range 10-68 years). Ten urine samples of healthy volunteers (4 male, 6 female), median age 30 years (range 27-60 years), and 10 urine samples of patients with IBD in remission (5 Crohn's disease, 5 ulcerative colitis) (5 male, 5 female), median age 38 years (range 15-61 years) were studied. Colonic biopsies of patients with IBD were taken for routine patient care and used for this study to assess histological tight junction status.

\section{Immunohistochemistry for claudin-3}

Immunohistochemistry for claudin-3 on rat colon and human colonic biopsies was performed. Briefly, rat colon and human colonic biopsies, fixed overnight in $4 \%$ formaldehyde, were embedded in paraffin and sectioned at $3 \mu \mathrm{m}$. The section were deparaffinised in xylol and endogenous peroxidases were blocked in 3\% H2O2/methanol. Sections were stained for 1 hour at room temperature with Rabbit anti-Claudin-3 ((34-1700), Zymed Laboratories, San Francisco, CA). This was followed by 30 minutes incubation with poly-HRP-GAM/R/R IgG (ImmunoLogic, Duiven, the Netherlands) and visualized by applying diaminobenzidine (DAB) (Dakocytomation, Glostrup, Denmark). Nuclei were counterstained with haematoxylin. The distribution of claudin-3 was recorded at a magnification of 400x using the Metasystems Image Pro System (black and 


\section{Chapter 2}

white charge-couple device camera; Metasystems, Sandhausen, Germany) mounted on a Leica DM-RE microscope (Leica, Wetzler, Germany). All images were taken at equal time-exposures after being normalized to negative control sections without primary antibody, to exclude for non-specific binding of the secondary antibody. At least 25 microscopic fields for each tissue section were examined.

\section{Western blot for urinary claudin-3}

Claudin-3 urine levels were analyzed by western blotting. First, to correct for urinary dilution all urine samples were adjusted to urinary creatinine levels. Creatinine is a well established marker reflecting the degree of urinary dilution. Second, equal amounts of each sample (adjusted to urinary creatinine levels) were separated by SDS-PAGE gel, transferred to PVDF-membrane and probed using primary antibody to claudin-3 (Rabbit anti-Claudin-3 (34-1700), Zymed Laboratories, San Francisco, CA). After incubation with goat anti rabbit HRPconjugated secondary antibody (Jackson, West Grove, PA), signal was detected by supersignal west pico chemiluminescence substrate (Pierce, Etten-Leur, the Netherlands). Band intensity was semi-quantitatively analyzed using Quantity One (Biorad, Hercules, CA).

\section{Statistical analysis}

Gaussian distribution was tested using the Kolmogorov-Smirnov test. One-way ANOVA, with post-hoc Bonferroni multiple comparison test, was used for between group comparison. $\mathrm{P}<0.05$ was considered statistically significant. Data are expressed as mean \pm SEM of intensity using an arbitrary measure (INT).

\section{Results}

\section{Intestinal claudin-3 loss}

Immunostaining of claudin-3 was seen in junctional and basolateral cell membranes in the colon of control rats. Hemorrhagic shock led to a significant reduction of claudin-3 staining in the colon in animals sacrificed 60 minutes after the induction of shock (Fig. 1A).

In line with the animal data, less claudin-3 staining was observed in colonic biopsies of patients with IBD relapse, mainly at the junctional membranes, compared to colonic biopsies of patients with IBD without signs of disease (Fig. 1B). These data indicate that the protein claudin-3 is lost upon intestinal injury. 

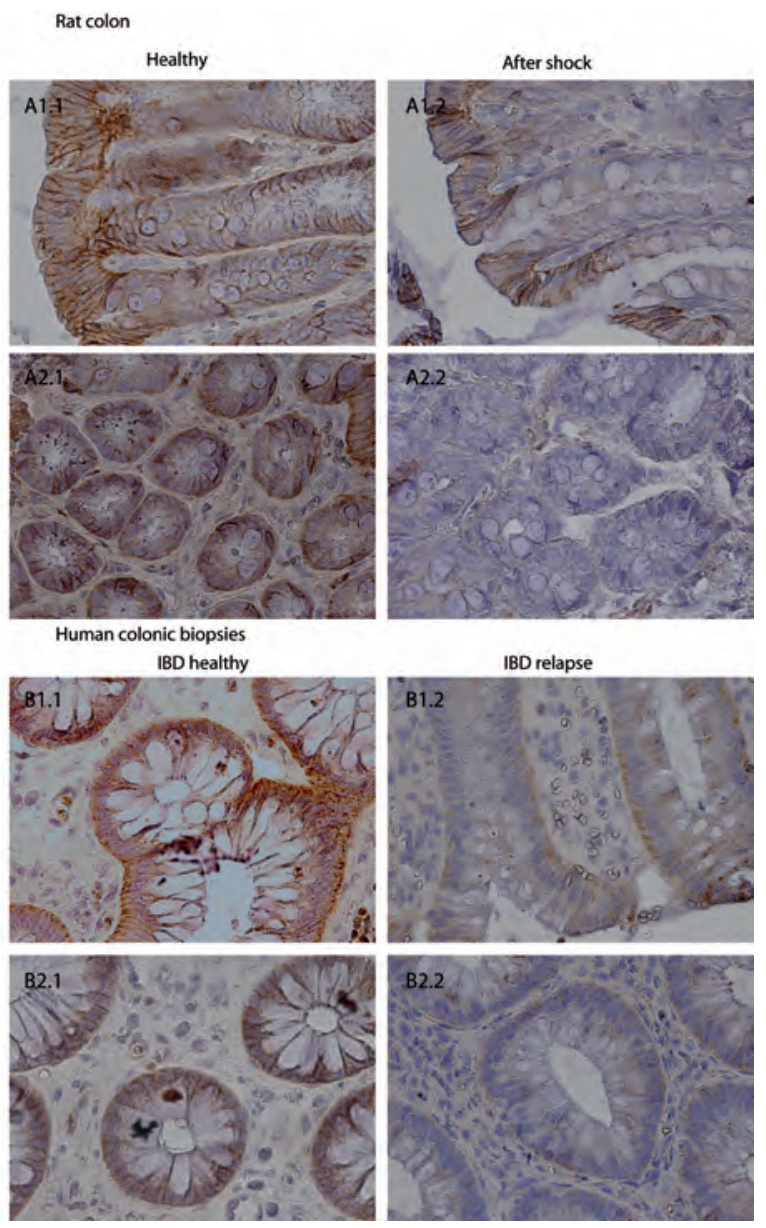

Figure 1: (A) Representative photographs of claudin-3 (DAB-stained) in longitudinal (A1.) and transversal (A2.) colonic sections of healthy rats and rats sacrificed 60 minutes following induction of shock. Claudin-3 is present in the colon of control rats in junctional and basolateral cell membranes (A1.1 + A2.1). After hemorrhagic shock, staining is reduced (A1.2 + A2.2). N=6 per group, 400x magnification.

(B) Representative photographs of claudin-3 (DAB-stained) in longitudinal (B1.) and transversal (B2.) colonic biopsy sections of patients with IBD (ulcerative colitis). Claudin-3 (DAB-stained) is present in junctional and basolateral cell membranes in colonic biopsies of patients with IBD without signs of disease (B1.1 + B2.1). Reduced staining of claudin-3 in colonic biopsies of patients with IBD relapse was observed, mainly at the junctional membranes of the epithelial cell $(B 1.2+B 2.2) . \mathrm{N}=4$ per group, 400x magnification. 


\section{Chapter 2}

\section{Increased urinary claudin-3 levels}

Western blot analysis showed the presence of claudin-3 in urine of sham operated rats $(232 \pm 30$ INT). Significantly increased claudin-3 levels were detected in urine, corrected for urinary concentration occurring in shock, at 90 minutes after induction of hemorrhagic shock $(611 \pm 101$ INT, $p<0.05)$, following histologically proven loss of intestinal claudin-3 observed at 60 minutes after induction of hemorrhagic shock (Fig. 2).

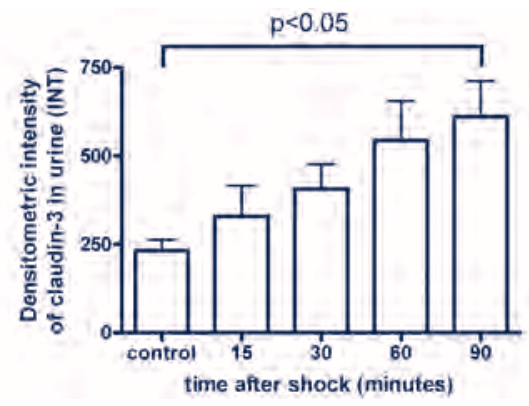

Figure 2: A significant increase in claudin-3 urine levels is detected in rats sacrificed 90 minutes after shock compared to controls, reflecting intestinal tight junction loss. Values are expressed as mean \pm SEM, $\mathrm{n}=6$ per group.

Also in the human setting, significantly increased claudin-3 urine levels were found in patients with IBD relapse $(502 \pm 67$ INT) compared to healthy volunteers $(225 \pm 38$ INT, $\mathrm{p}<0.001)$ and to patients with IBD in remission $(219$ \pm 17 INT, $p<0.001$ ) (Fig. 3), which was in line with reduced claudin-3 staining in patients with IBD relapse, compared to patients with IBD in remission.

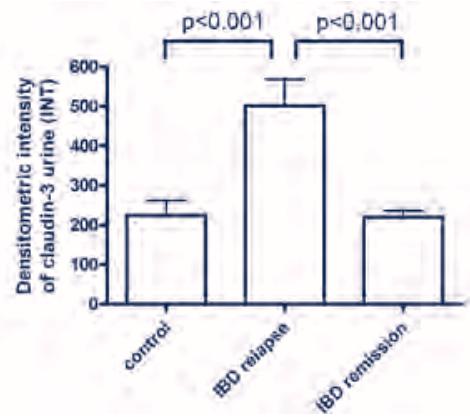

Figure 3: A significant increase in claudin-3 urine levels in patients with active IBD compared to healthy volunteers and patients with IBD in remission was observed, indicating that tight junction loss was detectable in urine. Values are expressed as mean \pm SEM, $n=10$ per group. 


\section{Discussion}

Non-invasive detection of tight junction loss could help in early diagnosis and follow-up of patients with intestinal diseases like necrotizing enterocolitis, IBD or celiac disease.

To evaluate the usefulness of urinary claudin-3 as non-invasive marker to assess intestinal tight junction loss we first used an experimental animal model with proven tight junction loss. ${ }^{6,9}$ Recently we observed in this experimental rat HS model that intestinal barrier loss is present without systemic inflammation within 90 minutes after induction of hemorrhagic shock (manuscript in preparation). To rule out the effect of systemic inflammation on tight junction status, because systemic inflammation can lead to tight junction loss in other organs besides the gut, we used this HS model to investigate whether intestinal tight junction loss results in the appearance of claudin-3 in the urine. To translate our data to a clinically relevant human setting, patients with IBD were included.

This pilot study shows that intestinal claudin-3 immunostaining was decreased in both an animal and a human model for intestinal damage, reflecting loss of claudin-3 upon intestinal barrier damage. More importantly, urinary claudin-3 levels were elevated when intestinal tight junction loss occurred, strongly indicating that elevated urinary claudin-3 levels reflect loss of tight junctions.

Although, it has previously been shown that besides in the intestine, claudin-3 is also expressed in tight junctions of liver, kidney and lung. ${ }^{5}$ We recently showed in the same experimental rat $\mathrm{HS}$ model that no significant loss of claudin-3 occurred in the liver and kidney.9 In addition, others showed that HS does not result in early pulmonary tight junction loss, in absence of severe associated factors, like massive inflammation. ${ }^{10,11}$

In this study, immunohistochemistry was performed on intestinal tissue specimens to objectify loss of claudin-3 at a histological level, showing reduced claudin-3 staining in the intestinal specimens after induction of HS and in patients with IBD relapse. Therefore we consider that the urinary claudin-3 levels reflect intestinal tight junction loss. The amount of claudin-3, detected in urine of sham operated rats and healthy human volunteers, is most likely related to the level of turnover of intestinal epithelium.

The observed increase of urine claudin-3 after immunohistologically observed intestinal claudin-3 reduction indicates loss of claudin-3 into the blood circulation and subsequent clearance in urine. In contrast to the current data, a number of previous studies show internalization of tight junction 
proteins, including claudins during paracellular barrier loss, intercellular movement and epithelial turnover ${ }^{12,13}$ We suppose that during epithelial turnover as well as during barrier loss both internalization of claudins and claudin loss and successive urinary excretion take place. The latter is supported by the decreased intestinal expression of claudin-3 after hemorrhagic shock on western blot as we showed in a previous study. ${ }^{9}$

To translate the animal data to a human setting, colonic biopsies and urine of patients with objectified intestinal disease, i.e. patients with relapsed inflammatory bowel disease (IBD), were studied. First, we showed reduced intestinal tight junction staining in patients with IBD relapse. This is supported by recent findings of Zeissig et al. who showed disturbance of barrier function and reduced presence of claudin proteins including claudin-3, in intestinal biopsies of patients affected with IBD. ${ }^{2}$ Second, concomitant to the reduced intestinal claudin-3 staining, we showed elevated urinary claudin-3 levels. These preliminary data suggest that urinary claudin-3 can be used to detect intestinal tight junction loss in the human setting. Since urinary claudin-3 measurement is not specific for intestinal tight junction loss, this test should be performed based on clinical arguments.

In conclusion; we show for the first time in both an experimental and a clinical setting a strong relation between intestinal tight junction loss and urinary claudin-3 levels. These findings suggest that measurement of urinary claudin-3 can be used as non-invasive marker for intestinal tight junction loss. This offers new possibilities for early diagnosis and follow-up of patients with intestinal diseases by detection of tight junction loss. Furthermore, the ability to measure claudin-3 urinary levels could contribute to the elucidation of the pathophysiology of gut related diseases in man. 


\section{References}

1. Fink MP, Delude RL. Epithelial barrier dysfunction: a unifying theme to explain the pathogenesis of multiple organ dysfunction at the cellular level. Crit Care Clin. 2005; 21:177-96.

2. Zeissig S, Burgel N, Gunzel D, et al. Changes in expression and distribution of claudin 2, 5 and 8 lead to discontinuous tight junctions and barrier dysfunction in active Crohn's disease. Gut. 2007;56:61-72.

3. Suenaert P, Bulteel V, Lemmens L, et al. Anti-tumor necrosis factor treatment restores the gut barrier in Crohn's disease. Am J Gastroenterol. 2002;97:2000-4.

4. Rahner C, Mitic LL, Anderson JM. Heterogeneity in expression and subcellular localization of claudins 2, 3, 4, and 5 in the rat liver, pancreas, and gut. Gastroenterology. 2001;120:411-22.

5. Turksen K, Troy TC. Barriers built on claudins. J Cell Sci. 2004;117:2435-47.

6. Luyer MD, Buurman WA, Hadfoune M, et al. Pretreatment with high-fat enteral nutrition reduces endotoxin and tumor necrosis factor-alpha and preserves gut barrier function early after hemorrhagic shock. Shock. 2004;21:65-71.

7. Bark T, Katouli M, Ljungqvist $\mathrm{O}$, et al. Bacterial translocation after non-lethal hemorrhage in the rat. Circ Shock. 1993;41:60-5.

8. Luyer MD, Jacobs JA, Vreugdenhil AC, et al. Enteral administration of high-fat nutrition before and directly after hemorrhagic shock reduces endotoxemia and bacterial translocation. Ann Surg. 2004;239:257-64.

9. Thuijls G, de Haan J, Derikx JP, et al. Intestinal Cytoskeleton Degradation Precedes Tight Junction Loss Following Hemorrhagic Shock. Shock. 2008.

10. Deitch EA, Forsythe R, Anjaria D, et al. The role of lymph factors in lung injury, bone marrow suppression, and endothelial cell dysfunction in a primate model of traumahemorrhagic shock. Shock. 2004;22:221-8.

11. Peitzman AB, Billiar TR, Harbrecht BG, et al. Hemorrhagic shock. Curr Probl Surg. 1995;32:925-1002.

12. Ivanov AI, Nusrat A, Parkos CA. The epithelium in inflammatory bowel disease: potential role of endocytosis of junctional proteins in barrier disruption. Novartis Found Symp. 2004;263:115-24; discussion 124-32, 211-8.

13. Matsuda M, Kubo A, Furuse M, et al. A peculiar internalization of claudins, tight junction-specific adhesion molecules, during the intercellular movement of epithelial cells. J Cell Sci. 2004;117:1247-57. 


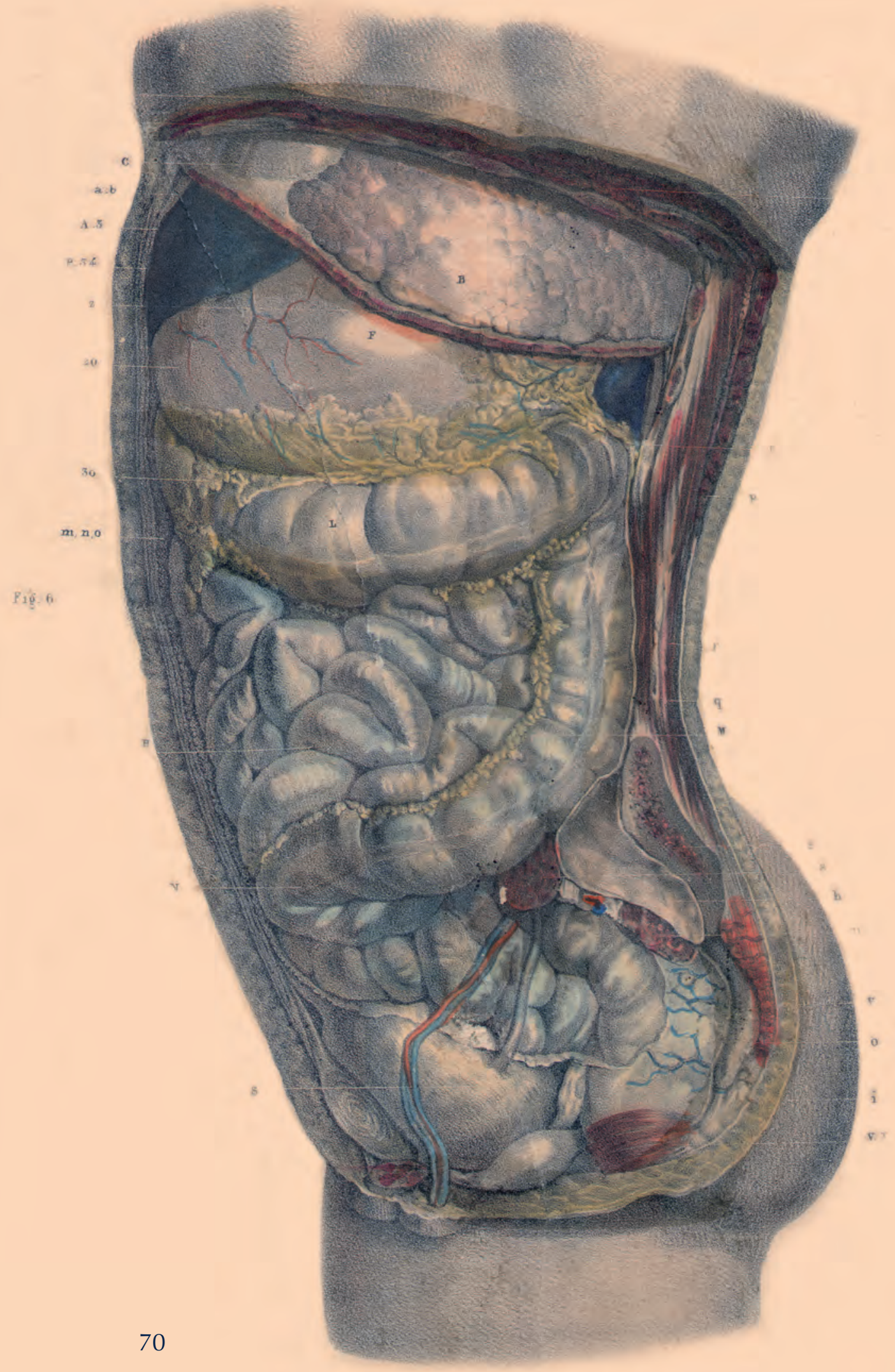




\section{ChaPter 3}

Analysis of markers for compromised gut wall in patients with overt gut damage and in patients with suspected intestinal disease to distinguish the target disease from other pathologies 


\section{Chapter 3}

\subsection{INTRODUCTION}

To investigate the second aim: i.e. analysis of markers for compromised gut wall in patients with overt gut damage and in patients with suspected intestinal disease to distinguish the target disease from other pathologies, the usefulness of non-invasive tests for enterocyte damage, TJ disruption and gut wall inflammation was evaluated. These human studies regarded different phases of diagnostic studies. Diagnostic tests aim at discriminating between clinically "normal" and "abnormal". New diagnostic tests should subsequently answer the following 4 questions before implementation of the new test in daily clinical practice can be contemplated ${ }^{1}$. Question 1: Do patients with the target disorder have different test results compared to normal individuals? The answer requires comparison of test results among individuals with the disease and individuals that are disease-free. This question was piloted in a child receiving high-dose chemotherapy (paragraph 3.2) and in a study with adult patients receiving myeloablative conditioning for haematopoietic stem cell transplantation (HSCT) (paragraph 3.3). A major risk in these patients is the development of mucosal barrier injury, potentially resulting in serious complications. The identification of mucosal barrier injury in patients before and after myeloablative conditioning was investigated using plasma levels of FABP. In another $n=1$ study, the potential development of intestinal epithelial cell damage was monitored using plasma FABP measurement in a child undergoing gastroschisis-repair (paragraph 3.4). Question 2: Are patients with certain test results more likely to have the target disorder than patients with other test results? This question is also answered by comparison of test results among patients with the targetdisease and healthy individuals, but now test characteristics as sensitivity, specificity and likelihood ratios are estimated. This phase was applied in the study concerning patients with celiac disease and in patients with appendicitis. In patients with celiac disease the diagnostic accuracy of circulating levels of FABP was investigated and in patients with appendicitis the usefulness of neutrophil activation products was studied (paragraph 2.2 and 3.5). Question 3: Among patients in whom it is clinically sensible to suspect the target disorder, does the test result distinguish those with and without the target disorder? To get the appropriate answer, a consecutive series of such patients should be studied. This phase was performed in evaluating diagnostic tests in the study of neonates suspected for necrotizing enterocolitis (NEC), since the results of phase 1 and 2 studies were already reported by other authors. Neonates admitted to our neonatal intensive care unit, suspected for NEC, were consecutively included in the study (paragraph 
3.6 and 3.7). Aim of the study was to differentiate NEC from other neonatal diseases that present with abdominal signs and symptoms suspicious of NEC using non-invasive tests for enterocyte damage (urinary FABP), TJ disruption (urinary claudin-3) and gut wall inflammation (faecal calprotectin). Question 4: Do patients who undergo the diagnostic test fare better (in their ultimate health outcomes) than similar patients who do not? The question has to be answered by randomizing patients to undergo the test of interest or another (or no) test. No study was performed regarding phase 4 questions.

\section{REFERENTIES}

1. Sackett DL, Haynes RB. The evidence base of clinical diagnosis. London: BMJ Publishing Group, 2002. 


\subsection{Detection of chemotherapy induced enterocyte toxicity with circulating Intestinal Fatty Acid Binding Protein}

Joep P. M. Derikx, Dick A. van Waardenburg, Bernd Granzen, Annemarie A. van Bijnen, Erik Heineman, Wim A. Buurman. Journal of Pediatric Hematology and Oncology (2006) 28: 267-9. 


\begin{abstract}

\section{Background}

Cytotoxic gastro-intestinal (GI) mucositis is a difficult to diagnose complication of chemotherapy. It contributes potentially to local invasion by microorganisms and, subsequently, to systemic infection in neutropenic patients.
\end{abstract}

\title{
Patient and methods
}

We report a neutropenic girl with a cervical rhabdomyosarcoma, treated with ifosfamide, vincristine, and actinomycin D (IVA), who developed repetitive periods of diarrhea, fever and abdominal complaints after each cytostatic treatment. Circulating levels of Intestinal Fatty Acid Binding Protein (I-FABP), a small cytosolic protein constitutively present in mature enterocytes, and inflammatory mediators were measured during five consecutive weeks.

\section{Results}

Strongly elevated plasma levels of I-FABP, representing enhanced enterocyte cell death, associated very well with periods of fever, severe intestinal complaints and peak values of IL-6, IL-8 and CRP after chemotherapy.

\section{Conclusion}

This case is the first to show that plasma I-FABP levels allow early detection of chemotherapy induced enterocyte cell death. 


\section{Chapter 3}

\section{IINTRODUCTION}

Mucositis is a well known adverse effect of chemotherapy. Next to oral mucositis, gastro-intestinal $(\mathrm{Gl})$ mucositis is increasingly recognized as chemotherapy associated toxicity. The consequences of Gl mucositis are diarrhea, fever, inflammation with pain and risk for bacteremia and sepsis ${ }^{1}$. A means of objectively and early monitoring of Gl mucositis is necessary, since the diagnosis is currently based on signs and symptoms ${ }^{2}$. Moreover, there is a need for tools to study the pathobiology of Gl mucositis. Enterocyte loss is considered to be an important aspect of the pathophysiology of Gl mucositis; therefore evidence of enterocyte cell death was monitored in the circulation. This case is the first to show an association of severe enterocyte damage with known complications of chemotherapy induced GI mucositis.

\section{REPORT OF A CASE}

A 2-year old neutropenic girl with cervical rhabdomyosarcoma was admitted to the Pediatric Intensive Care Unit for chemotherapy induced septicemia with diarrhea and fever $\left(>38.5^{\circ} \mathrm{C}\right)$. Chemotherapy consisted of ifosfamide, vincristine, and actinomycin D (IVA) once in three weeks. This treatment is known to cause severe infection in $26 \%$ of treatment episodes ${ }^{3}$. Before, the patient showed twelve episodes of bacteremia, defined as positive blood cultures, with diarrhea, abdominal complaints and fever during eleven cytostatic treatments, resulting in severe discomfort. The patient recovered rapidly after each episode of bacteremia.

The signs and symptoms of the patient (diarrhea, fever, abdominal complaints) are suggestive for $\mathrm{Gl}$ mucositis as source for bacteremia. Direct evidence for intestinal mucosal damage in these patients with inexplicable systemic infectious complications is nevertheless not readily available, although ultrasound and computed tomography can help to differentiate GI complications in neutropenic patients ${ }^{4,5}$.

Intestinal Fatty Acid Binding Protein (I-FABP) has been reported to be a useful plasma marker for early enterocyte cell death ${ }^{6}$. The small (14-15 kDa) cytosolic IFABP is present in mature enterocytes of small and large intestine and released as soon as the cell membrane integrity is compromised. I-FABP is not normally found in plasma, but is rapidly detectable following acute intestinal ischemic cell death ${ }^{6}$.

During five consecutive weeks clinical signs were recorded and EDTA plasma was sampled. Plasma I-FABP was determined, using a human I-FABP ELISA that selectively detects human I-FABP (standard: 20-5000 pg/ml), provided by HBT (Uden, the Netherlands). In order to asses the systemic 
inflammatory response, circulating C-Reactive Protein (CRP), Interleukin-6 (IL6) and Interleukin-8 (IL-8) were measured. CRP was routinely determined; IL-6 and IL-8 were measured using in house ELISAs.

The patient experienced a bacteremic period with fever and diarrhea following the first cytostatic treatment and fever and diarrhea following the second treatment in the study-period of five weeks (Figure 1). A blood culture was positive for coagulase negative staphylococci. Both cytostatic treatments involved severe discomfort: diarrhea, fever and abdominal complaints. Interestingly, plasma I-FABP values associated very well with periods of fever and severe intestinal complaints seven days after first cytostatic treatment, and three and ten days after the second treatment. Peak values of five to twenty-fold raised I-FABP levels preceded the intestinal complaints and fever by approximately 4-24 hours. Each rise of I-FABP was followed by a rapid decline. At time points of high I-FABP levels also peak values of CRP were found. Both IL-6 and IL-8 were increased at the moment of maximal rise in I-FABP levels. These data indicate that the mucosal cell damage, as assessed by enhanced I-FABP plasma levels, was accompanied by a systemic inflammatory response, a characteristic of $\mathrm{Gl}$ mucositis ${ }^{7}$.

CRP
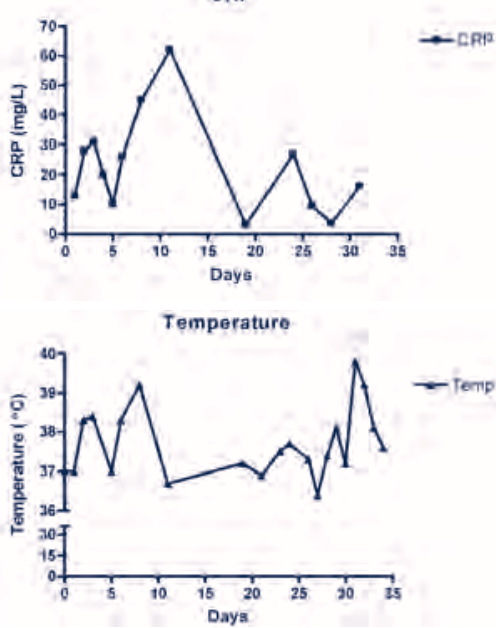

Figure 1: A 2-year old girl with rhabdomyosarcoma had three episodes of gastrointestinal mucositis and bacteremia following two cystostatic treatments (CT) consisting of ifosfamide, vincristine, and actinomycin D (IVA). Gastrointestinal mucostitis was accompanied by increased plasma intestinal fatty acid binding protein (I-FABP), reflecting enterocyte damage, raised C-reactive protein levels, and fever. Blood culture (BC) was positive for coagulase-negative staphylococci.
IL -6 and IL- 8
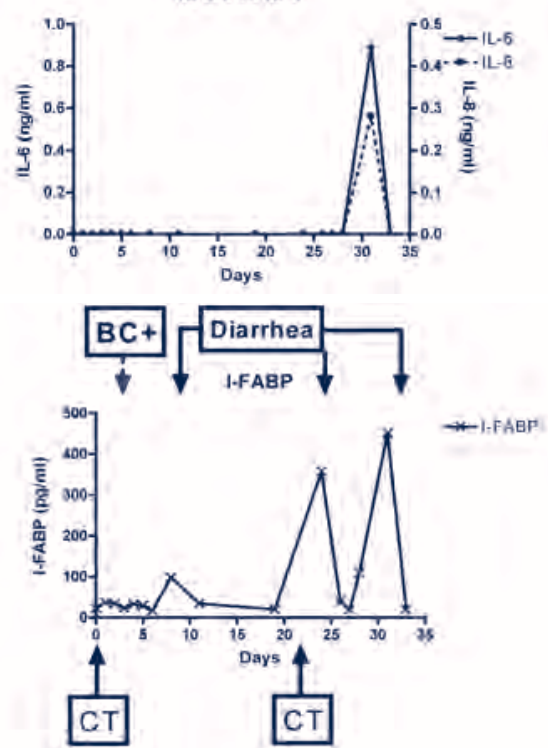

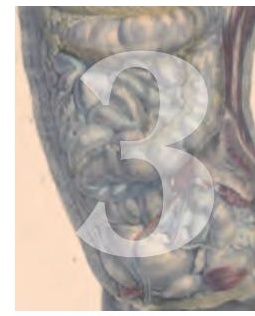




\section{Chapter 3}

\section{Discussion}

Fatty Acid Binding Proteins (FABPs) belong to a family of proteins that facilitate intracellular long-chain fatty acid transport. These relatively small (14-15kDa) cytosolic proteins are promising biomarkers for monitoring tissue damage in a number of organs: e.g. liver, kidney, intestine ${ }^{8}$. Next, FABPs are used as markers for colorectal cancer and renal cell carcinoma ${ }^{9,10}$. Potentially, measurement of the renal FABP-types $(\mathrm{H}$ - and L-FABP) could give information on renal toxicity in patients receiving ifosfamide. In the present study an ELISA that specifically quantifies I-FABP, as a specific marker for intestinal mucosal damage, has been used to assess $\mathrm{GI}$ mucositis.

So far, diagnosis of $\mathrm{Gl}$ mucositis is limited to scales scoring subjective symptoms and functional changes with ultrasonography and computed tomography as potentially helpful tools for the detection of neutropenic enterocolitis ${ }^{4,5}$. The data presented here show that plasma I-FABP represents a promising objective and early tool for the assessment of chemotherapy induced intestinal mucosal damage.

Following the first cytostatic treatment a significant increase in I-FABP levels was seen after seven days. This increase paralleled the inflammatory response, measured by raised plasma CRP and fever. The second cytostatic treatment was rapidly followed by an increase in circulating I-FABP, possibly indicative for a lack of sufficient intestinal mucosal repair after the first cytostatic treatment. After the second cytostatic treatment two I-FABP peaks were seen, of which the second was accompanied by a strong systemic inflammatory response, shown by increased IL-6, IL-8 and CRP levels and fever.

The diagnosis of $\mathrm{Gl}$ damage with I-FABP offers an important step towards a better insight in the pathophysiology of chemotherapy induced GI mucositis. Since I-FABP measurement can be performed rapidly and with high sensitivity, such measurements may offer new therapeutic options.

The mechanism of chemotherapy induced GI mucositis remains unclear. Recently, mucositis development has been divided into five phases: initiation, primary damage response, signal amplification, ulceration and healing ${ }^{11}$. At this stage we cannot discriminate between direct chemotherapy induced toxic enterocyte cell death and inflammation related enterocyte damage. However, the present data show that intestinal mucosal cell death in patients after chemotherapy is accompanied by a rapid increase of CRP, suggesting an involvement of an inflammatory process in the development of cell death. The cumulative release of I-FABP can be used to estimate the extent of damage, taking into account the renal FABP clearance rate. 
This case is the first to show that measurement of circulating I-FABP offers a valuable addition to the assessment of Gl mucositis. This is important, because intestinal mucosal damage provides a portal of entry for Gl microorganisms or initiates the systemic inflammatory response by itself, resulting in severe abdominal complaints with fever and sepsis.

\section{RefERENCES}

1. Peterson DE, Cariello A. Mucosal damage: a major risk factor for severe complications after cytotoxic therapy. Semin Oncol. 2004;31:35-44.

2. O'Brien SN, Blijlevens NM, Mahfouz $\mathrm{TH}$, et al. Infections in patients with hematological cancer: recent developments. Hematology (Am Soc Hematol Educ Program). 2003:438-72.

3. Stevens MC, Rey A, Bouvet N, et al. Treatment of nonmetastatic rhabdomyosarcoma in childhood and adolescence: third study of the International Society of Paediatric Oncology--SIOP Malignant Mesenchymal Tumor 89. J Clin Oncol. 2005;23:2618-28.

4. Cartoni C, Dragoni F, Micozzi A, et al. Neutropenic enterocolitis in patients with acute leukemia: prognostic significance of bowel wall thickening detected by ultrasonography. J Clin Oncol. 2001;19:756-61.

5. Kirkpatrick ID, Greenberg HM. Gastrointestinal complications in the neutropenic patient: characterization and differentiation with abdominal CT. Radiology. 2003;226:668-74.

6. Lieberman JM, Sacchettini J, Marks C, et al. Human intestinal fatty acid binding protein: report of an assay with studies in normal volunteers and intestinal ischemia. Surgery. 1997;121:335-42.

7. Blijlevens NM, Donnelly JP, DePauw BE. Inflammatory response to mucosal barrier injury after myeloablative therapy in allogeneic stem cell transplant recipients. Bone Marrow Transplant. 2005;36:703-7.

8. Pelsers MM, Hermens WT, Glatz JF. Fatty acid-binding proteins as plasma markers of tissue injury. Clin Chim Acta. 2005;352:15-35.

9. Yamazaki T, Kanda T, Sakai Y, et al. Liver fatty acid-binding protein is a new prognostic factor for hepatic resection of colorectal cancer metastases. J Surg Oncol. 1999;72:83-7.

10. Seliger B, Lichtenfels R, Atkins D, et al. Identification of fatty acid binding proteins as markers associated with the initiation and/or progression of renal cell carcinoma. Proteomics. 2005;5:2631-40.

11. Sonis ST. The pathobiology of mucositis. Nat Rev Cancer. 2004;4:277-84. 


\subsection{Loss of enterocyte mass is accompanied by diminished turnover of enterocytes after myeloablative therapy in haematopoietic stem cell transplant recipients}

Joep P. M. Derikx, Nicole M. A. Blijlevens, J. Peter Donnelly, Hiroshi Fujii, Tatsuo Kanda, Annemarie A. van Bijnen, Erik Heineman, Wim A. Buurman Annals of Oncology, (2009) 20: 337-42. 


\section{Summary}

\section{Background}

Intestinal mucosal barrier injury $(\mathrm{MBI})$, resulting from myeloablative conditioning for haematopoietic stem cell transplantation (HSCT) is an important cause of morbidity. Despite its frequency, recognition presents a challenge, while the aetiology needs still to be unravelled. The relationship between enterocyte mass and enterocyte loss was explored by examining citrulline serum levels and by assessing circulating I-FABP and I-BABP, proteins released by dying mature enterocytes.

\section{Patients and methods}

34 adult patients with haematological malignancy received allogeneic HSCT (HSCT day 0) 12 days after being given idarubicin, cyclophosphamide and total body irradiation as myeloablative conditioning, a regimen known to induce oral and intestinal MBI. Serum levels of citrulline, I-FABP and I-BABP were measured on HSCT days $-12,-6,0,+7,+14,+21$.

\section{Results}

Myeloablative conditioning resulted in a significant decrease in serum citrulline with the nadir on HSCT day +7 ; thereafter levels rose gradually. Simultaneously, a significant decrease in I-FABP and I-BABP levels occurred from the day of transplant until day +14 .

\section{Conclusions}

Simultaneous reduction and subsequent increase of citrulline, and I-FABP, IBABP levels following cytotoxic treatment show that enterocyte mass corresponds to lower rate of dying enterocytes, indicating reduced turnover of enterocytes. Assessment of enterocyte turnover and mass offers opportunities for evaluation of new MBI therapies. 


\section{Chapter 3}

\section{INTRODUCTION}

Mucosal Barrier Injury (MBI) of the digestive tract is an inevitable and frequent side-effect of the intensive myeloablative conditioning employed to prepare for haematopoietic stem cell transplantation (HSCT). MBI presents with oral mucositis and corresponding gastro-intestinal signs and symptoms of mucositis, such as abdominal pain, diarrhoea, vomiting and weight loss ${ }^{1,2}$. MBI together with profound neutropenia predispose patients to local invasion by microorganisms and, subsequently, to systemic life-threatening infections ${ }^{2,3}$.

MBI has been attributed to the high physiological proliferation and turnover of intestinal epithelial cells, which are highly susceptible to cytotoxic therapy ${ }^{2,4,6}$. Evidence for the loss of small bowel epithelial cell mass has been provided by declined serum levels of citrulline in HSCT recipients suffering from severe oral and gastrointestinal mucositis following intensive myeloablative therapy ${ }^{7,8}$. The levels of circulating citrulline, an amino acid not incorporated into proteins, reflect functional enterocyte mass. Differentiated small intestinal enterocytes specifically produce citrulline from glutamine and are responsible for the major part of the total amount of serum citrulline ${ }^{9,10}$.

The origin of the reduced functional intestinal epithelial cell mass is hitherto unclarified. Both an increased cell death of enterocytes and a reduced turnover might explain the reduced functional enterocyte mass. Measurement of endogenous cytosolic enterocyte proteins, such as Fatty Acid Binding Proteins (FABP) have been shown to be useful to estimate enterocyte loss, e.g. after episodes of acute intestinal ischemia and inflammation, including necrotizing enterocolitis ${ }^{11,13}$. FABP comprise a class of low molecular weight (14-15 kDa) cytosolic proteins found in high concentrations in tissues involved in the uptake and consumption of fatty acids ${ }^{14}$. Intestinal Fatty Acid Binding Protein (I-FABP) is primarily limited to mature enterocytes of small and large intestine. It circulates in low amounts in the blood stream of healthy individuals 11. Ileal-Bile Acid Binding Protein (I-BABP) is exclusively present in mature enterocytes of the jejunum and ileum ${ }^{15}, 16$. Liver-FABP (L-FABP) is localized in small amounts in the mature enterocytes of the small and large intestine but in abundance in the liver ${ }^{14}$.

The expression of L-FABP and I-FABP mRNA and proteins was barely affected during the phase of crypt and villous damage in rats treated with the cytostatic drug methotrexate (MTX) unlike what was found for other enterocyte markers, including glucose transporter 5 (GLUT5), carbamoylphosphatate synthase (CPS), sucrase-isomaltase (SI), sodium glucose co-transporter 1 (SGLT1) and lactase. This was underscored by immunohistochemistry ${ }^{17}$. 
In the present study circulating levels of FABP were determined longitudinally along with citrulline serum levels in a cohort of HSCT recipients suffering from severe mucositis after intensive myeloablative conditioning in order to relate the known reduced functional enterocyte mass with enhanced cell death or reduced turnover of enterocytes.

\section{Patients AND methods}

\section{Patients}

Between July 1999 and July 2002, 34 adult patients admitted to the department of Haematology, Radboud University Nijmegen Medical Centre, received a Tcell depleted sibling HSCT on HSCT day 0 to treat haematological malignancy and consented to participate in the study ${ }^{7,18}$. The preparative myeloablative conditioning regimen consisted of idarubicin starting 12 days before transplant (HSCT day -12), followed by cyclophosphamide on HSCT days -6 and -5 and a total of 9 Gray total body irradiation on HSCT days -2 and -1 . All patients received cyclosporin and the same anti-infective prophylaxis as well as empirical antibiotic therapy when fever occurred. The local ethics committee approved the study.

\section{Citrulline assay}

Serum had been collected on HSCT days $-12,-6,0,+7,+14$ and +21 . In practice, some samples were not taken. The serum was stored in aliquots at $80^{\circ} \mathrm{C}$ until further analysis. Citrulline concentrations were measured $(\mu \mathrm{M})$ by a standard procedure for determining amino acids ${ }^{7}$.

\section{I-FABP assay}

Serum I-FABP was determined using a highly specific commercially available enzyme-linked immunosorbent assays (ELISA) that selectively detects human IFABP (standard: 20-5,000 pg/ml) (provided by Hycult Biotechnology, Uden, the Netherlands).

\section{I-BABP assay}

The I-15P expression vector pET/Hu I-15P, which contains cDNA encoding human I-BABP, was kindly donated by Dr. Fujii 15, 16. Prokaryotic protein expression was achieved by transformation of pET/Hu l-15P into BL21 cells. After harvesting and lysing of the cells, the recombinant human I-BABP was purified with an ion-exchanger (DE-52, Whatman, Kent, UK) and gel filtration (Superdex-75 column, Pharmacia GE Healthcare, Diegem, Belgium). The 


\section{Chapter 3}

antiserum against recombinant human I-BABP was obtained by immunizing of New-Zealand white rabbits with I-BABP and adjuvant carried out under a protocol approved by the Institutional Animal Care Committee of the University of Maastricht. The rabbit antiserum was purified by affinity chromatography on protein A-sepharose.

The following ELISA was developed to measure human I-BABP. Anti-I-BABP IgC was coated at a concentration of $2.5 \mu \mathrm{g} / \mathrm{ml}$ in PBS 1 hour at $37^{\circ} \mathrm{C}$ on a 96 -well plate (Immuno-Maxisorp; Nunc, Roskilde, Denmark). Free sites were blocked by $1 \mathrm{~h}$ of incubation with $1 \%$ BSA in PBS at room temperature. Test samples as well as human recombinant I-BABP, used as standard, were incubated for $1 \mathrm{~h}$ at room temperature. Then biotinylated anti-I-BABP IgG was added as the detection antibody for $1 \mathrm{~h}$ at room temperature. HRP-streptavidin conjugate (Zymed Laboratories, Inc., San Francisco, CA) was used to develop the color reaction in combination with 3,3,5,5-tetramethylbenzidine (Kirkegaard \& Perry Laboratories, Gaithersburg, MD) and $\mathrm{H} 2 \mathrm{SO} 4$. Color intensity was measured by determining the absorbance at $450 \mathrm{~nm}$ using a micro-ELISA reader. The detection range was $0.32-5 \mathrm{ng} / \mathrm{ml}$. The specificity of the rabbit anti-human IBABP was tested by incubating human recombinant I-FABP, L-FABP and lysed human ileum and colon together with the standard human recombinant I-BABP. The anti-I-BABP specifically reacted with human I-BABP, human ileum, but not with human colon, I-FABP or L-FABP.

\section{Statistical analysis}

Statistical analysis was performed using Prism 4.0 for Windows (GraphPad Software Inc. San Diego, CA). Concentrations were presented as mean \pm standard error (SEM). Normality of all data obtained was verified by Kolmogorov-Smirnov test. Distribution of serum citrulline and FABP concentrations did not pass the normality test on HSCT day +7 for citrulline and I-BABP and on HSCT day $0,+7$ and +14 for I-FABP. Therefore, Friedman two-way analysis of variance by ranks was used to analyze changes between HSCT days in serum citrulline and FABP levels. A Wilcoxon matched pairs test was used to calculate significant differences of serum citrulline and FABP with respect to baseline (HSCT day -12). Correlations were calculated using Spearman correlation coefficient. A p-value below 0.05 was considered to be statistically significant. 


\section{ResUlts}

\section{Patient characteristics}

Of the original 34 patients 5 patients were excluded from this study, because blood was collected on less than 3 study days. The remaining 29 patients (11F:18M) had a mean age of 49 years (range: $25-65$ years). Nine patients had acute myeloid leukaemia, 6 had non-Hodgkin lymphoma, 5 had acute lymphoblastic leukaemia, 5 had myelodysplastic syndrome, 2 had chronic myeloid leukaemia, and one patient each had myeloproliferative disease, chronic lymphocytic leukaemia and myelofibrosis. All had received a T-cell depleted sibling HSCT to treat their haematological malignancy

\section{Transplant-related complications}

All patients suffered from severe oral and intestinal mucosits ${ }^{18}$. All except 1 patient were treated empirically for fever during neutropenia with a cephalosporin. 9 patients had bacteraemia due to oral viridans streptococci occurring between HSCT days +1 and +8 . Acinetobacter baumanii was recovered from a single aerobic blood culture from one patient on day +1 after transplant but no other Gram negative bacilli were recovered from blood cultures or surveillance specimens of any other patient during the study period.

There were 8 serious adverse events resulting in death. 4 patients died because of an infectious event: one on HSCT day +9 because of sepsis syndrome, another on HSCT day +12 because of sepsis syndrome after Streptococcus mitis bacteraemia, one on HSCT +13 due to invasive Candida infection and a fourth on HSCT day +21 because of invasive pulmonary aspergillosis. Another 4 patients died because of a non-infectious event due to multi-organ failure on HSCT days +13 and +43 respectively and one because of grade IV acute GvHD on HSCT day +55 and the other due to cerebral haemorrhage after primary graft rejection on HSCT day +56 . Although the mortality rate is high, it is not markedly higher than what has been reported ${ }^{19}$. This particular intensive preparative myeloablative conditioning regimen consisted of idarubicin, cyclophosphamide and total body irradiation and was used to counterbalance the increased graft rejection and increased relapse rate associated with the T-cell depletion used at that time.

\section{Serum citrulline concentrations after myeloablative conditioning regimen for HSCT}

The mean (SEM) initial citrulline serum concentration on HSCT day -12 was 31.6 (1.6) $\mu \mathrm{M}$, which was comparable to baseline values in previous study ${ }^{7}$. 


\section{Chapter 3}

Following HSCT, a significant decrease in serum citrulline concentrations was observed (Friedman test: $p<0.0001$ ) with the nadir observed on HSCT day +7 (10.5 (1.5) $\mu \mathrm{M}$, Figure 1, see Figure 2a for 7 individual patients). At the last study day (HSCT day +21), the mean serum citrulline value (15.1 (1.4) $\mu \mathrm{M})$ had increased, but was still significantly lower than baseline $(p=0.0005)$. Since serum citrulline concentration had been shown to be a reliable marker of functional enterocyte mass, our data indicate that myeloablative therapy, accompanied by symptoms and signs of severe mucosal damage, results in clear transient reduction in the small intestinal epithelial cell mass ${ }^{7}$.

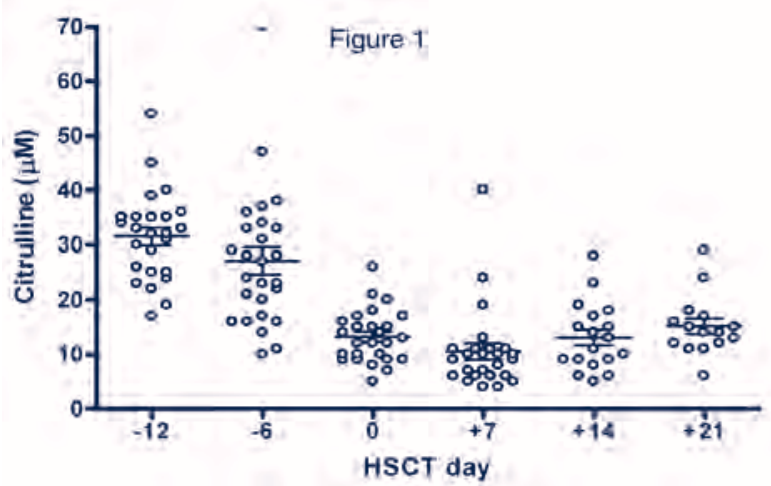

Figure 1: Serum citrulline concentrations after myeloablative conditioning regimen for HSCT. Serum citrulline concentrations declined significantly at HSCT day -6 and remain decreased until HSCT day +21 , indicating rapid and continuous loss of small bowel enterocyte mass following intensive myeloablative therapy.

\section{Serum I-FABP and I-BABP concentrations after myeloablative conditioning regimen for $\mathrm{HSCT}$}

In the untreated situation, substantial variability in I-FABP serum concentrations was noted, ranging from 20 to $336 \mathrm{pg} / \mathrm{ml}$ (Figure 3a, see Figure $2 \mathrm{~b}$ for 7 individual patients). Following myeloablative conditioning regimen for HSCT, the Friedman test indicated a significant decrease in serum I-FABP values during the study period $(p=0.0009)$. Serum I-FABP levels remained unchanged at HSCT day $-6(67.9(13.6) \mathrm{pg} / \mathrm{ml}, \mathrm{p}=0.416)$. From HSCT day 0 a significant decrease of I-FABP levels (27.6 (4.2) pg/ml, p=0.001) compared to day -12 was found, which proceeded until HSCT day +14 . The decrease in serum I-FABP concentration between HSCT day -6 and 0 occurred in all but one patient. The lowest mean I-FABP concentration measured was found on HSCT day +7 (21.1 (1.1) $\mathrm{pg} / \mathrm{ml}$ ), similar to the nadir of serum citrulline values. However, in contrast 

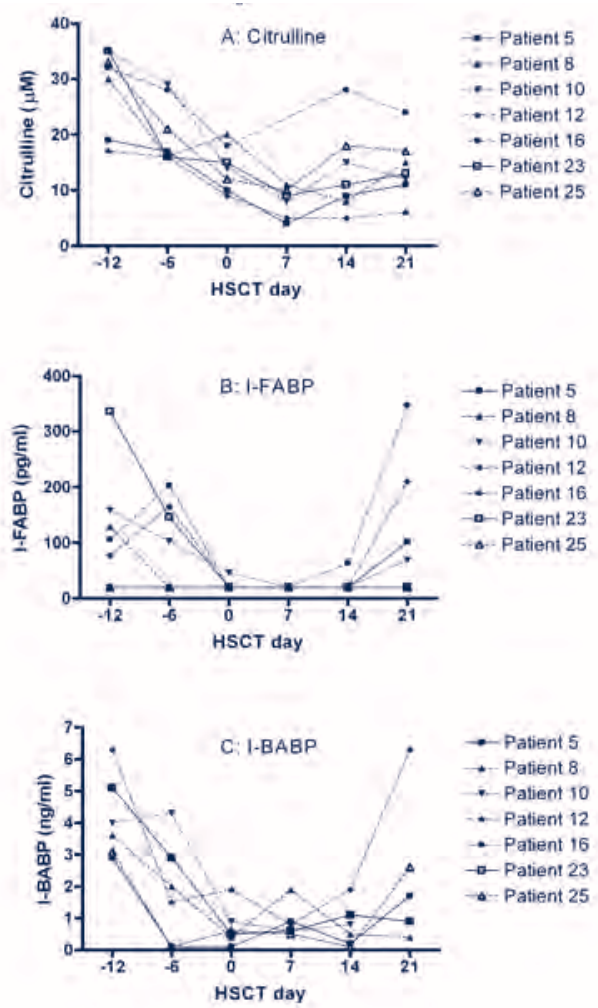

Figure 2: Serum citrulline, I-FABP and I-BABP concentrations for 7 individual patients after myeloablative conditioning regimen for HSCT. Serum citrulline (a), I-FABP (b) and I-BABP (c) concentrations show that almost all patients follow the same pattern.

to serum citrulline levels, I-FABP returned to baseline levels on HSCT day +21 (86.3 (28.6) pg/ml, p=0.922).

Similar to the I-FABP levels, also for I-BABP serum concentration substantial variation was present before treatment with a myeloablative conditioning regimen for HSCT, ranging from 0.6 to $8.5 \mathrm{ng} / \mathrm{ml}$ (Figure $3 \mathrm{~b}$, see Figure 2c for 7 individual patients). Following preparative regimen, the Friedman test indicated significant reduction in serum I-BABP levels during the study period $(p<0.0001)$. Serum I-BABP levels showed from HSCT day -6 onwards a significant decrease $(2.1(0.6) \mathrm{ng} / \mathrm{ml}, \mathrm{p}=0.004)$ compared to HSCT day -12 . This diminution continued until HSCT day +14 . The decrease in serum I-BABP concentration between HSCT day -12 and -6 occurred in 26 out of 29 patients. The nadir mean I-BABP concentration was found on HSCT day 0 (0.8 $(0.1) \mathrm{ng} / \mathrm{ml})$. In accordance with the normalisation of serum I-FABP levels on 
HSCT day +21 , a return to baseline levels of serum I-BABP on HSCT day +21 was found (3.7 (1.6) ng/ml, p=0.470 vs HSCT day -12). Interestingly, all serum I-FABP levels correlated significantly with serum I-BABP levels (Spearman $r=0.436, p<0.0001)$.
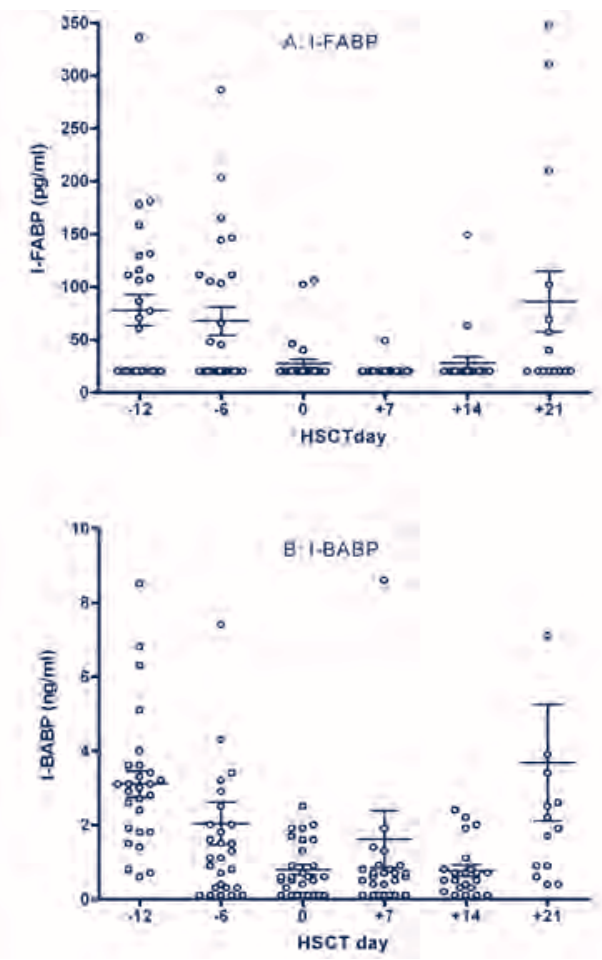

Figure 3: Serum I-FABP and I-BABP concentrations after myeloablative conditioning regimen for HSCT. Serum I-FABP (a) and I-BABP (b) concentrations decreased significantly at HSCT day 0 and returned to baseline levels at HSCT day +21 , indicating loss of turnover of intestinal epithelial cells followed by a recovery after 3 weeks in patients undergoing intensive myeloablative therapy.

\section{Ratio of serum I-FABP:citrulline and I-BABP:citrulline after myeloablative conditioning regimen for HSCT}

In order to study whether the functional cell mass is directly related to the loss of villous enterocytes, the ratio of serum values of I-FABP and citrulline was determined at each time point. At baseline the mean ratio I-FABP:citrulline was 27.6 (4.8) (Figure 4a). The ratio remained unchanged during the complete study period (Friedman test: $p=0.600$ ), indicating that a lower mass of functional small 
bowel enterocytes is accompanied by loss of enterocytes. Only on HSCT day +21 a higher mean ratio was found (66.5 (24.2)) compared to all preceding study days, caused by a relative elevation of I-FABP rather than citrulline levels. However, this increased ratio was not significant $(p=0.424)$ compared to HSCT day -12 .

The specificity of I-BABP for mature enterocytes of jejunum and ileum allows us to relate the loss of enterocytes of the jejunum and ileum with functional cell mass. Before treatment the mean ratio I-BABP:citrulline was 11.6 (1.9) (Figure $4 \mathrm{~b}$ ). The ratio remained unaltered during the study period (Friedman test: $p=0.460$ ), indicating that the mass of small bowel enterocytes is related with the loss of jejunal and ileal enterocytes.
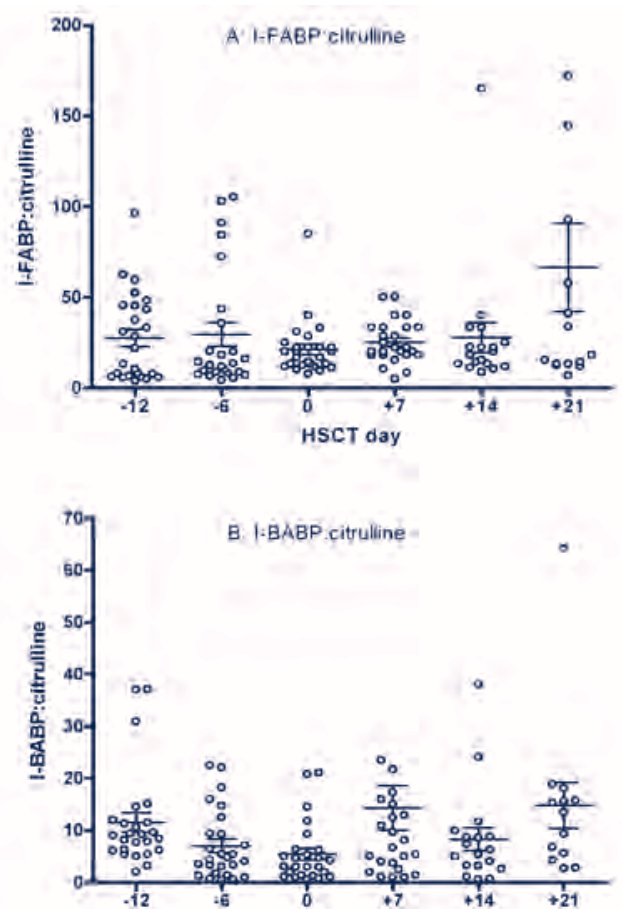

Figure 4: Ratio of serum I-FABP:citrulline and I-BABP:citrulline after myeloablative conditioning regimen for HSCT. Ratio of serum I-FABP:citrulline (a) and I-BABP:citrulline (b) remains unaltered in patients with intensive myeloablative therapy, indicating that the loss of small bowel enterocyte mass correlates with a deficit of turnover of intestinal epithelial cells. 


\section{Discussion}

Human histological studies on intestinal mucositis are nearly impossible due to the severity of mucositis and increased bleeding tendency. Therefore, circulating levels of citrulline and enterocyte cytosolic proteins I-FABP and I$\mathrm{BABP}$ are investigated longitudinally in a cohort of patients receiving the same intensive myeloablative conditioning for HSCT. A substantial variation in circulating levels of both FABP before treatment is observed, which is comparable with data of healthy individuals ${ }^{20}$. The expression of both I-FABP and I-BABP is limited to differentiated enterocytes present on the upper one third part of the villus in the small intestine ${ }^{11,16}$. These FABP-containing cells are known to detach from the villus or die at the end of their lifespan ${ }^{21}$. Taking into consideration the very short half-life of these proteins (approximately 11 minutes), we consider that the baseline I-FABP and I-BABP circulating levels represent the continuous release of $\mathrm{FABP}$ originating from mature detaching or dying enterocytes. Some of the baseline FABP (and citrulline) serum levels might also relate to previous chemotherapy. Since the main loss of enterocytes in the healthy intestine consists of senescent enterocytes which detach into the gut lumen at the end of their lifespan, the steady state circulating levels of FABP represent the loss of enterocytes, and thus also the production of enterocytes or in other words the turnover of enterocytes.

Interestingly, a minor increase in the amount of serum FABP was found only in three patients after initiation of the myeloablative conditioning regimen. This indicates that no direct (small) bowel epithelial cell damage occurred during this preparative regimen for HSCT at this time. However, following the preparative regimen levels of both I-BABP and I-FABP declined in virtually every case. These data show that less cytosolic content of enterocytes is released into the circulation than under normal, healthy circumstances, indicating a decreased rate of dying enterocytes. This, in turn, suggests a reduced turnover of enterocytes after myeloablative conditioning.

This is the first study to report on specific changes in I-BABP levels, representing specific jejunal and ileal enterocyte loss, using a newly developed ELISA for measurement of serum and plasma I-BABP. Our study shows a very rapid decrease in I-BABP circulating levels, a representative of diminished small intestinal turnover of enterocytes following onset of therapy. This occurred one week earlier than the marked reduction in I-FABP which is present in enterocytes of the whole intestine. This is consistent with the enhanced susceptibility of small bowel enterocytes to myeloablative regimen and is clearly shown by the rapid alteration of the circulating marker I-BABP. 
Significantly lower serum citrulline concentrations, an indicator for the functional small bowel epithelial cell mass, occur two weeks after the start of myeloablative therapy lagging one week behind diminished I-BABP levels, and correspond with the decreased I-FABP circulating levels. Importantly, it took about three weeks before levels of circulating FABP increased towards normal values, indicating an elevated turnover of enterocytes, which most likely reflects recovery by proliferation. Similarly, rising serum citrulline concentrations indicate an initiation of the recovery of functional enterocyte mass on HSCT day +21 . The simultaneous alterations of serum levels of I-BABP, I-FABP (representing loss of cells present in the jejunum-ileum and total intestine, respectively), and serum concentrations of citrulline (representing total small intestinal enterocyte mass) indicate that turnover and mass of intestinal epithelium are directly related. This is further supported by the ratios of serum I-FABP:citrulline and I-BABP:citrulline, which clearly indicate that the turnover of (small) intestinal epithelial cells corresponds to the small bowel epithelial cell mass throughout the entire study period.

From these data, we conclude that the reported reduction in enterocyte mass is directly related to a reduced turnover of enterocytes. This lower turnover could be caused by a reduced number of enterocyte stem cells, which is in line with the kinetics of injured gut epithelium after radiotherapy and chemotherapy ${ }^{4-6,22}$. Human duodenal biopsies of patients receiving nonintensive chemotherapy showed induction of apoptosis in the intestinal crypts, using TUNEL technique and electron microscopy, which occurred before hypoplastic villous atrophy, loss of enterocyte cell height and decreased mitotic count ${ }^{4}$. Presumably, apoptosis reduced the net functional stem cell population. The authors concluded that the chemotherapy-induced apoptosis of intestinal crypts in patients was not accompanied with mucosal destruction, but rather with morphometric reduction in crypt size and proliferation ${ }^{4}$. Also the recently defined five-phase process of the pathobiology of mucositis described that radiation or chemotherapy leads to direct and indirect injury to epithelial stem cells, resulting in a loss of renewal capacity in the early phase of 'primary damage response and signal amplification' ${ }^{6}$. Rats treated with methotrexate (MTX) showed a similar inhibition of epithelial proliferation, decreased crypt depth, and crypt cell apoptosis in the small intestine ${ }^{5}$. In line with these data, we showed no increase of circulating I-FABP and I-BABP levels, indicating no (small) intestinal mucosal cell damage. Our data are supported by the work of Vigneulle et al. in nonhuman primates who show that following radiationinduced intestinal injury an ablation of intestinal epithelial stem cells leads to a compensatory reduction in villus length (loss of total villous cell mass), which 


\section{Chapter 3}

our work indicates to be accompanied by a reduction in loss of senescent cells (cell turnover) ${ }^{22}$. The compensatory regeneration of stem cells and villus epithelial cells took more than five weeks to result in a complete recovery, which is in line with our data on continuous low levels of circulating citrulline after myeloablative conditioning ${ }^{22}$. Our data indicate that the loss of senescent cells prevails the complete recovery period.

Intestinal mucositis is a well known adverse effect of myeloablative therapy, and a risk factor for mortality and relapse in patients undergoing autologous stem cell transplantation, probably due to necessary reductions in treatment intensity and sometimes cessation of treatment ${ }^{23}$. New therapies to treat this toxicity are being developed, which are aimed at protection and enlargement of the enterocyte mass ${ }^{24,25}$. Combined measurement of the turnover (serum FABP level) and mass (serum citrulline levels) of enterocytes offers the new modality to evaluate such novel treatments in patients.

\section{REFERENCES}

1. Peterson DE, Cariello A. Mucosal damage: a major risk factor for severe complications after cytotoxic therapy. Semin Oncol 2004; 31: 35-44.

2. Niscola P, Romani C, Cupelli L et al. Mucositis in patients with hematologic malignancies: an overview. Haematologica 2007; 92: 222-231.

3. Blijlevens NM. Implications of treatment-induced mucosal barrier injury. Curr Opin Oncol 2005; 17: 605-610.

4. Keefe DM, Brealey J, Goland GJ et al. Chemotherapy for cancer causes apoptosis that precedes hypoplasia in crypts of the small intestine in humans. Gut 2000; 47: 632-637.

5. Verburg M, Renes IB, Meijer HP et al. Selective sparing of goblet cells and paneth cells in the intestine of methotrexate-treated rats. Am J Physiol Gastrointest Liver Physiol 2000; 279: G1037-1047.

6. Sonis ST. The pathobiology of mucositis. Nat Rev Cancer 2004; 4: 277-284.

7. Blijlevens NM, Lutgens LC, Schattenberg AV et al. Citrulline: a potentially simple quantitative marker of intestinal epithelial damage following myeloablative therapy. Bone Marrow Transplant 2004; 34: 193-196.

8. Lutgens LC, Blijlevens NM, Deutz NE et al. Monitoring myeloablative therapy-induced small bowel toxicity by serum citrulline concentration: a comparison with sugar permeability tests. Cancer 2005; 103: 191-199.

9. Crenn P, Coudray-Lucas C, Thuillier F et al. Postabsorptive plasma citrulline concentration is a marker of absorptive enterocyte mass and intestinal failure in humans. Gastroenterology 2000; 119: 1496-1505. 
10. van de Poll MC, Ligthart-Melis GC, Boelens PG et al. Intestinal and hepatic metabolism of glutamine and citrulline in humans. J Physiol 2007; 581: 819-827.

11. Lieberman JM, Sacchettini J, Marks $C$ et al. Human intestinal fatty acid binding protein: report of an assay with studies in normal volunteers and intestinal ischemia. Surgery 1997; 121: 335-342.

12. Kanda T, Fujii H, Tani T et al. Intestinal fatty acid-binding protein is a useful diagnostic marker for mesenteric infarction in humans. Gastroenterology 1996; 110: 339-343.

13. Derikx JP, Evennett NJ, Degraeuwe PL et al. Urine based detection of intestinal mucosal cell damage in neonates with suspected necrotising enterocolitis. Gut 2007; 56: 1473-1475.

14. Pelsers MM, Hermens WT, Glatz JF. Fatty acid-binding proteins as plasma markers of tissue injury. Clin Chim Acta 2005; 352: 15-35.

15. Fujita M, Fujii H, Kanda T et al. Molecular cloning, expression, and characterization of a human intestinal 15-kDa protein. Eur J Biochem 1995; 233: 406-413.

16. Watanabe K, Hoshi N, Tsuura Y et al. Immunohistochemical distribution of intestinal 15 kDa protein in human tissues. Arch Histol Cytol 1995; 58: 303-306.

17. Verburg M, Renes IB, Van Nispen DJ et al. Specific responses in rat small intestinal epithelial mRNA expression and protein levels during chemotherapeutic damage and regeneration. J Histochem Cytochem 2002; 50: 1525-1536.

18. Blijlevens NM, Donnelly JP, Naber AH et al. A randomised, double-blinded, placebocontrolled, pilot study of parenteral glutamine for allogeneic stem cell transplant patients. Support Care Cancer 2005; 13: 790-796.

19. Schaap N, Schattenberg A, Bar B, et al. Outcome of transplantation for standard-risk leukaemia with grafts depleted of lymphocytes after conditioning with an intensified regimen. Br J Haematol 1997; 98: 750-759.

20. Derikx JP, Poeze M, van Bijnen AA et al. Evidence for intestinal and liver epithelial cell injury in the early phase of sepsis. Shock 2007; 28: 544-548.

21. Bullen TF, Forrest $S$, Campbell $F$ et al. Characterization of epithelial cell shedding from human small intestine. Lab Invest 2006; 86: 1052-1063.

22. Vigneulle RM, Rao S, Fasano A et al. Structural and functional alterations of the gastrointestinal tract following radiation-induced injury in the rhesus monkey. Dig Dis Sci 2002; 47: 1480-1491.

23. Fanning SR, Rybicki L, Kalaycio M et al. Severe mucositis is associated with reduced survival after autologous stem cell transplantation for lymphoid malignancies. $\mathrm{Br} J$ Haematol 2006; 135: 374-381.

24. Blijlevens NM, Sonis ST. Palifermin (recombinant keratinocyte growth factor-1): a pleiotropic growth factor with multiple biological activities in preventing chemotherapy- and radiotherapy-induced mucositis. Ann Oncol 2007; 18: 817-826.

25. Keefe DM. Intestinal mucositis: mechanisms and management. Curr Opin Oncol 2007; 19: 323-327. 


\subsection{Does gut damage occur around closure of gastroschisis with bladder herniation?}

Joep P. M. Derikx, Jolanda M. L. G Gehlen, Nicholas J. Evennett, Mark A. H. B. M. van der Hoeven, Annemarie A. van Bijnen, Wim A. Buurman, Erik Heineman

Submitted. 


\begin{abstract}
Reduction of organs during gastroschisis repair is often accompanied by elevated abdominal pressure, subsequent splanchnic mucosal hypoperfusion and intestinal epithelial cell damage, leading to complications as abdominal compartment syndrome, necrotizing enterocolitis and ventilator dependence. Furthermore, postoperative initiation and increase of enteral nutrition are at present guided by observation and physical examination, which have limitations. We report a patient with gastroschisis with rarely observed association of a bladder evisceration. The defect was effectively primarily closed guided by measurement of abdominal pressure by an indwelling urinary catheter. Intestinal mucosal cellular condition was monitored by assessment of urinary I-FABP. The reduction of organs did neither lead to increased I-FABP levels, nor to elevated intravesical pressure. Oral feeding could be started and increased successfully postoperatively. The level of urinary I-FABP was associated with intestinal functional recovery.

This case shows that intestinal mucosal cell damage, which is a major source of complications during and after gastroschisis repair, did nor occur during primary closure of the abdominal wall after gastroschisis, neither during the start and increase of enteral feeding.
\end{abstract}




\section{Chapter 3}

\section{INTRODUCTION}

With an incidence of approximately 1:9000 live births, gastroschisis is a relatively common congenital disorder. ${ }^{1}$ Diagnosis and treatment have become well standardized and good survival data are reported for the majority of patients. However, postoperative complications still occur, including necrotizing enterocolitis (NEC), bowel infarction, and ventilator dependence. ${ }^{1}$ It has been suggested that most of these complications after reduction of organs result from increased intra-abdominal pressure, leading to abdominal compartment syndrome, which causes inadequate perfusion and subsequent damage of the highly susceptible luminal surface of the intestinal mucosa. ${ }^{1,2}$ Gastric tonometric assessment of gastro-intestinal mucosal hypoperfusion and intravesical bladder monitoring, an indirect assessment of intra-abdominal pressure, are invasive tools that have been reported to allow estimation of intraabdominal pressure and to predict the safety of primary and secondary closures. ${ }^{2,3}$ Furthermore, postoperative initiation and increase of enteral nutrition are at present guided by observation and physical examination, which have their limitations. Therefore, there is a clinical need to study the intestinal mucosal condition shortly after closure of the abdominal wall as well as the condition of the intestinal mucosa during start and increase of enteral nutrition. Recently we showed that urine based detection of intestinal mucosal cell damage in neonates is possible with the use of Intestinal Fatty Acid Binding Protein (I-FABP). ${ }^{4}$ The small cytosolic I-FABP is a protein specifically present in mature enterocytes of small and large intestine and released as soon as the cell membrane integrity is compromised after episodes of acute intestinal ischemia and inflammation. ${ }^{4,5}$ Due to its small molecular weight (14-15 kDa), I-FABP present in the systemic circulation passes the glomerular filter (fractional renal excretion: $28 \%$, half-life time: 11 minutes ${ }^{6}$ ) and can readily be detected in urine. I-FABP is not expressed in the urinary tract mucosa. In 17 prematures with gastrointestinal symptoms suspicious for NEC urinary I-FABP proved to be a simple, non-invasive marker enabling early and specific detection of intestinal mucosal cell damage, a hallmark of NEC. ${ }^{4}$ Therefore, neonates who ultimately developed NEC could be distinguished in an early stage from the neonates who finally developed other neonatal diseases that present with abdominal signs, such as sepsis and constipation. ${ }^{4}$ In conclusion, urinary values of I-FABP provide actual information on intestinal mucosal cell damage. This new noninvasive diagnostic tool was used to monitor the intestinal mucosal condition in a patient who presented with gastroschisis. 


\section{Case report}

A 3060-g female infant was born at 38 1/7 weeks of gestation by caesarean section after antenatal diagnosis of gastroschisis. The parents were consanguine and from Turkish origin. At inspection, the gastroschisis surprisingly included evisceration of stomach, colon, small bowel, bladder, uterus, Fallopian tubes and ovaries (Fig. 1). At 3 hours after birth, the baby underwent surgery. Intraoperative measurement of abdominal pressure was performed with an indwelling urinary catheter to monitor the development of increased intraabdominal pressure. ${ }^{3}$ During surgery, malrotation was found accompanied by a very small descending colon, without signs of atresia. The defect of the abdominal wall was $3 \mathrm{~cm}$. Reduction of the organs could easily be performed, after manual stretching of the abdominal wall, where after the defect was closed primarily. The maximal intravesical pressure during the operation was 18 $\mathrm{mm} \mathrm{Hg}$. The intravesical pressure one day after surgery was decreased to 13 $\mathrm{mm} \mathrm{Hg}$. Ventilatory support was abandoned within 24 hours after surgery. Four days after surgery, minimal enteral feeding was started without problems.

The baby made excellent clinical progress during the first three weeks and was transported from the Neonatal Intensive Care Unit (NICU) to the Medium Care Unit. Enteral feeding was introduced progressively.

At day 21 the neonate developed fever with thrombocytopenia. Blood cultures were positive for Enterobacter cloacae. An abdominal ultrasound was performed in search for the source of the sepsis. While no gut abnormalities or abnormal fluid collections were noted, significant hepatosplenomegaly and distension of the gallbladder full of sludge were observed. The differential diagnosis included among others secondary hemophagocytic lymphioblastocytosis, because of the sepsis, the Turkish ethnicity, the thrombocytopenia and hepatosplenomegaly. However, the definite diagnosis could not be established because the infant died 36 days after surgery, despite receiving multiple aggressive antibiotic regimes. Consent for autopsy was not obtained. 


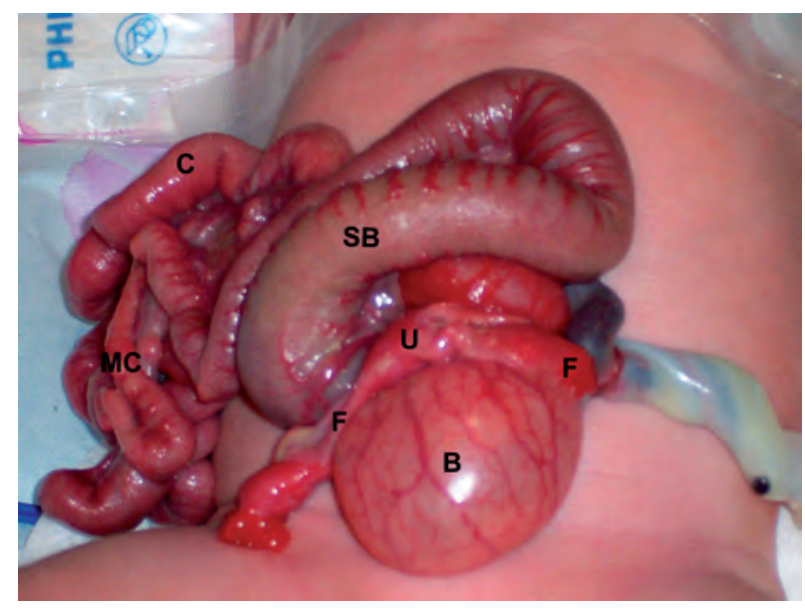

Figure 1: Gastroschisis with evisceration of stomach, colon $(\mathrm{C})$, microcolon $(\mathrm{MC})$, small bowel $(\mathrm{SB})$, bladder (B), uterus (U), Fallopian tubes (F) and ovaries.

Spot urine samples were collected daily using a bag-catch method until increased enteral feeding was tolerated successfully and the neonate was transferred from the NICU to the Medium Care Unit after the gastroschisis repair. Urinary I-FABP was measured using a commercially available I-FABP ELISA that selectively detects human I-FABP (standard: 20-5,000 pg/ml), kindly provided by HBT (Uden, the Netherlands). Measurement of urinary creatinine was performed by the department of clinical chemistry in our hospital to correct for the dilution. In a previous study, a value of $2 \mathrm{pg} / \mathrm{nmol}$ for the urinary I-FABP $(\mathrm{pg} / \mathrm{ml})$ to Creatinine $(\mathrm{Cr}, \mu \mathrm{mol} / \mathrm{ml})$ ratio allowed us to distinguish NEC or intestinal necrosis from other gastro-intestinal diagnosis. 4 Written consent was obtained from both parents prior to inclusion, and the study was conducted with approval from the local medical ethical committee.

During the first two days after the gastroschisis repair, the urinary IFABP:Cr of the patient was below the detection limit, indicating that the reduction of the organs did not lead to intestinal mucosal cell damage (Fig. 2). Two days after the operation, a small rise in urinary I-FABP:Cr from below the detection limit to $1.25 \mathrm{pg} / \mathrm{nmol}$ was found and remained stable during the next two days, after which a decline was found. Interestingly, increase of the enteral feeding, did not lead to changed urinary I-FABP:Cr levels. This observation correlates very well with the peri-operative low intravesical pressures and beneficial clinical data, such as normal temperature, good toleration of initiation and advancement of enteral feeding. 


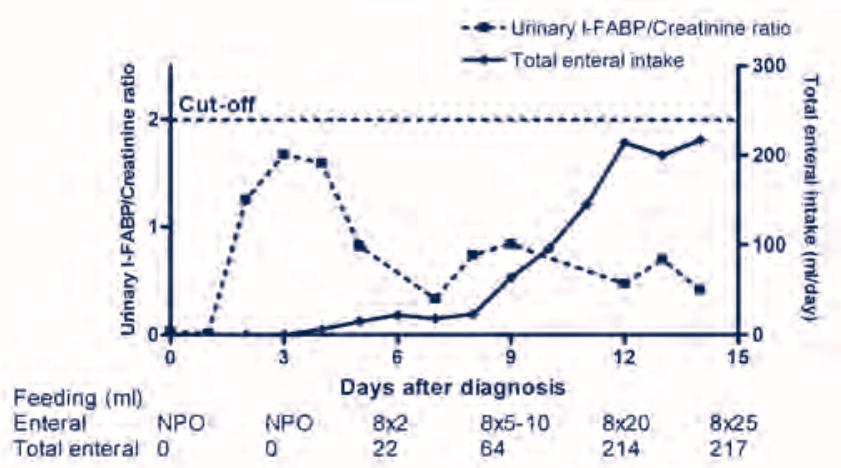

Figure 2: Reduction of herniated organs and increase of enteral feeding did not lead to significantly elevated urinary I-FABP:Creatinine levels, reflecting intestinal mucosal cell damage.

\section{Discussion}

Gastroschisis is a paraumbilical abdominal wall defect with unknown aetiology, usually right sided, through which intra-abdominal contents, commonly the intestines and stomach, eviscerate. There may be also herniation of the liver and the spleen, the Fallopian tubes, ovaries, testicles and bladder. ${ }^{1}$ The here reported herniation of the bladder is rarely observed. In an 18 year review by Novotny et al. 69 patients were studied, three (4.3\%) had bladder evisceration. $^{7}$

Fear described in 1878 a surgical repair of gastroschisis consisting of primary closure with fatal outcome. In 1966 Izant et al described manual stretching of the abdominal wall to enlarge the abdominal cavity. ${ }^{8}$ This technique is still used today, and was also performed in our case. In 1967 Schuster et al. described the first method of staged closure. ${ }^{9}$ Since Moore and Stokes described in 1953 the physiologic consequence of increased intraabdominal pressure on pulmonary function, surgeons have tried to minimize the sudden increased abdominal pressure after closure of the abdominal wall. ${ }^{10}$

So far, diagnosis of elevated intra-abdominal pressure during and after reduction of organs is based on invasive, indirect measures such as determination of bladder pressure and gastric mucosal $\mathrm{PiCO}_{2} .{ }^{2,3}$ Previous studies showed that visceral circulation is well preserved when intra-abdominal pressure does not exceed $20 \mathrm{~mm} \mathrm{Hg}$ with reduced gastro-intestinal and pulmonary related morbidity and mortality as result. 3,11 


\section{Chapter 3}

Increased intra-abdominal pressure leads to hypoperfusion of the intra-abdominal organs including the kidneys and gastrointestinal tract. ${ }^{2}$ The intestinal mucosa is particularly susceptible for hypoperfusion-induced damage and is believed to be a major cause of complications in these patients. Therefore, assessment of intestinal mucosal damage may be an early indicator of increased intraabdominal pressure with reduced splanchnic blood flow and postoperative complications. In a previous study, we showed that splanchnic hypoperfusion correlates significantly with the release of small cytosolic proteins specifically located in mature enterocytes (I-FABP) into the circulation and urine. ${ }^{12}$ In line with these data, this case shows that reduction of the eviscerated organs and primary closure did neither lead to an increase of intra-abdominal pressure, measured by bladder pressure, nor to a significant elevation of urinary I-FABP:Cr levels. Furthermore, the start and increase of enteral feeding was not accompanied by significant alteration of urinary I-FABP:Cr levels. The minimally changing urinary I-FABP:Cr ratio is a representative of the continuous release of FABP originating from mature detaching or dying enterocytes or in other words the normal physiologic turnover of enterocytes. ${ }^{13}$ Unfortunately, this case report does not provide data on intestinal epithelial cell damage after enteral feeding was tolerated successfully and the neonate was transferred from the NICU to the Medium Care Unit after the gastroschisis repair. Of course, it would have been interesting to determine the ratio of urinary I-FABP:Cr when the neonate became septic and subsequently died, especially since this would have revealed whether the origin of sepsis would have been based on intestinal ischemia. However, the aim of the study was to determine intestinal mucosal cell damage with a noninvasive test during gastroschisis-repair until full enteral feeding was reached.

This case shows that intestinal mucosal cell damage, which is a major source of complications during and after gastroschisis repair, did nor occur during primary closure of the abdominal wall after gastroschisis, neither during the start and increase of enteral feeding. Further research into the value of the prospective measurement of urinary I-FABP in neonates with gastro-intestinal compromise will determine its role as a non-invasive diagnostic tool of the intestinal mucosal condition. 


\section{RefERENCES}

1. Wilson RD, Johnson MP. Congenital abdominal wall defects: an update. Fetal Diagn Ther. 2004; 19: 385-98.

2. Engum SA, Kogon B, Jensen E, Isch J, Balanoff C, Grosfeld JL. Gastric tonometry and direct intraabdominal pressure monitoring in abdominal compartment syndrome. I Pediatr Surg. 2002; 37: 214-8.

3. Olesevich M, Alexander F, Khan M, Cotman K. Gastroschisis revisited: role of intraoperative measurement of abdominal pressure. J Pediatr Surg. 2005; 40: 789-92.

4. Derikx JP, Evennett NJ, Degraeuwe PL, et al. Urine based detection of intestinal mucosal cell damage in neonates with suspected Necrotizing Enterocolitis. Gut. 2007; 56: 1473-5.

5. Lieberman JM, Sacchettini J, Marks C, Marks WH. Human intestinal fatty acid binding protein: report of an assay with studies in normal volunteers and intestinal ischemia. Surgery. 1997; 121: 335-42.

6. van de Poll MC, Derikx JP, Buurman WA, et al. Liver manipulation causes hepatocyte injury and precedes systemic inflammation in patients undergoing liver resection. World J Surg. 2007; 31: 2033-8.

7. Novotny DA, Klein RL, Boeckman CR. Gastroschisis: an 18-year review. J Pediatr Surg. 1993; 28: 650-2.

8. Izant RJ, Jr., Brown F, Rothmann BF. Current embryology and treatment of gastroschisis and omphalocele. Arch Surg. 1966; 93: 49-53.

9. Schuster SR. A new method for the staged repair of large omphaloceles. Surg Gynecol Obstet. 1967; 125: 837-50.

10. Moore TC, Stokes GE. Gastroschisis; report of two cases treated by a modification of the gross operation for omphalocele. Surgery. 1953; 33: 112-20.

11. Lacey SR, Carris LA, Beyer AJ 3rd, Azizkhan RG. Bladder pressure monitoring significantly enhances care of infants with abdominal wall defects: a prospective clinical study. J Pediatr Surg. 1993; 28: 1370-4.

12. Derikx JP, Poeze M, van Bijnen AA, Buurman WA, Heineman E. Evidence for intestinal and liver epithelial cell injury in the early phase of sepsis. Shock. 2007; 28: 544-8.

13. Derikx JP, Blijlevens NM, Donnelly JP, et al. Loss of enterocyte mass is accompanied by diminished turnover of enterocytes after myeloablative therapy in haematopoietic stem cell transplant recipients. Ann Oncol. 2008, in press. 


\subsection{Neutrophil activation products: potential new plasma markers for diagnosis of patients with acute appendicitis}

Geertje Thuijls *, Joep P. M. Derikx *, Fred J. Prakken, Bregje Huisman, Annemarie A. van Bijnen, LWErnest van Heurn, Wim A. Buurman, Erik Heineman * Equally contributed to this work Submitted. 


\begin{abstract}

\section{Background}

Diagnosis of acute appendicitis (AA) remains a surgical dilemma, with negative appendectomy rates of $5-40 \%$, and perforation suggestive for late operative intervention in 5-30\%. The aim of this study is to evaluate new plasma markers, representing early neutrophil activation, to improve diagnostic accuracy in patients suspected for AA.
\end{abstract}

\title{
Methods
}

Sixty patients who underwent surgery for suspected AA were included $(\mathrm{M}: F=31: 29)$ and blood sampled. Plasma concentrations of three neutrophil proteins were measured: myeloperoxidase (MPO), lactoferrin (LF) and calprotectin (CP). Controls consisted of 27 healthy volunteers.

\section{Results}

Mean plasma concentrations for MPO, LF and CP were significantly higher in 51 patients with proven AA (respectively: $107 \mathrm{ng} / \mathrm{ml}, 983 \mathrm{ng} / \mathrm{ml}, 824 \mathrm{ng} / \mathrm{ml}$ ), than in 27 healthy volunteers (respectively: 48ng/ml, $\mathrm{p}<0.01 ; 210 \mathrm{ng} / \mathrm{ml}, \mathrm{p}<0.001$; $248 \mathrm{ng} / \mathrm{ml}, \mathrm{p}<0.001)$. The plasma concentration for CP was significantly higher in 51 patients with proven AA, than in 9 patients without proven AA $(824 \mathrm{ng} / \mathrm{ml}$ versus $426 \mathrm{ng} / \mathrm{ml}, \mathrm{p}<0.05)$. Area under the curve of MPO, LF and CP was 0.81 , 0.95 and 0.91. Cut-off values, evaluated with ROC curves for MPO (54ng/ml), LF $(310 \mathrm{ng} / \mathrm{ml})$ and CP $(385 \mathrm{ng} / \mathrm{ml})$, showed clinically relevant positive likelihood ratios (LRs) of 2.81, 8.60, 6.86 and negative LRs of 0.33, 0.16, 0.05, respectively.

\section{Conclusion}

The circulating neutrophil activation product CP is significantly increased in patients with proven AA compared to patients without proven AA and healthy volunteers, making $\mathrm{CP}$ a promising new marker for AA diagnosis. 


\section{Chapter 3}

\section{INTRODUCTION}

Acute appendicitis (AA) is a common clinical entity with significant morbidity and mortality, particularly at the extremes of age, mainly because of the atypical clinical presentation leading to a low diagnostic and therapeutic accuracy ${ }^{1-3}$.In general, the risk of 2 primary adverse outcomes must be balanced in diagnosis and consequent management of suspected AA: firstly, misdiagnosis leads to removal of a normal appendix and secondly, delayed diagnosis may lead to perforation and peritonitis. Since delayed diagnosis and treatment of $\mathrm{AA}$ are associated with an increased rate of perforation, with resulting increased morbidity and mortality rates, timely intervention is crucial ${ }^{1}$.To reduce the risk of appendiceal perforation, surgeons have traditionally favored early appendectomy. As a consequence, the appendix is normal in approximately 5-40 \% of patients who undergo explorative laparotomy for suspected AA ${ }^{4}$. However, perforation of an inflamed appendix still occurs in 5$30 \%$ of patients treated surgically for suspected AA ${ }^{4}$.

To improve the diagnostic accuracy of patients suspected for AA, the value of diagnostic imaging modalities and conventional inflammatory parameters has been studied. Studies performed in research environments report good results for diagnostic imaging in patients with clinically high probability of AA 5-7. However, a population-based cohort study showed that the frequency of misdiagnosis leading to unnecessary appendectomy and the frequency of perforation did not change with the introduction of computed tomography and ultrasonography ${ }^{8}$. No major improvement of diagnostic accuracy was found using white blood cell count (WBC) and C-reactive protein (CRP) ${ }^{9,10}$.

The pathophysiology of AA is characterized by defects in or increased permeability of the mucosal barrier, leading to inflammation and concomitant sequestration of large numbers of neutrophils into the gut wall ${ }^{1}$.The neutrophil proteins myeloperoxidase (MPO), lactoferrin (LF) and calprotectin (CP) are released upon neutrophil activation and readily detectable in feces, as observed in patients with inflammatory bowel disease ${ }^{11-14}$. Appendicitis is an acute inflammatory disease, which makes a fecal test too time-consuming and not suitable for diagnosis of acute disease onset.

Diagnostic tests aim at discriminating between clinically "normal" and "abnormal". Research to develop new diagnostic tests should subsequently answer the following research questions: Phase 1 question: Do patients with the target disorder have different test results from normal individuals? The answer requires comparison of test results among patients with the disease and people known not to have the disease (healthy individuals). Phase 2 question: Are 
patients with certain test results more likely to have the target disorder than patients with other test results? This question is also answered by comparison of test results among patients with the disease and healthy individuals, but now test characteristics such as sensitivity, specificity and likelihood ratio are estimated. Phase 3 question: Among patients in whom it is clinically sensible to suspect the target disorder, does the test result distinguish those with and without target disorder? To get the appropriate answer, a consecutive series of such patients should be studied. Phase 4 question: Do patients who undergo the diagnostic test fare better (in their ultimate health outcomes) than similar patients who do not undergo the diagnostic test? The question have to be answered by randomizing patients to undergo the test of interest or another (or no) test ${ }^{15}$.

This pilot study is the first to investigate the usefulness of circulating neutrophil activation products MPO, LF and CP in diagnosing AA. Therefore we address the phase 1 and 2 questions.

\section{MATERIALS AND METHODS}

\section{Patients}

From May 2004 to July 2005, 60 patients suspected for acute AA who underwent appendectomy were consecutively included at the emergency department of the University Hospital of Maastricht (31 males, 29 females; mean age 33 years, age range 6-77 years). Also 27 healthy volunteers were included in this study to obtain reference values for plasma concentrations of MPO, LF, CP and CRP (20 males, 7 females; mean age 24 years, age range 361 years). Informed consent was obtained from each participant, and the study was approved by the local ethics committee.

On admission to the emergency department EDTA plasma samples were collected and stored at $-20^{\circ} \mathrm{C}$ until analyses. Diagnosis was based on findings during surgery and histopathological examination of the appendix. Finally, 2 groups were identified. Group 1, patients with proven AA, is the group with confirmed appendicitis by histopathology, ( $n=51,28$ males, 23 females; mean age 34 years, range 6-77 years). Out of the 51 appendicitis specimens, 4 (8\%) showed acute mucosal inflammation indicating catarrhal appendicitis. Thirtyseven $(72.5 \%)$ showed signs of a suppurative (phlegmonous) appendix, and in 10 cases $(19.5 \%)$ the final diagnosis of a gangrenous (necrotizing) appendix was defined. Group 2, patients without proven AA, consists of patients with suspicion of appendicitis without AA ( $n=9,3$ males, 6 females; mean age 30 years, age range 11-52 years), based on findings during laparoscopic surgery 


\section{Chapter 3}

$(n=3)$ or histopathological examination $(n=6)$. The diagnoses in these patients without AA were gynaecological problems $(n=2)$, diverticulitis $(n=1)$, fecal impaction $(n=1)$, unknown $(n=5)$.

\section{Methods}

MPO, LF and CP concentrations in plasma samples were determined using standard ELISAs kindly provided by HyCult biotechnology ( $\mathrm{Hbt}$, Uden, the Netherlands). The detection limits for MPO, LF and CP were $4 \mathrm{ng} / \mathrm{ml}, 50 \mathrm{ng} / \mathrm{ml}$ and $10 \mathrm{ng} / \mathrm{ml}$, respectively. The human MPO, LF and CP ELISA kits are solidphase enzyme-linked immunosorbent assays based on the sandwich principle with a working time of 3 ? hours. CRP and WBC concentration were measured for routine patient care and retrospectively collected.

\section{Statistics}

Statistical analysis was performed with GraphPad Prism 4 for Windows (GraphPad Software Inc., San Diego, CA). Normality was tested using Kolmogorov-Smirnov test and normal distribution was found for plasma CP and WBC. The distribution of plasma MPO, LF and CRP did not pass the normality test. Therefore, one-way ANOVA, with post-hoc Bonferroni multiple comparison test, was used for between group comparisons for CP. Unpaired T-test was used for WBC. KruskalWallis, with post hoc Dunn's multiple comparison test, was used for between group comparisons for MPO, LF and CRP. Data are presented as mean \pm standard error (SEM).

In order to find the cutoff points of plasma MPO, LF, CP and CRP levels that most accurately discriminate patients with AA from healthy volunteers, Receiver Operating Characteristics (ROC) curves were drawn by plotting sensitivity against 1 -specificity for all MPO, LF, CP and CRP thresholds. The overall accuracy of the plasma markers was summarized using area under the curve (AUC). Best cutoff point is defined as the maximum sum of sensitivity and specificity. These cutoff points were used to calculate sensitivity, specificity, positive and negative likelihood ratios (LRs). $\mathrm{P}<0.05$ was considered statistical significant. 


\section{ResULTS}

Of the 60 patients undergoing surgery for suspected AA, 51 had histopathologically confirmed AA and 9 patients had a normal appendix confirmed during surgery $(n=3)$ or by histopathological examination $(n=6)$. Mean plasma concentrations for MPO, LF and CP were all higher in the 51 patients with proven AA (respectively: $107 \pm 12.07 \mathrm{ng} / \mathrm{ml}, 983 \pm 154.7 \mathrm{ng} / \mathrm{ml}, 824 \pm 70.03$ $\mathrm{ng} / \mathrm{ml}$ ), than in 27 healthy volunteers (respectively: $48 \pm 2.28 \mathrm{ng} / \mathrm{ml}, 210 \pm$
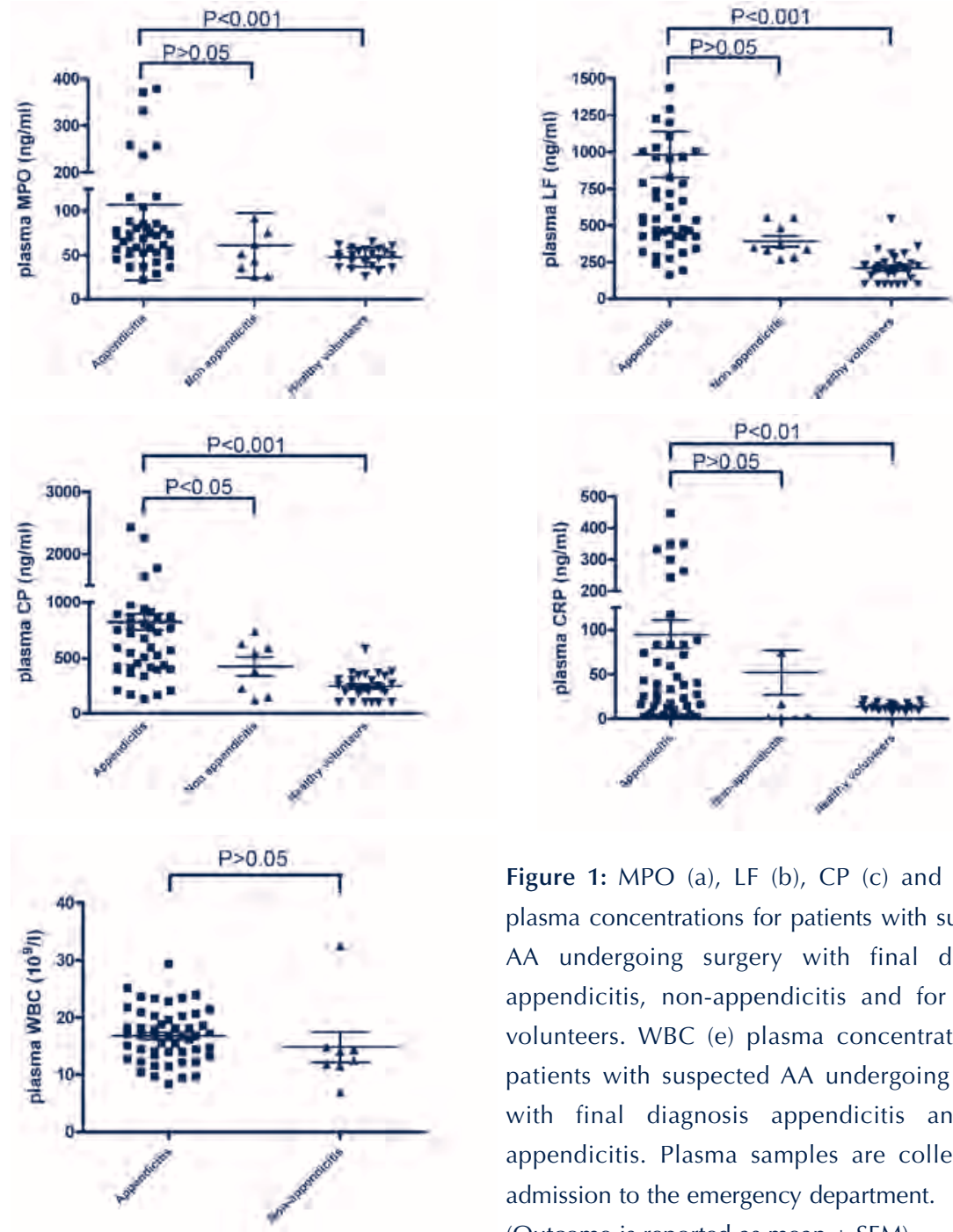

Figure 1: MPO (a), LF (b), CP (c) and CRP (d) plasma concentrations for patients with suspected AA undergoing surgery with final diagnosis appendicitis, non-appendicitis and for healthy volunteers. WBC (e) plasma concentrations for patients with suspected AA undergoing surgery with final diagnosis appendicitis and nonappendicitis. Plasma samples are collected on admission to the emergency department.

(Outcome is reported as mean \pm SEM) 


\section{Chapter 3}

$18.30 \mathrm{ng} / \mathrm{ml}, 248 \pm 22.17 \mathrm{ng} / \mathrm{ml}, \mathrm{p}<0.001$ ) (Figure $1 \mathrm{a}, \mathrm{b}$ and c). The plasma concentration for $\mathrm{CP}$ was significantly higher in 51 patients with proven $\mathrm{AA}$, than in 9 patients without proven AA $(824 \pm 70.03 \mathrm{ng} / \mathrm{ml}$ versus $426 \pm 83.86$ $\mathrm{ng} / \mathrm{ml}, \mathrm{p}<0.05)$, while there was no statistically significant difference in MPO and LF plasma concentration between patients with proven AA and patients without proven AA (respectively: $107 \pm 12.07 \mathrm{ng} / \mathrm{ml}$ versus $61 \pm 12.16 \mathrm{ng} / \mathrm{ml}$ and $983 \pm 154.7 \mathrm{ng} / \mathrm{ml}$ versus $392 \pm 36.56 \mathrm{ng} / \mathrm{ml}, \mathrm{p}>0.05)$.

The ideal cut-off values evaluated with ROC to discriminate patients with AA from healthy volunteers yielding the maximum sum of sensitivity and specificity, for MPO $(54 \mathrm{ng} / \mathrm{ml}), \mathrm{LF}(310 \mathrm{ng} / \mathrm{ml})$ and CP $(385 \mathrm{ng} / \mathrm{ml})$, showed a specificity of $73 \%, 90 \%, 86 \%$ and a sensitivity of $76 \%, 86 \%, 96 \%$, respectively (Figure 2). This resulted in positive LRs of 2.81, 8.60, 6.86 and negative LRs of $0.33,0.16$ and 0.05 , respectively. The area under the curve for MPO, LF and CP in the diagnosis of AA was 0.81 (0.71-0.91), 0.95 (0.90-1.0) and 0.91 (0.85-0.98) (Table 1).

Mean plasma concentration for CRP was higher in the 51 patients with proven AA $(94.9 \pm 15.54 \mathrm{ng} / \mathrm{ml})$, than in 27 healthy volunteers $(13.4 \pm 0.90 \mathrm{ng} / \mathrm{ml}$, $\mathrm{P}<0.01$ ) (Figure $1 \mathrm{~d}$ ). The ideal cut-off value evaluated with ROC to discriminate patients with AA from healthy volunteers for CRP showed a specificity of $75 \%$ and a sensitivity of $76 \%$. The resulting positive LR was 3.04 and the negative LR was 0.32. The area under the curve was $0.77(0.66-0.88)$ (Table 1).

Table 1: Overall accuracy (AUC) and cutoff points, defined as the maximum sum of sensitivity and specificity, using ROC curves, with corresponding sensitivity, specificity, positive and negative LRs for MPO, LF and CP to differentiate between patients with appendicitis and healthy volunteers.

\begin{tabular}{lcccccc} 
Marker & $\begin{array}{c}\text { Conc. } \\
(\mathrm{ng} / \mathrm{ml})\end{array}$ & Sensitivity & Specificity & LR+ & LR- & AUC (95\% Cl) \\
\hline MPO & 54.1 & 0.76 & 0.73 & 2.81 & 0.33 & $0.81(0.71-0.91)$ \\
LF & 309.6 & 0.86 & 0.90 & 8.60 & 0.16 & $0.95(0.90-1.0)$ \\
CP & 384.6 & 0.96 & 0.86 & 6.86 & 0.05 & $0.91(0.85-0.98)$ \\
CRP & 15.9 & 0.76 & 0.75 & 3.04 & 0.32 & $0.77(0.66-0.88)$ \\
\hline
\end{tabular}

$\mathrm{MPO}=$ myeloperoxidase, $\mathrm{LF}=$ lactoferrin, $\mathrm{CP}=$ calprotectin, $\mathrm{CRP}=\mathrm{C}$-reactive protein, $\mathrm{LR}=$ likelihood ratio.

Both CRP and WBC plasma concentrations were not statistically significant different between patients with proven $\mathrm{AA}$ and patients without proven AA (respectively: $94.9 \pm 15.54 \mathrm{ng} / \mathrm{ml}$ versus $52.1 \pm 25.04 \mathrm{ng} / \mathrm{ml}$ and $16.8^{*} 109 \pm$ $0.64 * 109 / \mathrm{L}$ versus $14.8^{*} 109 \pm 2.69 * 109 / \mathrm{L}, \mathrm{p}>0.05$ ) (Figure $1 \mathrm{~d}$ and e). 


\section{Discussion}

Early and correct diagnosis for appendicitis is crucial at the one hand to prevent perforation with resultant morbidity, and at the other hand to avoid unnecessary surgery. Surgeons rely on disease history and clinical examination for diagnosing AA. Laboratory investigations (CRP, WBC) and expensive advanced imaging techniques can help to improve diagnostic accuracy. As promising as these tests might seem, diagnostic accuracy improved only marginally in recent decades and as a consequence both negative appendectomy rates and perforation rates remain high ${ }^{4,8}$. In line with recent literature the sensitivity, specificity and LRs for CRP reported in this study are not sufficient for diagnosing appendicitis with a very high probability.

In this pilot study, addressing the phase 1 and 2 question, to analyze new tests for the diagnosis of AA we evaluated the usefulness of the neutrophil activation markers MPO, LF and CP to improve diagnostic accuracy in patients with suspected AA. The tests are relatively simple and cheap ELISA assays that can be performed in clinical laboratories within 4 hours. The proteins showed clinically relevant positive LRs, especially for LF and CP, and negative LRs. LRs are used and considered as most important for evaluation of a clinical test, because they summarize information on both sensitivity and specificity and provide discriminative power of the test. LRs are clinical relevant and applicable because they change the diagnostic probability to a working diagnosis, showing how a particular test result predicts the risk of disease. Furthermore LRs are independent of the prevalence of disease among the studied patients, unlike positive and negative predictive values, and can therefore be generalized to other settings ${ }^{16,17}$.

Elevated WBC levels measured in patients with appendicitis are mainly caused by increased neutrophil numbers. During appendicitis the transformation of neutrophils from passively circulating cells to potent effector cells is mediated by exocytosis of granules and cytosolic proteins leading to the release of MPO, LF and CP. Therefore, we consider neutrophil activation products more specific for detection of appendicitis then WBC. Neutrophil granules contain antimicrobial and potentially cytotoxic substances that are delivered to the exterior of the cell following degranulation. MPO and LF are present in polymorphonuclear neutrophil granules. MPO $(150 \mathrm{kDa})$ is a microbicidal hemoprotein stored in azurophilic granules of neutrophils and macrofages. MPO produces hypochlorous acid and tyrosyl radicals, both cytotoxic agents that kill bacteria ${ }^{18}$. LF (78 kDa) is a glycoprotein mainly stored in specific granules. Upon activation it is called lactoferricin and possesses antimicrobial 


\section{Chapter 3}

activity against a broad spectrum of Gram-positive and Gram-negative bacteria. The antimicrobial activity is exerted by impairment of bacterial growth by sequestration of iron, and by causing bacterial cell lysis by binding to the cell membrane ${ }^{18}$. CP $(36 \mathrm{kDa})$ is a heterodimer of two calcium binding cytosolic proteins, MRP14 and MRP8, constituting $60 \%$ of soluble proteins in neutrophils. CP is released upon neutrophil activation, and has bacteriostatic and cytokine-like effects in the local environment ${ }^{12}$. CP is also present in monocytes and mucosal epithelial cells ${ }^{19}$. Therefore leakage of epithelial cells can also contribute to elevated plasma levels of CP. Recently Sudan et al. showed that fecal CP is a useful marker for intestinal allograft monitoring ${ }^{19}$. Fecal CP levels were significantly higher in patients with allograft rejection than in patients with viral or non-specific enteritis. Research on inflammatory bowel disease showed elevation of fecal CP in patients with active disease, with good correlation between amount of $\mathrm{CP}$ and the degree of mucosal damage measured endoscopically and between amount of $\mathrm{CP}$ and the extensiveness of inflammation assessed by leukocyte scan ${ }^{13,20,21}$.

$\mathrm{CP}$ is the most promising marker for diagnoses of acute appendicitis, with high positive LR and a low negative LR. Relevant characteristics of CP in this respect are the fact that it is released immediately upon inflammation and epithelial cell damage, both present in appendicitis. In this study the phase 1 and 2 questions comparing test results between patients with appendicitis to healthy controls are answered. Furthermore CP plasma levels are significantly higher in patients operated for suspected AA with final diagnosis AA compared to patients without AA. In conclusion; a relatively simple and low cost ELISA assay seems of value for diagnosing AA.

Next step is to study the phase 3 question; the accuracy of these markers in all patients admitted to the hospital with a clinical suspicion of AA. Furthermore, we suggest that a single marker may not be sufficient for diagnosing appendicitis with a very high probability. Further tests should be developed to combine these with the promising marker CP and thereby achieve even higher likelihood ratios. 


\section{RefERENCES}

1. Prystowsky JB, Pugh CM, Nagle AP. Current problems in surgery. Appendicitis. Curr Probl Surg 2005; 42:688-742.

2. Humes DJ, Simpson J. Acute appendicitis. Bmj 2006; 333:530-4.

3. Bundy DG, Byerley JS, Liles EA, et al. Does this child have appendicitis? Jama 2007; 298:438-51.

4. Andersson RE. Meta-analysis of the clinical and laboratory diagnosis of appendicitis. $\mathrm{Br}$ J Surg 2004; 91:28-37.

5. Kaiser S, Frenckner B, Jorulf HK. Suspected appendicitis in children: US and CT--a prospective randomized study. Radiology 2002; 223:633-8.

6. Gwynn LK. The diagnosis of acute appendicitis: clinical assessment versus computed tomography evaluation. J Emerg Med 2001; 21:119-23.

7. Rao PM, Rhea JT, Novelline RA, et al. Effect of computed tomography of the appendix on treatment of patients and use of hospital resources. N Engl / Med 1998; 338:141-6.

8. Lee SL, Walsh AJ, Ho HS. Computed tomography and ultrasonography do not improve and may delay the diagnosis and treatment of acute appendicitis. Arch Surg 2001; 136:556-62.

9. Beltran MA, Almonacid J, Vicencio A, et al. Predictive value of white blood cell count and C-reactive protein in children with appendicitis. J Pediatr Surg 2007; 42:1208-14.

10. Eriksson S, Granstrom L, Olander B, et al. Sensitivity of interleukin-6 and C-reactive protein concentrations in the diagnosis of acute appendicitis. Eur J Surg 1995; 161:415.

11. Carroll D, Corfield A, Spicer R, et al. Faecal calprotectin concentrations and diagnosis of necrotising enterocolitis. Lancet 2003; 361:310-1.

12. Fagerhol MK. Calprotectin, a faecal marker of organic gastrointestinal abnormality. Lancet 2000; 356:1783-4.

13. Langhorst J, Elsenbruch S, Koelzer J, et al. Noninvasive Markers in the Assessment of Intestinal Inflammation in Inflammatory Bowel Diseases: Performance of Fecal Lactoferrin, Calprotectin, and PMN-Elastase, CRP, and Clinical Indices. Am J Gastroenterol 2008; 103:162-9.

14. Sugi K, Saitoh O, Hirata I, et al. Fecal lactoferrin as a marker for disease activity in inflammatory bowel disease: comparison with other neutrophil-derived proteins. Am J Gastroenterol 1996; 91:927-34.

15. Sackett DL, Haynes RB. The evidence base of clinical diagnosis. London: BMJ Publishing Group; 2002.

16. Deeks JJ, Altman DG. Diagnostic tests 4: likelihood ratios. Bmj 2004; 329:168-9.

17. Akobeng AK. Understanding diagnostic tests 2: likelihood ratios, pre- and post-test probabilities and their use in clinical practice. Acta Paediatr 2007; 96:487-91. 


\section{Chapter 3}

18. Faurschou M, Borregaard N. Neutrophil granules and secretory vesicles in inflammation. Microbes Infect 2003; 5:1317-27.

19. Sudan D, Vargas L, Sun Y, et al. Calprotectin: a novel noninvasive marker for intestinal allograft monitoring. Ann Surg 2007; 246:311-5.

20. Schoepfer AM, Trummler M, Seeholzer P, et al. Discriminating IBD from IBS: Comparison of the test performance of fecal markers, blood leukocytes, CRP, and IBD antibodies. Inflamm Bowel Dis 2007; 14:32-9.

21. Tibble J, Teahon $K$, Thjodleifsson B, et al. A simple method for assessing intestinal inflammation in Crohn's disease. Gut 2000; 47:506-13. 


\subsection{Urine based detection of intestinal mucosal cell damage in neonates with suspected necrotising enterocolitis}

Joep P. M. Derikx, Nicholas J. Evennett, Pieter L. J. Degraeuwe, Twan L. Mulder, Annemarie A. van Bijnen, L. W. Ernest van Heurn, Wim A. Buurman, Erik Heineman

Gut (2007); 56: 1473-5. 


\section{Chapter 3}

Necrotizing Enterocolitis (NEC) is a severe gastrointestinal disease with a mortality of $20-40 \%$, affecting predominantly premature neonates. ${ }^{1}$ In particular in the early phase of the disease, NEC continues to present a considerable diagnostic challenge to the attending clinician. The signs and symptoms of NEC are frequently non-specific, including gastro-intestinal problems such as abdominal distension and feeding intolerance, which are also amongst the most prevalent presenting features in neonatal sepsis. ${ }^{2}$ The diagnosis is further hampered by the limited diagnostic accuracy of the laboratory and radiological tests currently in use. 3,4

Histopathologically, NEC is characterized by intestinal coagulative or ischemic necrosis, starting at the mucosa and extending into the submucosa and muscularis externa. ${ }^{1}$ We sought a non-invasive test to find evidence of enterocyte cell death in infants with gastrointestinal symptoms suspicious for NEC, in order to differentiate NEC from other neonatal diseases that present with abdominal signs.

Intestinal Fatty Acid Binding Protein (I-FABP) has been reported to be a useful plasma marker for early enterocyte cell death. ${ }^{5,6}$ The small (14-15 kDa) cytosolic I-FABP is specifically present in mature enterocytes of small and large intestine and released as soon as the cell membrane integrity is compromised. I-FABP is present in very low amounts in plasma of healthy individuals, likely representing the normal turnover of enterocytes, but levels rapidly rise after episodes of acute intestinal ischemia and inflammation, including NEC. ${ }^{5-8}$

Due to its small molecular weight, I-FABP present in the systemic circulation passes through the glomerular filter (fractional renal excretion: $28 \%$, half-life time: 11 minutes) and can readily be detected in urine. ${ }^{9}$ I-FABP is not expressed in the urinary tract mucosa. Therefore, urinary values of I-FABP provide specific information about the number of dying intestinal epithelial cells. Given the age and birth weight of our population of interest, there are important advantages to sampling urine instead of plasma. ${ }^{10,11}$

Over an 18 month period, 17 consecutive neonates were identified in the neonatal intensive care unit at the University Hospital Maastricht, in whom NEC was suspected. Inclusion criteria were the presence of at least one clinical gastrointestinal sign (abdominal distension or discoloration, increased gastric residues, bloody stools), causing sufficient clinical concern to require an abdominal X-ray (AXR), and/or to stop enteral feeding. Written consent was obtained from both parents prior to inclusion, and the study was conducted with approval from the local medical ethical committee.

Urine was collected daily using a bag-catch method for at least one week once appropriate consent was obtained. All neonates produced urine at a 
minimal rate of $1.9 \mathrm{ml} / \mathrm{kg} / \mathrm{hr}$. Samples were centrifuged at 4,000 x G for 15 minutes, aliquoted and stored at $-20^{\circ} \mathrm{C}$ until analysis. Urinary I-FABP was measured using a commercially available I-FABP ELISA that selectively detects human I-FABP (standard: $20-5,000 \mathrm{pg} / \mathrm{ml}$ ), kindly provided by HBT (Uden, the Netherlands). Values were expressed as a ratio (units $\mathrm{pg} / \mathrm{nmol}$ ) of I-FABP $(\mathrm{pg} / \mathrm{ml}$ ) to creatinine $(\mathrm{Cr}, \mu \mathrm{mol} / \mathrm{l})$, in order to compensate for variations in the concentration of the urine. Urinary I-FABP:Cr levels were compared between neonates with NEC and other final diagnoses with Mann Whitney test.

Five out of the 17 infants with suspected NEC subsequently developed NEC stage Ila (pneumatosis intestinalis on AXR, confirmed by a paediatric radiologist) or higher. Of the remaining 12 patients suspected for NEC, there were seven cases of sepsis, one ileal atresia with intestinal necrosis, two patients for whom a diagnosis of constipation was ultimately given, one patient with Hirschsprung's disease, and finally one patient born with gastroschisis who later developed signs and symptoms of NEC after gastroschisis-repair. In the neonates who developed NEC, the average gestational age was 32 3/7 weeks, the average age at diagnosis was 14 days, and the average birth weight was 1,500g. For the neonates who subsequently received different diagnoses, the average gestational age and weight were $304 / 7$ weeks and $1,487 \mathrm{~g}$, respectively.

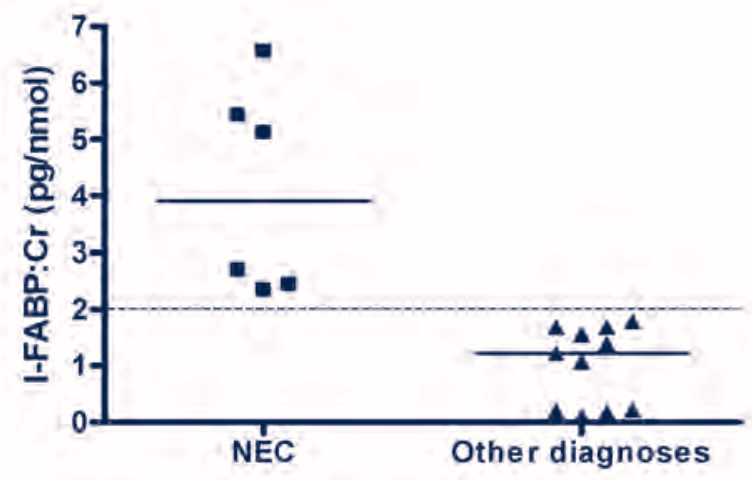

Figure 1: In the first urine sample of neonates with suspected necrotising enterocolitis (NEC), the mean urinary I-FABP:Cr ratio was significantly higher in neonates who ultimately developed NEC or intestinal necrosis $(3.9 \mathrm{pg} / \mathrm{nmol}$, range 2.3 to 6.6$)$ than in those without NEC $(1.2 \mathrm{pg} / \mathrm{nmol}$, range 0.1 to 1.8$)(p=0.001)$. Cr, creatine; I-FABP, intestinal fatty acid binding protein. 


\section{Chapter 3}

In the first urine sample, the median urinary I-FABP:Cr ratio was significantly higher in neonates who ultimately developed NEC or intestinal necrosis (3.9, range: 2.3-6.6 $\mathrm{pg} / \mathrm{nmol})$ than in patients without NEC (1.2, range: 0.1-1.8 $\mathrm{pg} / \mathrm{nmol}, \mathrm{p}=0.001$ ) (Fig 1). A value of $2 \mathrm{pg} / \mathrm{nmol}$ could distinguish neonates with NEC or intestinal necrosis from the other neonates. None of the patients without either NEC or intestinal necrosis were found to have an I-FABP:Cr ratio in excess of $2 \mathrm{pg} / \mathrm{nmol}$ over the first week.

In the present study urinary I-FABP, a specific marker for intestinal mucosal damage, was measured using ELISA to identify patients with NEC. This study is the first to show that measurement of urinary I-FABP offers valuable additional information in the early diagnosis of NEC. Furthermore, whilst previous studies have assessed the feasibility of plasma I-(and L-)FABPs in identifying patients with NEC when compared to healthy controls 7,8 , this is the first study which prospectively identifies neonates with NEC, amongst a population of preterm infants who present with gastrointestinal symptoms.

In our small series, a value of $2 \mathrm{pg} / \mathrm{nmol}$ for the urinary I-FABP:Cr ratio was accurate to distinguish the group with NEC or intestinal necrosis from the other diagnoses.

NEC is a complex and likely multi-factorial disease for which the aetiology remains incompletely understood. However, intestinal epithelial cell death is an important early hallmark ${ }^{1}$ which is in line with the observed increased urinary I-FABP. Moreover, normalization of the urinary I-FABP:Cr ratio correlated well with clinical improvement, at least in the short term.

Given the ease and frequency with which urine can be collected, without adverse consequences for the baby, the feasibility of urinary I-FABPs as a screening tool for NEC needs to be further evaluated in a prospective trial. Finally, as the cumulative release of I-FABP can be used to estimate the extent of intestinal damage, it may also help to guide treatment strategies, such as the timing of surgery (if required), the duration of antibiotic therapy, and selecting the ideal time at which enteral feeding should be re-initiated. 


\section{RefERENCES}

1. Lin PW, Stoll BJ. Necrotising enterocolitis. Lancet 2006;368:1271-83.

2. Fanaroff $\mathrm{AA}$, Korones $\mathrm{SB}$, Wright $\mathrm{LL}$, et al. Incidence, presenting features, risk factors and significance of late onset septicemia in very low birth weight infants. Paediatr Infect Dis / 1998;17:593-8.

3. Hällströma $M$, Koivistob A, Janasa $M$, et al. Laboratory parameters predictive of developing necrotizing enterocolitis in infants born before 33 weeks of gestation. J Pediatr Surg 2006;41:792- 8.

4. Tam Alda L, Camberos A, Applebaum H. Surgical Decision Making in Necrotizing Enterocolitis and Focal Intestinal Perforation: Predictive Value of Radiologic Findings. J Pediatr Surg 2002;37:1688-91.

5. Kanda T, Fujii H, Tani T, et al. Intestinal fatty acid-binding protein is a useful diagnostic marker for mesenteric infarction in humans. Gastroenterology 1996;110:339-43.

6. Lieberman JM, Sacchettini J, Marks C, et al. Human intestinal fatty acid binding protein: Report of an assay with studies in normal volunteers and intestinal ischemia. Surgery 1997;121:335-42.

7. Edelson MB, Sonnino RE, Bagwell CE, et al. Plasma Intestinal Fatty Acid Binding Protein in Neonates With Necrotizing Entercolitis: A Pilot Study. J Pediatr Surg 1999;34:1452-7.

8. Guthmann F, Börchers T, Wolfrum C, et al. Plasma concentration of intestinal- and liver$\mathrm{FABP}$ in neonates suffering from necrotizing enterocolitis and in healthy preterm neonates. Mol Cell Biochem 2002;239:227-34.

9. Poll MCG van de, Derikx JPM, Buurman WA, et al. Liver manipulation causes hepatocyte injury and precedes systemic inflammation in patients undergoing liver resection. World J Surg, conditionally accepted.

10. Smoller BR, Kruskall MS. Phlebotomy for diagnostic laboratory tests in adults. Pattern of use and effect on transfusion requirements. N Engl J Med 1986;314:1233-35.

11. Lin JC, Strauss RG, Kulhavy JC, et al. Phlebotomy overdraw in the neonatal intensive care nursery. Pediatrics 2000;106:e19-25. 


\subsection{Diagnostic accuracy of new non-invasive markers for diagnosing Necrotizing Enterocolitis: urinary I-FABP, urinary claudin-3 and faecal calprotectin}

Geertje Thuijls, Joep P. M. Derikx, Pieter L. J. Degraeuwe, Twan L. Mulder, Annemarie A. van Bijnen, L. W. Ernest van Heurn, Wim A. Buurman, Erik Heineman Submitted. 


\begin{abstract}

\section{Background}

Diagnosis of the severe gastrointestinal neonatal disease necrotising enterocolitis (NEC) remains challenging since clinical, radiological and laboratory markers lack specificity. The aim of this study is to improve diagnostic accuracy for diagnosing NEC by using non-invasive markers representing important histopathological factors; gut wall integrity loss (urinary I-FABP and urinary claudin-3) and gut wall inflammation (faecal calprotectin).

Methods. Twenty-nine consecutive neonates suspected for NEC were included and urine and faeces samples were collected. Urinary concentrations of I-FABP and claudin-3 and faecal concentration of calprotectin were measured.
\end{abstract}

\title{
Results
}

Twelve of the 29 neonates suspected for NEC developed NEC Bell stage IIA or higher. The other patients had sepsis (13), obstipation (1), morbus Hirschsprung (1), gastroschisis (1) and colitis (1). Mean I-FABP, claudin-3 and calprotectin levels were significantly higher in neonates with NEC $(5.75 \pm 0.84 \mathrm{pg} / \mathrm{nmol}$; $1267.0 \pm 175.6$ INT; $519.3 \pm 106.7 \mathrm{ug} / \mathrm{g}$ faeces) than in neonates with other final diagnosis $(0.85 \pm 0.64 \mathrm{pg} / \mathrm{nmol}, \mathrm{p}<0.0001 ; 459.2 \pm 108.1 \mathrm{INT}, \mathrm{p}=0.0003$; $103.9 \pm 39.29 \mathrm{ug} / \mathrm{g}$ faeces). Area under the receiver operating characteristic curve of I-FABP, claudin-3 and calprotectin was $1.0(p<0.001), 0.84(p=0.002)$ and $0.94(p=0.001)$.

\section{Conclusion}

The urinary markers I-FABP and claudin-3, representing gut wall integrity loss, and the faecal marker calprotectin, representing gut wall inflammation, were significantly increased in neonates with NEC compared to neonates with other final diagnosis, making them promising new markers for early diagnosis for NEC. 


\section{Chapter 3}

\section{INTRODUCTION}

Necrotizing enterocolitis (NEC) is a severe gastrointestinal disease with a high morbidity and mortality (20-40\%), affecting predominantly premature neonates ${ }^{1}$. NEC continues to present a diagnostic challenge to clinicians, in particular in the early phase of the disease. The initial clinical manifestations of NEC, including gastro-intestinal problems such as abdominal distension and feeding intolerance, are frequently non-specific and indistinguishable from other gastrointestinal disorders and sepsis ${ }^{2}$. The diagnosis is further hampered by the limited diagnostic accuracy of the laboratory and radiological tests currently in use $\mathrm{e}^{3,4}$.

NEC is pathofysiologically characterized by defects in or increased permeability of the intestinal epithelial barrier, leading to inflammation ${ }^{1,5}$. We sought non-invasive tests to find evidence of loss of the intestinal epithelial lining and gut wall inflammation in neonates with gastrointestinal symptoms suspicious for NEC, in order to differentiate NEC from other neonatal diseases that present with abdominal signs. Therefore, we used urinary and faecal markers to study the condition of enterocytes and tight-junctions, the two components comprising the intestinal epithelial lining, and intestinal wall inflammation in neonates suspected for NEC.

Intestinal Fatty Acid Binding Protein (I-FABP) has been reported to be a useful plasma marker for enterocyte cell damage ${ }^{6,7}$. I-FABP, a small (14-15 $\mathrm{kDa}$ ) cytosolic, water-soluble protein, is specifically present in mature enterocytes of small and large intestine and is released as soon as the cell membrane integrity is compromised. I-FABP is present in low amounts in plasma of healthy individuals, likely representing the normal turnover of enterocytes, but levels rapidly rise after episodes of acute intestinal ischemia and inflammation, including NEC ${ }^{6-9}$. Due to its small molecular weight, I-FABP present in the systemic circulation passes through the glomerular filter (fractional renal excretion: $28 \%$, half-life time: 11 minutes) and can readily be detected in urine ${ }^{10}$. I-FABP is not expressed in the urinary tract mucosa. Therefore, urinary values of I-FABP provide specific information about the number of dying intestinal epithelial cells.

The intestinal epithelial lining is also formed by tight junctions between neighbouring enterocytes. These tight junctions consist of a large complex of intra- and extracellular proteins, including claudins $(22 \mathrm{kDa}){ }^{11,12}$. Especially claudin-3 is an important sealing claudin, expressed in high quantity in the intestine ${ }^{11}$. Claudin-3 is found to disappear rapidly from the tight junction following hemorrhagic shock and intestinal inflammatory disease and is released into the urine 13 (own unpublished data). 
In the early phase of NEC, the disease is characterized by both loss of intestinal epithelial lining and intestinal inflammation with sequestration of large numbers of neutrophils into the gut wall ${ }^{1,5}$. Intestinal neutrofielinflux and -activation causes degranulation of calprotectin, a heterodimeric peptide (36 kDa) constituting $60 \%$ of cytosolic content of neutrophils. After neutrophil degranulation, calprotectin is readily detectable in faeces and plasma. Furthermore it is remarkably resistant to degradation by faecal bacteria, making faecal calprotectin a suitable marker for gut wall inflammation ${ }^{14-16}$.

This study aims at improving diagnostic accuracy for diagnosing NEC, by using non-invasive tests to find evidence of gut wall integrity loss and gut wall inflammation in infants with gastrointestinal symptoms suspicious for NEC. Given the age and birth weight of our population of interest, there are important advantages to sampling urine and faeces instead of plasma ${ }^{17,18}$.

\section{Patients and Methods}

\section{Patients and sample collection}

Between July 2005 and March 2008, 29 consecutive neonates were identified in the neonatal intensive care unit at the Maastricht University Medical Centre, in whom NEC was suspected. Suspicion of NEC was defined as the presence of abdominal distension, causing sufficient clinical concern to require an abdominal X-ray (AXR), and/or to stop enteral feeding. Written consent was obtained from both parents, and the study was conducted with approval from the local medical ethical committee.

Urine and faeces was collected immediately upon suspicion of NEC. Urine was obtained by placing a dental rolls cotton (Schein demedis dental, Almere, the Netherlands) in the diaper of the neonate. Once the roll was filled with urine, it was placed in a sterile $5 \mathrm{ml}$ syringe (Becton Dickinson, Oxford, UK), expressed into sterile Micronics (Micronic B.V., Lelystad, the Netherlands) and stored at $20^{\circ} \mathrm{C}$ until analysis. All neonates produced urine at a minimal rate of $1.9 \mathrm{ml} / \mathrm{kg} / \mathrm{hr}$. Faeces was sampled from the diaper and frozen at $-20^{\circ} \mathrm{C}$ until analysis. Twentyone neonates produced stool within 12 hours after they were suspected for NEC. Samples were taken an average 1.5 days before final diagnosis.

\section{Markers for systemic inflammation}

In order to study the usefulness of the classical systemic inflammatory markers in the early diagnosis of NEC, platelet count, leukocyte count, and C-reactive protein (CRP) were routinely determined in the first plasma upon suspicion of NEC by the clinical chemistry laboratory of the Maastricht University Medical Centre+. 


\section{Chapter 3}

\section{Urinary I-FABP measurement}

Urinary I-FABP was measured using a commercially available enzyme-linked immunosorbent assays (ELISA) that selectively detects human I-FABP (standard: 20-5,000 pg/ml), kindly provided by Hycult Biotechnology (Uden, the Netherlands). Values were expressed as a ratio (units $\mathrm{pg} / \mathrm{nmol}$ ) of I-FABP $(\mathrm{pg} / \mathrm{ml})$ to creatinine $(\mathrm{Cr}, \mu \mathrm{mol} / \mathrm{l})$, in order to compensate for variations in the concentration of the urine.

\section{Urinary claudin-3 measurement}

Urinary claudin-3 levels were analyzed by western blotting. Equal amounts of each sample (adjusted to urinary creatinine levels) were separated by SDSPAGE gel, transferred to PVDF-membrane and probed using primary antibody to claudin-3 (Rabbit anti-Claudin-3 (34-1700), Zymed Laboratories, San Francisco, CA). After incubation with goat anti rabbit HRP-conjugated secondary antibody (Jackson, West Grove, PA), signal was detected by supersignal west pico chemiluminescence substrate (Pierce, Etten-Leur, the Netherlands). Band intensity was semi-quantitatively analyzed using Quantity One (Biorad, Hercules, CA).

\section{Faecal calprotectin measurement}

After thawing of the faeces, $100 \mathrm{mg}$ was weighed and $4.9 \mathrm{ml}$ extraction buffer (0.1M Tris, $0.15 \mathrm{mM} \mathrm{NaCl}, 1.0 \mathrm{M}$ urea, $10 \mathrm{mM} \mathrm{CaCl} 2 \cdot 2 \mathrm{H}_{2} \mathrm{O}, 0.1 \mathrm{M}$ citric acid, $0.5 \%$ BSA, pH 8.0) was added. After 30 minutes of shaking, $1 \mathrm{ml}$ of the suspension was centrifuged at $10,000 \mathrm{rpm}$ for 20 minutes at $4^{\circ} \mathrm{C}$ and the supernatant was aliquoted and stored at $-20^{\circ} \mathrm{C}{ }^{19}$. The calprotectin concentration was measured in the lysate using the commercially available calprotectin ELISA (standard 0.78-50 ng/mL), kindly provided by Hycult Biotechnology. The faecal concentration of calprotectin is given in $\mu \mathrm{g}$ calprotectin per gram faeces.

\section{Statistical analyses}

Statistical analyses were performed with Prism 4.0 for Windows (GraphPad Software Inc. San Diego, CA). Concentrations of the studied urinary and faecal were presented as mean \pm standard error (SEM). Normality of all data obtained was verified by Kolmogorov-Smirnov test. Levels of the markers were compared between neonates who ultimately developed NEC and neonates with other final diagnoses using unpaired t-test.

Receiver operating characteristic (ROC) curves were used to study diagnostic accuracy of the markers in detecting NEC, represented by area under 


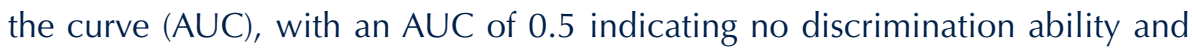
an AUC of 1.0 indicating maximal discrimination ability. A p-value below 0.05 was considered statistically significant.

The STARD statement for reporting studies of diagnostic accuracy were used in this study ${ }^{20}$ (figure 1 ).

\section{Results}

\section{Patient characteristics}

Between July 2005 and March 2008, 29 consecutive neonates were identified in the neonatal intensive care unit at the Maastricht University Medical Centre, in whom NEC was suspected. Twelve of the 29 neonates ultimately developed NEC Bell stage II (pneumatosis intestinalis on abdominal X-ray) or higher. Of the remaining 17 patients suspected for NEC 13 patients had sepsis; one had obstipation; one had morbus Hirschsprung; one had gastroschisis; one had a colitis (table 1).

No significant differences were observed between the NEC group versus the patient group with other final diagnoses in gestational age and birth weight (table 1).

Table 1: Characteristics of neonates suspected for NEC who ultimately developed NEC and other final diagnoses. No statistically significant differences were observed between the two groups.

\begin{tabular}{lll} 
& NEC & Other diagnoses \\
\hline Gestational age & $31+1$ weeks & $31+6$ weeks \\
Birth weight & 1410 grams & 1528 grams \\
Sex & $M: V=8: 4$ & $M: V=8: 9$ \\
Final diagnoses & NEC $\geq$ Bell stage II (12) & sepsis (13) \\
& & obstipation (1) \\
& & morbus Hirschsprung (1) \\
& & gastroschisis (1) \\
\hline Total & colitis (1) \\
\hline
\end{tabular}




\section{Chapter 3}

\section{Laboratory tests currently in use}

Laboratory tests currently in use for diagnosing NEC in infants with gastrointestinal symptoms suspicious for NEC are platelet count, leukocyte count, and CRP. Platelet count and leukocyte count were not statistically significant higher in the plasma samples of patients with NEC $\left(254.3^{*} 10^{3} / \mathrm{I} \pm\right.$ 96.5 and $12.2 * 10^{3} / \mathrm{I} \pm 2.5$, respectively) than in the plasma samples of neonates with other final diagnoses $\left(190.9 * 10^{3} / / \pm 30.83\right.$ and $13.2 * 10^{3} / / \pm 2.3$, respectively, $\mathrm{P}>0.05$ ) (figure $2 \mathrm{~A}+\mathrm{B}$ ). Mean plasma CRP level was statistically significant higher in the NEC group $(125.8 \mathrm{mg} / \mathrm{l} \pm 31.7)$ versus the patient group with other final diagnoses $(37.7 \mathrm{mg} / \mathrm{l} \pm 11.9, \mathrm{p}=0.0057)$ (figure 2C).

The area under the curve for CRP in the diagnosis of NEC was 0.82 $(95 \% \mathrm{Cl}=0.65-0.98, \mathrm{p}=0.007)$.
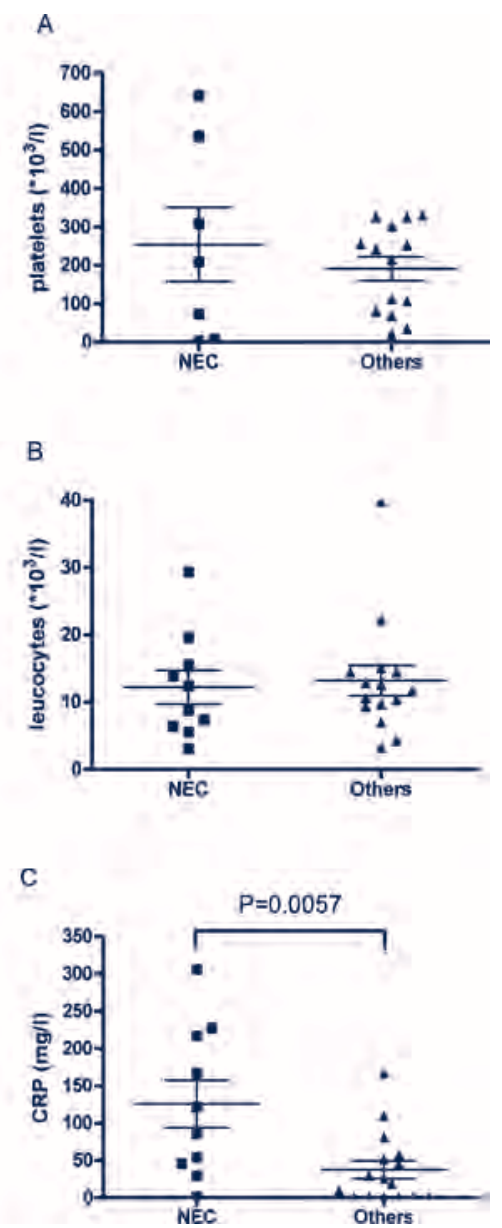

Figure 2: Laboratory tests currently in use: platelet count (2A), leucocyte count (2B), CRP (2C). Plasma platelet count and leucocyte count were not different between neonates with NEC and neonates with other final diagnoses. Mean plasma CRP level was significantly higher in neonates with NEC. 


\section{Urinary I-FABP levels was significantly higher in patients with NEC}

Mean urinary I-FABP:Cr ratio was statistically significant higher in neonates who eventually developed NEC $(5.75 \pm 0.84)$, than in those with other final diagnoses $(0.85 \pm 0.64, p<0.0001)$ (figure $3 \mathrm{~A})$. Furthermore all neonates with NEC had a urinary I-FABP:Cr value higher than $2 \mathrm{pg} / \mathrm{nmol}$, whereas all neonates without NEC had values below $2 \mathrm{pg} / \mathrm{nmol}$. A value of $2 \mathrm{pg} / \mathrm{nmol}$ discriminated between neonates with NEC and the remainder.

The area under the curve for I-FABP in the diagnosis of NEC was 1.00 $(95 \% \mathrm{Cl}=1.00-1.00, \mathrm{p}<0.001)$.

\section{Urinary Claudin-3 levels was significantly higher in patients with NEC}

Recently it has been discovered that the detection of the tight junction protein claudin-3 in the urine signifies the breakdown of intestinal tight junctions. Therefore, the urinary excretion of claudin-3 was studied in neonates in whom NEC was suspected. Mean urinary claudin-3 level was statistically significant higher in neonates who eventually developed NEC (1267.0 \pm 175.6$)$, than in those with other final diagnoses (459.2 $\pm 108.1, p=0.0003)$ (figure 3B).
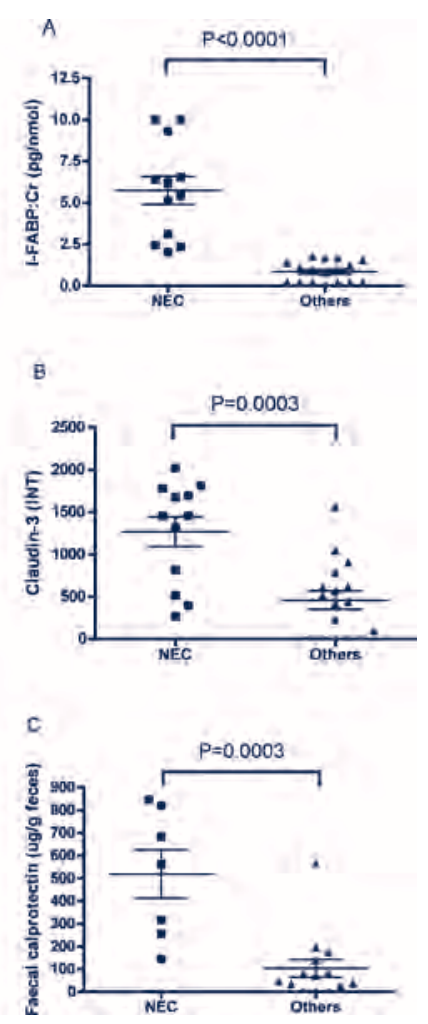

Figure 3: New non-invasive test for diagnosing NEC. A. Mean urinary I-FABP:Cr ratio was significantly higher in neonates with NEC than in neonates with other final diagnoses

B. Mean urinary claudin-3 level was significantly higher in neonates with NEC than in neonates with other final diagnoses

C. Mean faecal calprotectin level was significantly higher in neonates with NEC than in neonates with other final diagnoses 


\section{Chapter 3}

The area under the curve for claudin-3 in the diagnosis of NEC was 0.84 $(95 \% \mathrm{Cl}=0.69-0.99, \mathrm{p}=0.002)$.

\section{Faecal calprotectin levels was significantly higher in patients with NEC}

The mean calprotectin level was statistically significant higher in neonates who eventually developed NEC $(519.3 \pm 106.7)$, than in those with other final diagnoses (103.9 $\pm 39.29, \mathrm{p}=0.0003)$ (figure $3 \mathrm{C}$ ). One outliers was found in the group of patients with other diagnoses than NEC. The patient with the highest faecal calprotectin level in this group was a patient with a fulminate sepsis.

The area under the curve for faecal calprotectin in the diagnosis of NEC was $0.94(95 \% \mathrm{Cl}=0.84-1.04, \mathrm{p}=0.001)$.

\section{Discussion}

Early and correct diagnosis for NEC is crucial at the one hand to prevent delay in diagnosis of NEC and consequent morbidity and mortality, and at the other hand to avoid over treatment in neonates suspected for NEC with other final diagnosis. Neonatologists and (pediatric) surgeons rely on signs and symptoms for diagnosing NEC. Laboratory investigations (CRP, WBC, platelets) and advanced imaging techniques can help to improve diagnostic accuracy. As promising as these tests might seem, diagnostic accuracy improved only marginally in recent decades ${ }^{3,4}$. Therefore, we aimed at improving diagnostic accuracy for diagnosing NEC by using markers representing important histopathological factors; gut wall integrity loss and gut wall inflammation ${ }^{1,5}$.

Gut wall integrity loss was studied by measuring urine levels of I-FABP, indicating epithelial cell damage and claudin-3, representing tight junction loss. Faecal calprotectin was used to study gut wall inflammation. To investigate the clinical usefulness of these markers we prospectively included all neonates suspected with NEC. Our study showed that urinary I-FABP, urinary claudin-3 and faecal calprotectin were significantly higher in neonates suspected for NEC who ultimately developed NEC compared to neonates with other final diagnosis. The classical laboratory test plasma CRP also showed significantly higher levels in neonates suspected for NEC who ultimately developed NEC compared to neonates with other final diagnosis, in contrast leucocyte count and platelet count could not differentiate between the two groups. Furthermore, the overall accuracy of the markers tested was summarized using area under the curve (AUC). The AUC of I-FABP was maximum (1.0), while the AUC of calprotectin, claudin-3 and CRP were also high.

Furthermore, while previous studies have assessed the feasibility of using 
plasma I-FABP to identify patients with NEC compared with healthy controls, 8 , 9 this is the first study which prospectively identified neonates with NEC among a population of preterm infants who presented with gastrointestinal symptoms. One study showed that faecal calprotectin could differentiate between neonates with NEC compared to healthy matched controls, 5 while another prospective study showed that faecal calprotectin could not identified neonates with NEC among a population of neonates with a very low birth weight 21. Our study shows diagnostic accuracy of faecal calprotectin in the early diagnosis of NEC. This is the first study to use measurement of urinary claudin-3 in the early diagnosis of NEC.

We choose not to combine the AUCs of the markers because I-FABP already had maximum AUC, therefore it was impossible to get better results with a combination of markers. Although I-FABP resulted in a maximal distinction between the groups, we assume that measurement of the other markers might contribute to the diagnosis of NEC. In this study we included 29 neonates suspected for NEC, an increased sample size could result in altered AUCs of the markers which could necessitates combining the used markers.

All the new markers were tested in non-invasively collected material, which is of great advanced compared to the plasma tests currently in use, because blood withdrawal for diagnostics is the mean cause of anaemia in neonates $17,18$.

Given the ease and frequency with which urine and faeces can be collected without adverse consequences for the baby, the usefulness of urinary I-FABPs, claudin-3 and faecal calprotectin will be evaluated in daily clinical practice by prospective measurement of these markers in neonates suspected for NEC.

Finally, as the cumulative release of I-FABP, claudin-3 and calprotectin can be used to estimate the extent of intestinal damage, it may also help to guide treatment strategies, such as the timing of surgery (if required), the duration of antibiotic treatment, and selection of the ideal time for reintroducing enteral feeding. 


\section{Chapter 3}

\section{RefERENCES}

1. Lin PW, Stoll BJ. Necrotising enterocolitis. Lancet 2006;368:1271-83.

2. Fanaroff AA, Korones SB, Wright LL, Verter J, Poland RL, Bauer CR, Tyson JE, Philips JB, 3rd, Edwards W, Lucey JF, Catz CS, Shankaran S, Oh W. Incidence, presenting features, risk factors and significance of late onset septicemia in very low birth weight infants. The National Institute of Child Health and Human Development Neonatal Research Network. Pediatr Infect Dis J 1998;17:593-8.

3. Hallstrom M, Koivisto AM, Janas M, Tammela O. Laboratory parameters predictive of developing necrotizing enterocolitis in infants born before 33 weeks of gestation. J Pediatr Surg 2006;41:792-8.

4. Tam AL, Camberos A, Applebaum H. Surgical decision making in necrotizing enterocolitis and focal intestinal perforation: predictive value of radiologic findings. J Pediatr Surg 2002;37:1688-91.

5. Carroll D, Corfield A, Spicer R, Cairns P. Faecal calprotectin concentrations and diagnosis of necrotising enterocolitis. Lancet 2003;361:310-1.

6. Kanda T, Fujii H, Tani T, Murakami H, Suda T, Sakai Y, Ono T, Hatakeyama K. Intestinal fatty acid-binding protein is a useful diagnostic marker for mesenteric infarction in humans. Gastroenterology 1996;110:339-43.

7. Lieberman JM, Sacchettini J, Marks C, Marks WH. Human intestinal fatty acid binding protein: report of an assay with studies in normal volunteers and intestinal ischemia. Surgery 1997;121:335-42.

8. Edelson MB, Sonnino RE, Bagwell CE, Lieberman JM, Marks WH, Rozycki HJ. Plasma intestinal fatty acid binding protein in neonates with necrotizing enterocolitis: a pilot study. J Pediatr Surg 1999;34:1453-7.

9. Guthmann F, Borchers T, Wolfrum C, Wustrack T, Bartholomaus S, Spener F. Plasma concentration of intestinal- and liver-FABP in neonates suffering from necrotizing enterocolitis and in healthy preterm neonates. Mol Cell Biochem 2002;239:227-34.

10. van de Poll MC, Derikx JP, Buurman WA, Peters WH, Roelofs HM, Wigmore SJ, Dejong $\mathrm{CH}$. Liver manipulation causes hepatocyte injury and precedes systemic inflammation in patients undergoing liver resection. World J Surg 2007;31:2033-8.

11. Rahner C, Mitic LL, Anderson JM. Heterogeneity in expression and subcellular localization of claudins 2, 3, 4, and 5 in the rat liver, pancreas, and gut. Gastroenterology 2001;120:411-22.

12. Turksen K, Troy TC. Barriers built on claudins. J Cell Sci 2004;117:2435-47.

13. Zeissig S, Burgel N, Gunzel D, Richter J, Mankertz J, Wahnschaffe U, Kroesen AJ, Zeitz M, Fromm M, Schulzke JD. Changes in expression and distribution of claudin 2, 5 and 8 lead to discontinuous tight junctions and barrier dysfunction in active Crohn's disease. Gut 2007;56:61-72. 
14. D'Inca R, Dal Pont E, Di Leo V, Ferronato A, Fries W, Vettorato MG, Martines D, Sturniolo GC. Calprotectin and lactoferrin in the assessment of intestinal inflammation and organic disease. Int J Colorectal Dis 2007;22:429-37.

15. Fagerhol MK. Calprotectin, a faecal marker of organic gastrointestinal abnormality. Lancet 2000;356:1783-4.

16. Tibble J, Sigthorsson G, Foster R, Sherwood R, Fagerhol M, Bjarnason I. Faecal calprotectin and faecal occult blood tests in the diagnosis of colorectal carcinoma and adenoma. Gut 2001;49:402-8.

17. Lin JC, Strauss RG, Kulhavy JC, Johnson KJ, Zimmerman MB, Cress GA, Connolly NW, Widness JA. Phlebotomy overdraw in the neonatal intensive care nursery. Pediatrics 2000;106:E19.

18. Smoller BR, Kruskall MS. Phlebotomy for diagnostic laboratory tests in adults. Pattern of use and effect on transfusion requirements. N Engl J Med 1986;314:1233-5.

19. van der Sluijs Veer G, van den Hoven B, Russel MG, van den Bergh FA. Time-resolved fluorimetric immunoassay of calprotectin: technical and clinical aspects in diagnosis of inflammatory bowel diseases. Clin Chem Lab Med 2006;44:292-8.

20. Bossuyt PM, Reitsma JB, Bruns DE, Gatsonis CA, Glasziou PP, Irwig LM, Moher D, Rennie D, de Vet HC, Lijmer JG. The STARD statement for reporting studies of diagnostic accuracy: explanation and elaboration. Clin Chem 2003;49:7-18.

21. Josefsson S, Bunn SK, Domellof M. Fecal calprotectin in very low birth weight infants. J Pediatr Gastroenterol Nutr 2007;44:407-13. 


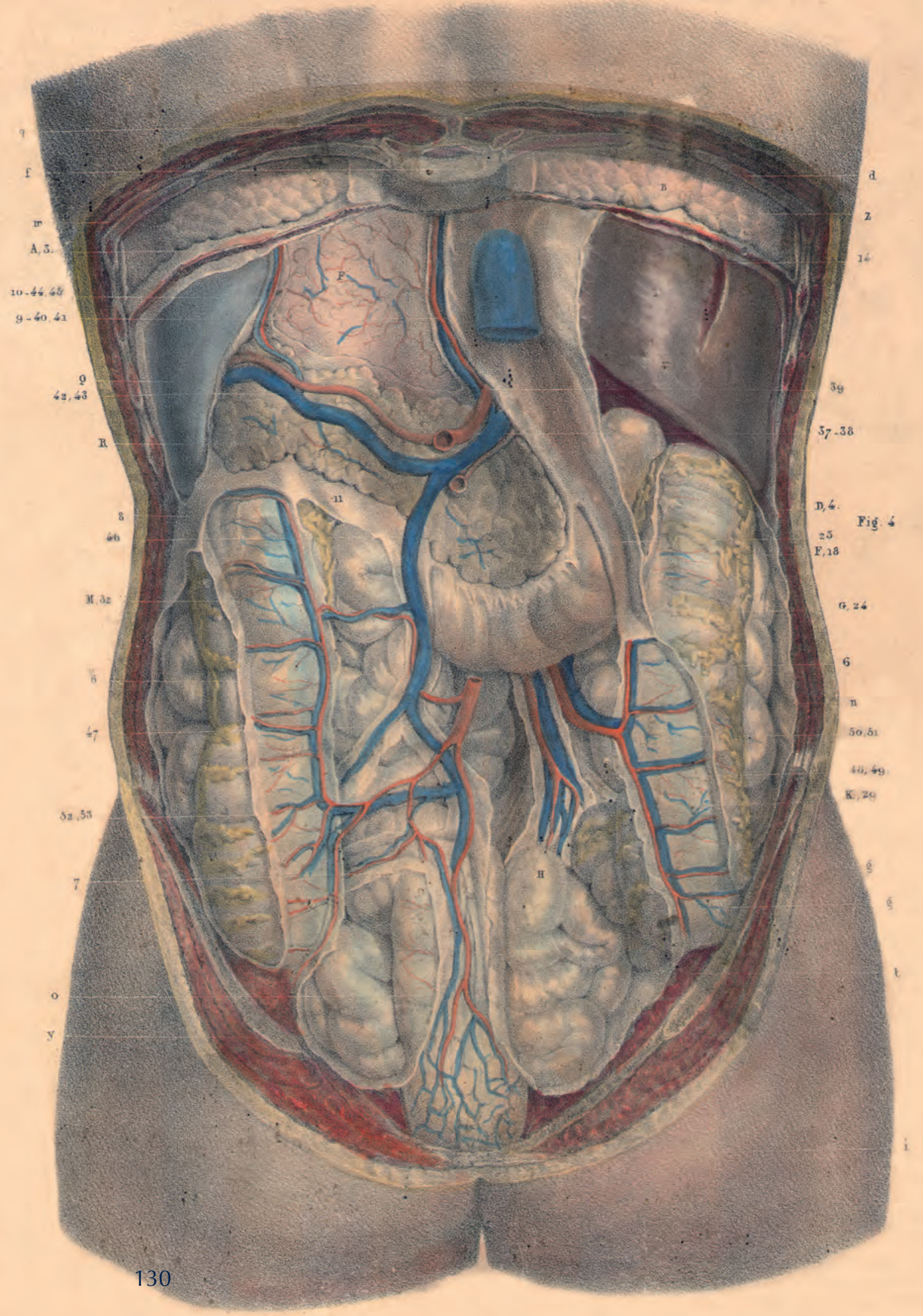




\section{Chapter 4}

Gut wall integrity loss in critically ill patients and in patients undergoing major (non-abdominal) surgery 


\section{Chapter 4}

\subsection{INTRODUCTION}

To study the third aim: investigate the integrity of the gut wall in critically ill patients and in patients undergoing major (non-abdominal) surgery, the gut wall integrity was analyzed in 4 clinical studies. First, the development of intestinal damage and its correlation with splanchnic perfusion was investigated in postsurgical critically ill patients (paragraph 4.2). In the second study, the occurrence of intestinal epithelial cell damage was investigated in critically ill patients with meningococcal sepsis, a different primary source of sepsis (paragraph 4.3). Third, the development of gut wall integrity loss was assessed peri-operatively in patients undergoing major (non-abdominal) surgery, i.e. repair of a thoraco-abdominal aneurysm of the aorta and spinal fusion repair (paragraph 4.4 and 4.5, respectively). Two of the most extensive and complex surgical procedures are open surgical repair of thoracic aortic aneurysms (TAA) and thoracoabdominal aortic aneurysms (TAAA) that are associated with significant postoperative morbidity and mortality. In order to prevent peroperative ischemic injury of visceral organs, extracorporeal circulation (ECC) technique has been implemented in open TAA repair to provide (retrograde) distal aortic perfusion (DAP) via the femoral artery. In case of TAAA repair involving the origin of visceral arteries, ECC with DAP is combined with selective organ perfusion catheters (DAP and SP) to provide designated visceral arteries directly with blood. Finally, the development of gut wall integrity loss was studied in patients undergoing major spinal fusion surgery, which is characterized by long operation time, significant blood loss, prolonged systemic hypotension and the potential development of postoperative complications. This type of surgery was chosen because it does not directly compromise the intestines by intestinal manipulation or the use of extracorporeal circulation. 


\subsection{Evidence for intestinal epithelial and liver cell injury in the early phase of sepsis}

Joep P. M. Derikx, Martijn Poeze, Annemarie A. van Bijnen, Wim A. Buurman, Erik Heineman

Shock (2007) 28: 544-8. 


\title{
Chapter 4
}

\begin{abstract}
The development of sepsis and multiple organ failure (MOF) are important determinants of the outcome in critically ill patients. Hepatosplanchnic hypoperfusion and resulting intestinal and hepatic cell damage have been implicated as central events in the development of sepsis and MOF. Our aim was to study (1) the relation between intramucosal perfusion and intestinal and hepatic cell damage in an early phase of sepsis, (2) the correlation of these parameters with mortality. Two groups of patients were consecutively selected after ICU admission: patients with postoperative abdominal sepsis $(n=19)$ and patients with pneumonia-induced sepsis $(n=9)$. Intramucosal perfusion was assessed by gastric tonometry (Pr-aCO2-gap, PiCO2). Circulating levels of Intestinal Fatty Acid Binding Protein (I-FABP) and Liver (L)-FABP were used as markers for respectively intestinal and hepatic cellular damage. Outcome was determined on day 28. Pr-aCO2-gap correlated with I-FABP (Pearson's r2=0.56, $\mathrm{p}<0.001)$ in all patients and gastric mucosal PiCO2 correlated significantly with I-FABP ( $\mathrm{r} 2=0.57, \mathrm{p}=0.001)$ in patients with abdominal sepsis. At ICU admission, non-survivors had significantly higher I-FABP and L-FABP values than survivors (I-FABP: 325 vs. 76 pg/ml, p<0.04; L-FABP: 104 vs. 31 ng/ml, $\mathrm{p}<0.04)$. Especially patients with abdominal sepsis were responsible for high admission I-FABP and L-FABP levels in non-survivors (I-FABP: 405 vs. 85 pg/ml, $\mathrm{p}<0.04$; L-FABP: $121 \mathrm{vs.} 59 \mathrm{ng} / \mathrm{ml}, \mathrm{p}<0.04)$. This study shows that splanchnic hypoperfusion correlates with intestinal mucosal damage and that elevated plasma levels of I-FABP and L-FABP are associated with a poor outcome in critically ill patients with abdominal sepsis.
\end{abstract}




\section{INTRODUCTION}

Patients with systemic inflammatory response syndrome (SIRS) and sepsis have an increased risk of developing multiple organ failure (MOF). Patients with these clinical syndromes have the highest non-cardiac mortality rate of patients in the intensive care unit (ICU) ${ }^{1}$. Recognition of patients at risk of developing MOF is therefore important. Especially patients undergoing major surgery often develop systemic inflammatory response syndrome (SIRS) ${ }^{2-4}$. Pathophysiological phenomena in the hepatosplanchnic area as compromised hepatosplanchnic perfusion leading to intestinal and hepatic damage have been widely postulated to play a central role in the transition of SIRS and sepsis into MOF ${ }^{5-9}$. Such a cellular damage causes release of constitutive hepatic and intestinal proteins in the circulation, which can act as "danger signals" and activate the immune system, resulting in the amplification of a deleterious systemic inflammatory response, which subsequently is responsible for MOF ${ }^{10}$.

In this study, two of the above mentioned key factors of this hypothesis have been objectivated clinically. Firstly, mucosal hypoperfusion was assessed by gastric tonometry ${ }^{11}$, an invasive measurement of increased tissue $\mathrm{CO} 2$ production, accompanying hypoperfusion ${ }^{12}$. Secondly, cell damage of intestinal and hepatic origin was recorded as plasma levels of Intestinal Fatty Acid Binding Protein (I-FABP) and Liver-FABP (L-FABP). I-FABP is a plasma marker for early intestinal epithelial cell damage as occurs during gut ischemia reperfusion injury, chemical epithelial cell toxicity and intestinal transplant rejection ${ }^{13,14}$. The small (14-15 kDa) cytosolic I-FABP is solely present in mature enterocytes of small and large intestine and released as soon as the cell membrane integrity is compromised and appears rapidly in the circulation after intestinal epithelial cell damage ${ }^{13}$. L-FABP is present in low amounts in mature enterocytes of small and large intestine and in abundance in the liver ${ }^{15}$.

The aim of this preliminary prospective cohort study is to investigate the relation between intramucosal oxygenation and perfusion deficits and intestinal and liver damage, and mortality in critically ill patients with an early phase of sepsis. 


\section{Chapter 4}

\section{MATERIALS AND METHODS}

\section{Patients}

After approval of the study by the Ethical Committee, patients were consecutively included after written informed consent by the next of kin. Patients were included in the study during the first $24 \mathrm{hrs}$ after admission to the intensive care unit.

Two groups of septic patients were prospectively identified. Firstly, a highrisk surgical patient group with a presumed impaired splanchnic perfusion caused by postoperative peritonitis: the abdominal sepsis group. These patients had four-quadrant peritonitis based upon intestinal leaks after primary abdominal procedures. Secondly, a group without presumed impaired splanchnic perfusion included patients with a non-abdominal derived sepsis caused by pneumonia: the pneumonia-induced sepsis group. Both abdominal and pneumonia-derived sepsis were primary reason for admission to the ICU. The selection of these groups was made prior to the analysis of blood samples. All patients suffered from septic shock, defined by systemic inflammatory response syndrome (SIRS), for which two or more of the following criteria must be positive: (1) tempe $\neg$ rature $>38^{\circ}$ or $<36^{\circ} \mathrm{C}$; (2) heart rate $>90 \mathrm{bpm}$; (3) respiratory rate $>20$ breaths per minute or $\mathrm{PaCO} 2<32 \mathrm{mmHg}$; (4) white blood cell count $>12,000 \mathrm{~mm}-3,<4,000 \mathrm{~mm}-3$, or $>10 \%$ immature (band) forms, plus infection and hypotension despite adequate fluid resuscitation. Pneumonia was defined by an infiltrate on chest radiograph, signs of SIRS and/or at least one of the following: (1) positive quantitative culture of a sample obtained by bronchoalveolar lavage $(>104 \mathrm{cfu} / \mathrm{ml})$ or protected specimen brush $(>103$ $\mathrm{cfu} / \mathrm{ml}) ;(2)$ positive blood culture unrelated to another source and obtained within $48 \mathrm{~h}$ before and after respiratory sampling. Exclusion criteria were: age $<18$ years, uncontrollable hemorrhage, cardiogenic shock, the presence of neoplastic disease, or an underlying disease expected to be rapidly fatal.

Plasma samples were obtained at day 0-7, 14, 21 and 28 after admission to ICU.

SOFA-score, hematological profiles and blood chemistry were daily recorded. On day 28, outcome was determined by classifying each patient either as survivor or non-survivor.

Sixty-nine healthy volunteers $(M: F=40: 29)$ were recruited to establish normal values for plasma I-FABP and L-FABP. Mean age was 48 years (range: 25-65 years). 


\section{Measurements of gastric mucosal tonometry}

A gastric tonometry catheter (14F, Datex Ohmeda, Finland) was introduced for measurement of intramucosal carbon dioxide pressure (PiCO2 in $\mathrm{kPa}$ ) throughout the ICU stay, using the gas-automated capnograph (Tonocap TC200, Datex-Ohmeda, Finland).

Gastric tonometry measurements (PiCO2, and mucosal-arterial pCO2 gap (Pr-aCO2-gap)) were measured at 10-minute intervals using gas-automated capnography at the same time-intervals as blood was collected. The pCO2 values of the blood gases were corrected for the central blood temperature measurements, using the formulas provided by the manufacturer ( $A B L 100$, Radiometer, Copenhagen). Patients were not fed enterally during the first 24 hours after admission to the ICU, and thereafter feeding was halted for 2 hours before measurement of gastric mucosal tonometry.

\section{Sample analyses}

Plasma I-FABP and L-FABP were determined using highly specific commercially available enzyme-linked immunosorbent assays (ELISA) (kindly provided by Hycult Biotechnology, Uden, the Netherlands). AST and ALT were determined by the clinical chemistry laboratory of the University Hospital Maastricht.

\section{Statistics}

Statistical analysis was performed with $\mathrm{SPSS}{ }^{\circledR}$ 11.0. Data were presented as mean \pm standard error or percentages. Patients were analyzed comparing data between the prospectively defined abdominal and pneumonia-induced sepsis group and between survivors and non-survivors. Concentra-tions of I-FABP and L-FABP were compared by Mann-Whitney's $U$ test. Correlations were calculated using Pearson's correlation coefficient after assessment of normal distribution. A p-value below 0.05 was considered to be statistically significant.

\section{RESULTS}

\section{Patients/demographics}

In total, 28 patients with an early phase of sepsis were recruited (Table 1); 17 patients had proven bacterial etiology. In total 19 patients with abdominal sepsis and 9 patients with pneumonia-induced sepsis were included. Of the 19 patients with abdominal sepsis, 8 died because of MOF after a median period of 4 days. Of the 9 patients with pneumonia, 4 died because of MOF after a median period of 5 days. There was no difference in both subgroups with abdominal and nonabdominal sepsis in outcome $(\mathrm{p}=0.5)$, age, gender and SOFA-score. 


\section{Chapter 4}

Table 1: Patient characteristics

\begin{tabular}{|c|c|c|c|c|}
\hline Variables & Total group & Survivors & Non-survivors & P-value \\
\hline Number & 28 & 16 & 12 & \\
\hline Age (yrs) & $64 \pm 13$ & $60 \pm 12$ & $67 \pm 14$ & 0.1 \\
\hline Gender ( $\%$ Female) & 39 & 42 & 38 & 0.7 \\
\hline Culture positive sepsis & $17 / 28$ & $6 / 1611 / 120.2$ & & \\
\hline Candida & & 0 & 3 & \\
\hline E. coli & & 2 & 3 & \\
\hline Enterobacter & & 0 & 1 & \\
\hline Enetrococcus & & 2 & 0 & \\
\hline Klebsiella & & 0 & 1 & \\
\hline Proteus & & 1 & 0 & \\
\hline Pseudomonas & & 1 & 3 & \\
\hline Abdominal & vs. 19 vs. 9 & 11 vs. 5 & 8 vs. 4 & 0.7 \\
\hline \multicolumn{5}{|l|}{ pneumonia-induced } \\
\hline \multicolumn{5}{|l|}{ sepsis } \\
\hline SOFA-score & $8.5 \pm 0.7$ & $7.9 \pm 2.9$ & $9.0 \pm 3.5$ & 0.4 \\
\hline
\end{tabular}

\section{Gastric mucosal hypoperfusion correlates with intestinal mucosal damage in patients with abdominal sepsis}

First, we investigated the proposed correlation between hepatosplanchnic hypoperfusion and intestinal and hepatic damage. To this end we studied the relation between gastric mucosal perfusion and circulatory levels of I-FABP and L-FABP.

In our patients with sepsis $\mathrm{Pr}$-aCO2-gap correlated significantly with plasma concentrations of I-FABP (Pearson's $r 2=0.56, p<0.001$ ). Gastric mucosal $\mathrm{PiCO} 2$ correlated also significantly with plasma concentrations of I-FABP $(\mathrm{r} 2=0.57, \mathrm{p}=0.001$, Figure 1$)$ in patients with abdominal sepsis. There was a weak, non-significant correlation between gastric mucosal PiCO2 and plasma I-FABP in patients with pneumonia-induced sepsis (Pearson's $r 2=0.48, p=0.08$ ). Gastric mucosal PiCO2 or Pr-aCO2-gap and plasma L-FABP did not correlate in either of the patient groups with sepsis (Pearson's $\mathrm{r} 2=0.1, \mathrm{p}=0.4$ ).

These data show a direct relationship between gastric mucosal $\mathrm{PiCO} 2$, a measure for splanchnic hypoperfusion and plasma I-FABP, a marker for intestinal mucosal damage. 


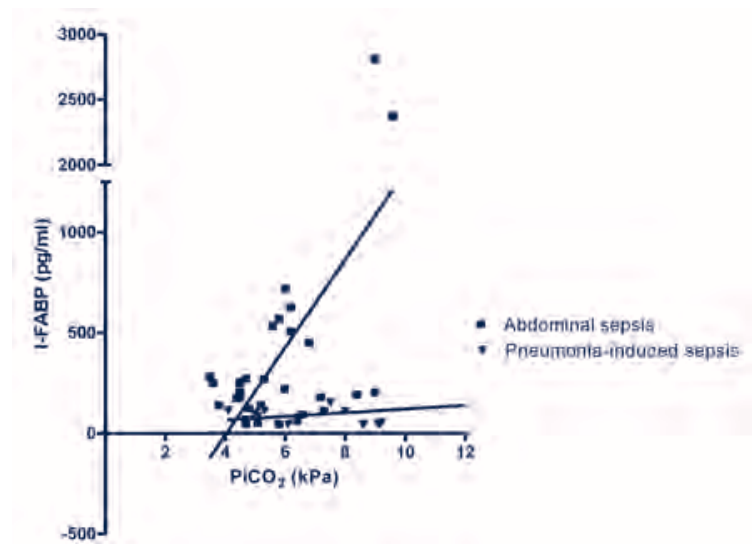

Figure 1: Correlation between splanchnic hypoperfusion $\left(\mathrm{PiCO}_{2}\right)$ and plasma levels of intestinal mucosal cell damage (circulating I-FABP) at the same time point. Circulating I-FABP correlated significantly with gastric mucosal $\mathrm{PiCO}_{2}\left(\mathrm{r}^{2}=0.57 ; \mathrm{P}=0.001\right)$ in patients with abdominal sepsis. There is no correlation between plasma valeus of I-FABP and gastric mucosal $\mathrm{PiCO}_{2}\left(\mathrm{r}^{2}=0.48 ; \mathrm{P}\right.$ $=0.08$ ) in patients with pneumonia-induced sepsis.

\section{Mucosal hypoperfusion and circulating levels of markers for intestinal and hepatic damage correlate with outcome}

Gastric mucosal PiCO2 and circulating values of I-FABP and L-FABP were compared between abdominal and pneumonia-induced sepsis and between survivors and non-survivors. Gastric mucosal PiCO2 and Pr-aCO2-gap at ICU admission were significantly higher in non-surviving patients $(p=0.04$ and $\mathrm{p}<0.05$, resp.). Moreover, non-surviving patients with abdominal sepsis had significant higher PiCO2 values than surviving patients with abdominal sepsis $(p=0.001)$ (data not shown).

The highest plasma values of both I-FABP and L-FABP were measured directly at ICU admission. After stabilization and initiation of treatment in the ICU a rapid decline of FABP-levels was seen in most patients (Figure 2). Nonsurvivors had significant higher admission values of both circulating FABPs than survivors (I-FABP: 325 vs. 76 pg/ml, p<0.04; L-FABP: 104 vs. 31 ng/ml, p<0.04, Figure 3$)$. Interestingly, especially critically ill patients with abdominal sepsis showed high levels of FABPs on admission in non-survivors compared to survivors: I-FABP: 405 vs. 85 pg/ml, p<0.04; L-FABP: 121 vs. 59 ng/ml, p<0.04. Comparison of plasma values of I-FABP and L-FABP from 69 healthy volunteers (I-FABP: $106 \pm 10 \mathrm{pg} / \mathrm{ml}$; L-FABP: $19 \pm 11 \mathrm{ng} / \mathrm{ml}$ ) with patients with pneumonia-induced sepsis (I-FABP: $91 \pm 21 \mathrm{pg} / \mathrm{ml}, \mathrm{p}=0.851$; L-FABP: $43 \pm 11$ $\mathrm{ng} / \mathrm{ml}, \mathrm{p}=0.096)$, showed that intestinal mucosal and hepatic cellular damage 


\section{Chapter 4}

was not significantly enhanced in the early phase of patients with pneumoniainduced sepsis.

Since L-FABP is primarily present in the liver and to a much lower degree also in intestine and kidney, the site of release of L-FABP was assessed by correlating L-FABP with known markers of hepatic cellular damage AST and ALT and with the intestinal mucosal damage marker I-FABP in patients with maximal increased levels of FABPs. All values of plasma L-FABP correlated significantly with plasma AST (Pearson's $r 2=0.97, \mathrm{p}<0.001$ ) and plasma ALT (Pearson's $\mathrm{r} 2=0.87, \mathrm{p}<0.001$ ), while circulating L-FABP and I-FABP did not correlate (Pearson's $r 2=0.23, p=0.2$ ). These data strongly suggest that plasma $\mathrm{L}-$ FABP represents primarily the presence of specific liver injury, while I-FABP reflects intestinal damage.
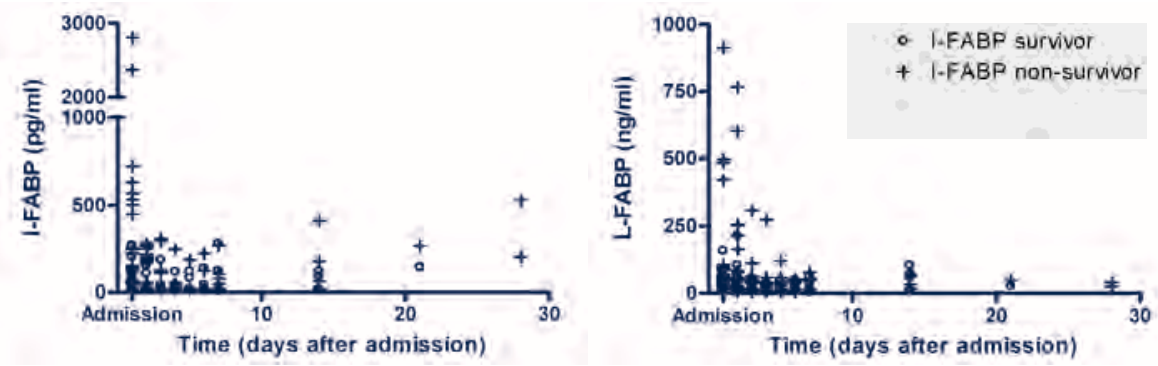

Figure 2: Time course of I-FABP (A) and L-FABP (B) plasma levels. The highest plasma levels of IFABP (A) and L-FABP (B) were measured directly after ICU admission.

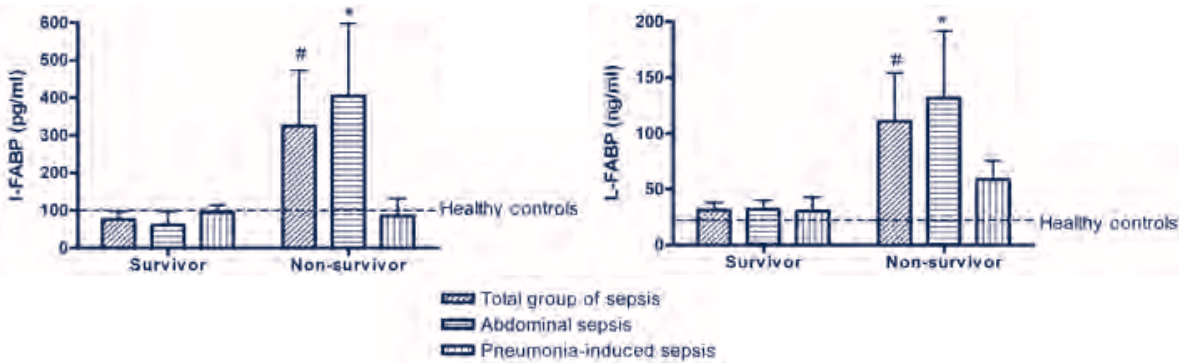

Figure 3: Admission I-FABP (A) and L-FABP (B) plasma valeus and survival in abdominal and pneumonia-induced septic patients. At the time of admission, nonsurvivors had significantly higher I-FABP (A) and L-FABP (B) valeus than survivors. Critically ill patients with abdominal sepsis were especially responsible for high admission levels of I-FABP (A) and L-FABP (B) in nonsurvivors. \# survivors vs. nonsurvivors, $\mathrm{P}<0.04$. *Abdominal vs. pneumonia-induced sepsis, $\mathrm{P}<0.04$. Broken lines give normal plasma valeus of I-FABP and L-FABP. 


\section{Discussion}

This study investigates the association of hepatosplanchnic hypoperfusion, objectivated by gastric mucosal PiCO2 11, 12, with intestinal and hepatic cell damage by measurement of plasma I-FABP and L-FABP ${ }^{13-15}$ in critically ill patients. Subsequently, the putative association of mucosal hypoperfusion and of intestinal and hepatic damage with the outcome of sepsis was studied. We report that splanchnic hypoperfusion (gastric mucosal PiCO2) in the early phase of abdominal sepsis correlates strongly with intestinal mucosal damage (plasma I-FABP). The fact that PiCO2 does not correlate well with plasma levels of I-FABP in patients with pneumonia-induced sepsis is unclear. Because of the rapid clearance of FABPs, enhanced plasma levels reflect almost directly intestinal and hepatic epithelial cell death. This study shows that elevated plasma I-FABP and L-FABP are associated with a poor outcome in septic patients and especially in patients with abdominal sepsis. This is consistent with the hypothesis that intestinal and hepatic damage caused by hypoperfusion leads to the amplification of the systemic inflammatory response during sepsis resulting in MOF and increased mortality. Further conformation is however needed to proof the hypothesis. In line with our results, assessment of FABPs can be used in further studies to unravel the pathophysiology that underlies sepsis. Moreover, if FABPs could be established as reliable or pathognomonic early indicators for intestinal and hepatic cell damage, these markers may provide new clinical diagnostic and predictive markers for outcome in septic patients. It is interesting to note that in patients with pneumonia-induced sepsis mucosal hypoperfusion and intestinal cell damage occurred to a lesser degree and lacked a relation with outcome.

Our work is supported by earlier studies reporting intraoperative splanchnic hypoperfusion or release of I-FABP separately in patients with sepsis 16-18, and finding mucosal acidosis and raised plasma I-FABP to be predictive for mortality in critically ill high-risk surgical patients ${ }^{19,20}$. Previous studies using gastric tonometry to evaluate splanchnic hypoperfusion, mainly used gastric intramucosal $\mathrm{pH}(\mathrm{pHi})$ to evaluate splanchnic hypoperfusion ${ }^{16,19}$. The present study reports the direct indices $\mathrm{PiCO} 2$ and $\mathrm{Pr}$-aCO2-gap, which are recently consented to be most specific, clinical available, parameters for splanchnic perfusion ${ }^{21}$. Our data are in line with studies showing that disturbances in PiCO2 and Pr-aCO2-gap are determinants for outcome in critically ill patients, especially in patients with abdominal sepsis. It should be mentioned however that others challenged this conclusion ${ }^{22,23}$. The heterogeneity of groups and thus the heterogeneity in pathophysiology could underlie the difference in 


\section{Chapter 4}

results. In addition however, this study is the first to describe a correlation between mucosal perfusion and oxygen defects and enhanced circulating IFABP, intestinal damage. It is not easily proven that intestinal cell death as detected with plasma I-FABP is a direct result of mucosal perfusion deficiency. This assumption is however strongly strengthened by data showing that intestinal ischemia reperfusion leads to rapid I-FABP release ${ }^{13,24,25}$.

The role of intestinal compromise in sepsis has been studied using markers for plasma levels of endotoxin and gut wall integrity. Sugar absorption tests and circulating $\lg \mathrm{M}$ and $\lg \mathrm{G}$ anti-endotoxin antibodies as markers for gut mucosal barrier loss, demonstrated that gut barrier dysfunction was associated with major surgery. However, no difference in these measurements between septic and nonseptic patients was reported ${ }^{26}$. We consider that such tests for gut integrity reflect different, most likely later phases in the development of gut compromise in high-risk surgical patients than phenomena of hypoperfusion and epithelial cell death which can be assessed by gastric tonometry and circulating FABPs.

The reduced mucosal perfusion and the proposed resulting intestinal and hepatic cellular damage is most likely caused by the circulatory perturbation in sepsis. The fact that FABP levels normalized after admission to ICU strongly supports the importance of vasocontrol treatment in such patients. We consider that the observed intestinal cellular damage is involved in the enhancement and prolongation of sepsis leading to a poor outcome.

\section{RefERENCES}

1. Angus DC, Linde-Zwirble WT, Lidicker J, Clermont G, Carcillo J, Pinsky MR: Epidemiology of severe sepsis in the United States: analysis of incidence, outcome, and associated costs of care. Crit Care Med 29:1303-1310, 2001.

2. Bown MJ, Nicholson ML, Bell PR, Sayers RD: The systemic inflammatory response syndrome, organ failure, and mortality after abdominal aortic aneurysm repair. I Vasc Surg 37:600-606, 2003.

3. Mokart D, Capo C, Blache JL, Delpero JR, Houvenaeghel G, Martin C, Mege JL: Early postoperative compensatory anti-inflammatory response syndrome is associated with septic complications after major surgical trauma in patients with cancer. Br J Surg 89:1450-1456, 2002.

4. Luyer MD, Buurman WA, Hadfoune M, Jacobs JA, Konstantinov SR, Dejong CH, Greve JW: Pretreatment with high-fat enteral nutrition reduces endotoxin and tumor necrosis factor-alpha and preserves gut barrier function early after hemorrhagic shock. Shock 21:65-71, 2004. 
5. Jones AE, Aborn LS, Kline JA: Severity of emergency department hypotension predicts adverse hospital outcome. Shock 22:410-414, 2004.

6. MacFie J, O'Boyle C, Mitchell CJ, Buckley PM, Johnstone D, Sudworth P: Gut origin of sepsis: a prospective study investigating associations between bacterial translocation, gastric microflora, and septic morbidity. Gut 45:223-228, 1999.

7. Poeze M, Ramsay G, Buurman WA, Greve JW, Dentener M, Takala J: Increased hepatosplanchnic inflammation precedes the development of organ dysfunction after elective high-risk surgery. Shock 17:451-458, 2002.

8. Tamion F, Richard V, Sauger F, Menard JF, Girault C, Richard JC, Thuillez C, Leroy J, Bonmarchand G: Gastric mucosal acidosis and cytokine release in patients with septic shock. Crit Care Med 31:2137-43, 2003.

9. Backstrom T, Liska J, Oldner A, Lockowandt U, Franco-Cereceda A: Splanchnic metabolism during gut ischemia and short-term endotoxin and hemorrhagic shock as evaluated by intravasal microdialysis. Shock 21:572-578, 2004.

10. Matzinger P: The danger model: a renewed sense of self. Science 296:301-305, 2002.

11. Elizalde JI, Hernandez C, Llach J, Monton C, Bordas JM, Pique JM, Torres A: Gastric intramucosal acidosis in mechanically ventilated patients: role of mucosal blood flow. Crit Care Med 26:827-832, 1998.

12. Cerny V, Cvachovec K: Gastric tonometry and intramucosal pH--theoretical principles and clinical application. Physiol Res 49:289-297, 2000.

13. Lieberman JM, Sacchettini J, Marks C, Marks WH: Human intestinal fatty acid binding protein: report of an assay with studies in normal volunteers and intestinal ischemia. Surgery 121:335-342, 1997.

14. Pelsers MM, Hermens WT, Glatz JF: Fatty acid-binding proteins as plasma markers of tissue injury. Clin Chim Acta 352:15-35, 2005.

15. Monbaliu D, de Vries B, Crabbe T, van Heurn E, Verwaest C, Roskams T, Fevery J, Pirenne J, Buurman WA: Liver fatty acid-binding protein: an early and sensitive plasma marker of hepatocellular damage and a reliable predictor of graft viability after liver transplantation from non-heart-beating donors. Transplant Proc 37:413-416, 2005.

16. Holland J, Carey M, Hughes N, Sweeney K, Byrne PJ, Healy M, Ravi N, Reynolds JV: Intraoperative splanchnic hypoperfusion, increased intestinal permeability, downregulation of monocyte class II major histocompatibility complex expression, exaggerated acute phase response, and sepsis. Am J Surg 190:393-400, 2005.

17. Lieberman JM, Marks WH, Cohn S, Jaicks R, Woode L, Sacchettini J, Fischer B, Moller B, Burns G: Organ failure, infection, and the systemic inflammatory response syndrome are associated with elevated levels of urinary intestinal fatty acid binding protein: study of 100 consecutive patients in a surgical intensive care unit. J Trauma 45:900-906, 1998.

18. Gollin G, Zieg PM, Cohn SM, Lieberman JM, Marks WH: Intestinal mucosal injury in critically ill surgical patients: preliminary observations. Am Surg 65:19-21, 1999. 


\section{Chapter 4}

19. Poeze M, Takala J, Greve JW, Ramsay G: Pre-operative tonometry is predictive for mortality and morbidity in high-risk surgical patients. Intensive Care Med 26:12721281, 2000.

20. Levy B, Gawalkiewicz P, Vallet B, Briancon S, Nace L, Bollaert PE: Gastric capnometry with air-automated tonometry predicts outcome in critically ill patients. Crit Care Med 31:474-480, 2003.

21. Chapman MV, Mythen MG, Webb AR, Vincent JL: Report from the meeting: Gastrointestinal Tonometry: State of the Art. 22nd-23rd May 1998, London, UK. Intensive Care Med 26:613-622, 2000.

22. Gomersall CD, Joynt GM, Ho KM, Young RJ, Buckley TA, Oh TE: Gastric tonometry and prediction of outcome in the critically ill. Arterial to intramucosal $\mathrm{pH}$ gradient and carbon dioxide gradient. Anaesthesia 52:619-623, 1997.

23. Knotzer H, Pajk W, Dunser MW, Maier S, Mayr AJ, Ritsch N, Friesenecker B, Hasibeder WR: Regional microvascular function and vascular reactivity in patients with different degrees of multiple organ dysfunction syndrome. Anesth Analg 102:1187-1193, 2006.

24. Gollin G, Marks C, Marks WH: Intestinal fatty acid binding protein in serum and urine reflects early ischemic injury to the small bowel. Surgery 113:545-551, 1993.

25. Kanda T, Fujii H, Tani T, Murakami H, Suda T, Sakai Y, Ono T, Hatakeyama K: Intestinal fatty acid-binding protein is a useful diagnostic marker for mesenteric infarction in humans. Gastroenterology 110:339-43, 1996.

26. Kanwar S, Windsor AC, Welsh F, Barclay GR, Guillou PJ, Reynolds JV: Lack of correlation between failure of gut barrier function and septic complications after major upper gastrointestinal surgery. Ann Surg 231:88-95, 2000. 


\subsection{Gut mucosal damage in the early phase of children with meningococcal sepsis}

Joep P. M. Derikx, Else M. Bijker, Gijs D. Vos, Annemarie A. van Bijnen, Erik Heineman, Wim A. Buurman, Dick A. van Waardenburg Critical Care Medicine, in revision. 


\begin{abstract}

\section{Objective}

The pathophysiological sequelae of meningococcal sepsis (MS) are mainly caused by deregulated microvasculature function, leading to impaired tissue blood flow. Since especially mature enterocytes are known to be susceptible to altered perfusion, we aimed to investigate: (1) the development of enterocyte damage, (2) the relation between enterocyte damage and severity of disease and outcome, in children with MS.
\end{abstract}

\title{
Design
}

Retrospective human study.

\section{Setting}

Paediatric intensive care unit (PICU) at a university hospital.

\section{Patients}

19 consecutively included children with MS were studied during their PICU stay.

\section{Interventions}

None for the study.

\section{Measurement and Main results}

Circulating levels of I-FABP, a small cytosolic protein constitutively present in mature enterocytes and released upon cell injury, were assessed. Severity of disease was represented by meningococcal-specific Rotterdam Score (RS), generic Paediatric Risk of Mortality II (PRISM II) score and circulating interleukine-6 (IL-6). Clinical outcome was measured by length of PICU-stay and number of ventilator days.

Highest plasma I-FABP values were measured on PICU admission. At the time of admission, 8 of 19 patients had higher I-FABP plasma levels than the upper reference limit of 30 healthy volunteers. In all survivors I-FABP levels declined to normal values within 12 hours after starting intensive treatment, while the three non-survivors maintained elevated I-FABP plasma levels. A significant correlation was found between I-FABP and RS, PRISM II, IL-6 at admission (Spearman $r 2=0.402, p=0.006 ; r 2=0.243, p=0.045 ; r 2=0.687, p$ $<0.001$, respectively). Next, a significant correlation was found between I-FABP and clinical outcome. 


\section{Conclusions}

Elevated plasma I-FABP is found in 8 out of 19 children with an early phase of severe MS, suggesting the presence of enterocyte damage. Moreover, prolonged enterocyte damage is found in non-survivors. Further studies are needed to clarify the potential role for assessment of plasma I-FABP in monitoring treatment of MS.

\section{INTRODUCTION}

Meningococcal sepsis (MS) is one of the most severe infections in childhood. It is well known for its sudden onset and rapid progression to septic shock. Despite early use of antibiotics, aggressive haemodynamic support and lifesustaining care, the overall mortality of children with MS remains significant (2$12 \%)^{1-3}$. Furthermore, MS is associated with severe morbidity, including limb necrosis and multiple organ failure (MOF) ${ }^{2,4}$.

Dysregulation of microvasculature function is a major pathophysiological event in patients with MS ${ }^{4}$. The development of septic shock and MOF in MS is explained by a complex of phenomena, including hypovolaemia resulting from increased vascular permeability, pathological vasoconstriction and vasodilatation, loss of thromboresistance, intravascular coagulation and profound myocardial dysfunction. These processes lead to impairment of the microvascular blood flow in tissues and organs, causing ischemic injury ${ }^{4}$. Organs that are mostly affected include kidneys, lungs and splanchnic organs ${ }^{4}$.

The mature intestinal mucosal cells are especially susceptible to hypoperfusion and ischemia ${ }^{5,6}$. Damage of intestinal mucosa can lead to local inflammation, impaired barrier function, and thereupon potentially to translocation of micro-organisms ${ }^{6-9}$. This translocation can result in a derailment of local inflammation and subsequently amplification of a systemic inflammatory response, as is mainly shown in animal studies ${ }^{10,11}$. Studies in critically ill adults also indicate that intestinal damage caused by splanchnic hypoperfusion is associated with the transition of the systemic inflammatory response syndrome (SIRS) into sepsis and septic shock ${ }^{12-15}$.

Intestinal fatty acid binding protein (I-FABP) is a small (14-15kDa) cytosolic protein constitutively present in mature enterocytes and released upon cell injury ${ }^{16,17}$. I-FABP circulates in low amounts in the blood stream of healthy individuals. The levels of circulating I-FABP correlate with the extent of splanchnic hypoperfusion in adult critically ill patients ${ }^{15}$. Assessment of plasma I-FABP levels makes it possible to evaluate gut mucosal damage in disease conditions associated with redistribution of blood flow to vital organs at the expense of the splanchnic circulation. 


\section{Chapter 4}

The primary aim of this retrospective study was to investigate the presence of gut mucosal damage by (serial) assessment of plasma I-FABP levels in children with MS during the first 48 hours after admission. Our second aim was to study the relation between the presence of gut mucosal damage and severity of disease, intravascular thrombosis and clinical outcome.

\section{MATERIALS AND METHODS}

\section{Patients}

Children between the ages of 6 months and 16 years consecutively admitted to the paediatric intensive care unit (PICU) of the Maastricht University Medical Centre, between October 2006 and October 2007 were retrospectively enrolled in the study. All children fulfilled the diagnosis of MS as defined according to the sepsis criteria of the Society of Critical Care Medicine and the American College of Chest Physicians (SCCM/ACCP), adapted to infants and children (18), in combination with petechiae and/or purpura and a positive blood or spinal fluid culture with Neisseria meningitidis. All patients had an indwelling arterial catheter. Children with known abdominal disease were excluded, because such a disease could be a cause of intestinal epithelial damage and corroborate the data. All children were treated according to institutional guidelines, including aggressive treatment of respiratory and circulatory failure with mechanical ventilation, large volume intravenous fluid replacement, vaso-active and inotropic drugs (norepinephrine dopamine), and intravenous antibiotics. Continuous enteral feeding was started within the first 24 hours after admission in all patients and increased in volume during the next days according to our standard feeding protocol. Normal plasma values of I-FABP were established from thirty healthy volunteers (median age: 5.8 years, range: 1-14 years), which consisted of children that consecutively underwent minor non-abdominal surgery (e.g. removal of K-wires, circumcision). The study was approved by the local Medical Ethical Committee.

\section{Clinical parameters}

To assess severity of illness the Rotterdam score (RS) was calculated. The RS is a prognostic score validated to indicate severity of disease in children with MS ${ }^{19}$. Further, also the Paediatric Risk of Mortality II (PRISM II) score was assessed, which is a validated generic scoring system to predict risk of mortality in heterogeneous groups of paediatric ICU patients ${ }^{20}$. Disseminated intravascular coagulation (DIC) was assessed using the global coagulation test score for DIC as described by Dhainaut et al. ${ }^{21}$. Clinical outcome of the disease was assessed as length of stay in the PICU and number of days on the ventilator. 


\section{Blood analysis \\ Blood sampling}

Arterial blood samples were routinely collected directly after admission and subsequently 6, 12, 24 and 48 hours after admission for measurement of Creactive protein (CRP), creatinine, platelet count, lactate, blood gasses, creatinine and potassium among other routine measurements. The remainder of the blood was centrifuged 15 minutes at $4000 \mathrm{rpm}$ at $4^{\circ} \mathrm{C}$ to obtain plasma and stored in aliquots at $-80^{\circ} \mathrm{C}$. This leftover plasma was used in the present study for determination of I-FABP and IL-6.

\section{Laboratory determinations}

CRP, lactate, base excess, creatinine, potassium, platelet count, D-Dimer and prothrombin time were measured by the clinical chemistry or haematological laboratory of the Maastricht University Medical Centre. Plasma I-FABP was determined by using a highly specific commercially available enzyme-linked immunosorbent assay (ELISA) that selectively detects human I-FABP (standard: 20$5,000 \mathrm{pg} / \mathrm{ml}$, kindly provided by Hycult Biotechnology, Uden, the Netherlands). IL-6 was determined using an in-house ELISA as described previously ${ }^{22}$. The detection limit of IL-6 was $0.01 \mathrm{ng} / \mathrm{ml}$.

\section{Statistical analysis}

Statistical analysis was performed with GraphPad PRISM 4.0 for Windows ${ }^{\circledR}$ (GraphPad Software Inc. San Diego, CA). Normality of all data was verified by Kolmogorov-Smirnov test. The plasma values of I-FABP were compared between patients at time of admission and healthy children using unpaired $t$ test. Friedman two-way analysis of variance by ranks was used to analyze changes in plasma I-FABP levels of MS patients between different time points. A post-hoc Dunn's multiple comparison test was used to calculate significant differences of plasma values of I-FABP with respect to baseline $(t=0)$. Correlations between plasma I-FABP levels at admission and RS, PRISM II, circulating IL-6, plasma creatinine, DIC-score, length of PICU-stay and number of ventilator days were calculated using Spearman rank correlation coefficient. Data are presented as median (range). Statistical significance was defined as $p<0.05$. 


\section{Chapter 4}

\section{RESULTS}

\section{Patients}

Nineteen patients with MS were enrolled in the study. Patient characteristics are shown in Table 1. Three patients died: one 14 hours after admission because of refractory circulatory shock (patient $A$ ), the second patient died 20 hours after admission because of brain death (patient $B$ ) and the third patient died 19 days after admission after withdrawing treatment, because of extended gut necrosis incompatible with life (patient $C$ ).

Table 1: Patient characteristics

\begin{tabular}{|c|c|}
\hline Variable $(n=19)$ & Median (range) \\
\hline Age - months & $29(11-186)$ \\
\hline Bodyweight - kg & $13(7-60)$ \\
\hline Sex (no (\%) male) & $9(50)$ \\
\hline PRISM $\|^{1}$ & $16(2-52)$ \\
\hline $\mathrm{RS}^{2}$ at admission & $-2(-8-5)$ \\
\hline Length of stay in PICU ${ }^{3}$ - days & $5(1-30)$ \\
\hline Requirement for mechanical ventilation - no. (\%) & $14(78)$ \\
\hline Ventilator days & $4(0-11)$ \\
\hline Presence of skin necrosis - no. (\%) & $7(39)$ \\
\hline Maximum dose of dopamine ( $\mu \mathrm{g} / \mathrm{kg} / \mathrm{min})$ & $20(0-20)$ \\
\hline Maximum dose of norepinephrine ( $\mu \mathrm{g} / \mathrm{kg} / \mathrm{min})$ & $0.2(0-9.5)$ \\
\hline DIC score ${ }^{4}$ & $5(1-7)$ \\
\hline \multicolumn{2}{|l|}{1 PRISM II: Pediatric Risk of Mortality II } \\
\hline \multicolumn{2}{|l|}{2 RS: Rotterdam Score } \\
\hline \multicolumn{2}{|l|}{3 PICU: paediatric intensive care unit } \\
\hline \multicolumn{2}{|c|}{${ }^{4}$ DIC: disseminated intravascular coagulation } \\
\hline
\end{tabular}

\section{Gut mucosal damage in children with meningococcal sepsis}

To investigate the occurrence of intestinal damage in the early stage of MS, circulating levels of I-FABP, representing enterocyte cell death, were determined longitudinally, at admission and subsequently at 6, 12, 24 and 48 hours after admission.

The highest plasma values of I-FABP were measured at admission on the PICU (median 298 pg/ml, range: 25-4,351 pg/ml; Figure 1). MS patients had significantly elevated I-FABP levels at the time of admission, compared to the 
thirty healthy children $(p=0.004)$, who had median circulating I-FABP values of $183(25-346) \mathrm{pg} / \mathrm{ml}$. At the time of admission, 8 of the 19 patients had higher IFABP plasma levels than the upper reference limit of the healthy volunteers (mean I-FABP $+2 *$ standard deviation $=399 \mathrm{pg} / \mathrm{ml}$ ). I-FABP plasma values declined significantly in 48 hours (Friedman $\mathrm{p}<0.01$ ). After 6 hours, only 4 patients had I-FABP levels exceeding $399 \mathrm{pg} / \mathrm{ml}$. Interestingly, in all survivors IFABP levels declined to normal values $(<399 \mathrm{pg} / \mathrm{ml})$ within 12 hours after starting intensive medical treatment, while the three non-survivors maintained elevated I-FABP plasma levels.

Since I-FABP is rapidly cleared by the kidneys, we investigated whether elevated I-FABP levels could be caused by compromised renal function. No correlation was found between plasma creatinine levels and plasma I-FABP levels at time of admission in our study group (Spearman $r^{2}=0.049, p=0.394$ data not shown). This suggests that high circulating I-FABP levels are primarily due to intestinal damage, and not to renal failure.

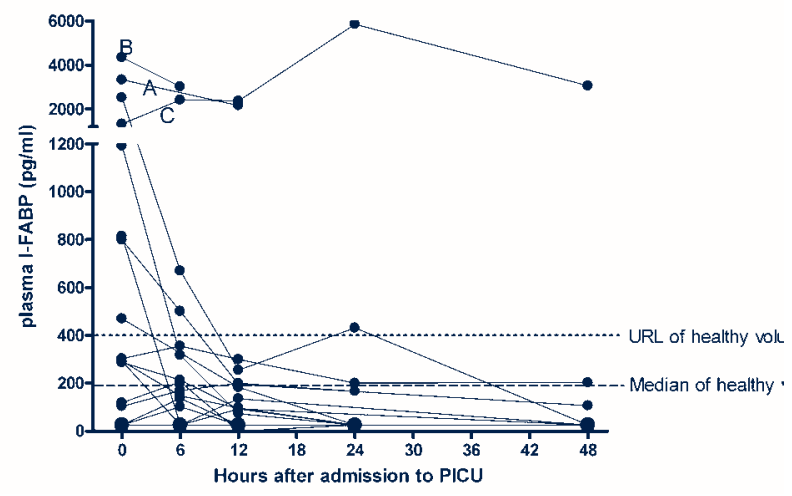

Figure 1: Plasma I-FABP values in individual patients during early phase of MS. Raised I-FABP levels were present in 8 of the 19 patients with MS compared with the upper reference limit (URL) of the 30 healthy volunteers (mean I-FABP $+2 *$ standard deviation=399 $\mathrm{pg} / \mathrm{ml}$ ). In all survivors, plasma I-FABP levels declined within 12 hours to normal; in non-survivors, however, plasma IFABP levels remained high, despite intensive treatment. Upper dotted line represents the URL of plasma I-FABP levels of 30 healthy children; lower dotted line represents the mean value . A, B, C depict the values of the three patients who died.

Our data indicate that intestinal damage is present in a significant part of the children with meningococcal sepsis in an early stage. Moreover, the persistence of high I-FABP plasma levels in non-surviving children and rapid 


\section{Chapter 4}

normalization of I-FABP values in surviving children, suggest a relationship between extent of intestinal damage and mortality.

Patient A (Figure 1) was an 11 months old girl who developed immediately after admission widespread ecchymoses, limb ischemia and necrosis, and signs of multiple organ failure. She died 14 hours after admission from refractory septic shock. Plasma I-FABP level was $3,337 \mathrm{pg} / \mathrm{ml}$ at admission and remained high $(2,164 \mathrm{pg} / \mathrm{ml}) 12$ hours after admission. Patient $B$ was an 11 months old girl who developed severe ischemia and necrosis of all four limbs and multiple organ failure within 12 hours after admission to the PICU. She developed clinical symptoms of brain death and the EEG was isoelectric. Further treatment was withheld and she died 20 hours after admission. In this girl plasma values of I-FABP were high at moment of admission $(4,352 \mathrm{pg} / \mathrm{ml})$ with continuous elevated levels at 6 hours $(3,024 \mathrm{pg} / \mathrm{ml})$. Patient $C$ was a 12 months old boy who developed severe limb ischemia and necrosis, multiple organ failure, epileptic seizures and a distended abdomen. Peritoneal dialysis was started for renal failure. After 72 hours his circulatory condition gradually improved. On day 7 he became severely septic again, while still on the ventilator and was treated for suspected peritonitis. Because of an increasingly distended abdomen and persistent metabolic acidosis, laparotomy was performed which showed complete necrosis of the small and large bowel and multiple perforations. Treatment was withheld and the patient died. I-FABP plasma level was high at admission $(1,327 \mathrm{pg} / \mathrm{ml})$ and increased towards a peak of $5,858 \mathrm{pg} / \mathrm{ml}$ at 24 hours after admission. The last collected sample, 48 hours after admission, showed an I-FABP level which was still elevated $(3,060 \mathrm{pg} / \mathrm{ml})$.

\section{The extent of gut mucosal damage is correlated with severity of disease and clinical outcome}

Next, we investigated whether the extent of intestinal damage was related to severity of disease, by correlating plasma I-FABP levels with RS, Prism II score and plasma IL-6 values, a classical sepsis parameter. A significant correlation was found between I-FABP and RS at admission (Spearman $r^{2}=0.402, p=0.006$, Figure 2A). I-FABP also correlated significantly with PRISM II score $\left(r^{2}=0.243, p=0.045\right)$. Furthermore, I-FABP plasma concentrations correlated significantly with plasma IL-6 values at admission (Spearman $\mathrm{r}^{2}=0.687, \mathrm{p}<0.001$, Figure 2B). Interestingly, all patients with plasma I-FABP levels higher than $399 \mathrm{pg} / \mathrm{ml}$ showed IL-6 levels above $100 \mathrm{pg} / \mathrm{ml}$. Next, to assess the relation between intestinal mucosal cell damage and intravascular thrombosis, the correlation was determined between plasma I-FABP levels and DIC-score at admission: I-FABP correlated significantly with DIC-score at admission (Spearman $r^{2}=0.565, p=0.005$ ). 
To study the relation between early presence of intestinal epithelial cell injury and outcome, we investigated the correlation between plasma I-FABP values at admission and number of days on the PICU and number of days at the ventilator. A significant correlation was found for both parameters $\left(r^{2}=0.457\right.$, $p=0.006$ for length of PICU-stay and $r s=0.357, p=0.019$ for number of ventilator days).

These data indicate a strong relationship between gut mucosal damage, measured by I-FABP, and severity of disease (represented by the RS, PRISM II), IL-6, DIC and clinical outcome.
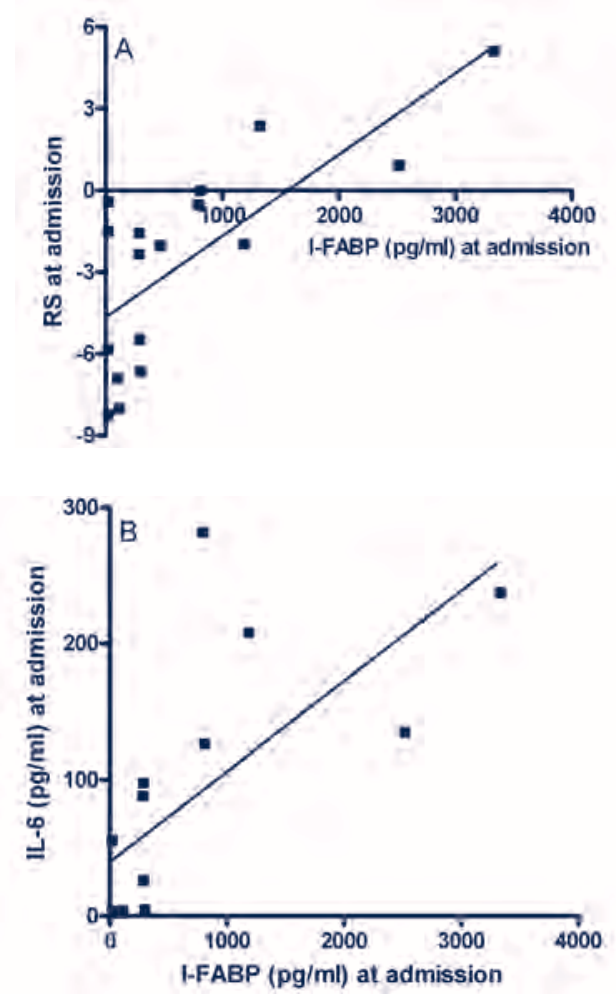

Figure 2: Correlation of intestinal epithelial cell damage (plasma I-FABP levels) and severity of disease at admission. Circulating I-FABP correlates significantly with the Rotterdam Score (RS) (A) and IL-6 (B) $\left(r^{2}=0.402, p=0.006\right.$ and $r^{2}=0.687, p<0.001$, respectively) in patients with meningococcal sepsis at time of admission to the PICU. 


\section{Chapter 4}

\section{Discussion}

The presence of intestinal epithelial cell damage in the early phase of MS in children was studied by assessment of plasma levels of I-FABP, a small protein, exclusively present in the cytosol of mature enterocytes and rapidly released upon ischemic cellular injury ${ }^{16}$. Subsequently, the correlation between intestinal damage and severity of MS, DIC and clinical outcome was investigated.

The principal findings of this study are: (1) intestinal epithelial cell damage was present in a large part (8/19) of the patients in an early phase of MS; (2) the extent of intestinal epithelial cell damage at the moment of admission to the PICU correlated with severity of disease, diffuse intravascular coagulation and clinical outcome; (3) in all survivors, plasma I-FABP levels declined to normal values within 12 hours after starting intensive medical treatment. In nonsurvivors, however, plasma I-FABP levels remained high, despite intensive treatment. Since our study group consisted only of 19 children, these results have to be confirmed in a larger population.

Primarily animal studies have provided evidence that the gut plays an important role in the transition of systemic inflammatory response (SIRS) into sepsis and multiple organ failure (MOF) ${ }^{6,9,11,12}$. SIRS potentially leads to splanchnic hypoperfusion, because blood is shifted away from the splanchnic organs towards the more vital organs (heart, brain). Especially the mature enterocytes are susceptible to hypoperfusion and will be damaged, which leads to loss of the intestinal barrier and translocation of microbiota and microbial products $5,7,8,10$. These danger signals possibly amplify the systemic inflammation by further release of cytokines, eventually resulting in a deregulated inflammatory response and MOF ${ }^{6,9,12}$. This is indeed confirmed by studies in adults and children with non-intestinal origin of sepsis, showing that increased intestinal permeability and low values of antibodies directed against the core of endotoxin (EndoCAb) are associated with the development of sepsis, multiple organ dysfunction syndrome and poor outcome ${ }^{14,23,24}$.

The presence of intestinal epithelial cell damage in our patients with more severe sepsis (as shown by high RS scores and high IL-6 levels) suggests that intestinal hypoperfusion occurs in these patients. Although there is a lack of reports on the assessment of splanchnic perfusion in children with MS, previous studies in critically ill adult patients have shown that SIRS, sepsis and shock can lead to splanchnic hypoperfusion ${ }^{15,25}$. Furthermore, splanchnic hypoperfusion is known to result in intestinal mucosal cell damage, in our study assessed by plasma levels of I-FABP ${ }^{15}$. In line, the level of circulating I-FABP has been 
reported to correlate with the histological status of the epithelium after intestinal ischemia-reperfusion ${ }^{26,27}$. In order to further unravel the role of splanchnic hypoperfusion on gut mucosal damage, it would be interesting to additionally measure gut perfusion by gastric tonometry in the acute phase of MS in children ${ }^{28}$.

The marked decrease of plasma I-FABP levels in all surviving children within 12 hours after admission to the PICU suggests that our aggressive medical treatment, targeting haemodynamic stabilization, improved intestinal perfusion, resulting in a rapid reduction of further intestinal epithelial cell damage. Besides compromised organ perfusion due to vasoregulatory disturbances, peripheral microcirculation in MS patients is also negatively affected by intravascular thrombosis. Indeed a strong correlation was found between gut mucosal damage severity of DIC in the children with MS in the present study. We speculate that uncontrolled thromboresistance and intravascular coagulation on top of splanchnic hypoperfusion may have contributed to the extreme high plasma I-FABP levels on admission in the three non-surviving MS patients and the persistence of elevated plasma I-FABP levels in spite of our attempts to restore organ perfusion by high volume fluid therapy and vaso-active and inotropic therapy.

Assessment of plasma levels of I-FABP in children with MS may have important clinical implications. The adequacy of treatment of circulatory failure in MS is currently monitored using indirect parameters of tissue perfusion and oxygenation, including blood pressure, urine output, metabolic status (lactate, base deficit) skin temperature and capillary refill time ${ }^{29}$. However, these parameters do not reflect the actual defects in (peripheral) tissue perfusion and subsequent tissue damage. Assessment of plasma I-FABP levels offers the possibility to monitor the presence of intestinal epithelial cell damage as a consequence of splanchnic hypoperfusion, which could help to monitor treatment directed at restoration of peripheral perfusion and prevention of organ damage. Additionally, the serial assessment of plasma I-FABP levels can provide important information about gut integrity since intestinal necrosis, a life-threatening complication in children with MS, may also be reflected by prolonged high I-FABP plasma levels. This is illustrated by one of the three dead children in our study, and is also in line with previous reports, showing serious intestinal complications in children with MS, including gastrointestinal perforation and haemorrhage from small bowel ulceration ${ }^{30,31}$. Early detection of intestinal necrosis may then result in timely resection of the necrotic and perforated part of the intestines. 


\section{Chapter 4}

\section{Conclusions}

Our data are the first to report the presence of intestinal epithelial cell damage in children in the early phase of severe MS. Furthermore, our data show that only in non-surviving children intestinal epithelial cell damage persisted during circulatory stabilization. Further studies are needed to clarify the diagnostic potential of assessment of plasma I-FABP in monitoring the treatment of MS in the acute phase and during follow-up.

\section{ReferenCES}

1. Rosenstein NE, Perkins BA, Stephens DS, et al. The changing epidemiology of meningococcal disease in the United States, 1992-1996. I Infect Dis 1999;180:18941901

2. Rosenstein NE, Perkins BA, Stephens DS, et al. Meningococcal disease. N Engl J Med 2001;344:1378-1388

3. Goldacre MJ, Roberts SE, Yeates D. Case fatality rates for meningococcal disease in an English population, 1963-98: database study. Bmj 2003; 327:596-597

4. Pathan N, Faust SN, Levin M. Pathophysiology of meningococcal meningitis and septicaemia. Arch Dis Child 2003;88:601-607

5. Takala J. Determinants of splanchnic blood flow. Br J Anaesth 1996;77:50-58

6. Rowlands BJ, Soong CV, Gardiner KR. The gastrointestinal tract as a barrier in sepsis. $\mathrm{Br}$ Med Bull 1999;55:196-211

7. Tamion F, Richard V, Sauger F, et al. Gastric mucosal acidosis and cytokine release in patients with septic shock. Crit Care Med 2003;31:2137-2143

8. Gatt M, Reddy BS, MacFie J. Review article: bacterial translocation in the critically ill-evidence and methods of prevention. Aliment Pharmacol Ther 2007;25:741-757

9. Fink MP, Delude RL. Epithelial barrier dysfunction: a unifying theme to explain the pathogenesis of multiple organ dysfunction at the cellular level. Crit Care Clin 2005;21:177-196

10. Yang R, Han X, Uchiyama T, et al. IL-6 is essential for development of gut barrier dysfunction after hemorrhagic shock and resuscitation in mice. Am J Physiol Gastrointest Liver Physiol 2003;285:G621-629

11. Rotstein OD. Pathogenesis of multiple organ dysfunction syndrome: gut origin, protection, and decontamination. Surg Infect (Larchmt) 2000;1:217-223

12. Stechmiller JK, Treloar D, Allen N. Gut dysfunction in critically ill patients: a review of the literature. Am J Crit Care 1997;6:204-209 
13. MacFie J, O'Boyle C, Mitchell CJ, et al. Gut origin of sepsis: a prospective study investigating associations between bacterial translocation, gastric microflora, and septic morbidity. Gut 1999;45:223-228

14. Doig CJ, Sutherland LR, Sandham JD, et al. Increased intestinal permeability is associated with the development of multiple organ dysfunction syndrome in critically ill ICU patients. Am J Respir Crit Care Med 1998;158:444-451

15. Derikx JP, Poeze M, van Bijnen AA, et al. Evidence for intestinal and liver epithelial cell injury in the early phase of sepsis. Shock 2007;28:544-548

16. Lieberman JM, Sacchettini J, Marks C, Marks WH. Human intestinal fatty acid binding protein: report of an assay with studies in normal volunteers and intestinal ischemia. Surgery 1997;121:335-342

17. Pelsers MM, Hermens WT, Glatz JF. Fatty acid-binding proteins as plasma markers of tissue injury. Clin Chim Acta 2005;352:15-35

18. Jafari HS, McCracken GH, Jr. Sepsis and septic shock: a review for clinicians. Pediatr Infect Dis / 1992;11:739-748

19. Kornelisse RF, Hazelzet JA, Hop WC, et al. Meningococcal septic shock in children: clinical and laboratory features, outcome, and development of a prognostic score. Clin Infect Dis 1997;25:640-646

20. Pollack MM, Ruttimann UE, Getson PR. Paediatric risk of mortality (PRISM) score. Crit Care Med 1988;16:1110-1116

21. Dhainaut JF, Yan SB, Joyce DE, et al. Treatment effects of drotrecogin alfa (activated) in patients with severe sepsis with or without overt disseminated intravascular coagulation. J Thromb Haemost 2004;2:1924-1933

22. Dentener MA, Bazil V, Von Asmuth EJ, et al. Involvement of CD14 in lipopolysaccharide-induced tumor necrosis factor-alpha, IL-6 and IL-8 release by human monocytes and alveolar macrophages. J Immunol 1993;150:2885-2891

23. Stephens RC, Fidler K, Wilson $\mathrm{P}$, et al. Endotoxin immunity and the development of the systemic inflammatory response syndrome in critically ill children. Intensive Care Med 2006;32:286-294

24. Strutz F, Heller G, Krasemann K, et al. Relationship of antibodies to endotoxin core to mortality in medical patients with sepsis syndrome. Intensive Care Med 1999;25:435444

25. Poeze M, Solberg BC, Greve JW, Ramsay G. Gastric PgCO2 and Pg-aCO2 gap are related to D-lactate and not to L-lactate levels in patients with septic shock. Intensive Care Med 2003;29:2081-2085

26. Gollin G, Marks C, Marks WH. Intestinal fatty acid binding protein in serum and urine reflects early ischemic injury to the small bowel. Surgery 1993;113:545-551

27. Kanda $\mathrm{T}$, Nakatomi $\mathrm{Y}$, Ishikawa $\mathrm{H}$, et al. Intestinal fatty acid-binding protein as a sensitive marker of intestinal ischemia. Dig Dis Sci 1992;37:1362-1367 


\section{Chapter 4}

28. Cerny V, Cvachovec K. Gastric tonometry and intramucosal pH--theoretical principles and clinical application. Physiol Res 2000;49:289-297

29. Welch SB, Nadel S. Treatment of meningococcal infection. Arch Dis Child 2003;88:608-614

30. Britto J, Nadel S, Habibi P, Levin M. Gastrointestinal perforation complicating meningococcal disease. Pediatr Infect Dis J 1995;14:393-394

31. McCaffrey DD, Lucas J, Gardiner KR. Haemorrhage from small bowel ulceration complicating meningococcal septicaemia. Ulster Med / 2005;74:57-59 


\subsection{Visceral injury and systemic inflammation in patients undergoing extracorporeal circulation during aortic surgery}

Sebastiaan J. Hanssen, Joep P. M. Derikx, Iris C. Vermeulen Windsant, John H. Heijmans, Thomas A. Koeppel, Geert Willem Schurink, Wim A. Buurman, Michael J. Jacobs

Annals of Surgery (2008) 248: 117-125. 


\begin{abstract}

\section{Objectives}

Visceral injury and inflammation are evaluated in patients undergoing extracorporeal circulation (ECC) either with distal aortic perfusion (DAP) during thoracic aortic aneurysm (TAA) repair or DAP and selective organ perfusion (DAP \& SP) during thoracoabdominal aortic aneurysm (TAAA) repair.
\end{abstract}

\title{
Summary Background Data
}

Visceral hypoperfusion and subsequent visceral injury, mainly to the gut, have been implicated as central events in the development of systemic inflammatory response syndrome (SIRS) and organ dysfunction after major surgery. Patients undergoing DAP or DAP \& SP are exposed to artificial visceral perfusion, potentially leading to the development of intestinal injury and systemic inflammation.

\section{Methods}

To assess visceral injury arteriovenous differences of Fatty Acid Binding Proteins were measured for the gut (I-FABP and L-FABP) and left kidney (L-FABP) along with systemic plasma concentrations. Systemic ALT was used as liver injury marker. Plasma IL-6 and IL-8 denoted systemic inflammation.

\section{Results}

During ECC systemic I-FABP and L-FABP levels increased in both groups, representing intestinal injury. Significantly elevated levels of I-FABP $(P<0.001)$ and L-FABP $(P<0.001)$ were found in the DAP \& SP group, after ECC was stopped and normal circulation restored. Liver and renal tubular cell injury was not detected. Significant increases in systemic IL- 6 and IL-8 values were measured only in patients undergoing DAP \& SP. Additionally, the extent of intestinal injury correlated positively with systemic inflammation.

\section{Conclusion}

This study shows the development of intestinal mucosal injury during ECC with DAP or DAP \& SP, indicative of insufficient intestinal perfusion. Intestinal injury was associated with a systemic pro-inflammatory response. 


\section{INTRODUCTION}

Open surgical repair of thoracic aortic aneurysms (TAA) and thoracoabdominal aortic aneurysms (TAAA) are extensive, complex procedures that are associated with significant post-operative morbidity and mortality. Post-operative complications include respiratory failure, renal and liver dysfunction, spinal cord injury, and gastrointestinal complications. ${ }^{1-3}$

Experimental studies of major surgery and trauma suggest that intestinal injury and hypoperfusion of visceral organs lead to initiation of a systemic proinflammatory response. ${ }^{4,5}$ Therefore, visceral protection during aortic surgery is warranted. ${ }^{6,7}$

The extracorporeal circulation (ECC) technique has been implemented in open TAA repair to provide (retrograde) distal aortic perfusion (DAP) via the femoral artery. In case of TAAA repair involving the origin of visceral arteries, ECC with DAP is combined with selective organ perfusion catheters (DAP \& SP) to provide designated visceral arteries directly with blood. ${ }^{8,9}$ The rationale behind DAP and DAP \& SP is to reduce ischemic injury of visceral organs, in particular the highly susceptible intestinal mucosa. ${ }^{10}$

The advantages of visceral protection notwithstanding, ECC during cardiac surgery provides antegrade aortic perfusion of visceral organs and is still associated with loss of intestinal barrier function seen as endotoxin translocation, indicating intestinal mucosal injury. ${ }^{11-13}$ The development of intestinal organ injury during antegrade aortic perfusion is strongly associated with gastrointestinal complications and mortality. ${ }^{14,} 15$ Moreover, recent publications suggest ECC with antegrade aortic flow to result in a poorly understood state of 'functional' hypoperfusion of visceral organs, which is related to visceral injury and the induction of SIRS. ${ }^{14,16,17}$

The quantification of (subclinical) visceral injury during open TAA / TAAA repair could provide valuable information on the perfusion adequacy of ECC with either DAP or DAP \& SP. Therefore, in this study we measured intestinal and renal release of fatty acid binding proteins, small cytosolic proteins that leak from injured cells into the circulation, and systemic plasma ALT to evaluate liver injury. The pro-inflammatory cytokines IL-6 and IL-8 were measured to assess systemic inflammation. The aim of the present study was to investigate the development of visceral injury in patients undergoing either DAP or DAP \& $\mathrm{SP}$, and to evaluate the relation of visceral injury to the systemic inflammatory response in these patients. 


\section{Methods}

\section{Patient Population}

Patients that underwent elective open TAA or TAAA repair between January 2006 and April 2007 at the University Hospital Maastricht or the University Hospital Aachen were included in the study. The study was approved by the local Institutional Review Board of both institutes, and written informed consent was obtained from every patient.

Pre-operative clinical data were prospectively collected from the patient's medical record. Crawford's classification was used to specify TAAA type. ${ }^{18}$

\section{Surgical Procedure}

In all patients the same anesthetic protocol was used. The following anesthetic and surgical procedure has been described previously in detail. ${ }^{9,}{ }^{19}$ Collapse of the left lung in all patients except type IV TAAA was enabled by either a doublelumen endotracheal tube or a selective left main bronchial blocker. Induction of anesthesia was achieved with etomidate and sufentanil, and maintained with sufentanil and ketamine. All patients were placed in a left helical position, and thoracotomy or thoracolaparotomy through the sixth intercostal space (eighth intercostal in patients with TAAA type IV aneurysms) was performed.

After heparinization, $(0.5 \mathrm{mg} / \mathrm{kg})$, for all patients ECC with DAP was established after cannulation of the left pulmonary vein and the femoral artery, connected to a centrifugal pump. Alternatively, the femoral vein was cannulated with a long cannula placed in the vena cava inferior close to the right atrium; in all cases an oxygenator was part of the ECC. For DAP \& SP, a 4-branched tubing system was connected to the ECC and 4 catheters with inflatable tips and flow probes were used for selective perfusion of the celiac axis, the superior mesenteric artery and both renal arteries.

Typically, the aortic reconstruction was done from proximal to distal. During proximal cross-clamping, transsection of the aorta, and completion of the proximal anastomosis, DAP was maintained at a mean pressure of 60 $\mathrm{mmHg}$ or higher. After abdominal aortotomy, all four selective perfusion catheters were installed in patients undergoing TAAA repair. In ten patients $(\mathrm{N}=10)$ we evaluated directly after insertion the mean blood flow through each individual SP catheter (see Table 1). 


\section{Gut wall integrity loss in critical illness and surgery}

Table 1: Characteristics of patients undergoing open TAA / TAAA repair.

\begin{tabular}{|c|c|c|c|}
\hline & $\begin{array}{l}\text { ECC with } \\
\text { DAP }\end{array}$ & $\begin{array}{l}\text { ECC with } \\
\text { DAP \& SP }\end{array}$ & $P$-value \\
\hline Total procedures (N) & 8 & 22 & \\
\hline TAA & 8 & - & \\
\hline TAAA * & - & 22 & \\
\hline TAAA // & - & 3 & \\
\hline TAAA III & - & 9 & \\
\hline TAAA IV & - & 10 & \\
\hline Male (N) & 4 & 11 & 1.000 \\
\hline Dissection & 4 & 2 & 0.033 \\
\hline Mean age (yr) & $60.0(3.4)$ & $63.5(2.7)$ & 0.483 \\
\hline $\mathrm{BMI}^{* *}\left(\mathrm{~kg} / \mathrm{m}^{2}\right)$ & $26.9(1.2)$ & $23.9(0.8)$ & 0.058 \\
\hline Diabetes & - & 2 & 1.000 \\
\hline Hypertension & 5 & 17 & 0.643 \\
\hline Smoked & 4 & 19 & 0.395 \\
\hline Hypercholesterolemia & 1 & 11 & 0.099 \\
\hline Chronic renal failure & - & 2 & 1.000 \\
\hline$C A D^{\wedge}$ & 1 & 2 & 1.000 \\
\hline $\mathrm{COPD}^{\wedge \wedge}$ & - & 5 & 0.280 \\
\hline ACC \# time (min) & $86(9)$ & $86(7)$ & 0.986 \\
\hline ECC time (min) & $131(36)$ & $142(9)$ & 0.677 \\
\hline \multicolumn{4}{|l|}{ Flow SP catheter ${ }^{\dagger}(\mathrm{mL} / \mathrm{min})$} \\
\hline Celiac axis & - & $202.0(33.2)$ & \\
\hline Superior mesenteric artery & - & $311.6(30.4)$ & \\
\hline Left renal artery & - & $212.6(33.3)$ & \\
\hline Right renal artery & - & $186.4(27.7)$ & \\
\hline Total operation time (min) & $326(17)$ & $399(15)$ & 0.015 \\
\hline
\end{tabular}

Classification according to Crawford..$^{18 *} \mathrm{BMI}$, body mass index; ${ }^{\wedge} \mathrm{CAD}$, coronary artery disease; ${ }^{\wedge}$ COPD, chronic obstructive pulmonary disease. ${ }^{\#}$ ACC, aorta cross clamp; Continues variables are expressed as mean (SEM). HFlow in SP catheter was measured in ten $(\mathrm{N}=10)$ patients undergoing ECC with DAP \& SP. 


\section{Chapter 4}

In general, the celiac axis, superior mesenteric artery and the right renal artery were anastomosed simultaneously as an island in the aortic graft. Just before finishing the anastomosis, the perfusion catheters were removed, indicating that ischemic time is limited to the timeframes of insertion and removal of the catheters. In most cases, the left renal artery was treated with a selective graft using selective perfusion during end-to-end anastomosis. After completion of the anastomosis, the tip of the catheter was withdrawn into the graft, and perfusion continued, until implantation into the aortic tube graft was accomplished.

\section{Blood Sampling}

Peripheral arterial blood was drawn at set time-points (Fig. 1) from the radial artery pre-operatively (Pre-op), while creating access to the aorta, but before clamping the aorta and ECC was initiated (Cannulation), during ECC just before ending DAP or DAP \& SP (ECC), 15 minutes after the proximal aortic cross clamp was removed and reperfusion (R) initiated ( $15 \mathrm{~min} R$ ), at 2 hours, 6 hours and 10 hours in the reperfusion phase ( 2 hrs R, 6 hrs $R$ and $10 \mathrm{hrs} R$ ), as well as at day 1 and 2 postoperatively (Day 1 and Day 2). Whole blood was collected in EDTA vacutainers (Becton Dickinson, Franklin Lakes, NJ) and immediately put on ice. Blood was centrifuged and plasma was stored in aliquots at $-20^{\circ} \mathrm{C}$ until further analysis.

To enable venous blood sampling from the intestines and left kidney in eight patients $(\mathrm{N}=8)$ undergoing DAP \& SP, a $16 \mathrm{G}$ single lumen central venous catheter kit was used to cannulate the portal vein (Terumo Corporation, Tokyo, Japan), and a $18 \mathrm{G}$ intravenous catheter (Venflon, Becton Dickinson, Franklin Lakes, NJ) was used to cannulate the left renal vein prior to starting ECC. This enabled us to sample specific arterial and venous blood from the gut and the left kidney in order to calculate organ release and consumption of plasma proteins. Blood was taken from these cannulas before ECC, during ECC with DAP \& SP when a sample was taken from the arterial line of the ECC as well, and at 15 minutes reperfusion (Fig. 1). 
Gut wall integrity loss in critical illness and surgery

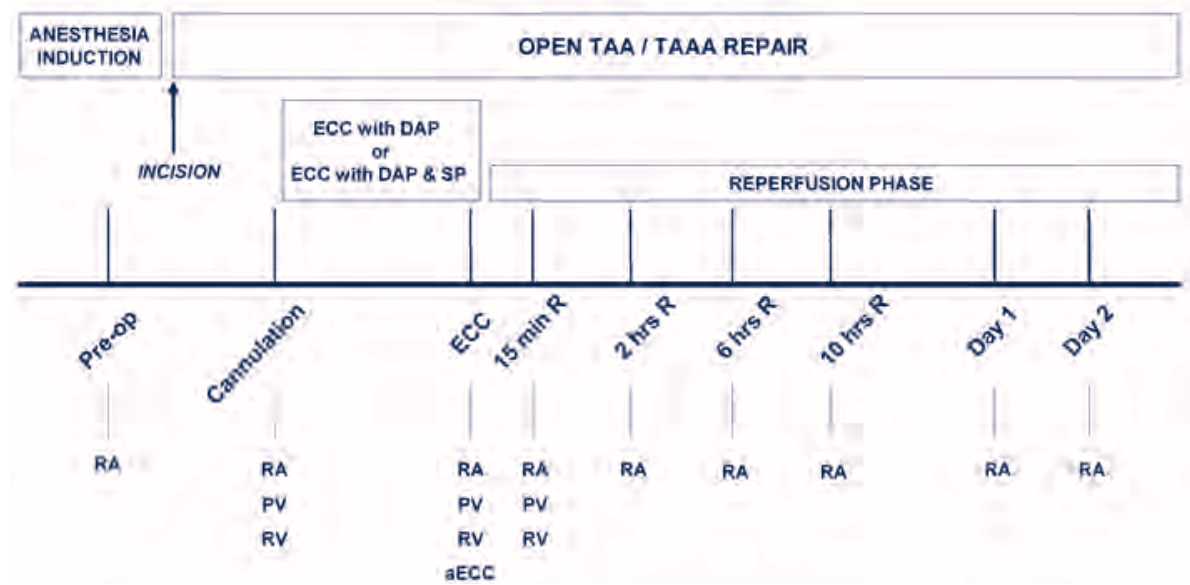

Figure 1: Timeline of blood sampling. At given time-points blood was drawn from the radial artery line and arterial line of the extracorporeal circulation (ECC). Patients undergoing open TAA repair received ECC with distal aortic perfusion (DAP-group), whereas ECC with DAP and selective organ perfusion was used in patients undergoing open TAAA repair (DAP and SP-group). To enable simultaneaous arterial and venous organ blood sampling, in 8 patients receiving ECC with DAP and SP, a venous catheder was inserted in the portal vein, as well as the left renal vein. This allowed specific calculation of arteriovenous concentration differences for the gut as well as the left kidney. PV, portal vein; RV, renal vein; aECC, arterial line of ECC.

\section{Measurements}

\section{Visceral Organ Injury}

Fatty acid binding proteins (FABP) are small cytosolic proteins (15 kDa) that are abundantly expressed in visceral tissue and can be used as sensitive plasma markers to detect early cellular injury. ${ }^{20,22}$ Two types of FABP, intestinal-type FABP (I-FABP) and liver-type (L-FABP), are expressed at the tips of the villi of intestinal mucosal epithelial cells. I-FABP is only present in intestinal mucosal cells; in contrast, L-FABP is also expressed in hepatocytes, and to a lesser extent in renal tubular cells, and increased plasma levels could therefore represent hepatocyte or renal tubular cell injury as well. ${ }^{23}$ In order to evaluate hepatocellular injury systemic common liver injury marker ALT was measured. Furthermore, to study renal tubular cell injury as cause of elevated circulating L-FABP levels, the arteriovenous concentration difference for the left kidney ([arterial concentration] - [venous concentration] / [arterial concentration] $\mathrm{x}$ $100 \%)$ of L-FABP was calculated in eight patients $(\mathrm{N}=8)$ undergoing DAP \& SP.

Plasma I-FABP and L-FABP levels were analyzed using Enzyme Linked Immuno Sorbent Assays (ELISA). These commercially available ELISA kits were 


\section{Chapter 4}

kindly provided by Hycult biotechnology (Uden, the Netherlands) and were used according to manufacture guidelines. The detection limit was $41 \mathrm{pg} / \mathrm{mL}$ for I-FABP and $102 \mathrm{pg} / \mathrm{mL}$ for L-FABP.

\section{Systemic Inflammatory Response}

The plasma levels of the cytokines IL- 6 and IL- 8 were assayed to determine the systemic inflammatory response following TAA / TAAA repair. IL-6 and IL-8 are commonly used as general inflammatory markers, amongst others produced by stimulated monocytes and activated endothelium, and considered key players in the development of SIRS after major trauma and surgical stress. ${ }^{17,24,25}$

IL-6 and IL-8 ELISA techniques have been described elsewhere. ${ }^{26}$ Briefly, 96-well plates (Greiner Bio-One, Kremsmünster, Austria) were coated overnight $\left(4^{\circ} \mathrm{C}\right)$ with the appropriate antibodies. Monoclonal antibodies 5E1 and 3E3 were used as coating for IL-6 and IL-8, respectively. Free sites were blocked with $1 \%$ bovine serum albumin in PBS.

Samples and standard dilution series were incubated for 2 hours. Human recombinant IL- 6 and IL-8 were used for standard titration curves. Washing buffer contained $0.1 \%$ Tween-20. Biotinylated anti-human IL-6 and IL-8 were developed in our own laboratory and used as detection antibodies. Finally, 3,3',5, $5^{\prime}$-tetramethyl-benzidine (TMB) was used as a substrate and the reaction was stopped by adding $1 \mathrm{M} \mathrm{H} 2 \mathrm{SO} 4$. Plasma samples were analyzed in the same run spectrophotometrically $(450 \mathrm{~nm})$ using an automated ELISA reader. The detection limits for the assays were $12.3 \mathrm{pg} / \mathrm{mL}$ (IL-6) and $46.0 \mathrm{pg} / \mathrm{mL}$ (IL-8).

\section{Statistical Analysis}

All data are expressed as mean \pm standard error of the mean (SEM). KolmogorovSmirnov test was used to test for Gaussian distribution. Student's T-test for independent-samples and Chi-square test, followed by Fisher's exact test when needed, were used to test differences between pre-operative patient characteristics. Changes in parameters over time were tested using repeated measures ANOVA, followed by paired Student's T-tests versus Pre-op values with post-hoc Bonferroni correction, if significant differences were found. In the same way changes in arteriovenous concentrations differences of plasma proteins were tested, with values measured during Cannulation as reference. To characterize the total amount of intestinal injury and systemic inflammation during the study period, area under the curve (AUC) for I-FABP and L-FABP, as well as IL-6 and IL8 was calculated for each patient. To assess association between intestinal mucosal cell injury and inflammation Spearman correlation analysis between $A \cup C_{\text {I-FABP }}$ $A \cup C_{\mathrm{L}-\mathrm{FABP}}, \mathrm{AUC}_{\mathrm{IL}-\mathrm{G}^{\prime}}$ and $\mathrm{AUC}_{\mathrm{IL}-8}$ was performed. Mann Whitney $U$ test was used 
to calculate differences in AUC for each parameter between both patient groups. Statistical calculations were made using SPSS 15.0 for Windows (SPSS, Inc., Chicago, IL), and GraphPad Prism software (GraphPad Software, Inc. San Diego, CA). A P-value $<0.05$ was considered to indicate statistical significance.

\section{ResUlts}

\section{Patient Characteristics and Intra-Operative Data}

Thirty consecutive patients that underwent TAA $(\mathrm{N}=8)$ or TAAA $(\mathrm{N}=22)$ repair were included in the study. Table 1 shows pre-operative clinical data of patients undergoing ECC with DAP (TAA patients) or DAP \& SP (TAAA patients). No significant differences in pre-existing co-morbidities were present in the two patient groups studied, except for the co-existence of an aortic dissection (DAP $\mathrm{N}=4 / 8$ versus DAP \& SP $\mathrm{N}=2 / 22, \mathrm{P}=0.033$ ). Nevertheless, aortic dissection did not influence the surgical approach to repair the aneurysm.

Aorta cross-clamp time (ACC), total ECC time and total operation time were noted during all procedures. ACC and total ECC time did not differ significantly between both groups. However, a significant difference in total operation time was found, reflecting the operative complexity of patients undergoing TAAA repair (DAP 326 (17) minutes, and DAP \& SP 399 (15) minutes, $\mathrm{P}=0.015$ ).

\section{Extracorporeal Circulation and Intestinal Mucosal Injury}

Pre-operative I-FABP levels (DAP 326.8 (84.8) pg/mL, and DAP \& SP 411.7 (82.4) $\mathrm{pg} / \mathrm{mL}$, Fig. $2 A$ ) were comparable to the values measured in a healthy population, ${ }^{27}$ demonstrating the absence of intestinal mucosal cell injury. Manipulation of visceral organs to create access to the aorta, did not lead to elevated circulating I-FABP levels. In contrast, during ECC I-FABP levels increased in both groups representing the development of intestinal mucosal injury which was supported by statistically significant repeated measures ANOVA in both groups (DAP, $\mathrm{P}<0.05$; DAP \& SP, P $<0.0001$, data not shown). Shortly after artificial circulation was terminated and normal circulation was restored, plasma I-FABP levels increased further (DAP 763.9 (200.7) pg/mL, P > 0.05; DAP \& SP $2351.0(378.1) \mathrm{pg} / \mathrm{mL}, \mathrm{P}<0.001)$ and remained elevated for six hours. The release of I-FABP in patients undergoing DAP \& SP was clearly more pronounced than in patients undergoing DAP, indicating more intestinal injury in the DAP \& SP group. Total AUC of systemically measured I-FABP was calculated for both groups and revealed significantly more I-FABP release in the DAP \& SP patient group $(\mathrm{P}<0.05$, data not shown). 


\section{Chapter 4}
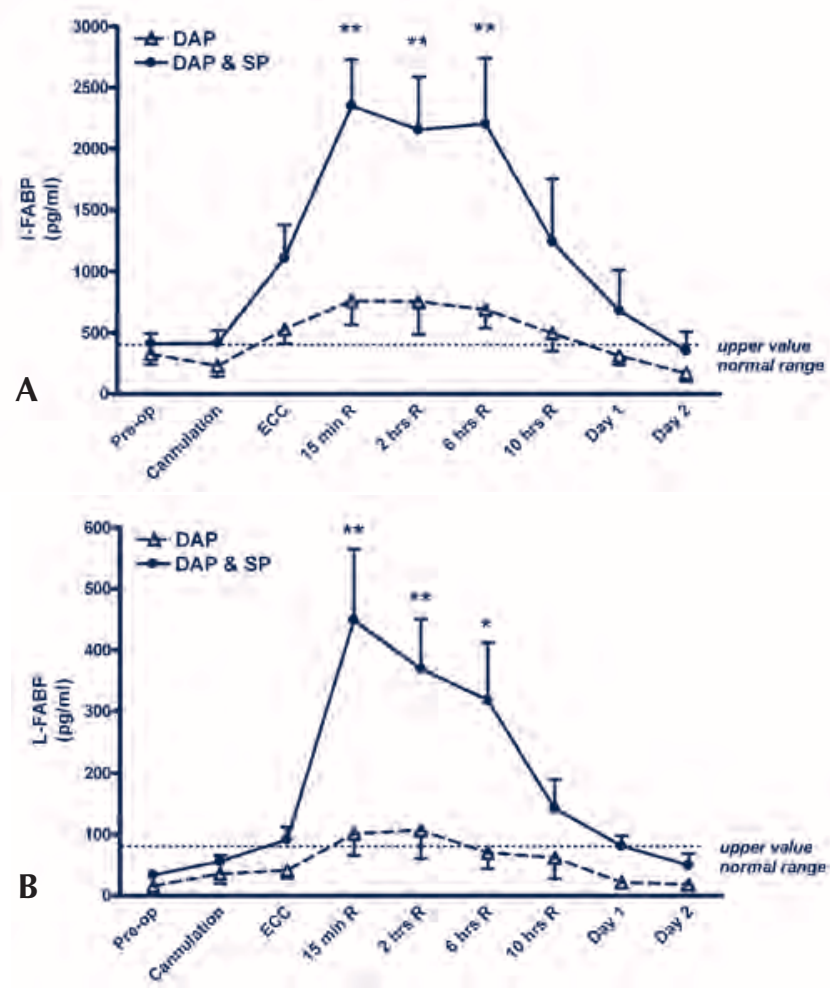

Figure 2: Elevated plasma I-FABP and L-FABP levels in patients undergoing DAP or DAP and SP. $A$, highest values for I-FABP reflecting intestinal injury were found during reperfusion for both groups. B, systemic plasma L-FABP values measured in both patient groups mimicked I-FABP release, potentially represented as mean \pm SEM and are compared with preoperative values. Data were analyzed using paired Student $t$-test with posthoc Bonferroni correction. ${ }^{*} \mathrm{P}<0.01 ;{ }^{* *} \mathrm{P}<$ 0.001

Intestinal mucosal cell injury leads next to I-FABP release to L-FABP release, although, plasma $\mathrm{L}-\mathrm{FABP}$ increase can also be caused by liver and renal tubular cell injury. L-FABP plasma levels showed a similar pattern as IFABP, potentially supporting intestinal injury (Fig. 2B). The total calculated $A \cup C_{L-F A B P}$ was significantly higher in patients undergoing DAP \& SP $(P<0.01$, data not shown).

To ascertain the intestinal origin of the released I-FABP and L-FABP during and shortly after ECC, the arteriovenous concentration differences were measured in eight patients $(N=8)$ undergoing DAP \& SP (Fig. 3A). In line with 
the systemic measurements, before starting ECC there was no fractional increase in I-FABP indicating the absence of I-FABP release (+ $0.3(1.8) \%$ ). However, during ECC with DAP \& SP a significant release of I-FABP was found (+ $53.5(11.2) \%, \mathrm{P}<0.001)$, which still occurred at 15 minutes after ECC had been stopped $(+28.0(3.3) \%, \mathrm{P}<0.05)$. Similar to the I-FABP release reported above, no detectable L-FABP release from the gut was found before starting ECC with DAP \& SP (+ 1.9 (2.8) \%). During ECC with DAP \& SP, L-FABP release from the intestinal mucosa increased significantly $(+103.0(17.5) \%, P<0.001)$ and release of L-FABP, specifically indicating intestinal injury, was still statistically significant shortly after ECC perfusion was terminated and normal circulation was restored $(+51.5(13.9) \%, \mathrm{P}<0.05)$.

Since L-FABP is next to the gut also expressed in liver and kidneys, we studied hepatocellular and renal tubular cell injury. To exclude liver injury, ALT was measured at three different time-points (Fig. 3B); ALT, a cytosolic enzyme mainly expressed in the liver, was not elevated in the early reperfusion phase or at post-operative day 1 . These data support the intestinal origin of L-FABP release during open TAA / TAAA repair.

Potentially, renal tubular cell injury also results in elevated plasma L-FABP concentrations. Therefore, to evaluate release from injured renal tubular cells, we measured arteriovenous concentration differences of L-FABP for the left kidney from the same eight patients as reported above. The calculated mean arteriovenous concentration difference before going on ECC was - 21.5 (4.0) \%. During ECC with DAP \& SP (- 27.6 (6.0) \%) and after ECC, when the patients were on normal visceral circulation again (- $22.8(4.4) \%)$, arteriovenous concentration differences were not significantly altered compared to values before ECC, indicating an absence of release from injured renal tubular cells (data not shown).

To analyze correlations between intestinal organ injury markers I-FABP and L-FABP, the total $A \cup C_{\text {I-FABP }}$ and $A \cup C_{\mathrm{L}-F A B P}$ of all patients undergoing ECC $(\mathrm{N}=30)$ were correlated (Fig. 5). A strong positive correlation was found between $A \cup C_{\text {I-FABP }}$ and $A \cup C_{\text {L-FABP }}(r 0.70, P<0.0001)$.

Taken together, the circulating plasma levels of both I-FABP and L-FABP were elevated during ECC and a further increase was observed in the early phase of restored normal circulation in patients undergoing open TAA / TAAA repair. This indicates intestinal injury, with release of these proteins being more prominent when ECC with DAP \& SP was used. 


\section{Chapter 4}
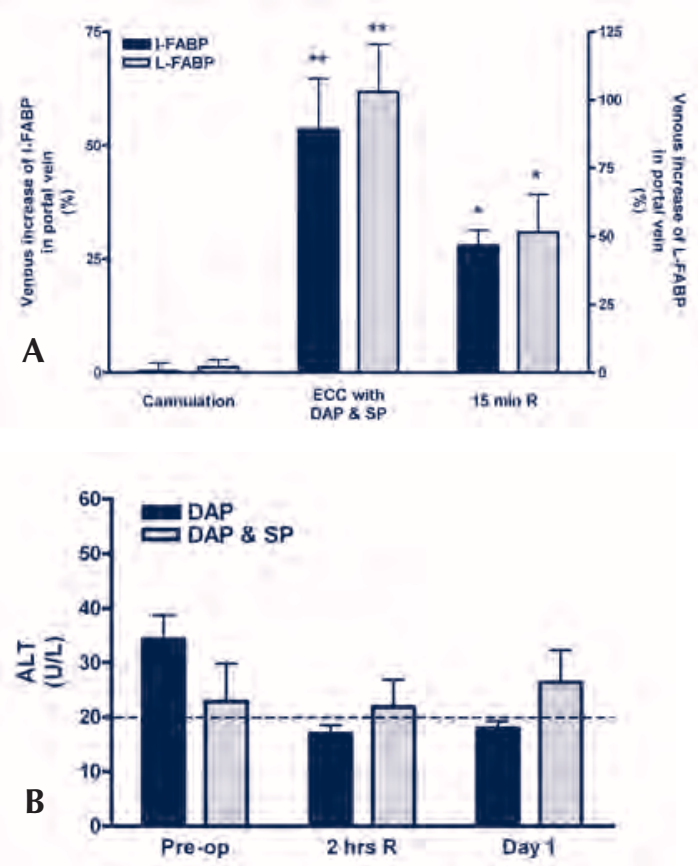

Figure 3: Intestinal I- FABP and intestinal L-FABP release was evaluated using arteriovenous concentration difference in 8 patients undergoing ECC with DAP and SP. Arterial blood from the radial artery (or the arterial line of the ECC) and the portal vein were simultaneously sampled and intestinal I-FABP and L-FABP release was calculated using the following formula: venous concentration minus arterial concentration divided by arterial concentration $\times 100 \% \mathrm{~A}$, significant release of I-FABP and L-FABP from the gut was observed during ECC and directly in the reperfusion phase when normal visceral circulation was restored. B, circulating concentrations ALT, a specific liver enzyme was measured preoperatively, at 2 hours reperfusion and at day 1 postoperatively. ALT levels showed no signifficant increase at the given time points, indicating no significant liver injury. Values are shown as mean \pm SEM and compared with preoperative values (or values found during Cannulation for Fig. 3A) using paired Student $t$-tests with posthoc Bonferroni correction. ${ }^{*} \mathrm{P}<0.05 ;{ }^{* *} \mathrm{P}<0.001$

Systemic Inflammatory Response and Extracorporeal Circulation during TAA / TAAA repair

Circulating pro-inflammatory cytokines IL-6 and IL-8 were measured in order to evaluate the inflammatory response associated with TAA / TAAA repair. Plasma concentrations of IL- 6 before ECC started were not significantly different from 
baseline values (Fig. 4A). Although changes in plasma IL-6 levels were observed in both patient groups, the mean IL-6 values measured in the DAP patients group were not statistically significantly altered during the studied period (ANOVA P $>0.05$, data not shown). During visceral perfusion using ECC combined with DAP \& SP plasma IL-6 levels increased significantly, indicating the development of a systemic inflammatory response (ANOVA $\mathrm{P}<0.0001$, data not shown). Release of IL-6 continued until peak-values were reached 2 hours after reperfusion (DAP \& SP 295.7 (62.7) pg/mL, P < 0.001), whereas after 6 hours reperfusion and later plasma values lowered, but remained elevated until day 2 post-operative.

The pro-inflammatory cytokine IL-8, a strong neutrophil attractant, was detectable in pre-operatively taken blood samples from five out of twenty-two patients who underwent DAP \& SP (Fig. 4B). Initially, plasma IL-8 curves mimicked IL-6 kinetics, starting to increase during the reperfusion phase in both groups. However, further increase in plasma IL-8 levels in patients who underwent ECC with DAP was relatively small (ANOVA P $>0.05$, data not shown), whereas increase in plasma IL-8 was significant in patients who underwent ECC with DAP \& SP (ANOVA P $<0.0001$, data not shown), with peak values found at 2 hours reperfusion (227.4 (43.5) pg/mL, P $<0.05$ ), indicating a strong pro-inflammatory, chemotactic response in these patients. At postoperative day 2, plasma IL-8 had almost disappeared, being non-detectable in patients who underwent DAP, and only detectable in plasma from four out of twenty-two patients who underwent DAP \& SP.

The increase of IL- 6 and IL-8 observed during TAA / TAAA repair, indicates the development of a systemic inflammatory response after DAP or DAP \& SP; IL-8 release was significant in patients undergoing DAP \& SP only, signifying a strong systemic neutrophil attractive response in this patient group. 


\section{Chapter 4}

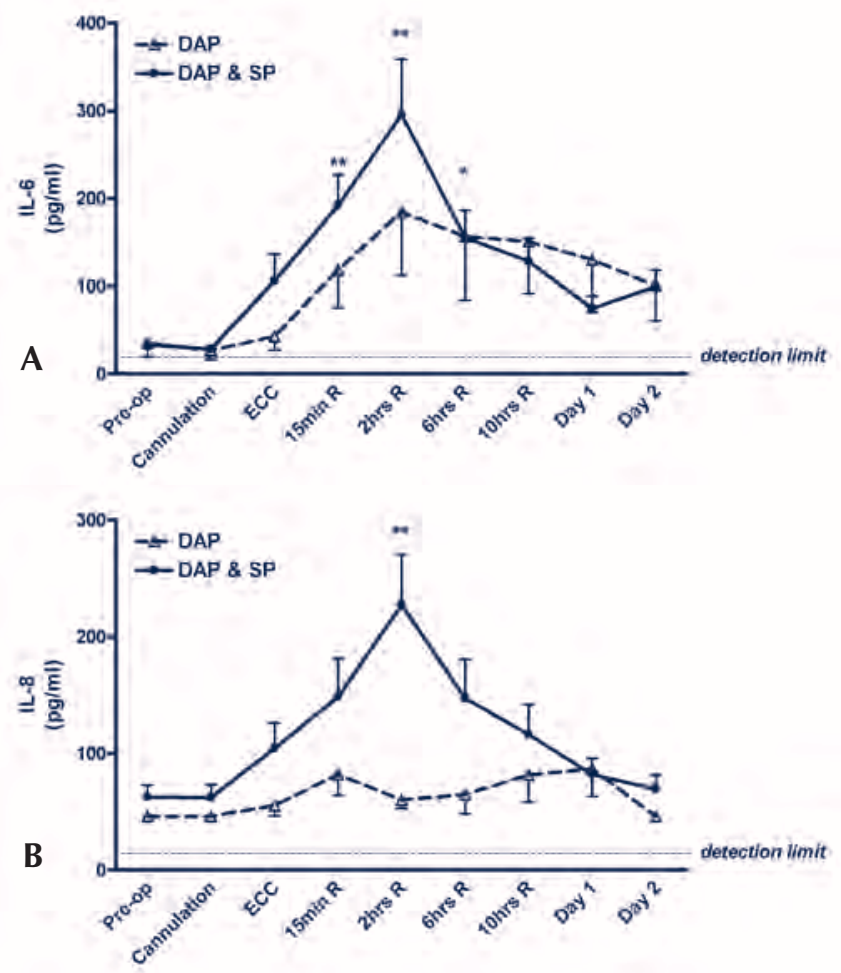

Figure 4: The systemic inflammatory response is initiated during ECC.A, increased levels of the pro-inflammatory cytokine IL-6 are found in patients receiving DAP and SP (TAAA patients) during artificial visceral perfusion using ECC. Peak values were reached at 2 hours reperfusion (2 hours R). B, marked IL-8 release in patients receiving DAP and SP. After initial release during ECC combined with DAP and SP, peak plasma values for patients undergoing TAAA repair were found at 2 hours reperfusion (2 hours R). Values are mean \pm SEM and compared with preoperative values using paired Student $t$-tests with posthoc Bonferroni correction. ${ }^{*} \mathrm{P}<0.05 ;{ }^{* *} \mathrm{P}<0.001$.

\section{Intestinal Injury and Systemic Inflammation in Patients Undergoing Extracorporeal Circulation}

To investigate whether intestinal injury was related to the systemic inflammatory response, correlations of $\mathrm{AUC}_{\mathrm{I}-\mathrm{FABP}}$ with $\mathrm{AUC}_{\mathrm{IL}-6}$ and $\mathrm{AUC}_{\mathrm{IL}-8}$ (Fig. 5) were analyzed in all patients undergoing ECC $(\mathrm{N}=30)$. $A \cup C_{\text {I-FABP }}$ correlated significantly with both $\mathrm{AUC}_{\mathrm{IL}-6}(\mathrm{r} 0.64, \mathrm{P}<0.001)$ and $\mathrm{AUC}_{\mathrm{IL}-8}(\mathrm{r} 0.54, \mathrm{P}<0.01)$, indicating an association between the extent of intestinal organ injury and systemic inflammation, underlining the need for intestinal organ protection during TAA / TAAA repair. 


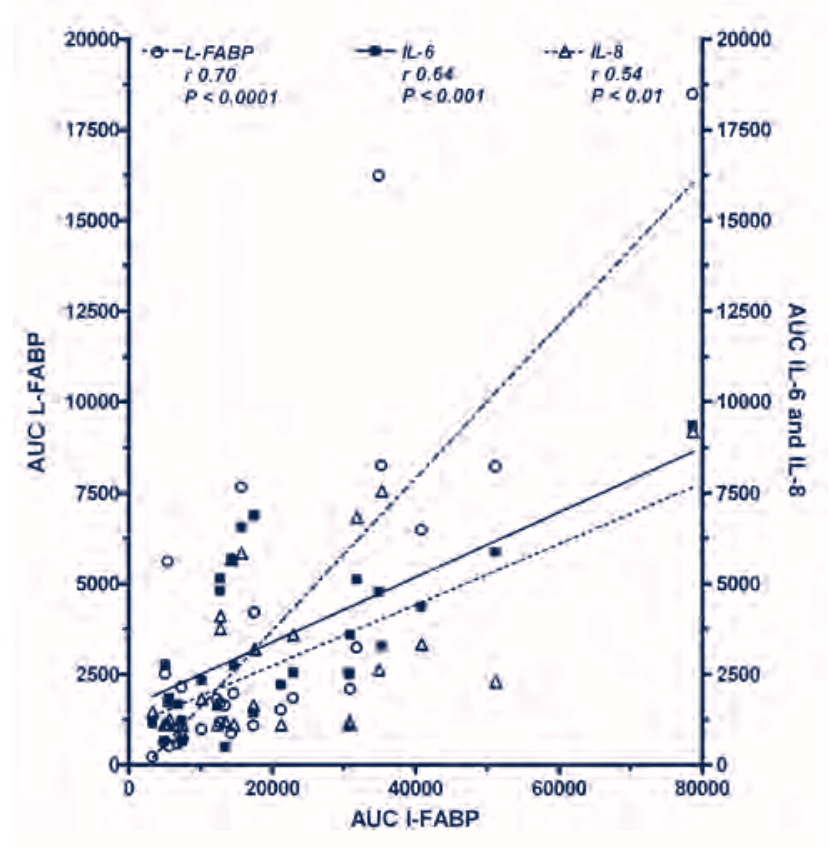

Figure 5: Association between intestinal mucosal cell injury and systemic inflammation in patients undergoing open TAA/TAAA repair. Total AUCs of I-FABP, L-FABP, IL-6, and IL-8 of the patient were measured from preoperative up until day 2 postoperative. Data are matched observations, and degree of correlation is represented by Spearman r. Visceral injury, denoted by

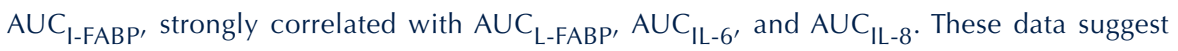
intestinal injury to play a significant role in systemic inflammation in patients undergoing open TAA/TAAA repair.

\section{Discussion}

In this study intestinal mucosal cell injury, but not hepatic or renal tubular cell injury, occurred in patients undergoing elective open TAA or TAAA repair, despite artificial visceral perfusion using ECC with either DAP (TAA patients) or ECC with DAP \& SP (TAAA patients). The extent of intestinal injury and proinflammatory reaction was more pronounced in patients undergoing DAP \& SP. Furthermore, intestinal injury correlated positively with markers of systemic inflammation.

Visceral perfusion by means of ECC is used to ensure volume and pressure controlled blood flow to abdominal organs, preventing the deleterious effects of 


\section{Chapter 4}

intestinal injury. ${ }^{3}$ ECC with antegrade aortic perfusion is a well-known technique used in cardiac surgery and acknowledged to induce intestinal injury. ${ }^{11}$ Holmes et al. showed elevated urine I-FABP levels following CABG surgery and suggested that intestinal mucosal injury plays a role in inducing a complicated post-operative course. ${ }^{14}$ The use of ECC with either DAP or DAP \& $\mathrm{SP}$ in patients undergoing open TAA / TAAA repair was first described in the 1980s, and these techniques have since been further refined. ${ }^{28,}{ }^{29}$ We described our first experiences with ECC and DAP or DAP \& SP in the mid-90s, and both were considered safe techniques to prevent postoperative renal injury, assessed by urine output and serum creatinine following TAA / TAAA repair, and possibly intestinal injury, by subjective observation of the intestines and intestinal motility peri- and post-operatively. ${ }^{8,} 19$

In the present study, the development of injury to visceral organs was investigated using measurements of plasma I-FABP and L-FABP for intestinal, ALT for hepatic, and L-FABP for renal tubular injury. Our data show development of intestinal injury during artificial perfusion of the viscera using ECC, and shortly thereafter when normal perfusion to the viscera was restored. ECC with DAP caused elevated plasma levels of I-FABP and L-FABP, and ECC with DAP \& SP led to significantly elevated circulating I-FABP and L-FABP levels, despite the intended protective effect of artificial visceral perfusion in patients undergoing open TAA / TAAA repair. The arteriovenous concentration differences over the intestines demonstrate significant release of I-FABP and LFABP in patients undergoing ECC with DAP \& SP. During reperfusion the systemic plasma concentration of both I-FABP and L-FABP initially further increased, suggesting either continued wash-out of these proteins from the injured gut mucosa or sustained hypoperfusion injury leading to continued enhanced release of both proteins. These data are in line with low flow states in patients with hypovolemic or cardiogenic shock and inherent blood flow redistribution towards the heart and central nervous system at the expense of visceral organs. The intestinal mucosal epithelial cells are due to the specific anatomy of the small intestinal villus more susceptible to visceral hypoperfusion than hepatocytes or renal epithelial cells. In particular the cells at the tip of the villus, which contain I-FABP and L-FABP, are due to the sympathetic innervation of the arterioles and vascular anatomy of the villus, most vulnerable to hypoperfusion. ${ }^{10,11}$ At this moment we investigate the mechanisms that potentially underlie this visceral hypoperfusion.

Total intestinal injury seen during ECC was assessed by $\mathrm{AUC}_{\mathrm{I-FABP}}$, and correlated positively with the $\mathrm{AUC}_{\mathrm{IL}-6}$ and $\mathrm{AUC}_{\mathrm{IL}-8}$, suggesting an association between the extent of intestinal mucosal injury and the extent of systemic 
inflammation. Morariu et al. (2005) studied the alleged protective use of dexamethasone on the inflammatory response and intestinal injury after CABG surgery with the use of antegrade aortic perfusion. ${ }^{17}$ Even though the inflammatory response was attenuated by dexamethasone, no effect on intestinal injury markers was seen, hereby suggesting the inflammatory response to be a result and not so much a cause of intestinal injury.

Pharmacological approaches to reduce visceral hypoperfusion and its sequelae can be directed at either improvement of perfusion or reduction of ischemic injury. Increasing microcirculatory blood flow, especially in the intestines, by pharmacological intervention (e.g. ACE-inhibitors, ET-receptor antagonists or catecholamine therapy) has been shown beneficial in animal studies, ${ }^{30,} 32$ Furthermore, also antioxidant therapy (e.g. nitroxide, ascorbic acid or mannitol) has been shown effective in reducing ischemic injury to the intestines in animals. ${ }^{33,} 34$ Future studies are needed to evaluate such interventions in the human setting.

The differences in extent of intestinal injury that was observed between DAP during open TAA repair and DAP \& SP during open TAAA repair is not easily clarified. Aortic cross clamp times or total perfusion times were similar between the groups. The most likely explanation for the difference in intestinal injury is a more prominent hypoperfusion of the intestines during ECC with DAP \& SP. Idu et al. showed in a pig model a significant decrease in blood flow per minute to the cranial mesenteric artery when clamping the aorta and perfusion was taken over with ECC and selective organ perfusion. ${ }^{35}$ Also, Leijdekkers et al. calculated that the flow to visceral organs during ECC with DAP \& SP is limited and insufficient to maintain tissue perfusion due to the internal diameter in SP catheters used in patients undergoing open TAAA repair. ${ }^{36}$ The flow administered through the selective organ perfusion catheter system during aortic clamping may be too low in our patients, creating an absolute state of 'functional' hypoperfusion to the intestines. It is of interest however that during the reperfusion phase especially in the DAP \& SP group the intestinal injury further increases. This suggests that during ECC a process has been initiated that results in ongoing increased intestinal mucosal cell injury, potentially through a prolonged 'functional' hypoperfusion. The mechanisms leading to visceral hypoperfusion during and after ECC in patients undergoing open TAA / TAAA repair remain to be resolved. Such information will be instrumental in developing strategies to prevent the intestinal injury and subsequent systemic inflammatory response induced by ECC with DAP or DAP \& SP. 


\section{Chapter 4}

\section{Conclusion}

Increased plasma I-FABP and L-FABP levels, representative of intestinal injury, and elevated plasma IL-6 and IL-8 concentrations, indicative of systemic inflammation, were observed in patients undergoing open TAA / TAAA repair, despite ECC with either DAP or DAP \& SP. Our data show that intestinal injury precedes the development of a systemic inflammatory response and correlates positively with the extent of systemic inflammation, supporting the hypothesis that intestinal tissue injury is involved in the development and extent of a systemic inflammatory response.

\section{ReferenCes}

1. Rectenwald JE, Huber TS, Martin TD, et al. Functional outcome after thoracoabdominal aortic aneurysm repair. J Vasc Surg 2002; 35(4):640-7.

2. Gloviczki P. Surgical repair of thoracoabdominal aneurysms: patient selection, techniques and results. Cardiovasc Surg 2002; 10(4):434-41.

3. Achouh PE, Madsen K, Miller CC, 3rd, et al. Gastrointestinal complications after descending thoracic and thoracoabdominal aortic repairs: a 14-year experience. J Vasc Surg 2006; 44(3):442-6.

4. Fink MP. Gastrointestinal mucosal injury in experimental models of shock, trauma, and sepsis. Crit Care Med 1991; 19(5):627-41.

5. Moore FA. The role of the gastrointestinal tract in postinjury multiple organ failure. Am J Surg 1999; 178(6):449-53.

6. MacArthur RG, Carter SA, Coselli JS, LeMaire SA. Organ protection during thoracoabdominal aortic surgery: rationale for a multimodality approach. Semin Cardiothorac Vasc Anesth 2005; 9(2):143-9.

7. Welborn MB, Oldenburg HS, Hess PJ, et al. The relationship between visceral ischemia, proinflammatory cytokines, and organ injury in patients undergoing thoracoabdominal aortic aneurysm repair. Crit Care Med 2000; 28(9):3191-7.

8. Jacobs MJ, de Mol BA, Legemate DA, et al. Retrograde aortic and selective organ perfusion during thoracoabdominal aortic aneurysm repair. Eur I Vasc Endovasc Surg 1997; 14(5):360-6.

9. Jacobs MJ, Mommertz G, Koeppel TA, et al. Surgical repair of thoracoabdominal aortic aneurysms. J Cardiovasc Surg (Torino) 2007; 48(1):49-58.

10. Takala J. Determinants of splanchnic blood flow. Br J Anaesth 1996; 77(1):50-8. 
11. Ohri SK, Somasundaram S, Koak Y, et al. The effect of intestinal hypoperfusion on intestinal absorption and permeability during cardiopulmonary bypass. Gastroenterology 1994; 106(2):318-23.

12. Riddington DW, Venkatesh B, Boivin CM, et al. Intestinal permeability, gastric intramucosal $\mathrm{pH}$, and systemic endotoxemia in patients undergoing cardiopulmonary bypass. Jama 1996; 275(13):1007-12.

13. Rossi M, Sganga G, Mazzone M, et al. Cardiopulmonary bypass in man: role of the intestine in a self-limiting inflammatory response with demonstrable bacterial translocation. Ann Thorac Surg 2004; 77(2):612-8.

14. Holmes JHt, Lieberman JM, Probert CB, et al. Elevated intestinal fatty acid binding protein and gastrointestinal complications following cardiopulmonary bypass: a preliminary analysis. J Surg Res 2001; 100(2):192-6.

15. Bolcal C, lyem H, Sargin M, et al. Gastrointestinal complications after cardiopulmonary bypass: Sixteen years of experience. Can J Gastroenterol 2005; 19(10):613-7.

16. Sato K, Sogawa M, Namura O, Hayashi J. Deterioration of body oxygen metabolism by vasodilator and/or vasoconstrictor administration during cardiopulmonary bypass. Asaio / 2006; 52(1):96-9.

17. Morariu AM, Loef BG, Aarts LP, et al. Dexamethasone: benefit and prejudice for patients undergoing on-pump coronary artery bypass grafting: a study on myocardial, pulmonary, renal, intestinal, and hepatic injury. Chest 2005; 128(4):2677-87.

18. Crawford ES. Thoraco-abdominal and abdominal aortic aneurysms involving renal, superior mesenteric, celiac arteries. Ann Surg 1974; 179(5):763-72.

19. Jacobs MJ, Eijsman L, Meylaerts SA, et al. Reduced renal failure following thoracoabdominal aortic aneurysm repair by selective perfusion. Eur / Cardiothorac Surg 1998; 14(2):201-5.

20. Lieberman JM, Sacchettini J, Marks C, Marks WH. Human intestinal fatty acid binding protein: report of an assay with studies in normal volunteers and intestinal ischemia. Surgery 1997; 121(3):335-42.

21. Niewold TA, Meinen M, van der Meulen J. Plasma intestinal fatty acid binding protein (I-FABP) concentrations increase following intestinal ischemia in pigs. Res Vet Sci 2004; 77(1):89-91.

22. Pelsers MM, Namiot Z, Kisielewski W, et al. Intestinal-type and liver-type fatty acidbinding protein in the intestine. Tissue distribution and clinical utility. Clin Biochem 2003; 36(7):529-35.

23. Pelsers MM, Hermens WT, Glatz JF. Fatty acid-binding proteins as plasma markers of tissue injury. Clin Chim Acta 2005; 352(1-2):15-35.

24. Ytting $\mathrm{H}$, Christensen IJ, Basse L, et al. Influence of major surgery on the mannanbinding lectin pathway of innate immunity. Clin Exp Immunol 2006; 144(2):239-46. 


\section{Chapter 4}

25. Fiane AE, Videm V, Lingaas PS, et al. Mechanism of complement activation and its role in the inflammatory response after thoracoabdominal aortic aneurysm repair. Circulation 2003; 108(7):849-56.

26. Dentener MA, Bazil V, Von Asmuth EJ, et al. Involvement of CD14 in lipopolysaccharide-induced tumor necrosis factor-alpha, IL-6 and IL-8 release by human monocytes and alveolar macrophages. J Immunol 1993; 150(7):2885-91.

27. Derikx JP, Poeze M, van Bijnen AA, et al. Evidence for intestinal and liver epithelial cell injury in the early phase of sepsis. Shock 2007; 28(5):544-8.

28. Laschinger JC, Cunningham JN, Jr., Nathan IM, et al. Experimental and clinical assessment of the adequacy of partial bypass in maintenance of spinal cord blood flow during operations on the thoracic aorta. Ann Thorac Surg 1983; 36(4):417-26.

29. Kazui T, Yamada O, Ito T, et al. [Total graft replacement of the thoracoabdominal aorta with reconstruction of visceral branches, intercostal and lumbar arteries in expanding chronic dissecting aneurysms of the thoracoabdominal aorta]. Nippon Kyobu Geka Gakkai Zasshi 1989; 37(7):1436-40.

30. Bailey RW, Bulkley GB, Hamilton SR, et al. Pathogenesis of nonocclusive ischemic colitis. Ann Surg 1986; 203(6):590-9.

31. Krejci V, Hiltebrand LB, Erni D, Sigurdsson GH. Endothelin receptor antagonist bosentan improves microcirculatory blood flow in splanchnic organs in septic shock. Crit Care Med 2003; 31(1):203-10.

32. Sautner T, Wessely C, Riegler M, et al. Early effects of catecholamine therapy on mucosal integrity, intestinal blood flow, and oxygen metabolism in porcine endotoxin shock. Ann Surg 1998; 228(2):239-48.

33. Gunel E, Caglayan F, Caglayan O, et al. Treatment of intestinal reperfusion injury using antioxidative agents. J Pediatr Surg 1998; 33(10):1536-9.

34. Udassin R, Haskel Y, Samuni A. Nitroxide radical attenuates ischaemia/reperfusion injury to the rat small intestine. Gut 1998; 42(5):623-7.

35. Idu MM, Heintjes RJ, Scholten EW, et al. Visceral and renal tissue oxygenation during supraceliac aortic crossclamping and left heart bypass with selective organ perfusion. Eur J Vasc Endovasc Surg 2004; 27(2):138-44.

36. Leijdekkers VJ, Wirds JW, Vahl AC, et al. The visceral perfusion system and distal bypass during thoracoabdominal aneurysm surgery: an alternative for physiological blood flow? Cardiovasc Surg 1999; 7(2):219-24. 


\subsection{New insight in loss of gut barrier during major non- abdominal surgery}

Joep P. M. Derikx, Dick A. van Waardenburg, Geertje Thuijls,

Henriëtte M. Willigers, Marianne Koenraads, Annemarie A. van Bijnen,

Erik Heineman, Martijn Poeze, Ton Ambergen, André van Ooij, Lodewijk W. van Rhijn, Wim A. Buurman

PLOS ONE, (2008) 3: e3954. 


\section{ABSTRACT}

\section{Background}

Gut barrier loss has been implicated as a critical event in the occurrence of postoperative complications. We aimed to study the development of gut barrier loss in patients undergoing major non-abdominal surgery.

\section{Methodology/Principal Findings}

Twenty consecutive children undergoing spinal fusion surgery were included. This kind of surgery is characterized by long operation time, significant blood loss, prolonged systemic hypotension, without directly leading to compromise of the intestines by intestinal manipulation or use of extracorporeal circulation. Blood was collected preoperatively, every two hours during surgery and 2, 4, 15 and 24 hours postoperatively. Gut mucosal barrier was assessed by plasma markers for enterocyte damage (I-FABP, I-BABP) and urinary presence of tight junction protein claudin-3. Intestinal mucosal perfusion was measured by gastric tonometry $\left(\mathrm{P}_{\mathrm{r}} \mathrm{CO}_{2}, \mathrm{P}_{\mathrm{r}-\mathrm{a}} \mathrm{CO}_{2}\right.$-gap).

Plasma concentration of I-FABP, I-BABP and urinary expression of claudin-3 increased rapidly and significantly after the onset of surgery in most children. Postoperatively, all markers decreased promptly towards baseline values together with normalisation of MAP. Plasma levels of I-FABP, I-BABP were significantly negatively correlated with MAP at $1 / 2$ hour before blood sampling $(-0.726(\mathrm{p}<0.001),-0.483(\mathrm{P}<0.001)$, respectively). Furthermore, circulating I-FABP correlated with gastric mucosal $\mathrm{P}_{\mathrm{r}} \mathrm{CO}_{2}, \mathrm{P}_{\mathrm{r}}$-aCO -gap measured at the same time points $(0.553(p=0.040), 0.585 \quad(p=0.028)$, respectively).

\section{Conclusions/Significance}

This study shows the development of gut barrier loss in children undergoing major non-abdominal surgery, which is related to preceding hypotension and mesenterial hypoperfusion. These data shed new light on the potential role of peroperative circulatory perturbation and intestinal barrier loss. 


\section{INTRODUCTION}

Patients undergoing major surgery or sustaining severe trauma are at risk of developing morbidity and mortality from postoperative or posttraumatic systemic inflammatory response syndrome (SIRS), sepsis and multiple organ failure (MOF). The development of such potentially lethal complications in relatively healthy surgical or trauma patients is poorly understood ${ }^{1,2}$. Moreover, few human studies investigated the hypothesis, generated from animal studies, that the intestines are central in the origin of postoperative and posttraumatic sequelae ${ }^{3-5}$. Major surgery accompanied by systemic hypotension and blood loss is thought to lead to redistribution of blood to preserve the vital organs (brain and heart) at the expense of the splanchnic circulation ${ }^{3-5}$. Low mesenteric blood flow subsequently leads to injury of the cells at the most distal point from the mucosal blood supply, being the mature enterocytes ${ }^{6}$.

Experimental animal models, resembling the clinical situation of major surgery and trauma, show that haemorrhagic shock leads to disruption of the gut barrier, measured by elevated circulating levels of Fatty Acid Binding Proteins (FABP), originating from damaged intestinal epithelial cells and derangement of tight-junctions ${ }^{7,8}$. Moreover, translocation of macromolecules, microbial products and microbiota from the intestinal lumen to the circulation and mesenteric lymph nodes, spleen and liver occur ${ }^{5,7}$. The inflammatory response to translocated microbial products as endotoxin has been reported to be induced via various innate immune mechanisms, ranging from Toll Like Receptors to complement activation ${ }^{9,10}$.

Studies in patients undergoing major gastro-intestinal, cardiac or vascular surgery, investigating the role of the gut in the development of postoperative complications, are largely restricted to data on increased intestinal permeability for sugars, 51Cr-EDTA and the circulatory levels of endotoxin ${ }^{11-19}$. Several authors report changes in these parameters in patients following major surgery, indicating that the gut barrier is injured ${ }^{11-14}$. However, other reports using these tests lack to support these data ${ }^{15-18}$. Moreover, the value of measuring gut barrier with the use of sugar absorption probes is argued ${ }^{19}$. In conclusion, the debate regarding the involvement of the gut in patients undergoing major surgery is still open.

Plasma and urinary markers are currently available as useful non-invasive tools to study the condition of enterocytes and tight-junctions (TJ), the two components comprising the gut mucosal barrier. Enterocyte damage was assessed using plasma levels of Intestinal-Fatty Acid Binding Protein (I-FABP), a small cytosolic, water-soluble protein, primarily limited to mature enterocytes of small and large intestine ${ }^{20}$. I-FABP plasma levels rise rapidly after episodes 


\section{Chapter 4}

of acute intestinal ischaemia and inflammation ${ }^{20-22}$. Next, also circulating levels of Ileal-Bile Acid Binding Protein (I-BABP), which is exclusively present in mature enterocytes of the jejunum and ileum, were assessed ${ }^{23}$. TJ between neighbouring enterocytes are an important constituent of the intestinal epithelial barrier ${ }^{24}$. The transmembrane TJ protein Claudin-3, the essential sealing protein, disappears rapidly from the TJ following haemorrhagic shock and is released into the urine (own unpublished data).

The principal aim of this study was to investigate whether major nonabdominal surgery leads to intestinal barrier loss.

\section{Methods}

\section{Study design and patients}

This is a prospective clinical observational study in children undergoing spinal fusion surgery because of scoliosis in the University Hospital Maastricht between March 2006 and October 2007. Major spinal fusion surgery is characterized by long operation time, significant blood loss, prolonged systemic hypotension and the potential development of postoperative complications ${ }^{25}$. This type of surgery was chosen because it does not directly compromise the intestines by intestinal manipulation or the use of extracorporeal circulation ${ }^{25}$.

Informed, written consent was obtained by all patients or both parents/ caretakers whose information was used in the study prior to inclusion; the study was conducted with approval from the local medical ethical committee.

\section{Surgical procedures}

\section{Preoperative preparation and anaesthesia}

Anaesthesia was induced and maintained with either a volatile based technique with sevoflurane or an intravenous technique with propofol, combined with an opioid and a non-depolarising muscle relaxant. Sensory evoked potentials were monitored in patients at risk for spinal cord problems during surgery. According to the hospital protocol an intravenous technique with propofol was used in these patients. All patients were intubated and ventilator settings were adjusted to obtain normocapnia. Each patient had a forced-air warming system and all intravenous fluids were warmed to prevent hypothermia.

In addition to standard monitoring an arterial line was inserted into a radial artery to measure arterial pressure and to sample arterial blood, and a catheter was introduced into the bladder to measure urine output. Bloodsoaked gauzes were weighted as they were passed off the surgical field and the blood content of the cell saver was measured to measure blood loss. 
Perioperative fluid therapy was adapted to the individual patient with the aim to keep the patient normovolaemic throughout the operation. Isotonic crystalloids were used for maintenance and third space losses. Blood loss was replaced 1:1 with blood or colloid or 3:1 with crystalloids. Fluid administration was guided by calculation of maintenance and third space losses, blood loss, the arterial blood pressure, and haemoglobin values. There was no protocol to keep the blood pressure above or below a certain value.

\section{Surgery}

All the operations were performed by 2 senior spine surgeons (LvR and AvO) using three fusion approaches: posterior spinal fusion (PSF), anterior spinal fusion (ASF), and combined anterior and posterior fusion. The decision regarding the preferred fusion was made based on curve location, aetiology, rigidity, and the child's age, according to the current standards of scoliosis operative repair.

In PSF, the patient was positioned prone on padded chest rolls, rolled blanket bolster, or a Wilson spinal frame to provide adequate cushioning for the chest and abdomen while allowing vacant space preventing abdominal pressure. The skin was incised in a straight line over the vertebrae to be fused. Following osteotomy of all the spinous processes and facets included at the fusion area, the vertebrae were instrumented with combinations of pedicle-screws and hooks (CD Horizon Legacy 5.5 or 4.5 spinal systems, Medtronic, Heerlen, the Netherlands). Curve correction was performed with a combination of derotation and compression-distraction manoeuvres, and, if necessary, also by in situ bending of the rods. In ASF, the patient was placed on the operating table in the lateral position. The approach is from the convex scoliotic side. A thoracoabdominal approach, which included a split of the diaphragm near its insertion, retroperitoneal approach, or lateral intrathoracic approach was performed through the side of the curve convexity, with the patient lying on his side. Following exposure of the vertebrae, the involved discs and ribs were excised and the segmental vessels ligated or preserved. In all cases of combined fusion, single-staged procedures were carried out.

CD Horizon Legacy 5.5 or 4.5 spinal systems were used depending on age and weight of the patient. In case of anterior instrumentation CD Horizon Eclipse spinal system was used (Medtronic). No drains were used.

\section{Postoperative care}

At the end of the surgery, all the children were transferred to the paediatric Intensive Care Unit (ICU). Extubation was performed after stabilization of vital signs and according to accepted weaning parameters (usually $4-6$ hours after surgery). Paediatric ICU management was provided by the attending physicians 


\section{Chapter 4}

guided by the same general management strategy and consisted of intravenous fluid administration, correction of hypovolaemia, electrolyte disturbances and/or anaemia and analgesics (acetaminophen and morphine). Antibiotic cover (amoxicillin with clavulanate) was given starting preoperatively until 24 hours after the end of surgery. Oral feeding was introduced the day after surgery.

Follow-up in the ICU included a daily physical examination, vital signs monitoring, routine blood tests, and chest radiographs or other ancillary tests as required. The attending physicians recorded complications and events.

\section{Blood and urine sampling}

Blood samples were collected from the arterial line in pre-chilled EDTA vacuum tubes (BD vacutainer, Becton Dickinson Diagnostics, Aalst, Belgium) and kept on ice. Blood was centrifuged at $4{ }^{\circ} \mathrm{C}, 4000 \times \mathrm{G}$ for 15 minutes. Plasma was immediately stored in aliquots at $-80^{\circ} \mathrm{C}$ until analysis. Blood was sampled before surgery (after the induction of anaesthesia), at 2 hours intervals during surgery and 2, 4, 15 and 24 hours postoperatively from the arterial line.

Fresh specimens of urine were collected from the urinary bladder catheter, kept on ice and then frozen at $-80^{\circ} \mathrm{C}$ in aliquots within 2 hours of collection. Urine was collected every 20 minutes in the first 2 hours during surgery and thereafter at the same moments as blood was sampled.

\section{Measurements of FABP and claudin-3}

Plasma concentrations of I-FABP were determined using a highly specific commercially available enzyme-linked immunosorbent assay (ELISA) that selectively detects human I-FABP (standard: 20-5,000 pg/ml), kindly provided by Hycult Biotechnology (Uden, the Netherlands) and I-BABP (standard: 0.32$5 \mathrm{ng} / \mathrm{ml}$ ) as previously described ${ }^{26}$.

Claudin-3 urine levels were analyzed by western blotting. Equal amounts of each sample (adjusted to urinary creatinine levels) were separated by SDS-PAGE gel, transferred to PVDF-membrane and probed using primary antibody to claudin-3 (Rabbit anti-claudin-3 (34-1700), Zymed Laboratories, San Francisco, CA). After incubation with goat anti rabbit HRP-conjugated secondary antibody (Jackson, West Grove, PA), signal was detected by supersignal west pico chemiluminescence substrate (Pierce, Etten-Leur, the Netherlands). Band intensity was semi-quantitatively analyzed using Quantity One (Biorad, Hercules, CA).

\section{Measurements of gastric mucosal tonometry}

A gastric tonometry catheter (14F, Datex Ohmeda, Helsinki, Finland) was introduced for measurement of intramucosal carbon dioxide pressure $\left(\mathrm{P}_{\mathrm{r}} \mathrm{CO}_{2}\right.$ in 
$\mathrm{kPa}$ ) throughout the surgical procedure in the last seven patients, using the gasautomated capnograph (Tonocap TC-200, Datex-Ohmeda).

Gastric tonometry measurements $\left(\mathrm{P}_{\mathrm{r}} \mathrm{CO}_{2}\right.$, and mucosal-arterial $\mathrm{pCO}_{2}$ gap $\left(\mathrm{P}_{\mathrm{r}-\mathrm{a}} \mathrm{CO}_{2}\right.$-gap)) were measured at 30-minute intervals from the start until the end of surgery using gas-automated capnography. The $\mathrm{pCO}_{2}$ values of the blood gases were corrected for the central blood temperature measurements, using the formulas provided by the manufacturer (ABL 100, Radiometer, Copenhagen, Denmark).

\section{Statistical analysis}

Statistical analysis was performed with Prism 4.0 for Windows (GraphPad Software Inc. San Diego, CA) and SPSS 15.0 (SPSS, Inc., Chicago, IL). Plasma FABP concentrations were presented as mean \pm standard error (SEM). Normality of all data obtained was verified by Kolmogorov-Smirnov test.

Linear mixed model regression was used to analyze changes over time in plasma FABP levels. Within-person correlations between mean arterial pressure (MAP), $\mathrm{P}_{\mathrm{r}} \mathrm{CO}_{2} / \mathrm{CO}_{2}$-gap and circulating levels of FABP at all studied time points were computed. Mixed model analysis accounts for unbalanced numbers of samples measured, because at some time points not all samples could be obtained because of removal of the arterial line. Differences in plasma FABP levels between individual time periods were compared using $t$ tests of the mixed model procedure. Next, to characterize the total amount of intestinal mucosal cell injury during surgery, area under the curve (AUC) for I-FABP and I-BABP was calculated for each patient. The $A \cup C_{\text {I-FABP }}$ and $A \cup C_{\text {I-BABP }}$ for patients with and without early complications was compared using unpaired $t$ test. Early complications were defined as complications occurring in the intraoperative or initial hospitalization period.

A p-value below 0.05 was considered to be statistically significant.

\section{Results}

\section{Patients}

Twenty patients undergoing spinal fusion surgery were consecutively included in the study, 15 girls and 5 boys. Median age was 12 years (range: 2-16 years). Demographic, surgical and fluid balance data are presented in Table 1. Intraoperative fluid resuscitation was adequate as evidenced by; 1 ) a mean (SEM) positive fluid balance (total fluid in minus blood loss) of 13 (1) $\mathrm{ml} / \mathrm{kg} / \mathrm{hr}$; 2) adequate diuresis; 3) low plasma lactate levels and; 4) adequate plasma haemoglobin value (data not shown). 
Table 1: Demographic, surgical and fluid balance characteristics.

\begin{tabular}{|c|c|c|c|c|c|}
\hline $\mathrm{No}^{0}$ & $\begin{array}{l}\text { AGe } \\
\text { (y) }\end{array}$ & $\begin{array}{l}\text { Weight } \\
(\mathrm{kg})\end{array}$ & Surgery' & $\begin{array}{l}\text { Duration } \\
\text { surgery (hr) }\end{array}$ & History² \\
\hline 1 & 8 & 36 & ASF T7-L4 & 6 & Spina bifida hydrocephalus \\
\hline 2 & 15 & 40 & $\begin{array}{l}\text { ASF+PSF } \\
\text { T3-S1 }\end{array}$ & 8 & $\begin{array}{l}\text { cerebral palsy, } \\
\text { Spastic diplegia,IVH }\end{array}$ \\
\hline 3 & 12 & 51 & ASF+PSF T12-L3 & 8 & - \\
\hline 4 & 12 & 31 & $\begin{array}{l}\text { ASF+PSF } \\
\text { T2-S1 }\end{array}$ & 9 & $\begin{array}{l}\text { DiGeorge syndrome; } \\
\text { Vascular spinal cord lesion }\end{array}$ \\
\hline 5 & 13 & 59 & PSF T4-L3 & 6 & cleft lip nose deformity \\
\hline 6 & 11 & 37 & PSF T3-T12 & 4 & spina bifida occulta \\
\hline 7 & 8 & 45 & PSF T2-L5 & 4 & spinal muscular atrophy \\
\hline 8 & 10 & 48 & ASF T6-T12 & 4 & - \\
\hline 9 & 14 & 33 & PSF T3-S1 & 8 & $\begin{array}{l}\text { spastic tetraplegia, } \\
\text { panencephalitis }\end{array}$ \\
\hline 10 & 15 & 55 & ASF T6-T12 & 4 & - \\
\hline 11 & 2 & 9 & ASF T12-L2 & 2 & $\begin{array}{l}\text { Conradi-Hünermann } \\
\text { Happle syndrome }\end{array}$ \\
\hline 12 & 10 & 22 & $\begin{array}{l}\text { ASF+PSF } \\
T 2-S 1\end{array}$ & 7 & $\begin{array}{l}\text { cerebral palsy, } \\
\text { spastic tertraparesis }\end{array}$ \\
\hline 13 & 15 & 82 & $\begin{array}{l}\text { ASF+PSF } \\
\text { T3-L3 }\end{array}$ & 7.5 & - \\
\hline 14 & 16 & 54 & ASF T7-T12 & 4 & - \\
\hline 15 & 13 & 20 & PSF T2-S1 & 8 & $\begin{array}{l}\text { spina bifida; } \\
\text { Arnold Chirari } \\
\text { Type } 2 \text { malformaiton }\end{array}$ \\
\hline 16 & 13 & 14 & PSF T3-L4 & 4 & Ullrich disease \\
\hline 17 & 9 & 16 & PSF T3-L4 & 5 & $\begin{array}{l}\text { Pierre Robin } \\
\text { Sequence; acampolic } \\
\text { campomelic dysplasia }\end{array}$ \\
\hline 18 & 12 & 52 & PSF T6-L1 & 5 & - \\
\hline 19 & 16 & 55 & PSF T5-L4 & 6.5 & - \\
\hline 20 & 12 & 34 & PSF L4-S1 & 6 & spondylolisthesis \\
\hline
\end{tabular}

0 No: patient number in sequence of entrance to the study

1 ASF: anterior spinal fusion; PSF: posterior spinal fusion

2 IVH: intraventricular haemorrhage 


\begin{tabular}{|c|c|c|c|c|c|}
\hline $\begin{array}{l}\text { Early } \\
\text { complications }{ }^{3}\end{array}$ & $\begin{array}{l}\text { Blood loss } \\
(\mathrm{ml} / \mathrm{kg})^{4}\end{array}$ & $\begin{array}{l}\text { Fluid in } \\
(\mathrm{m} / / \mathrm{kg})^{4}\end{array}$ & $\begin{array}{l}\text { Diuresis } \\
(\mathrm{ml} / \mathrm{kg} / \mathrm{hr})^{4}\end{array}$ & $\begin{array}{l}\text { Mean (range) } \\
\operatorname{MAP}(\mathrm{mmHg})^{4}\end{array}$ & $\begin{array}{l}\text { Mean (range) } \\
\text { lactate }(\mathrm{mmol} / /)^{4}\end{array}$ \\
\hline \multirow[t]{2}{*}{ fever, UTI } & 14 & 103 & 3.5 & $57(52-63)$ & $1.0(0.8-1.2)$ \\
\hline & 20 & 131 & 1.7 & $54(41-66)$ & $1.2(1.0-1.3)$ \\
\hline fever, pneumonia & 59 & 163 & 1.1 & $56(50-66)$ & $1.9(1.6-2.6)$ \\
\hline melaena & 32 & 190 & 1.5 & $59(45-77)$ & $1.6(0.8-1.9)$ \\
\hline - & 53 & 156 & 2.5 & $65(56-70)$ & $1.3(1.2-1.4)$ \\
\hline - & 11 & 95 & 1.5 & 81 (70-95) & $1.8(1.6-2.1)$ \\
\hline - & 14 & 68 & 1.9 & $55(51-60)$ & $0.9(0.7-1.1)$ \\
\hline- & 13 & 73 & 4.2 & $66(59-75)$ & $1.5(1.2-1.9)$ \\
\hline - & 91 & 341 & 5.3 & $65(51-80)$ & $1.2(0.8-1.5)$ \\
\hline - & 5 & 109 & 1.2 & 79 (70-101) & $2.6(1.6-3.5)$ \\
\hline - & 1 & 34 & 0.3 & $58(51-70)$ & $1.4(1.1-1.6)$ \\
\hline - & 9 & 203 & 1.3 & $50(39-56)$ & $1.3(1.1-1.5)$ \\
\hline $\begin{array}{l}\text { Fever, wound } \\
\text { infection }\end{array}$ & 30 & 103 & 2.5 & $64(53-88)$ & $1.1(0.9-1.2)$ \\
\hline- & 4 & 56 & 0.8 & $71(63-82)$ & $0.8(0.6-1.3)$ \\
\hline $\begin{array}{l}\text { peroperative } \\
\text { anaphylactic } \\
\text { shock to Venofundin }\end{array}$ & 75 & 180 & 1.0 & $53(33-78)$ & $0.9(0.8-1.1)$ \\
\hline- & 41 & 129 & 0.3 & $59(41-72)$ & $0.7(0.5-0.8)$ \\
\hline - & 6 & 94 & 1.1 & $67(57-93)$ & $1.1(0.9-1.4)$ \\
\hline UTI & 6 & 58 & 2.8 & $68(60-81)$ & $0.9(0.7-1.1)$ \\
\hline- & 40 & 123 & 1.3 & $70(58-87)$ & $0.7(0.6-1.0)$ \\
\hline - & 18 & 103 & 0.9 & $56(49-73)$ & $0.8(0.7-0.9)$ \\
\hline
\end{tabular}

${ }^{3}$ UTI: urinary tract infection

${ }^{4}$ these parameters are measured intra-operatively

y: years, kg: kilograms, hr: hours, MAP: mean arterial pressure. 


\section{Chapter 4}

\section{Plasma I-FABP, I-BABP}

The plasma concentration of I-FABP increased rapidly after the initiation of surgery from a mean (SEM) baseline value of 221 (32) pg/ml shortly before start of surgery, under anaesthesia (in-house mean normal value: $106 \mathrm{pg} / \mathrm{ml}$, range: $41-336 \mathrm{pg} / \mathrm{ml})$ to $348(44) \mathrm{pg} / \mathrm{ml}$ at 2 hours after the onset of surgery $(\mathrm{p}=0.006)$ (Figure 1a). Thereafter, the mean plasma levels increased further to 369 (33) $\mathrm{pg} / \mathrm{ml}(\mathrm{p}<0.001)$ at 4 hours after initiation of surgery. The peak value of $443(69)$ $\mathrm{pg} / \mathrm{ml}(\mathrm{p}<0.001)$ was reached at 6 hours after the start of surgery, which often represented the end of surgery. Thirteen patients showed an increase in plasma I-FABP levels of at least twofold during surgery; while 7 patients had relatively unchanged circulating I-FABP values. Plasma concentrations of I-FABP decreased towards baseline values from 2 hours after the end of surgery onwards.

Similar to the I-FABP levels, mean I-BABP plasma concentrations also increased significantly between 2 and 8 hours after start of surgery compared to baseline values in most of the patients (Figure $1 \mathrm{~b}$ ).

Since FABP are excreted by the kidneys, we evaluated whether high plasma values of FABP could be caused by impaired renal function. Diuresis during and after surgery was adequate (Table 1 ) and plasma creatinine values were not elevated, which indicates that elevation of plasma FABP was caused by enterocyte cell death.
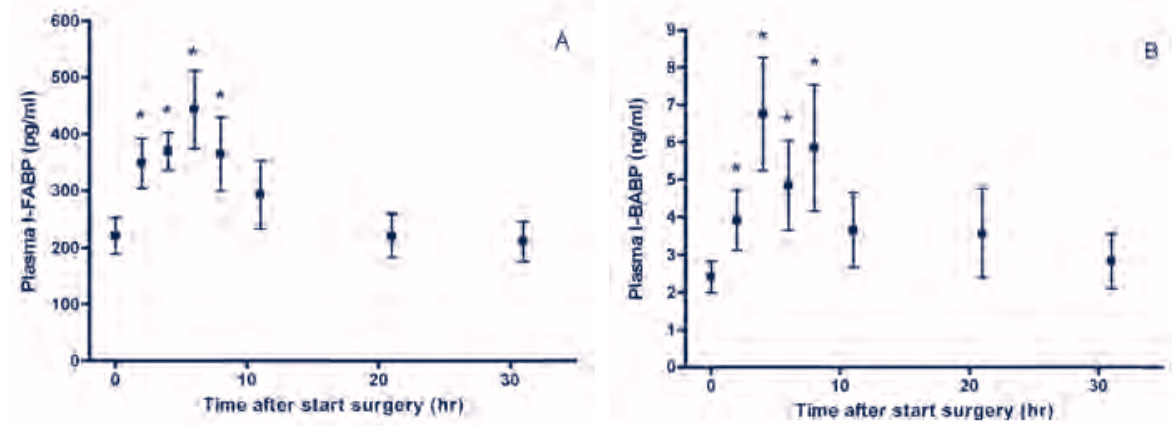

Figure 1: Time course of mean (SEM) plasma I-FABP (a) and I-BABP (b) levels in children undergoing spinal fusion surgery $(n=20)$.

$* \mathrm{p}<0.05$ vs. baseline values. 


\section{Urinary claudin-3}

The urinary claudin-3:creatinine ratio immediately increased during the first 20 minutes of surgery. In the next 2 hours, the claudin-3:creatinine ratio remained high and thereafter a decrease towards preoperative values was detected (Figure 2).

\section{Patient 18}
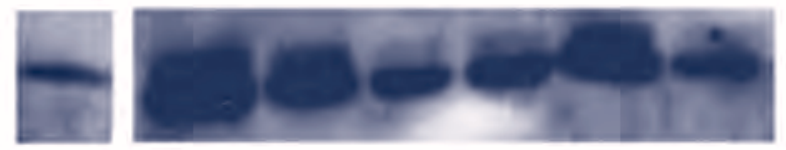

$22 \mathrm{kDa}$

Patient 20
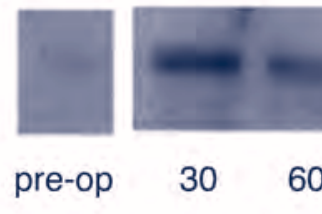

Figure 2: Time course of urinary claudin-3:creatinine ratio of two representative of the last five children undergoing spinal fusion surgery.

\section{Gastric tonometry and mean arterial pressure in relation to intestinal damage}

The very short circulating half-life of FABP (approximately 11 minutes) ${ }^{27}$ allows to relate the presence of enterocyte cell damage with preceding systemic hypotension and gastric mucosal hypoperfusion. To this end within-person correlations were studied between circulating levels of I-FABP, I-BABP and intraoperative MAP at $1 / 2$ hour before the blood sample was collected in which FABP concentration was measured, and $\mathrm{P}_{\mathrm{r}} \mathrm{CO}_{2}, \mathrm{P}_{\mathrm{r}-\mathrm{a}} \mathrm{CO}_{2}$-gap at the same moment of blood sampling. Interestingly, plasma levels of I-FABP, I-BABP were significantly negatively correlated with MAP at $1 / 2$ hour before blood sampling (correlation: $-0.726(\mathrm{p}<0.001)$; $-0.483(\mathrm{P}<0.001)$, respectively), indicating a relationship between enterocyte cell damage and preceding systemic hypotension (Figure 3, Table 2). Furthermore, circulating values of I-FABP correlated with gastric mucosal $\mathrm{P}_{\mathrm{r}} \mathrm{CO}_{2}$ and $\mathrm{P}_{\mathrm{r}-\mathrm{C}} \mathrm{CO}_{2}$-gap measured at the same time points (correlation: $0.553(\mathrm{p}=0.040)$ and $0.585(\mathrm{p}=0.028)$, respectively), whereas no correlation was observed between plasma levels of I-BABP and $\mathrm{P}_{\mathrm{r}} \mathrm{CO}_{2}$ or $\mathrm{P}_{\mathrm{r}-\mathrm{a}} \mathrm{CO}_{2}$-gap. These data show a clear association between the most prominent plasma marker for enterocyte cell death (I-FABP), hypotension and splanchnic hypoperfusion, assessed by gastric mucosal $\mathrm{P}_{\mathrm{r}} \mathrm{CO}_{2}$ and $\mathrm{P}_{\mathrm{r}-\mathrm{a}} \mathrm{CO}_{2}$-gap. 


\section{Chapter 4}

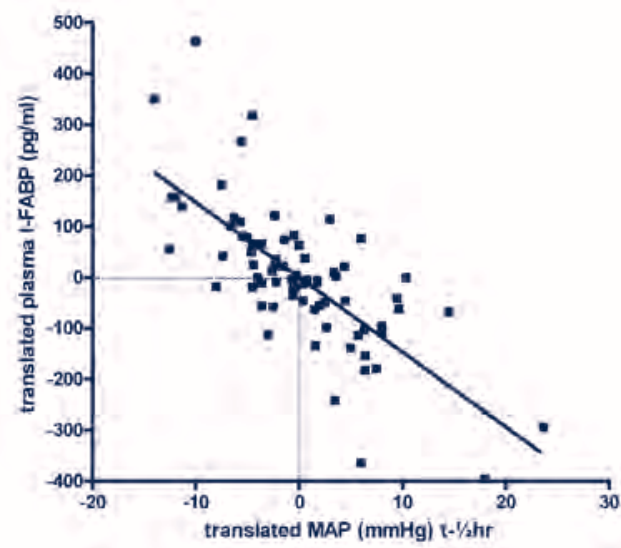

Figure 3: Translated values of plasma levels of circulating I-FABP and translated values of preceding systemic hypotension (MAP t-1/2hr) in children undergoing spinal fusion surgery were plotted. Circulating I-FABP correlated significantly negatively with MAP at 1/2 hour before blood sampling $(\mathrm{n}=89$, correlation: $-0.726(\mathrm{p}<0.001)$. Translations of both variables are specific for an individual in such a way that all within-person means correspond to the zeros in the plot. In this way the variation of individual levels are cancelled and the pure association of both variables remains.

Table 2: Within-person correlations between enterocyte cell damage and preceding systemic hypotension and gastric mucosal hypoperfusion.

\begin{tabular}{lccc} 
& MAP t-1/2 hr $(\mathrm{mmHg})$ & $\mathrm{P}_{\mathrm{r}} \mathrm{CO}_{2}(\mathrm{kPa})$ & $\mathrm{P}_{\mathrm{r}-\mathrm{a}} \mathrm{CO}_{2}$.gap $(\mathrm{kPa})$ \\
$\mathrm{N}$ & 89 & 32 & 32 \\
\hline I-FABP $(\mathrm{pg} / \mathrm{ml})$ & $-0.726(\mathrm{P}<0.001)$ & $0.553(\mathrm{P}=0.040)$ & $0.585(\mathrm{P}=0.028)$ \\
I-BABP $(\mathrm{ng} / \mathrm{ml})$ & $-0.483(\mathrm{P}<0.001)$ & $-0.051(\mathrm{P}=0.862)$ & $-0.079(\mathrm{P}=0.787)$ \\
\hline
\end{tabular}

Within-person correlations between enterocyte cell damage (plasma I-FABP and I-BABP) and preceding systemic hypotension (mean arterial pressure (MAP) at $1 / 2$ hour before collection of the blood sample for $\mathrm{FABP}$ assessment) and gastric mucosal hypoperfusion $\left(\mathrm{P}_{\mathrm{r}} \mathrm{CO}_{2}, \mathrm{P}_{\mathrm{r}-\mathrm{a}} \mathrm{CO}_{2-\mathrm{gap}}\right.$ at the same moment of blood sampling). $N=$ number of measurements.

\section{Complications}

Six patients had nine early complications, including postoperative fever $(n=3)$, urinary tract infection $(n=2)$, pneumonia $(n=1)$, wound infection $(n=1)$, peroperative anaphylactic reaction to poly $(\mathrm{O}-2$-hydroxyethyl)starch (Venofundin) $(n=1)$ and melaena of unknown origin $(n=1)$. 
The mean $A \cup C_{\text {I-FABP }}$ for the six patients with complications was $222 \mathrm{pg} * \mathrm{hr} / \mathrm{ml}$ (range: 0-493 $\mathrm{pg}^{*} \mathrm{hr} / \mathrm{ml}$ ), while for patients without complications the mean $A C_{\text {I-FABP }}$ was $81 \mathrm{pg} * \mathrm{hr} / \mathrm{ml}$ (range: 0-222 pg*hr/ml) (p=0.032) (Figure 4). No significant changes were found in mean $\mathrm{AUC}_{\mathrm{I-BABP}}$ during surgery between patients with and without complications (3.1 vs. $2.0 \mathrm{ng} * \mathrm{hr} / \mathrm{ml}, \mathrm{p}=0.341$ ).

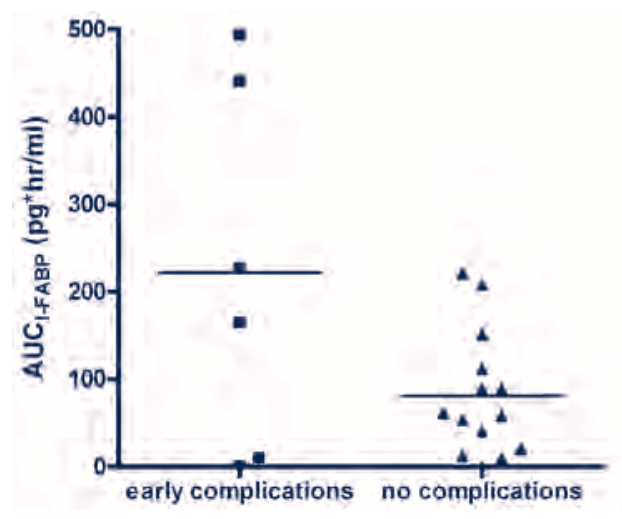

Figure 4: Area under the curve of plasma I-FABP values ( $\mathrm{U}_{\mathrm{I}} \mathrm{C}_{\mathrm{FABP}}$ ) during surgery for six patients with early complications and 14 patients without complications. Mean AUC I-FABP was significantly higher in patients with complications than in patients without complications $(p=0.032)$. The horizontal lines indicate the mean $A \cup C_{1-F A B P}$.

\section{Discussion}

The data showing an early increase of circulating FABP and urinary claudin-3, followed by rapid return towards baseline values, indicate that the patients suffered transient injury to the mature enterocytes and their tight junctions. Interestingly, similar kinetics of circulating FABP and urinary claudin-3 were found in all patients, regardless of extension of surgery or amount of blood loss. It remains to be established whether this insult is sufficient for a breakdown of the intestinal mucosal barrier, eliciting an inflammatory response and postoperative complications, as has been shown in animal studies ${ }^{5,8,28}$. Although this study was only set up to explore the development of intestinal mucosal cell damage during major non-abdominal surgery, a limited analysis of the postoperative course of patients with intestinal mucosal cell damage revealed that higher plasma levels of I-FABP were associated with a higher rate of postoperative complications. All possible complications are described as end point, because this type of surgery in relatively healthy children rarely results in important complications, including sepsis, MOF and death. Nevertheless, the 


\section{Chapter 4}

described complications were associated with prolonged hospitalization. Taken together, this study did not prove causality that the observed gut barrier loss and inflammation are the inducing factors for SIRS and MOF. Additional work, enrolling more patients, including those likely to experience serious complications, is needed in order to fully unravel the sequelae of the observed gut barrier loss.

Our work is supported by three previous studies showing the temporary presence of intestinal villous cell damage, measured by increased urinary levels of I-FABP, in patients undergoing cardiac surgery with cardiopulmonary bypass (CPB) ${ }^{29-31}$. In line, the patients with high urinary I-FABP levels developed postoperative gastro-intestinal complications ${ }^{29}$. The use of CPB was shown to be responsible for alterations in blood flow with consequently intestinal mucosal hypoxia and villous tip ischemia ${ }^{30,}{ }^{31}$. In our patients a similar influence of variation in blood flow on the provocation of intestinal villous cell injury was found, without the use of extracorporeal circulation.

Hypotension is often accepted to diminish blood loss and thereby facilitating surgical exposure and reducing the need for blood transfusions during e.g. oromaxillofacial, neurosurgery and major orthopaedic surgery ${ }^{32,} 33$. Peroperative hypotension is considered to be caused by hypovolaemia and/or anaesthetics. It is unlikely that the children undergoing spinal fusion repair were hypovolaemic, because fluids were administered adequately, which is reflected by positive fluid balance, low plasma lactate levels and sufficient diuresis. Therefore, anaesthetics are the major cause of low MAP. Propofol and sevoflurane, which were used in almost all children as anaesthetic agents have only minimal effects on cardiac output, but they decrease the systemic vascular resistance significantly, resulting in hypotension ${ }^{34}$. However, the fall in blood pressure together with prolonged surgery and anaemia potentially results in tissue hypoxia, represented by transient splanchnic hypoperfusion, impairment of hepatocellular integrity, renal dysfunction and visual loss because of optic nerve ischemia ${ }^{32,33,35}$. Our study shows for the first time the relation between accepted hypotension and the development of intestinal mucosal cellular damage in patients undergoing major non-abdominal surgery.

The clinical consequences of our findings are challenging. It is clear that major (non-abdominal) surgery, accompanied by accepted systemic hypotension aimed at minimizing intraoperative blood loss, can induce splanchnic mucosal hypoperfusion and gut barrier loss. While organ blood flow regulation is preserved over a wide range of MAP, organ perfusion becomes pressure dependent when the MAP decreases below a certain critical level (autoregulatory threshold). The autoregulatory threshold varies between different organs, the 
presence of diseases and age; little data are available on the autoregulatory threshold in children, and no studies report on the child intestinal autoregulation. We are currently performing a study in which haemodynamic optimization, aimed at normotension and flow-directed parameters, is intended during major surgery in order to prevent the development of intestinal damage.

The present study concerns relatively healthy children and young adolescents, who have an insignificant risk for important complications after major surgery. In line, studies with older patients undergoing surgery or trauma show that increasing age is one of the most crucial risk factors influencing adverse outcome ${ }^{36,37}$. Therefore, we speculate that the loss of the intestinal mucosal barrier as observed in our study in relatively healthy children undergoing major non-abdominal surgery would have a larger effect on the development of postoperative complications in older patients. Collectively, these findings shed a new light on the potential role of intestinal barrier compromise during major surgery, which was adapted from numerous animal studies, but now reported in relatively healthy children and adolescents undergoing major non-abdominal surgery. Furthermore, we consider that these results indicate a need to re-examine currently accepted criteria of haemodynamic parameters in patients undergoing major surgery.

\section{References}

1. Russell JA (2006) Management of sepsis. N Engl J Med 355: 1699-1713.

2. Rizoli SB, Marshall JC (2002) Saturday night fever: finding and controlling the source of sepsis in critical illness. Lancet Infect Dis 2: 137-144.

3. Fink MP, Delude RL (2005) Epithelial barrier dysfunction: a unifying theme to explain the pathogenesis of multiple organ dysfunction at the cellular level. Crit Care Clin 21: 177-196.

4. Moore FA (1999) The role of the gastrointestinal tract in postinjury multiple organ failure. Am J Surg 178: 449-453.

5. Rotstein OD (2000) Pathogenesis of multiple organ dysfunction syndrome: gut origin, protection, and decontamination. Surg Infect (Larchmt) 1: 217-223.

6. Ohri SK, Somasundaram S, Koak Y, Macpherson A, Keogh BE, et al (1994) The effect of intestinal hypoperfusion on intestinal absorption and permeability during cardiopulmonary bypass. Gastroenterology 106: 318-323.

7. Haan Jd, Lubbers T, Hadfoune M, Luyer M, Dejong C, et al (2008) Post-Shock Intervention with High-Lipid Enteral Nutrition Reduces Inflammation and Tissue Damage. Ann Surg. 


\section{Chapter 4}

8. Yang R, Han X, Uchiyama T, Watkins SK, Yaguchi A, et al (2003) IL-6 is essential for development of gut barrier dysfunction after hemorrhagic shock and resuscitation in mice. Am J Physiol Gastrointest Liver Physiol 285: G621-629.

9. Beutler B (2004) Inferences, questions and possibilities in Toll-like receptor signalling. Nature 430: 257-263.

10. Strunk RC, Whitehead AS, Cole FS (1985) Pretranslational regulation of the synthesis of the third component of complement in human mononuclear phagocytes by the lipid A portion of lipopolysaccharide. J Clin Invest 76: 985-990.

10. Quezado ZM, Hoffman WD, Winkelstein JA, Yatsiv I, Koev CA, et al (1994) The third component of complement protects against Escherichia coli endotoxin-induced shock and multiple organ failure. J Exp Med 179: 569-578.

11. Braun JP, Buhner S, Kastrup M, Dietz E, Langer K, et al (2007) Barrier function of the gut and multiple organ dysfunction after cardiac surgery. J Int Med Res 35: 72-83.

12. Holland J, Carey M, Hughes N, Sweeney K, Byrne PJ, et al (2005) Intraoperative splanchnic hypoperfusion, increased intestinal permeability, down-regulation of monocyte class II major histocompatibility complex expression, exaggerated acute phase response, and sepsis. Am J Surg 190: 393-400.

13. Riddington DW, Venkatesh B, Boivin CM, Bonser RS, Elliott TS, et al (1996) Intestinal permeability, gastric intramucosal $\mathrm{pH}$, and systemic endotoxemia in patients undergoing cardiopulmonary bypass. Jama 275: 1007-1012.

14. Soong CV, Halliday MI, Barclay GR, Hood JM, Rowlands BJ, et al (1997) Intramucosal acidosis and systemic host responses in abdominal aortic aneurysm surgery. Crit Care Med 25: 1472-1479.

15. Kanwar S, Windsor AC, Welsh F, Barclay GR, Guillou PJ, et al (2000) Lack of correlation between failure of gut barrier function and septic complications after major upper gastrointestinal surgery. Ann Surg 231: 88-95.

16. Buttenschoen K, Buttenschoen DC, Berger D, Vasilescu C, Schafheutle S, et al (2001) Endotoxemia and acute-phase proteins in major abdominal surgery. Am J Surg 181: 36-43.

17. Rossi M, Sganga G, Mazzone M, Valenza V, Guarneri S, et al (2004) Cardiopulmonary bypass in man: role of the intestine in a self-limiting inflammatory response with demonstrable bacterial translocation. Ann Thorac Surg 77: 612-618.

18. Malagon I, Onkenhout W, Klok G, van der Poel PF, Bovill JG, et al (2005) Gut permeability in paediatric cardiac surgery. Br J Anaesth 94: 181-185.

19. Bjarnason I, MacPherson A, Hollander D (1995) Intestinal permeability: an overview. Gastroenterology 108: 1566-1581.

20. Lieberman JM, Sacchettini J, Marks C, Marks WH (1997) Human intestinal fatty acid binding protein: report of an assay with studies in normal volunteers and intestinal ischemia. Surgery 121: 335-342. 
21. Kanda T, Fujii H, Tani T, Murakami H, Suda T, et al (1996) Intestinal fatty acid-binding protein is a useful diagnostic marker for mesenteric infarction in humans. Gastroenterology 110: 339-343.

22. Derikx JP, Matthijsen RA, de Bruïne AP, van Bijnen AA, Heineman E, et al (2008) Rapid reversal of human intestinal ischemia-reperfusion induced damage by shedding of injured enterocytes and reepithelialisation. PLOS ONE 3: e3482.

23. Watanabe K, Hoshi N, Tsuura Y, Kanda T, Fujita M, et al (1995) Immunohistochemical distribution of intestinal 15 kDa protein in human tissues. Arch Histol Cytol 58: 303-306.

24. Zeissig S, Burgel N, Gunzel D, Richter J, Mankertz J, et al (2007) Changes in expression and distribution of claudin 2, 5 and 8 lead to discontinuous tight junctions and barrier dysfunction in active Crohn's disease. Gut 56: 61-72.

25. Deyo RA (2007) Back surgery--who needs it? N Engl / Med 356: 2239-2243.

26. Derikx JP, Blijlevens NM, Donnelly JP, Fujii H, Kanda T, et al (2008) Loss of enterocyte mass is accompanied by diminished turnover of enterocytes after myeloablative therapy in haematopoietic stem cell transplant recipients. Ann Oncol.

27. van de Poll MC, Derikx JP, Buurman WA, Peters WH, Roelofs HM, et al (2007) Liver manipulation causes hepatocyte injury and precedes systemic inflammation in patients undergoing liver resection. World J Surg 31: 2033-2038.

28. Luyer MD, Greve JW, Hadfoune M, Jacobs JA, Dejong CH, et al (2005) Nutritional stimulation of cholecystokinin receptors inhibits inflammation via the vagus nerve. J Exp Med 202: 1023-1029.

29. Holmes JHt, Lieberman JM, Probert CB, Marks WH, Hill ME, et al (2001) Elevated intestinal fatty acid binding protein and gastrointestinal complications following cardiopulmonary bypass: a preliminary analysis. J Surg Res 100: 192-196.

30. Morariu AM, Loef BG, Aarts LP, Rietman GW, Rakhorst G, et al (2005) Dexamethasone: benefit and prejudice for patients undergoing on-pump coronary artery bypass grafting: a study on myocardial, pulmonary, renal, intestinal, and hepatic injury. Chest 128: 2677-2687.

31. Hanssen SJ, Derikx JP, Vermeulen Windsant IC, Heijmans JH, Koeppel TA, et al (2008) Visceral injury and systemic inflammation in patients undergoing extracorporeal circulation during aortic surgery. Ann Surg 248: 117-125.

32. Degoute CS (2007) Controlled hypotension: a guide to drug choice. Drugs 67: 1053-1076.

33. Dutton RP (2004) Controlled hypotension for spinal surgery. Eur Spine J 13: S66-71.

34. Akata T (2007) General anesthetics and vascular smooth muscle: direct actions of general anesthetics on cellular mechanisms regulating vascular tone. Anesthesiology 106: 365-391.

35. Suttner SW, Boldt J, Schmidt CC, Piper SN, Schuster P, et al (1999) The effects of sodium nitroprusside-induced hypotension on splanchnic perfusion and hepatocellular integrity. Anesth Analg 89: 1371-1377. 


\section{Chapter 4}

36. Hannan EL, Racz MJ, Walford G, Ryan TJ, Isom OW, (2003) Predictors of readmission for complications of coronary artery bypass graft surgery. Jama 290: 773-780.

37. Khuri SF, Henderson WG, DePalma RG, Mosca C, Healey NA, et al (2005) Determinants of long-term survival after major surgery and the adverse effect of postoperative complications. Ann Surg 242: 326-341. 
Gut wall integrity loss in critical illness and surgery 


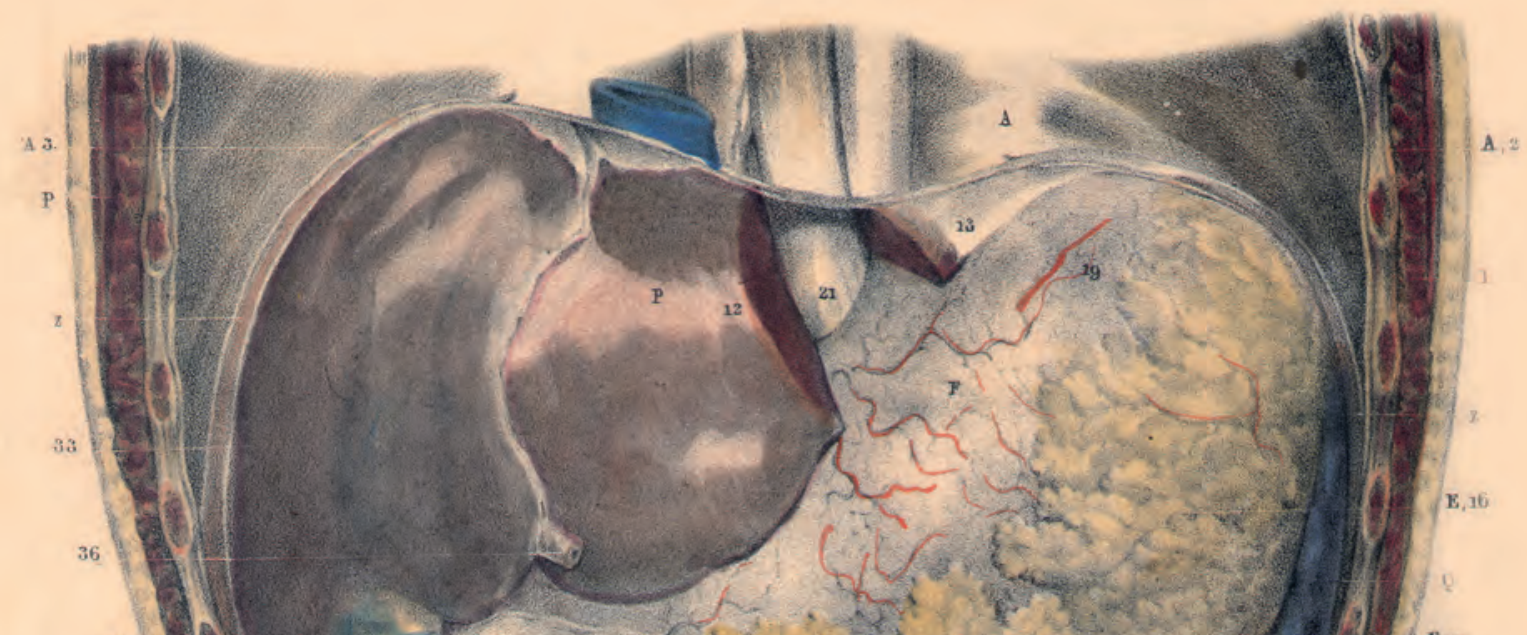

3510

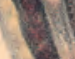

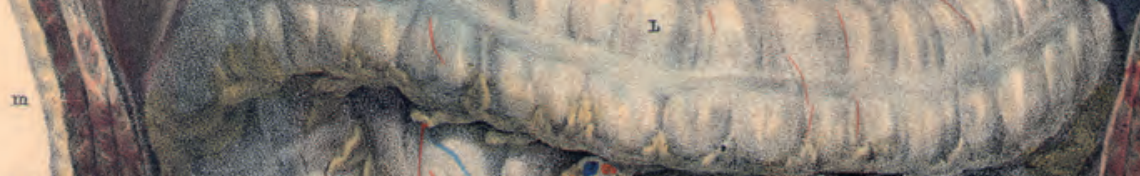

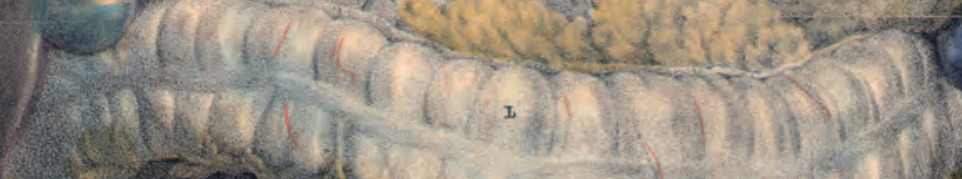

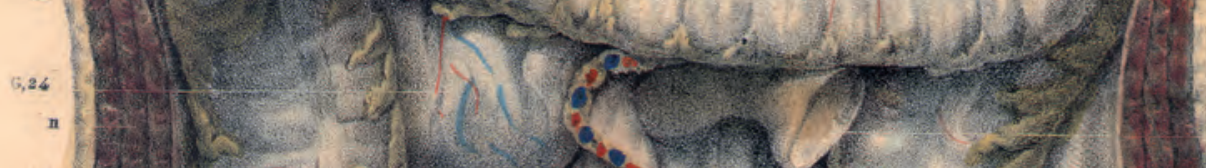

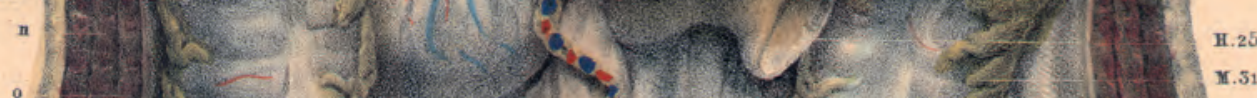

(1)

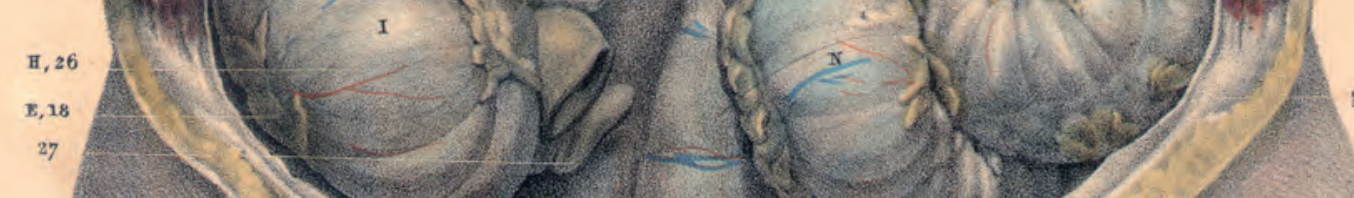

0

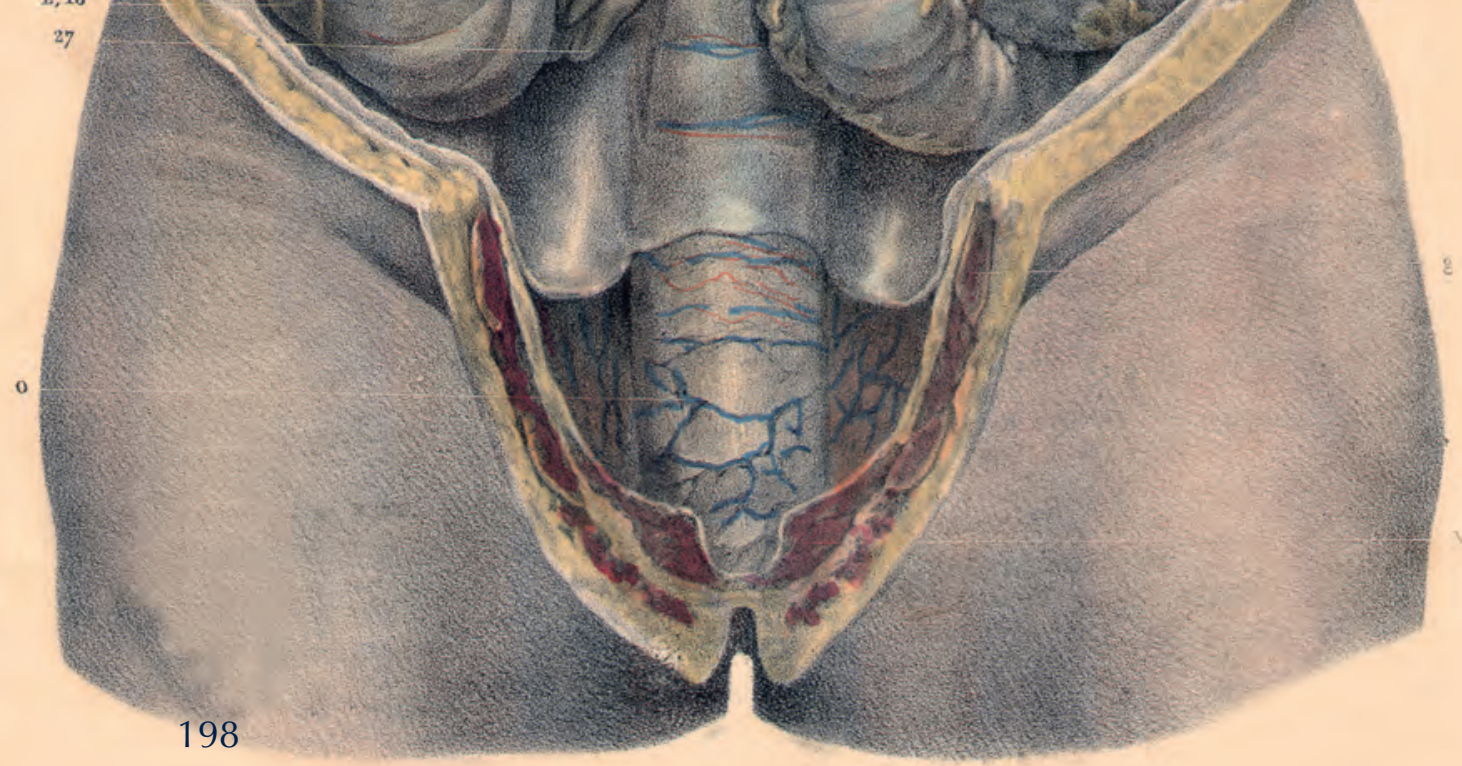




\section{ChaPter 5}

\section{Sequelae of human intestinal ischemia-reperfusion}




\section{Chapter 5}

\subsection{INTRODUCTION}

As altered splanchnic perfusion during major surgery and in critically ill patients was reported to be related to the development of gut wall integrity loss in earlier chapters, next aim was to set up a model, which made it possible to study the consequences of controlled intestinal ischemia-reperfusion (IR) in detail in man (paragraph 5.2). In addition, intestinal ischemia is not only encountered in critically ill patients or in patients undergoing major surgery, it is a disease entity itself (e.g. acute mesenteric ischemia), which is accompanied by high morbidity and mortality rates because of its aspecific presentation and the lack of accurate markers. Therefore, a human model for intestinal IR potentially would also provide the opportunity to study new markers. Finally, it needs to be brought forward that intestinal ischemia or hypoperfusion is a physiological condition occurring during exercise both in healthy relatively untrained volunteers and in highly trained athletes, which stresses the abdominal vasodilator reserve by diverting blood flow from the splanchnic circulation to the exercise muscles. This substantial reduction in blood flow is sometimes associated with symptoms of abdominal hypoperfusion, which are almost always reversible without intensive therapy. In conclusion, both physiological and pathophysioloical conditions are related with the development of intestinal ischemia. In the new human model to evaluate the effects of intestinal ischemia, the sequelae on the epithelial lining were first studied in paragraph 5.3. As animal studies suggested that the development of intestinal damage upon intestinal ischemia were dependent on Mannose-binding lectin (MBL) variations, an important initiating complement component, the amount of epithelial cell damage was related to MBL genotypes (paragraph 5.4). Finally, the relation between ischemically damaged intestinal epithelium and local inflammatory response was investigated in paragraph 5.5 using the newly developed human model to evaluate the consequences of intestinal ischemia-reperfusion. 


\subsection{A new model to study intestinal ischemia-reperfusion damage in man}

Joep P. M. Derikx *, Robert A. Matthijsen *, Adriaan P. de Bruïne, Annemarie A. van Bijnen, Ronald M. van Dam, Erik Heineman, Wim A. Buurman, Cornelis H. C. Dejong

* both authors contributed equally to this work. Submitted. 


\section{Chapter 5}

\section{ABSTRACT}

This report describes a human in vivo ischemia reperfusion (IR) model of the small intestine. Animal models of intestinal IR are indispensable for our understanding of sequelae of IR induced organ damage. However, a functional experimental IR model of the human small intestine, allowing for translational research, can be considered critical for our pathophysiological understanding of intestinal IR in man. Therefore patients with a healthy gut undergoing abdominal surgery with a Roux-Y or similar reconstruction were included, creating the opportunity to study IR of an isolated jejunal segment in a harmless model. Ischemia was induced by non-traumatic vascular clamping followed by reperfusion. This model can be adapted using variable ischemia and reperfusion times. Similarly tissue and plasma can be collected at any given time point during ischemia until end of reperfusion, only determined by progress of the original, intended surgical procedure. 


\section{INTRODUCTION}

Acute mesenteric ischemia refers to an acute onset of hypoperfusion of the intestine, often associated with major vascular or abdominal surgical procedures, small intestinal transplantation, major trauma and blood loss, sepsis and necrotizing enterocolitis (NEC) ${ }^{1-3}$. Intestinal ischemia followed by reperfusion (IR) complicates the treatment of critically ill patients. Furthermore, acute mesenteric ischemia is a disease entity in itself of which diagnosis remains difficult due to the lack of specific clinical markers ${ }^{4,5}$. Current diagnosis of acute mesenteric ischemia largely depends on clinical suspicion and is mainly aimed at exclusion of different less urgent pathologies presenting with similar symptoms. Intestinal IR has been linked to local as well as peripheral organ damage, the systemic inflammatory response syndrome (SIRS) and multiple organ dysfunction syndrome (MODS), accounting for up to $70 \%$ mortality ${ }^{1-3,6}$.

At present, our knowledge regarding mechanisms involved in IR induced intestinal damage has evolved from research performed in animal models that attempt to simulate human disease ${ }^{2,3,7,8}$. Different animal models have been developed in mice, rats, cats, dogs and pigs in which mainly ischemia of the small intestine is studied by transient clamping of the superior mesenteric artery. The use of animal models has identified important mechanisms contributing to IR induced organ damage. Indisputably the data from animal models have demonstrated the multifactorial etiology of IR damage in the intestine 2,3,8,9. However, differences exist between various animals and between strains of the same species regarding the results of intestinal ischemia 10,11 . Furthermore, early responses that drive human intestinal injury and organ failure in response to IR remain to be resolved. Therefore, we developed an experimental ischemia reperfusion model of the intestine in humans creating the possibility to address these points ${ }^{12}$.

Several determinants were important during model development: I) No additional invasive procedures should be needed to execute this model. II) A variable duration of ischemia and reperfusion should be possible. III) The possibility to collect serial and different (tissue/plasma) samples should be created. IV) The studied intestine should be healthy and not affected by prior inflammatory pathology. V) The small intestine should be studied. Considering these pre-determined demands a model was developed in patients that undergo major upper abdominal surgery with a Roux-Y or similar small intestinal reconstruction. We included patients with pylorus preserving pancreatico duodenectomy (PPPD) or Whipple's procedure, pancreatico jejunostomy, hepatico jejunostomy or Frey procedure. 


\section{Chapter 5}

The model described is comparable with currently existing animal models. Most animal models allow up to three hours of reperfusion after approximately 30 minutes of superior mesenteric artery clamping in order to induce ischemia and to study early alterations of the small intestine. The duration of the ischemic period, which is similarly induced by non-traumatic clamping of a superior mesenteric artery branch, is variable and followed by a chosen reperfusion time. The only determinant of the IR period is set by surgical boundaries imposed by surgical treatment of the patient. Additionally, it has to be kept in mind that besides ischemia reperfusion of the small intestine, intestinal hypoperfusion could similarly be studied using a comparable setup.

The surgery takes about 3-6 hours to complete and allows for a standardized ischemic period as well as reperfusion of up to 4 hours. Moreover, this model offers the possibility to collect various samples at different time points. Tissue undergoing reperfusion as well as healthy small intestinal tissue samples can be collected. The model even allows for small intestinal sample collection after different reperfusion times in order to study the reperfusion phase continuously in a single patient. Since part of the small intestine is resected according to normal surgical procedures, no extra part of the small intestine is removed from the patient solely for study purposes, limiting patient risk. To study either the IR induced release of proteins from the isolated part of the small intestine or activation of plasma proteins, blood samples are collected at different time points from the efferent vessels of the isolated jejunum and arterial line, mostly present in left or right radial artery. This offers the possibility to study both systemic and local effects of the IR in either plasma or serum. Time points to collect plasma samples were: before the start of the operation after induction of anesthesia, before induction of ischemia, immediately upon reperfusion and every 30 minutes into the reperfusion period. Critical in this model is the availability of patients that undergo major abdominal surgery in which a part of the small intestine is resected. Additionally, a skilled surgical department embedded in a hospital setting equipped to execute these or comparable surgical procedures is needed to set up this human in vivo IR model of the small intestine. 


\section{MATERIAls}

\section{Reagents}

- $\quad$ Isopentane (2-methylbutane; Across Organics). ! CAUTION Isopentane is extremely flammable. Do not allow material to reach room temperature. Therefore, transport it in a plastic cup in a dry ice container.

- Liquid nitrogen ! CAUTION Liquid nitrogen is extremely cold $\left(-196^{\circ} \mathrm{C}\right)$ and will cause skin burn if in contact for more than $1 \mathrm{~s}$. Take precaution and be sure to protect eyes, fingers and exposed areas of skin.

- $\quad$ Dry ice ! CAUTION Dry Ice temperature is extremely cold $\left(-78.5^{\circ} \mathrm{C}\right)$. Handle dry ice with care and wear protective clothing and/ or appropriate gloves whenever touching it.

- Ice (water sludge further called ice)

- $\quad 3.7 \%-4.0 \%$ formalin fixation (Unifix, Klinipath) ! CAUTION Irritant; wear protective gloves.

- Glutaraldehyde ! CAUTION Severe skin and eye irritant; toxic by ingestion.

\section{Equipment}

Sterile equipment needed during surgery for study purposes

- Ultracision Harmonic Ace (Johnson\&Johnson, cat. no. ACE23P)

- $\quad$ Linear cutting stapler (GIA 6038S, Covidien)

- $\quad$ Staple cartridges (GIA 6038L, Covidien)

- $\quad$ Prolene 5-0 and PDS 3-0 Sutures (Johnson\&Johnson)

- $\quad$ Sterile atraumatic vascular clamps (Bulldog, Aesculap, cat. no. BH013R)

Equipment used by researchers

- $\quad$ Mouth masks (3M)

- $5 \mathrm{ml}$ EDTA vacuum tube (BD vacutainer, Becton Dickinson Diagnostics)

- 5 and 10 cc syringes (Becton Dickinson Diagnostics)

- $12 \mathrm{~cm}$ extension set (Steritex E98-3W, Codan)

- 25 G needle (BD Microlance 3, Becton Dickinson Diagnostics)

- $\quad$ Tissue Tek (Sakura Finetek)

- Cork sheets

- $\quad$ Sterile examination gloves

- $\quad$ Forceps (anatomical: Aesculap, cat. no. BD216R; surgical: Aesculap, cat. no. BD501R

- $\quad$ Scissors (Aesculap, cat. no. BC304R)

- $2 \mathrm{ml}$ sterilized tubes (Eppendorf)

- $3 \mathrm{ml}$ aluminium Cryo-tubes (Sanbio) 


\section{Chapter 5}

- $\quad$ Permanent marker

- Biopsy processing/paraffin embedding cassettes (Histosette II, Simport)

- 125 cc urine cup with screw cap (Böttger) to store formalin and incubate cassettes with tissue

- Pencil

- Cellulose cover

- I-FABP ELISA (Hycult Biotechnology)

\section{Reagent setup}

$--$

\section{Equipment setup}

- $\quad$ Before entering the operating theatre, always make sure to wear a mouth mask and keep sterile what has to be kept sterile around the operated patient.

- It is preferred to sample blood from the mesenteric venule with 2 people in a controlled manner, using a syringe which is connected to the needle via a $12 \mathrm{~cm}$ extension set.

\section{Procedure}

1) Positioning, anaesthesia of the patient (including insertion of an arterial line) and incision of the skin according to institutional routines.

CRITICAL STEP An arterial line is needed to sample systemic blood, which is used to measure arteriovenous concentration differences across the studied jejunum in order to explore local release and uptake of proteins.

2) After induction of anesthesia, before surgery the first 3cc blood sample is taken from the arterial line (mostly radial artery) using a 5cc syringe. Blood is transferred to a pre-chilled EDTA vacuum tube and kept on ice. All blood is at the end of the study centrifuged at $4000 \mathrm{rpm}, 4^{\circ} \mathrm{C}$ for 15 minutes to obtain plasma. Plasma is kept on ice and immediately stored in aliquots at $-80^{\circ} \mathrm{C}$ until analysis.

CRITICAL STEP Make sure that all EDTA tubes are labelled with the appropriate codes.

3) First part of surgery (opening of the abdominal wall, installation of a large self-retaining retractor) is according to standard procedures.

4) The peritoneal surface, the liver, the area around the pancreas, the hepatoduoneal ligament and the lymph nodes around the celiac axis are 
explored for metastases and localization of the major anatomical structures and tumor.

5) From this moment the $6 \mathrm{~cm}$ part of jejunum which can be studied has to be identified (Figure 1).

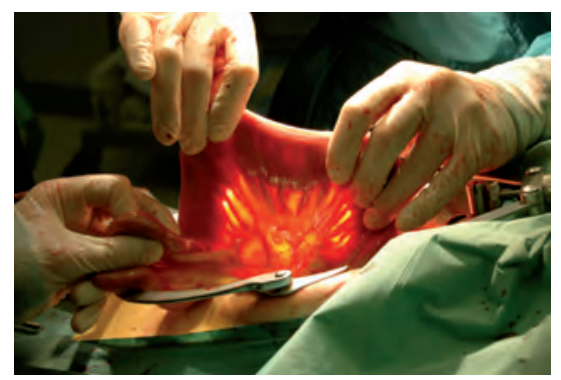

Figure 1: Identification of jejunum which can be isolated for study purposes. Note the clearly visible blood supply and drainage.

CRITICAL STEP Blood supply and drainage of the studied jejunum has to be clearly visible and to consist of 1 central mesenteric arteriole and venule.

6) To prevent blood flow from adjacent parts of the studied jejenunum, all collateral vessels are dissected and clamped and cut. Therefore, the mesentery is opened using Ultracision Harmonic Ace at the proximal and distal part of the suitable part of jejunum from the central mesenteric arteriole and venule until the serosa of the jejunum.

7) Isolation of the studied jejunum is performed by transsection at both ends with a linear cutting stapler (Figure 2).
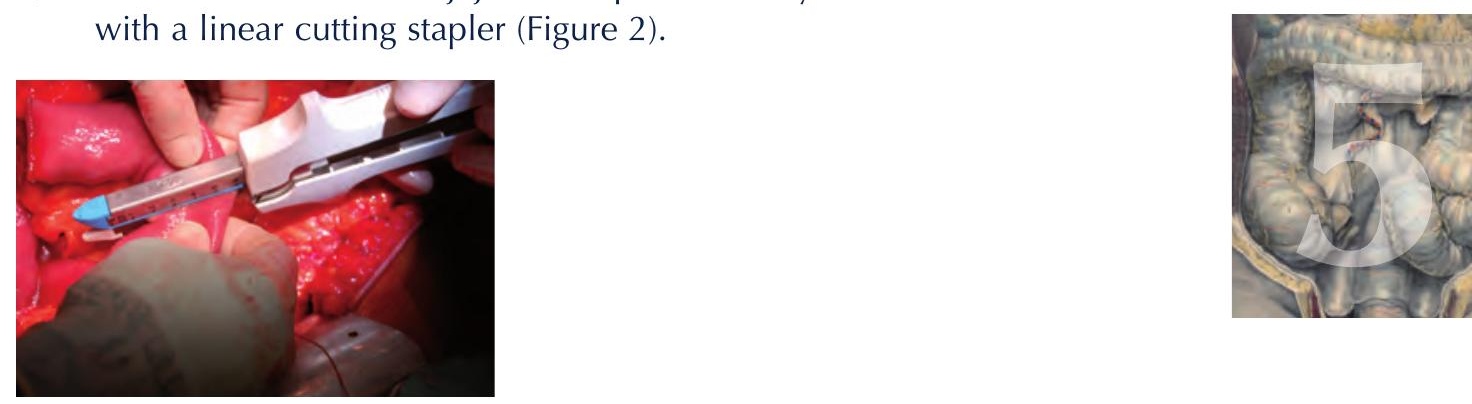

Figure 2: Isolation of the studied jejunum by a linear cutting stapler. 


\section{Chapter 5}

8) Both ends of the isolated jejunum are fixed with a single PDS 3-0 suture to the rest of the jejunum to prevent rotation.

CRITICAL STEP Leaving the isolated jejunum in the abdominal cavity without re-attaching to the proximal and distal parts of the jejunum may cause rotation around its axis, which causes disruption of the blood flow and thus re-introducing uncontrolled ischemia.

CRITICAL STEP The central mesenteric venule draining the isolated jejunum is dissected from its mesenteric surrounding to improve its accessibility for blood collection.

9) A 5 cc syringe, a $12 \mathrm{~cm}$ extension set and a $25 \mathrm{G}$ needle are given under sterile conditions to the scrub nurse.

10) The 5 cc syringe is connected via the extension set to the $25 \mathrm{G}$ needle by the nurse.

11) One surgeon takes the isolated jejunum with the left hand and inserts the needle into the venule, draining the isolated jejunum, while the other surgeon draws 3 cc blood using the syringe.

CRITICAL STEP It is difficult to draw blood from the venule by a single surgeon, because holding the jejunum, inserting the needle and drawing of blood may become a rather uncontrolled procedure, which may cause the needle to perforate the venule.

12) Hold a Prolene 5-0 suture with a small needle holder in order to repair the vessel with sutures if there is continued bleeding after removal of the needle.

13) The syringe is handed over to the researcher.

14) At the same time blood is drawn from the arterial line, as described in point 2.

15) Blood is processed and stored as described in point 2.

16) The isolated jejunum is subjected to 30 minutes ischemia with 2 atraumatic vascular clamps which are placed from both sides over the mesentery (Figure 3).
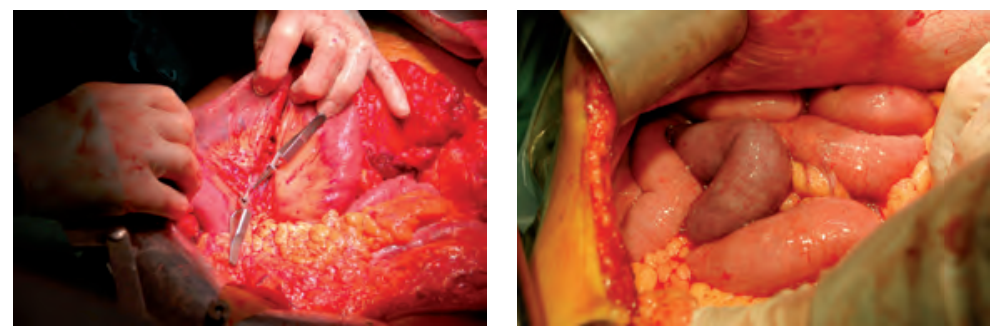

Figure 3: Clamping of the isolated jejunum (a) rapidly leads to discoloration of the jejunal wall (b). 


\section{TROUBLESHOOTING}

17) The isolated ischemic jejunum is placed in the abdominal cavity to guarantee warm ischemia.

18) The patient's temperature is constantly monitored and kept at $37^{\circ} \mathrm{C}$.

19) The surgical procedure proceeds as planned.

20) After half an hour of ischemia, one third $(2 \mathrm{~cm})$ of the isolated ischemic jejunum is resected for research purposes using linear cutting stapler.

21) The mesentery, near the serosa of the distal $2 \mathrm{~cm}$ of isolated ischemic jejunum, is opened using electrocoagluation.

22) The distal $2 \mathrm{~cm}$ of the isolated ischemic jejunum are dissected using a linear stapler.

23) This distal ischemic jejunum is handed over to the researchers.

CAUTION Avoid manipulation of the intestine, because this may cause exogenous damage. Therefore always use forceps.

CAUTION This human material has to be processed by the researchers wearing gloves.

24) The staples at both ends of the tissue are cut off with clean scissors and the tissue is processed to obtain paraffin-embedded sections, frozen sections, electron microscopy and tissue samples for PCR analysis or protein-based applications. This is performed by the researchers and described from points 24-36. In the meantime the surgeon continues at point 43.

\section{Paraffin-embedded sections and H\&E staining}

25) Cut defined $0.5 \mathrm{~cm}$ thick ring-like fragments of the jejunum.

26) Immerse immediately in 3.7\%-4.0\% formalin fixation and incubate overnight at room temperature.

CAUTION Irritant; wear protective gloves.

27) After 18-24 hours of formalin fixation embed the fragments of the jejunum in paraffin.

PAUSE POINT: Samples can be stored embedded in paraffin until ready for cutting.

28) Prepare $4 \mu \mathrm{m}$ paraffin cross-sections.

29) Stain sections with hematoxylin and eosin using appropriate standard procedures. Sections can be used for immunohistochemistry according to specific research questions.

\section{Frozen sections}

30) Cut defined $0.5 \mathrm{~cm} \times 0.5 \mathrm{~cm}$ squared fragments of jejunum

31) Immerse the jejunum with the use of forceps gently in Tissue Tek for 1 second. 
32) Adhere the jejunum in Tissue Tek to a piece of cork by dipping the jejunum to $0.3 \mathrm{~cm} \times 0.3 \mathrm{~cm}$ cork.

CRITICAL STEP Cork is used under the mounted tissue to enable subsequent cutting and easy mounting on a cryotome.

33) The jejunum, embedded in Tissue Tek, immersed and mounted on cork, is frozen in pre-chilled isopentane on dry ice.

CAUTION Isopentane is extremely flammable.

34) For cryosectioning, fix the cork on a mounting disk with tissue freezing medium.

35) When the tissue freezing medium is frozen, prepare $4 \mu \mathrm{m}$ frozen crosssections.

PAUSE POINT: Slides can be stored at $-20^{\circ} \mathrm{C}$ until stained. Electron microscopy

36) Electron microscopy preparation solution is pre-chilled on ice.

CAUTION Glutaraldehyde is a severe skin and eye irritant and toxic by ingestion.

37) $24 \mathrm{~mm}^{3}$ blocks are collected from each tissue specimen and stored in glutaraldehyde.

38) The samples are kept cold $\left(4^{\circ} \mathrm{C}\right)$ in glutaraldehyde solution for at least 18 hours.

39) Further preparation according to local routines.

Tissue sampling for PCR analysis or protein-based applications (e.g. Western blot, ELISA)

40) Cut $0.5 \mathrm{~cm} \times 0.5 \mathrm{~cm}$ squared fragments of jejunum.

41) Snap-freeze fragments in liquid nitrogen and store until use at $-80^{\circ} \mathrm{C}$.

CAUTION Liquid nitrogen is extremely cold $\left(-196^{\circ} \mathrm{C}\right)$ and will cause skin burn if in contact for more than $1 \mathrm{~s}$.

PAUSE POINT: Samples can be stored at $-80^{\circ} \mathrm{C}$ until used.

42) While the researcher prepares the tissue, the surgeon removes the vascular clamps. The reperfusion is initiated.

43) After removal of the clamp, the isolated jejunum is inspected for restoration of blood flow.

TROUBLESHOOTING

44) Immediately thereafter, blood is collected from the mesenteric venule and from the arterial line, as described from point 9-15.

45) Isolated jejunum and arterial and venous blood can be collected at different time points, depending on the research questions.

CRITICAL STEP To study acute damage of the epithelial lining, it is 
recommended to take tissue and blood samples 15-30 minutes after start of reperfusion. To study repair of the epithelium, collect samples from 60 minutes after reperfusion onwards. See anticipated results.

46) The maximum time of reperfusion of the isolated ischemic jejunum is dependent on the surgical procedure, i.e. the moment at which the surgeon decides that the pancreatico- or hepaticojejunal anastomosis has to be created. In the proposed surgical procedures reperfusion time varies from 30 minutes to 4 hours.

47) At the time the last isolated reperfused jejunum is obtained for the study, also $2 \mathrm{~cm}$ of the proximal jejunum, which was not isolated and remained untreated during the surgery, is resected using a GIA. This tissue is used as internal control tissue: it is from the same patient and experienced similar surgical handling as the isolated jejunum, while it was not subjected to IR.

48) Surgery continues as anticipated.

Intestinal Fatty Acid Binding Protein (I-FABP) arteriovenous concentration differences across studied jejunum assesses epithelial cell damage

49) In arterial and venous plasma the concentrations of I-FABP are measured by means of the commercially available enzyme-linked immunosorbent assay (ELISA) used according to manufacture guidelines.

50) Subtract the venous from the arterial plasma levels of I-FABP, which are collected at the same point, in order to obtain arteriovenous concentration differences across the studied jejunum. 


\section{TROUBLESHOOTING}

Troubleshooting advice can be found in Table 1.

\begin{tabular}{|c|c|c|c|}
\hline Step & Problem & Possible reason & Solution \\
\hline 16 & $\begin{array}{l}\text { Isolated jejunum does not } \\
\text { become ischemic after } \\
\text { clamping }\end{array}$ & $\begin{array}{l}\text { 1) The proximal and/ or distal part of jejunum } \\
\text { is not completely stapled and dissected; } \\
\text { collaterals remain } \\
\text { 2) The mesentery is not completely } \\
\text { closed/ clamped; collaterals remain } \\
\text { 3) The vascular clamps are not strong enough }\end{array}$ & $\begin{array}{l}\text { Check whether the studied } \\
\text { jejunum is perfectly isolated } \\
\text { until the point where you } \\
\text { want to clamp } \\
\text { Use } 2 \text { stronger clamps }\end{array}$ \\
\hline$>43$ & $\begin{array}{l}\text { During reperfusion, the } \\
\text { isolated jejunum stays or } \\
\text { becomes ischemic }\end{array}$ & $\begin{array}{l}\text { 1) The isolated jejunum is rotated around } \\
\text { its axis } \\
\text { 2) The mesenteric venule is thrombotic } \\
\text { (due to blood drawing?) }\end{array}$ & $\begin{array}{l}\text { In both cases, reperfusion is not } \\
\text { complete and not useful to study } \\
\text { reperfusion. Dissect the studied } \\
\text { jejunum. Next time suture the iso- } \\
\text { lated jejunum to the rest of the } \\
\text { jejunum with one or two stitches } \\
\text { and after each drawing of blood } \\
\text { control the hemorrhage. }\end{array}$ \\
\hline
\end{tabular}

\section{ANTICIPATED RESULTS}

We describe a newly developed human experimental model to study intestinal IR induced cell damage and its direct consequences. Successful ischemia is observed almost directly after placement of the vascular clamp in the 29 patients studied so far by both discoloration of the small jejunum wall and termination of the peristalsis of the studied jejunum. Immediately upon reperfusion, the isolated jejunum regains its pinkish color and motility. Histologic analysis of hematoxylin/ eosin-stained sections shows consistent results ${ }^{12}$. The control jejunum, which neither is isolated, nor subjected to IR, shows normal architecture. Directly after ischemia the intestinal epithelial lining is microscopically normal, while subepithelial spaces appear at the villus tip. However, after 25 minutes reperfusion shedding of differentiated epithelial cells into the lumen is found with a compromised epithelial lining. Interestingly, within 60 minutes reperfusion the epithelial barrier reseals, while debris of apoptotic, shedded epithelial cells is observed in the lumen. In order to further objectivate the intestinal mucosal cell 
damage in this model, Intestinal Fatty Acid Binding Protein (I-FABP) arteriovenous concentration differences are measured in plasma using ELISA before and after ischemia. I-FABP is a small cytosolic protein constitutively present in mature enterocytes and released upon cell membrane integrity loss ${ }^{13-15}$. Mean (SEM) arteriovenous concentration gradients of I-FABP across studied jejunum in 20 patients reveal rapid epithelial cell damage. I-FABP release increased significantly from 328 (59) pg/ml before ischemia towards 4,096 (543) pg/ml immediately after ischemia $(\mathrm{p}<0.001)$ and declined gradually to 1,204 (408) pg/ml after 2 hours reperfusion $(p<0.001)^{12}$. In conclusion, this unique and harmless human IR model of the jejunum enables the study of acute damage to the epithelial lining and its subsequent repair mechanisms.

\section{References}

1. Oldenburg, W.A., Lau, L.L., Rodenberg, T.J., Edmonds, H.J. \& Burger, C.D. Acute mesenteric ischemia: a clinical review. Arch. Intern. Med. 164, 1054-1062 (2004).

2. Fukatsu, K. et al. Gut ischemia-reperfusion affects gut mucosal immunity: a possible mechanism for infectious complications after severe surgical insults. Crit. Care Med. 34, 182-187 (2006).

3. Mallick, I.H., Yang, W., Winslet, M.C. \& Seifalian, A.M. Ischemia-reperfusion injury of the intestine and protective strategies against injury. Dig. Dis. Sci. 49, 1359-1377 (2004).

4. Vicente, D.C. \& Kazmers, A. Acute mesenteric ischemia. Curr. Opin. Cardiol. 14, 453458 (1999).

5. Yasuhara, H. Acute mesenteric ischemia: the challenge of gastroenterology. Surg. Today 35, 185-195 (2005).

6. Fink, M.P. \& Delude, R.L. Epithelial barrier dysfunction: a unifying theme to explain the pathogenesis of multiple organ dysfunction at the cellular level. Crit. Care Clin. 21, 177-196 (2005).

7. Chiu, C.J., McArdle, A.H., Brown, R., Scott, H.J. \& Gurd, F.N. Intestinal mucosal lesion in low-flow states. I. A morphological, hemodynamic, and metabolic reappraisal. Arch. Surg. 101, 478-483 (1970).

8. Carden, D.L. \& Granger, D.N. Pathophysiology of ischaemia-reperfusion injury. J.

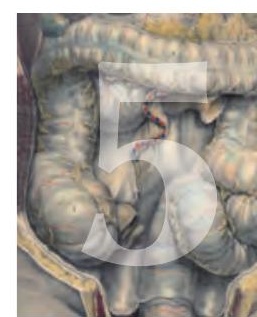
Pathol. 190, 255-266 (2000).

9. Chen, L.W. et al. The two faces of IKK and NF-kappaB inhibition: prevention of systemic inflammation but increased local injury following intestinal ischemiareperfusion. Nat. Med. 9, 575-581 (2003).

10. Robinson, J.W., Mirkovitch, V., Winistorfer, B. \& Saegesser, F. Response of the intestinal mucosa to ischaemia. Gut 22, 512-527 (1981). 


\section{Chapter 5}

11. Blikslager, A.T., Moeser, A.J., Gookin, J.L., Jones, S.L. \& Odle, J. Restoration of barrier function in injured intestinal mucosa. Physiol. Rev. 87, 545-564 (2007).

12. Derikx, J.P. et al. Rapid reversal of human intestinal ischemia-reperfusion induced damage by shedding of injured enterocytes and reepithelialisation. PLOS ONE 3, e3428 (2008).

13. Kanda, T. et al. Intestinal fatty acid-binding protein is a useful diagnostic marker for mesenteric infarction in humans. Gastroenterology 110, 339-343 (1996).

14. Lieberman, J.M., Sacchettini, J., Marks, C. \& Marks, W.H. Human intestinal fatty acid binding protein: report of an assay with studies in normal volunteers and intestinal ischemia. Surgery 121, 335-342 (1997).

15. Derikx, J.P. et al. Urine based detection of intestinal mucosal cell damage in neonates with suspected necrotising enterocolitis. Gut 56, 1473-1475 (2007). 


\subsection{Rapid reversal of human intestinal ischemia-reperfusion induced damage by shedding of injured enterocytes and reepithelialisation}

Joep P. M. Derikx, Robert A. Matthijsen, Adriaan P. de Bruïne,

Annemarie A. van Bijnen, Erik Heineman, Ronald M. van Dam, Cornelis H. C. Dejong, Wim A. Buurman PLOS ONE (2008) 3: e3428. 


\title{
Chapter 5
}

\begin{abstract}
Background

Intestinal ischemia-reperfusion (IR) is a phenomenon related to physiological conditions (e.g. exercise, stress) and to pathophysiological events (e.g. acute mesenteric ischemia, aortic surgery). Although intestinal IR has been studied extensively in animals, results remain inconclusive and data on human intestinal IR are scarce. Therefore, an experimental harmless model for human intestinal IR was developed, enabling us to clarify the sequelae of human intestinal IR for the first time.
\end{abstract}

\section{Methods and findings}

In 30 patients undergoing pancreatico-duodenectomy we took advantage of the fact that in this procedure a variable length of jejunum is removed. Isolated jejunum $(5 \mathrm{~cm})$ was subjected to 30 minutes ischemia followed by reperfusion. Intestinal Fatty Acid Binding Protein (I-FABP) arteriovenous concentration differences across the bowel segment were measured before and after ischemia to assess epithelial cell damage. Tissue sections were collected after ischemia and at 25, 60 and 120 minutes reperfusion and stained with $\mathrm{H} \& \mathrm{E}$, and for I-FABP and the apoptosis marker M30. Bonferroni's test was used to compare I-FABP differences.

Mean (SEM) arteriovenous concentration gradients of I-FABP across the jejunum revealed rapidly developing epithelial cell damage. I-FABP release significantly increased from $290(46) \mathrm{pg} / \mathrm{ml}$ before ischemia towards 3,997 (554) $\mathrm{pg} / \mathrm{ml}$ immediately after ischemia $(\mathrm{p}<0.001)$ and declined gradually to $1,143(237) \mathrm{pg} / \mathrm{ml}$ within 1 hour reperfusion $(\mathrm{p}<0.001)$. Directly after ischemia the intestinal epithelial lining was microscopically normal, while subepithelial spaces appeared at the villus tip. However, after 25 minutes reperfusion, enterocyte M30 immunostaining was observed at the villus tip accompanied by shedding of mature enterocytes into the lumen and loss of I-FABP staining. Interestingly, within 60 minutes reperfusion the epithelial barrier resealed, while debris of apoptotic, shedded epithelial cells was observed in the lumen. At the same time, M30 immunoreactivity was absent in intact epithelial lining.

\section{Conclusions}

This is the first human study to clarify intestinal IR induced cell damage and repair and its direct consequences. It reveals a unique, endogenous clearing mechanism for injured enterocytes: rapid detachment of damaged apoptotic enterocytes into the lumen. This process is followed by repair of the epithelial continuity within an hour, resulting in a normal epithelial lining. 


\section{INTRODUCTION}

Intestinal ischemia-reperfusion (IR) injury is an important (patho)physiological mechanism, potentially leading to a compromised mucosal barrier in numerous situations 1,2 . Intestinal hypoperfusion occurs during exercise both in healthy relatively untrained volunteers and in highly trained athletes, which stresses the abdominal vasodilator reserve by diverting blood flow from the splanchnic circulation to the exercise muscles ${ }^{3-5}$. This substantial reduction in blood flow is sometimes associated with symptoms of abdominal hypoperfusion, which are almost always reversible without intensive therapy ${ }^{3-5}$. Stress may also lead to symptomatic intestinal ischemia via increased catecholamine release, resulting in severe splanchnic vasospasm ${ }^{6}$. Furthermore, intestinal IR may be looked at as a common pathway in several pathologies, where temporary reduction in flow may result from inherent vascular disease (thrombosis, shock, embolism, vasculitis) or from inflammatory diseases affecting perfusion. An example of inherent vascular disease is acute mesenteric ischemia (AMI), which carries a high morbidity and mortality rate, both increased by frequent delays in diagnosis. Such diagnostic delays are mainly caused by non-specific signs and symptoms and limited diagnostic accuracy of the laboratory and radiological tests currently in use. Arterial and venous thromboembolisms are the main causes of $\mathrm{AMI}{ }^{1,2}$. More global hypoperfusion may result from shock. Along these lines, intestinal IR is considered to be a crucial phenomenon involved in the onset of necrotizing enterocolitis, in the pathogenesis of small intestinal transplantation and rejection, and in the potential development of postoperative or posttraumatic complications, such as systemic inflammatory response syndrome, sepsis and multiple organ failure $2,7,8$. Here, inflammation and collateral damage may play a role.

Generally, IR injury starts with affecting cell metabolism, ultimately resulting in cell death (apoptosis as well as necrosis) ${ }^{9-11}$. Upon reperfusion, ischemia primed cells are prone to generate toxic reactive oxygen metabolites and release of constitutive cellular proteins upon loss of cell membrane integrity, which can act as danger signals and activate the immune system ${ }^{9-15}$. In the resulting inflammatory response, local expression of adhesion molecules, proinflammatory mediators and activation of neutrophils as well as the complement system are central mediators of IR induced tissue injury ${ }^{10,11,13-16}$. This proinflammatory state increases tissue vulnerability to further injury, resulting in the amplification and derailment of the inflammatory response, although it is meant to be a beneficial reaction to clear damaged cells $11,14,16$.

The pathophysiology of intestinal IR has hitherto mainly been studied in 


\section{Chapter 5}

animal models (rodents, cats, dogs and pigs) by transient clamping of the superior mesenteric artery ${ }^{17-19}$. First event after intestinal IR in all these animals is the appearance of subepithelial spaces at the villus tips, immediately followed by loss of the highly susceptible mature enterocytes ${ }^{17,}{ }^{18}$. Depending on the duration of ischemia, this sloughing may continue toward the crypt, causing denuded villi ${ }^{17,18}$. In line with general IR-induced cell damage, also in intestinal IR, apoptosis is the major mode of cell death in the destruction of epithelial cells ${ }^{20}$. Moreover, the disruption of the interaction between epithelial cells and extracellular matrix ('anoikis') may play an important role in the onset of apoptosis in detached enterocytes ${ }^{20}$. Some studies report that after longer periods of reperfusion, migration of epithelial cells to seal the exposed basement membrane occurs, which closes the loss of epithelial continuity ${ }^{19,21}$. Further studies show that reperfusion leads to inflammation, resulting in transmural infarction ${ }^{19,22-24}$. This inflammatory response is comparable to the response of other organs to IR induced cell damage, including infiltration of neutrophils and activation of complement ${ }^{22,24}$.

The histological consequences of human gut IR are known from patients operated upon for different gastrointestinal disorders after obvious signs of clinical shock or from post-mortem studies ${ }^{25}$. Microscopic examination of gut segments of these patients all showed characteristic mucosal lesions, ranging from epithelial lifting at the villus tip to denuded villi with disintegrated and hemorrhagic lamina propria ${ }^{25}$. The histological findings concerned end-stages of diseases and were identical to the previously described ultimate phases of intestinal mucosal IR-induced damage in animals.

This study was directed at revealing the sequelae of intestinal IR in man in the course of time, using a newly developed human experimental, controlled model of 30 minutes intestinal ischemia, followed by variable periods of reperfusion.

\section{Methods}

\section{Patients and experimental, surgical procedure}

Thirty patients (12F:18M) with a median age of 68.5 years (range: $35-79$ years) undergoing pancreatic surgery were studied. Twenty-eight of these patients underwent a standard pylorus-preserving or classical pancreatico-duodenectomy (Whipple's procedure) for benign or malignant disease, whilst two patients underwent a Frey procedure for chronic pancreatitis. During pancreaticoduodenectomy a variable length of jejunum is usually resected in continuity with the specimen. Similarly, in creating a Roux-limb for the pancreatico- 
jejunostomy in Frey's procedure, it is often necessary to resect a small segment of jejunum that is less well perfused. We took advantage of this, which enabled us to study IR induced cell damage in a harmless human jejunal IR model. To that purpose, the most distal part of the jejunum to be resected with the Whipple specimen, or the most proximal part of the Roux-limb to be used as a pancreatico-jejunostomy in the Frey procedure, was used to isolate a $6 \mathrm{~cm}$ segment of jejunum. The isolated jejunum $(6 \mathrm{~cm})$ was subjected to 30 minutes ischemia followed by a median reperfusion time of 128 minutes (range: 0-210 minutes) according to the following experimental protocol.

In short, the first part of surgery (opening of the abdominal wall, installation of a large self-retaining retractor and exploration for metastases and localization of the major anatomical structures and tumour) was according to standard procedures. From this moment the $6 \mathrm{~cm}$ part of jejunum, which was going to be studied was identified and care was taken that the vasculature of the studied jejunum consisted of 1 central mesenteric arteriole and venule. This was achieved by dissection, clamping and cutting of all collateral mesenterial vessels to the studied jejunum using Ultracision Harmonic Ace (Johnson\&Johnson, cat. no. ACE23P, Amersfoort, the Netherlands). Thereafter, the segment of jejunum was further isolated by transsection at both ends with a linear cutting stapler (GIA 6038S, Covidien, Zaltbommel, the Netherlands). The isolated jejunum was then subjected to 30 minutes ischemia with 2 atraumatic vascular clamps, which are placed over the mesentery (Bulldog, Aesculap, cat. no. BH013R, Tuttlingen, Germany). The isolated ischemic jejunum was subsequently placed in the abdominal cavity to guarantee warm ischemia. The surgical procedure proceeded as planned. After half an hour of ischemia, one third $(2 \mathrm{~cm})$ of the isolated ischemic jejunum was resected using linear cutting stapler to study early phenomena during ischemia. Reperfusion was initiated by removal of the clamps, and the isolated jejunum was inspected for restoration of blood flow. A further segment of isolated jejunum $(2 \mathrm{~cm})$ was resected similarly after 25 minutes of reperfusion to study early phenomena during reperfusion. The last part of studied jejunum was resected from 60 minutes after reperfusion onwards to investigate late phenomena during reperfusion. At the time the last isolated reperfused jejunum was obtained for the study, also $2 \mathrm{~cm}$ of jejunum, which had not been isolated and remained untreated during the surgery, was resected using a linear cutting stapler. This tissue was used as internal control tissue: it was from the same patient and experienced similar surgical handling as the isolated jejunum, while it was not subjected to IR. Then, our study-protocol was ended and surgery continued as anticipated. 


\section{Chapter 5}

\section{Histological assessment}

The tissue specimens obtained at four different time points during the study (after half an hour of ischemia, after 25 minutes of reperfusion, from 60 minutes reperfusion onwards and internal control) were immediately immersed in $3.7 \%-4.0 \%$ formaldehyde fixation (Unifix, Klinipath, Duiven, the Netherlands) and incubated overnight at room temperature. Next, the formalin fixed samples were embedded in paraffin and $4 \mu \mathrm{m}$ sections were cut. Sections were stained with haematoxylin and eosin (H\&E) using standard histological techniques.

During the conduct of the experiments, it was noted that in every jejunal sample we collected at the time of maximal reperfusion, a milky substance appeared from the lumen. In order to study the content of this substance, we collected and fixed it according to the AgarCyto cell block procedure ${ }^{26}$. Shortly, the collected cells were fixed in Unifix (Klinipath) for 24 hours. Fixed cells were centrifuged for 5 minutes at $1200 \mathrm{rpm}$. The pellet was carefully resuspended in $1 \mathrm{ml} 2 \%$ liquid agarose at $65^{\circ} \mathrm{C}$ (LE, analytical grade; Promega, Madison, WI) and transferred into a 1.5-ml Eppendorf tube. The tube was centrifuged for 5 minutes at $1000 \mathrm{rpm}$ to concentrate the cells in the agar. The agar-cell pellet was solidified at $4^{\circ} \mathrm{C}$ for at least 1 hour. The agar cone was carefully taken out of the reaction tube with pointed forceps and divided in two halves in the sagittal plane of the cone. The two agar pieces were embedded in paraffin under standard conditions for surgical biopsies. This paraffin embedded agar-cell pellet is called AgarCyto ${ }^{26}$. From the AgarCyto, $4 \mu \mathrm{m}$ sections were cut. For cytomorphologic examination, AgarCyto sections were stained with $\mathrm{H} \& \mathrm{E}$.

\section{Immunohistochemistry}

Paraffin sections were dewaxed in xylene and rehydrated in graded ethanol to distilled water. Endogenous peroxidase activity was blocked using $0.3 \%$ hydrogen peroxide in methanol for 12 minutes. Sections undergoing heatmediated antigen retrieval were placed in a cooker filled with $10 \mathrm{mM}$ citrate buffer ( $\mathrm{pH}$ 6.0) for 10 minutes for staining with collagen IV. After blocking the non-specific antibody binding using 5\% BSA, the sections were incubated with specific primary antibody at room temperature for 45 minutes. The following primary antibodies were used: mouse anti-cleaved cytokeratin 18 antibody, clone M30 (Catalogue no. 10700; Peviva, Bromma, Sweden); mouse antiCollagen IV, clone CIV 22 (Catalogue no. M0785; DAKO, Glostrup, Denmark); mouse anti-smooth muscle actin (SMA), clone 1A4 (Catalogue no. M0851; DAKO); and chicken anti-intestinal-fatty acid binding protein (I-FABP, kindly provided by Hycult Biotechnology (HBT; Uden, the Netherlands)). After 
washing, an appropriate biotin-conjugated secondary antibody was used. Binding of the primary antibody was demonstrated by the streptavidin-biotin system (DAKO) and visualized by applying 3-amino-9-ethylcarbazole (AEC; Sigma, St. Louis, MO). For I-FABP, peroxidase-conjugated rabbit anti-chicken IgY (Catalogue no. 303-035-003; Jackson ImmunoResearch Europe, Suffolk, UK) was used as secondary antibody and AEC was used as chromogen. For staining of collagen IV, labelled polymer detection agent (PowerVision PolyHRP-anti-Mouse/Rabbit/Rat IgG (Immunologic; Duiven, The Netherlands)) was applied followed by AEC. Nuclei were counterstained with haematoxylin. The stained slides were photographed by a Nikon eclipse E800 microscope with a Nikon digital camera DXM1200F. No significant staining was detected in slides incubated with control rabbit serum and/or mouse IgG instead of the primary antibody indicating the absence of significant background staining.

\section{Immunofluorescence}

Cryostat sections $(4 \mu \mathrm{m})$ were cut and stained for Zona Occludens-1 (ZO-1). Briefly, slides were dried, fixed in $4 \%$ paraformaldehyde for 15 minutes and non-specific antibody binding was blocked using $10 \%$ normal goat serum. Next, slides were stained with anti ZO-1 (catalogue no. 61-7300; Zymed Laboratories Inc., San Francisco, CA). Slides were incubated with a Texas Red labelled secondary antibody (Jackson, West-Grove, PA). Subsequently, slides were mounted using glycerol-PBS with 1, 4-diazabicyclo $(2,2,2)$ octane (DABCO) and 4, 6-diamidinol (2)-phenylindole (DAPI), and viewed with an immunofluorescence microscope.

\section{Blood sampling and I-FABP measurement}

Arterial blood was sampled preoperatively, before the isolated jejunum was subjected to ischemia, immediately upon reperfusion and every half hour during reperfusion until the end of the study protocol. Simultaneous with the second (i.e. before ischemia) and every next arterial blood sample, blood was drawn from the venule draining the isolated jejunal segment by direct puncture to assess concentration gradients across the isolated jejunal segment. All blood samples were directly transferred to pre-chilled EDTA vacuum tubes (BD vacutainer, Becton Dickinson Diagnostics, Aalst, Belgium) and kept on ice. At the end of the study all blood samples were centrifuged at $4000 \mathrm{rpm}, 4^{\circ} \mathrm{C}$ for 15 minutes to obtain plasma. Plasma was kept on ice and immediately stored in aliquots at $-80^{\circ} \mathrm{C}$ until analysis.

Intestinal Fatty Acid Binding Protein (I-FABP) concentrations were measured in plasma by means of the commercially available enzyme-linked 


\section{Chapter 5}

immunosorbent assay (ELISA) used according to manufacture guidelines and kindly provided by HBT. I-FABP arteriovenous concentration differences were calculated (mesenteric venule minus radial artery) before and after ischemia to assess epithelial cell damage.

\section{Ethics}

The study was approved by the Medical Ethics Committee of the Maastricht University Medical Centre + and all individuals gave written informed consent.

\section{Statistics}

Statistical analysis was performed using Prism 4.0 for Windows (GraphPad Software Inc. San Diego, CA). I-FABP arteriovenous concentration gradients were presented as mean \pm standard error (SEM). Normality of all data obtained was verified by Kolmogorov-Smirnov test. Bonferroni's multiple comparison test was used (after significant repeated measures ANOVA) to compare I-FABP arteriovenous concentration differences in time. A p-value below 0.05 was considered to be statistically significant.

\section{RESULTS}

\section{Early phenomena during ischemia}

At the end of the ischemic period of 30 minutes, H\&E sections of the jejunum showed microscopically normal epithelial lining (Figure 1A, B). However, subepithelial spaces appeared at the villus tip. To study the epithelial cells in more detail, immunohistochemical staining of I-FABP was performed, a small protein present in the cytoplasma of differentiated enterocytes [27]. Further, we stained ZO-1, a $225 \mathrm{kDa}$ membrane bound protein, binding the transmembrane tight junction proteins occludin and claudins and linking them to cytoskeletal actin [28]. Intensive immunostaining for I-FABP was observed in the control jejunal epithelium, mainly in the cytoplasm of the mature enterocytes and goblet cells in the upper half of the villi, whereas cells in the crypts were not stained, as previously observed in experiments by our group (unpublished results) (Figure 2A). A decreased staining of I-FABP was observed in jejunal mature epithelial cells, after 30 minutes ischemia, while intense staining was found in the subepithelial spaces (Figure 2B), indicating early leakage of I-FABP from the cytoplasm of intestinal epithelial cells into the subepithelial space. ZO-1 was detected at the apical pole of the epithelial cells in both the control jejunum and the jejunum after an ischemic period of 30 minutes (data not shown).

To clarify the development of the subepithelial spaces, the basement 
membrane, demarcating the lamina propria from the epithelial cells, and the underlying network of myofibroblasts within the villus lamina propria were studied, by staining collagen IV and smooth muscle actin (SMA), respectively $[29,30]$. A clear retraction of the basement membrane was found from the basal pole of the epithelial cells at the tip of the villi (Figure 3A, B). In line with this, shorter myofibroblasts were observed in a denser lamina propria (data not shown).
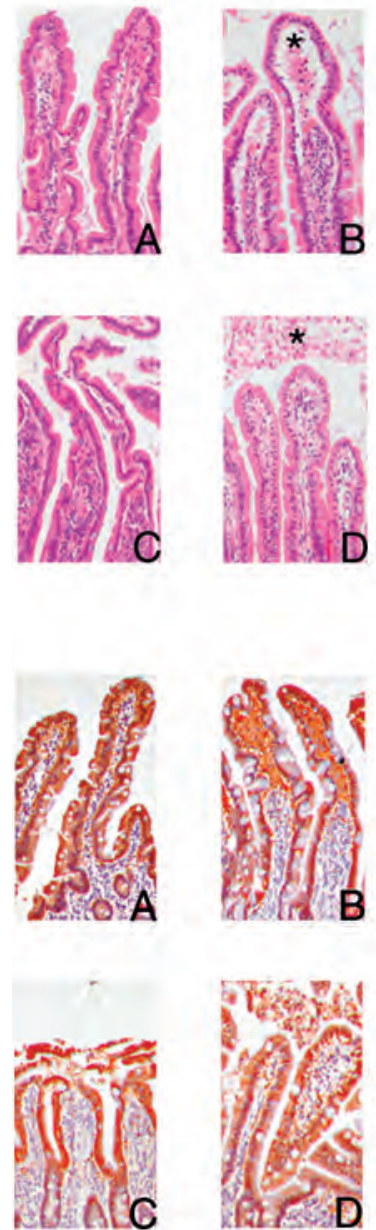

Figure 1: Histological analysis of H\&E-stained jejunal sections (100x) shows a normal epithelial lining in untreated tissue (A), and upon 30 minutes of ischemia (B). However, subepithelial spaces $(*)$ appear after 30 minutes ischemia (B). After 25 minutes reperfusion shedding of mature enterocytes into the lumen is found (C). Within 60 minutes of reperfusion the epithelial lining is resealed, while debris of apoptotic, shedded enterocytes $(*)$ are found in the lumen (D).

Figure 2: Immunolocalization of I-FABP in red (3-amino-9ethylcarbazole, AEC) (100x) in the control jejunum not subjected to ischemia-reperfusion (A) shows an abundant cytosolic presence of I-FABP in the epithelial cells of the upper half of the villus. Upon 30 minutes ischemia (B), cytosolic I-FABP staining is decreased in mature enterocytes with abundant staining in the subepithelial spaces. A decreased cytosolic staining is still observed after 25 minutes reperfusion $(C)$. Within 60 minutes reperfusion, IFABP cytosolic positive cells are part of the resealed epithelial barrier (D), while shedded I-FABP containing enterocytes are found in the debris in the lumen.

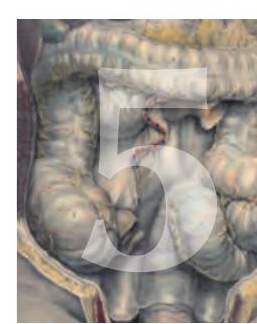



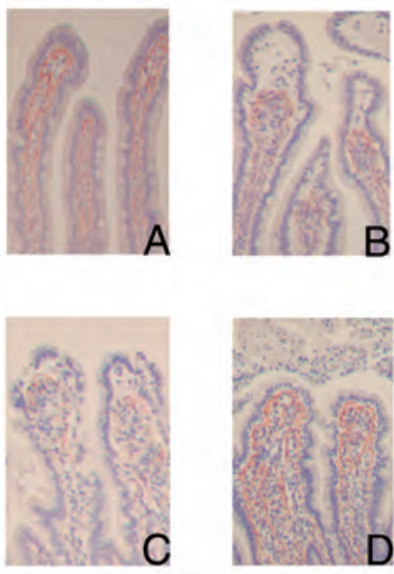

Figure 3: Characterization of the basement membrane with collagen IV staining in red (AEC) shows collagen IV positive cells directly beneath the epithelial layer in control jejunum (100x) (A). Upon 30 minutes of ischemia, a clear retraction is found of the collagen IV positive cells from the basal pole of the epithelial cells at the tip of the villus, causing subepithelial spaces (B). After 25 minutes reperfusion, the retracted basement membrane is still observed (C). Within 60 minutes reperfusion, the collagen IV positive basement membrane is again attached to the epithelial lining (D).

\section{Early phenomena during reperfusion}

To investigate loss of enterocyte membrane integrity, arteriovenous concentration differences of I-FABP across the studied jejunum were measured before ischemia and from reperfusion onwards. All individuals showed a basal release of mean (SEM) I-FABP of $290(46) \mathrm{pg} / \mathrm{ml}$ from the isolated jejunal segment before ischemia, potentially reflecting the physiologic turnover of enterocytes (Figure 4). Interestingly, I-FABP release significantly increased towards 3,997 (554) pg/ml immediately after ischemia and upon reperfusion $(p<0.001)$, suggesting rapid epithelial cell damage following 30 minutes ischemia. A gradual, significant decline to 1,143 (237) $\mathrm{pg} / \mathrm{ml}$ of I-FABP release occurred within 1 hour reperfusion ( $p<0.001$, immediately upon ischemia vs. 1 hour reperfusion).

After 25 minutes of reperfusion, structural damage to the mucosa became apparent, consisting of detachment of epithelial cells, particularly in the apical regions of the villi, leading to shedding of (sheets of) mature epithelial cells into the lumen (Figure 1C). Intestinal villus epithelial cells, which had lost the contact with the underlying villous stroma, underwent a phenomenon called 'anoikis', which refers to detachment-induced apoptosis [31]. Therefore, apoptosis was visualized in the jejunal sections using the apoptosis marker M30, which detects the Asp396 caspase cleavage site in cytokeratin-18 [32]. Intense M30 immunostaining was observed after 25 minutes of reperfusion at the villus tips, at the site where extensive desquamation of the epithelial cells from the villus tips into the intestinal lumen occurred, which was not detected in the earlier phase (Figure 5A-C). In the shortly reperfused jejunum a substantial loss of ZO1 expression was shown in the sheets of shedded cells, which was accompanied by a decreased cytoplasmatic immunoreactivity of I-FABP. 


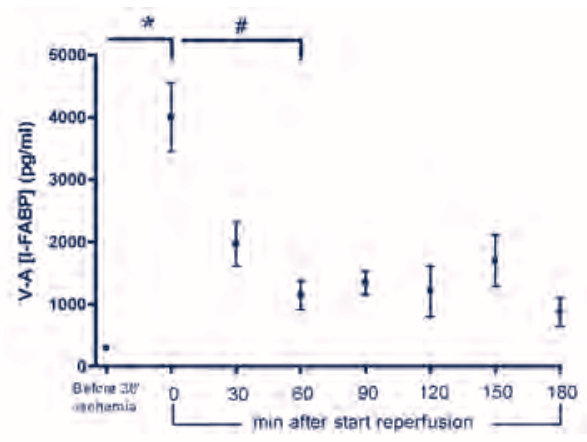

Figure 4: Arteriovenous concentration differences of I-FABP (mesenteric venule minus radial artery), a marker for acute enterocyte damage, across the isolated jejunal segment show rapid significant release of I-FABP after ischemia, while concentration differences of I-FABP gradually reduce following reperfusion. ${ }^{*} p<0.001, \# p<0.001$
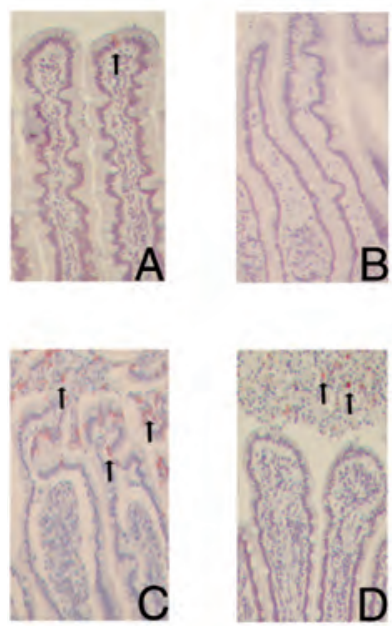

Figure 5: Apoptosis, assessed by M30 staining in red (AEC). An M30 positive cell (arrow) is observed at the top of the villus in normal jejunum (100x) (A). Upon 30 minutes ischemia, no M30 positive staining is found (B), while after 25 minutes reperfusion intense M30 immunostaining is observed at the villus tip near the shedding of mature epithelial cells into the lumen (arrows) (C). At 60 minutes reperfusion M30 immunoreactivity is no longer detectable in the intact, resealed epithelial barrier, while debris of M30 positive, shedded epithelial cells is observed in the lumen (D). 


\section{Chapter 5}

\section{Late phenomena during reperfusion}

As mentioned in the Materials and Methods section, a notable finding in all jejunal samples collected after 30 minutes ischemia and more than 60 minutes reperfusion was the presence of a milky substance that appeared from the lumen. Histological analysis of this debris revealed sheets of enterocytes with numerous apoptotic cells, assessed by H\&E and M30 staining (Figure 6A, B).

The H\&E staining of the jejunum, which underwent 30 minutes ischemia and more than 60 minutes reperfusion, confirmed the debris of detached epithelial cells in the lumen (Figure 1D). Interestingly, within 60 minutes reperfusion the epithelial layer resealed and full confluent coverage of the villus tip by enterocytes was observed again, indicating a recovered epithelial barrier (Figure 1D). The cells coating the lamina propria were immunopositive for IFABP and ZO-1, indicating that the epithelial continuity contained viable enterocytes with intact tight junctions (Figure 2D). Immunostaining with collagen IV showed that the basement membrane was again attached to the epithelial lining (Figure 3D). To investigate the status of these epithelial cells, M30 immunostaining was performed to assess apoptosis. M30 immunoreactivity was absent in the newly formed intact epithelium, while the debris of shedded epithelial cells in the lumen contained apoptotic cells (Figure 5D).
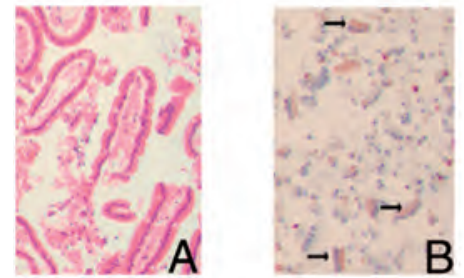

Figure 6: Histological analysis of H\&E stained AgarCyto-fixed debris, appearing from the lumen of the jejunum after 30 minutes ischemia and more than 60 minutes ischemia, shows sheets of enterocytes (100x) (A), containing many M30 positive cells (arrows) in red (AEC) (100x) (B).

\section{Discussion}

This study for the first time provides insight in the sequence of events taking place during intestinal ischemia-reperfusion (IR) in man. Intestinal IR is often encountered in clinical practice, not only in patients presenting with abdominal complaints, but also during e.g. exercise, major surgery and trauma [1-4,8]. Patients undergoing pancreatico-duodenectomy enabled us to create the first human experimental model allowing detailed studies of intestinal IR induced 
cell damage, because in this procedure a variable length of healthy jejunum is removed as part of the standard operative procedure. This controlled model completely equals the interruption of blood flow to the small intestine, which often occurs during repair of a (thoraco-) abdominal aneurysm of the aorta, cardiopulmonary bypass, and intestinal transplantation.

Our study reveals that three phenomena can be distinguished following 30 minutes of human intestinal ischemia. Firstly, during the ischemic period the epithelial integrity remained seemingly intact, while subepithelial spaces appeared by detachment of the basement membrane from the enterocytes without any indication for the onset of apoptosis. The presence of I-FABP staining in the subepithelial spaces strongly indicates loss of membrane integrity of the intestinal epithelial cells at the site of formation of subepithelial spaces. These data are in line with the rapid increase of arteriovenous concentration differences of I-FABP immediately upon ischemia. Secondly, within 30 minutes reperfusion, desquamation of the intestinal epithelium at the tip of the villi was observed into the lumen, accompanied by apoptosis of mature epithelial cells. Thirdly, within 60 minutes reperfusion, a fully intact epithelial lining was found, indicating rapid restoration, whereas the apoptotic enterocytes had been shedded in the lumen. This reaction of the intestinal villi upon ischemia reperfusion, which collectively is called restitution, was also found in previous in vitro and in vivo animal studies after various insults, as recently reviewed by Blikslager et al ${ }^{19}$. However, the consequences of intestinal IR in animal studies vary largely and seem to be dependent on the animal model, the animal strain, preoperative care (e.g. starvation/fasting, premedication) and duration of ischemia or hypoperfusion ${ }^{17-19}$.

Intestinal epithelial restitution is hypothesized to be directed at repairing the epithelial defect, which originates from the detachment of damaged enterocytes. The appearance of subepithelial spaces during ischemia is the actual start of the regeneration and aimed at reducing the effective size of the injured area. The development of subepithelial spaces after splanchnic hypoperfusion in animal studies was initially interpreted as an accumulation of cytoplasmic fluid from ischemic damaged cells ${ }^{17}$. Later, evidence was provided by an in vitro model of chemically-induced injury to guinea pig ileal epithelium that the subepithelial space resulted from the retraction of the basement membrane by contraction of myofibroblasts ${ }^{29}$. This retraction leads to a smaller and denser lamina propria accompanied by some seepage of fluid from the lamina propria cells into the newly formed subepithelial space. This is in line with our observations that the subepithelial spaces developing after 30 minutes of ischemia were associated with a dense lamina propria and a liquid-containing 


\section{Chapter 5}

space between the basement membrane and enterocytes. Immediately after the start of the reperfusion, the I-FABP staining of the subepithelial space strongly indicates a loss of enterocyte membrane integrity, resulting in leakage of I-FABP into the subepithelial space. In line, we observed immediately upon ischemia strongly elevated arteriovenous concentration gradients of I-FABP, reflecting the membrane integrity loss of the epithelial cells of the villus. Therefore, we hypothesize that the enterocyte cell membrane must have lost its integrity during ischemia.

The mature enterocytes at the tip of the villus are the cells that are most susceptible to IR ${ }^{33}$. This has classically been explained by their constant state of hypoxia due to the counter current exchange mechanism of oxygen in the villus microvasculature, wherein oxygen from arterial blood entering the villus diffuses across to neighbouring venules travelling from the tip down toward the base of the villus ${ }^{33}$. As a result of this phenomenon, a steep oxygen gradient is present in the intestinal villus with substantially lower oxygen tensions in the villus tip than at the crypt ${ }^{33}$. In addition, desquamation occurs of the damaged mature enterocytes from the tip of the villi, resulting in shedding of sheets of epithelial cells into the lumen. Apoptosis is the major form of cell death observed after the onset of reperfusion and at the villus tip. Apoptosis of detaching enterocytes after intestinal IR is potentially caused by loss of contact between the epithelial cells and extracellular matrix, and between neighbouring epithelial cells ('anoikis') ${ }^{20,31,34}$. Extracellular matrix molecules provide a constant survival signal to epithelial cells via, 1 -integrins and cadherins as well as their adhesion-mediated signalling pathways including focal adhesion kinase (p125fak), phosphatidylinositol 3'-kinase (PI3-K)/Akt and mitogen-activated protein kinase (MAPK; p38) ${ }^{34-37}$. However, senescent enterocytes gradually loosen cell anchorage by changes of integrin expression, cadherin binding and its signalling pathways, making them prone to be shedded from the villus tip at the end of their lifespan, a mechanism crucial for maintenance of homeostasis in this epithelium ${ }^{35-37}$. Moreover, acyl-coenzyme A synthetase 5, which is solely expressed at the villus tip, is able to sensitize epithelial cells to apoptosis specifically triggered by the death ligand TRAIL ${ }^{38}$. Therefore, mature enterocytes are in an anti-adhesive and pro-apoptotic state when they reach the villus tip, culminating in the physiologic shedding ${ }^{39}$. This may contribute to the rapid onset of apoptosis that we observed in the mature and damaged enterocytes after the ischemic period, upon reperfusion.

The ultimate reparative response to the lost epithelial continuity was observed within one hour of reperfusion, consisting of a complete resealing of the epithelial defect overlying the basement membrane by viable, ZO-1 and FABP 
containing, epithelial cells. The presence of tight junctions and endogenous cytosolic proteins in the repaired epithelial cells of the villus tip indicates that the epithelial lining is rapidly restored, which is of importance to reduce the opportunity for luminal microbiota and their products to translocate. The question remains whether this also leads to a reestablishment of the full barrier function, which will be assessed in future using physiological measurements by tracer or electrophysiological studies. Previous studies reported that the migration of epithelial cells involves alteration of enterocyte shape and phenotype, including extension of the plasma membrane in the direction of cell migration and assembly of new focal contacts at the leading edge ${ }^{19,40,41}$. Epithelial migration during restitution is enhanced by luminal contents and a variety of systemic and local factors, including trefoil peptides, polyamines and transforming growth factor, 19,42 .

The clearing mechanism of IR-induced injured cells is unique to the intestine. In other organs or tissues, such as heart, brain, kidney, liver and skeletal muscle, IR-induced damaged and death cells remain in situ, since they cannot be removed from the organs or tissues and cleared from the body. In such organs, an inflammatory response is elicited amongst others to clear away the injured and death cells after IR 10,13-15,43. Subject of further studies is the investigation of the inflammatory response in our controlled, experimental model of human IR, which enabled us to clarify a gut-specific protective mechanism for IR-induced damaged cells. Our results might explain why the gut can tolerate 30 minutes of ischemia in events as exercise, trauma, repair of a (thoraco-) abdominal aneurysm of the aorta, and cardiopulmonary bypass.

\section{References}

1. (2000) American Gastroenterological Association Medical Position Statement: guidelines on intestinal ischemia. Gastroenterology 118: 951-953.

2. Oldenburg WA, Lau LL, Rodenberg TJ, Edmonds HJ, Burger CD (2004) Acute mesenteric ischemia: a clinical review. Arch Intern Med 164: 1054-1062.

3. Jeukendrup AE, Vet-Joop K, Sturk A, Stegen JHJC, Senden J, et al. (2000) Relationship between gastro-intestinal complaints and endotoxaemia, cytokine release and the acute-phase reaction during and after a long-distance triathlon in highly trained men. Clin Science 98: 47-55.

4. Perko MJ, Nielsen HB, Skak C, Clemmesen JO, Schroeder TV, et al. (1998) Mesenteric, coeliac and splanchnic blood flow in humans during exercise. J Physiol 513: 907-913. 


\section{Chapter 5}

5. Rokyta R, Matejovic M, Novak I, Zeman V, Krouzecky A, et al. (2002) Submaximal exercise in healthy volunteers: the relationship between gastric mucosal and systemic energy status. Pflügers Arch - Eur J Physiol 443: 852-857.

6. Veenstra R, Geelkerken R, Verhorst P, Huisman A, Kolkman J (2007) Acute stress-related gastrointestinal ischemia. Digestion 75: 205-207.

7. Fukatsu K, Sakamoto S, Hara E, Ueno C, Maeshima Y, et al. (2006) Gut ischemiareperfusion affects gut mucosal immunity: a possible mechanism for infectious complications after severe surgical insults. Crit Care Med 34: 182-187.

8. Mallick IH, Yang W, Winslet MC, Seifalian AM (2004) Ischemia-reperfusion injury of the intestine and protective strategies against injury. Dig Dis Sci 49: 1359-1377.

9. Semenza GL (2000) Series introduction: tissue ischemia: pathophysiology and therapeutics. J Clin Invest 106: 613-614.

10. Lee JM, Grabb MC, Zipfel GJ, Choi DW (2000) Brain tissue responses to ischemia. J Clin Invest 106: 723-731.

11. Carden DL, Granger DN (2000) Pathophysiology of ischaemia-reperfusion injury. J Pathol 190: 255-266.

12. Matzinger P (2007) Friendly and dangerous signals: is the tissue in control? Nat Immunol 8: 11-13.

13. Jaeschke H (2003) Molecular mechanisms of hepatic ischemia-reperfusion injury and preconditioning. Am J Physiol Gastrointest Liver Physiol 284: G15-26.

14. Blaisdell FW (2002) The pathophysiology of skeletal muscle ischemia and the reperfusion syndrome: a review. Cardiovasc Surg 10: 620-630.

15. Daemen MA, de Vries B, Buurman WA (2002) Apoptosis and inflammation in renal reperfusion injury. Transplantation 73: 1693-1700.

16. Arumugam TV, Shiels IA, Woodruff TM, Granger DN, Taylor SM (2004) The role of the complement system in ischemia-reperfusion injury. Shock 21: 401-409.

17. Chiu CJ, McArdle AH, Brown R, Scott HJ, Gurd FN (1970) Intestinal mucosal lesion in low-flow states. I. A morphological, hemodynamic, and metabolic reappraisal. Arch Surg 101: 478-483.

18. Park PO, Haglund U, Bulkley GB, Falt K (1990) The sequence of development of intestinal tissue injury after strangulation ischemia and reperfusion. Surgery 107: 574-580.

19. Blikslager AT, Moeser AJ, Gookin JL, Jones SL, Odle J (2007) Restoration of barrier function in injured intestinal mucosa. Physiol Rev 87: 545-564.

20. Ikeda H, Suzuki Y, Suzuki M, Koike M, Tamura J, et al. (1998) Apoptosis is a major mode of cell death caused by ischaemia and ischaemia/reperfusion injury to the rat intestinal epithelium. Gut 42: 530-537.

21. Park PO, Haglund U (1992) Regeneration of small bowel mucosa after intestinal ischemia. Crit Care Med 20: 135-139. 
22. Hart ML, Ceonzo KA, Shaffer LA, Takahashi K, Rother RP, et al. (2005) Gastrointestinal ischemia-reperfusion injury is lectin complement pathway dependent without involving C1q. J Immunol 174: 6373-6380.

23. Chen LW, Egan L, Li ZW, Greten FR, Kagnoff MF, et al. (2003) The two faces of IKK and NF-kappaB inhibition: prevention of systemic inflammation but increased local injury following intestinal ischemia-reperfusion. Nat Med 9: 575-581.

24. Panes J, Granger DN (1998) Leukocyte-endothelial cell interactions: molecular mechanisms and implications in gastrointestinal disease. Gastroenterology 114: 10661090.

25. Haglund U, Hulten L, Ahren C, Lundgren O (1975) Mucosal lesions in the human small intestine in shock. Gut 16: 979-984.

26. Kerstens HM, Robben JC, Poddighe PJ, Melchers WJ, Boonstra H, et al. (2000) AgarCyto: a novel cell-processing method for multiple molecular diagnostic analyses of the uterine cervix. J Histochem Cytochem 48: 709-718.

27. Pelsers MM, Hermens WT, Glatz JF (2005) Fatty acid-binding proteins as plasma markers of tissue injury. Clin Chim Acta 352: 15-35.

28. Fanning AS, Jameson BJ, Jesaitis LA, Anderson JM (1998) The tight junction protein ZO1 establishes a link between the transmembrane protein occludin and the actin cytoskeleton. J Biol Chem 273: 29745-29753.

29. Powell DW, Mifflin RC, Valentich JD, Crowe SE, Saada JI, et al. (1999) Myofibroblasts. II. Intestinal subepithelial myofibroblasts. Am J Physiol 277: C183-201.

30. Kuhn K (1995) Basement membrane (type IV) collagen. Matrix Biol 14: 439-445.

31. Frisch SM, Francis H (1994) Disruption of epithelial cell-matrix interactions induces apoptosis. J Cell Biol 124: 619-626.

32. Leers MP, Kolgen W, Bjorklund V, Bergman T, Tribbick G, et al. (1999) Immunocytochemical detection and mapping of a cytokeratin 18 neo-epitope exposed during early apoptosis. J Pathol 187: 567-572.

33. Blikslager AT (2008) Life in the gut without oxygen: adaptive mechanisms and inflammatory bowel disease. Gastroenterology 134: 346-348.

34. Hofmann C, Obermeier F, Artinger M, Hausmann M, Falk W, et al.(2007) Cell-cell contacts prevent anoikis in primary human colonic epithelial cells. Gastroenterology 132: $587-600$.

35. Fouquet S, Lugo-Martinez VH, Faussat AM, Renaud F, Cardot P, et al. (2004) Early loss of E-cadherin from cell-cell contacts is involved in the onset of Anoikis in enterocytes. J Biol Chem 279: 43061-43069.

36. Vachon PH, Harnois C, Grenier A, Dufour G, Bouchard V, et al. (2002) Differentiation state-selective roles of p38 isoforms in human intestinal epithelial cell anoikis. Gastroenterology 123: 1980-1991. 


\section{Chapter 5}

37. Dufour G, Demers MJ, Gagne D, Dydensborg AB, Teller IC, et al. (2004) Human intestinal epithelial cell survival and anoikis. Differentiation state-distinct regulation and roles of protein kinase B/Akt isoforms. J Biol Chem 279: 44113-44122.

38. Gassler N, Roth W, Funke B, Schneider A, Herzog F, et al. (2007) Regulation of enterocyte apoptosis by acyl-CoA synthetase 5 splicing. Gastroenterology 133: 587598.

39. Bullen TF, Forrest S, Campbell F, Dodson AR, Hershman MJ, et al. (2006) Characterization of epithelial cell shedding from human small intestine. Lab Invest 86: 1052-1063.

40. Moore R, Carlson S, Madara JL (1989) Rapid barrier restitution in an in vitro model of intestinal epithelial injury. Lab Invest 60: 237-244.

41. Moore R, Madri J, Carlson S, Madara JL (1992) Collagens facilitate epithelial migration in restitution of native guinea pig intestinal epithelium. Gastroenterology 102: 119-130.

42. Podolsky DK (1997) Healing the epithelium: solving the problem from two sides. J Gastroenterol 32: 122-126.

43. Yellon DM, Hausenloy DJ (2007) Myocardial reperfusion injury. N Engl J Med 357: 1121-1135. 


\subsection{Mannose-binding lectin null alleles are associated with preserved epithelial cell integrity following intestinal ischemia reperfusion in man}

Robert A. Matthijsen, Joep P. M. Derikx, Rudi Steffensen, Ronald M. van Dam, Cornelis H. C. Dejong, Wim A. Buurman Molecular Immunology, in press. 


\section{Chapter 5}

\section{INTRODUCTION}

Intestinal ischemia reperfusion (I-IR) injury has a multifactorial etiology, involving activation of complement. Mannose-binding lectin (MBL) is considered to be an important initiating complement component with considerable variation in activating properties and plasma levels between individuals. These variations are caused by three first exon mutations (at codons 52, 54 and 57) and polymorphisms in the promoter region (at position -550 $(\mathrm{H} / \mathrm{L})$ and $-221(\mathrm{X} / \mathrm{Y}))$ of the structural gene $(\mathrm{mb} / 2) .{ }^{1}$ Patients were classified according to their first exon structural genotype; $\mathrm{A} / \mathrm{A}, \mathrm{A} / \mathrm{O}$ and $\mathrm{O} / \mathrm{O}$, where any of the three first exon variations is coded as O. Homozygous or heterozygous carriers of variant mbl2 alleles express a disrupted MBL protein without apparent complement activating properties that confers an increased susceptibility to infectious diseases. ${ }^{2}$ Theoretically, this increased susceptibility to infection would be anticipated to be eliminated as a consequence of evolutionary pressure. Considering the high prevalence of nearly $40 \%$ of $\mathrm{MBL}$ null variants in the Caucasian population, a broader role of $M B L$ with a significant biological advantage for such variant alleles should be considered.

\section{Patients and methods}

During a pylorus preserving pancreatico-duodenectomie (PPPD) part of the small bowel is resected, providing the opportunity to study ischemia reperfusion induced cell damage in a harmless human IR model of the jejunum. A six centimeter long segment of the jejunum was subjected to 30 minutes of ischemia and reperfusion (mean 128 minutes (30-240)). Ischemia was induced using two non-traumatic vascular clamps, bilaterally clamping both afferent and efferent branches of the vasculature. Ischemia was objectivated macroscopically by discoloration of the jejunum outer wall and termination of the peristalsis of the jejunum. Following thirty minutes of ischemia, reperfusion was restored by removing the vascular clamps, immediately checking reperfusion signs (regaining of color, restoration of gut motility and sampling of the venous outflow). For the remainder of the procedure the IR specimen was kept in the abdominal cavity. Local ethical approval and informed consent were obtained. 29 Individuals were consecutively included. Due to incomplete reperfusion 2 individuals were excluded before analysis. Data from 27 remaining individuals were analyzed by one-way analysis of variance (ANOVA) and unpaired two-tailed Students T-test.

As a measure for intestinal epithelial cell damage arteriovenous plasma differences in intestinal-fatty acid binding protein (I-FABP) levels were assessed 
before ischemia, directly upon and every thirty minutes during reperfusion in afferent $(A)$ and efferent $(V)$ mesenteric blood samples drawn from the isolated bowel segment $(\Delta(\mathrm{V}-\mathrm{A})){ }^{3}$ By subtracting arterial I-FABP concentration from detected venous levels, either release or consumption can be easily assessed. In addition, arteriovenous plasma differences in MBL levels were established and the MBL genotype was assessed retrospectively by means of quantitative-PCR analysis, using sequence-specific primers. ${ }^{4}$

\section{ReSULTS}

The amount of epithelial cell damage varied significantly between the carriers of different mbl2 genotypes (ANOVA, $p=0.02$ ), with peak levels in I-FABP release detected immediately upon reperfusion in every included individual. Homozygous wildtype individuals ( $\mathrm{A} / \mathrm{A}, \mathrm{N}=14 ; 64 \%$ men) showed a maximal mean $( \pm$ SEM) I-FABP release of $4,960 \mathrm{pg} / \mathrm{ml}( \pm 780 \mathrm{pg} / \mathrm{ml})$ immediately after reperfusion. Interestingly, heterozygous ( $\mathrm{A} / \mathrm{O}, \mathrm{N}=11 ; 55 \%$ men) and homozygous $(\mathrm{O} / \mathrm{O}, \mathrm{N}=2$; two women) carriers of variant $\mathrm{MBL}$ alleles showed maximal mean I-FABP release of $2,560 \mathrm{pg} / \mathrm{ml}( \pm 690 \mathrm{pg} / \mathrm{ml})(\mathrm{p}=0.03$ compared to $\mathrm{A} / \mathrm{A})$ and $240 \mathrm{pg} / \mathrm{ml}( \pm 60 \mathrm{pg} / \mathrm{ml})(\mathrm{p}=0.04$ compared to $\mathrm{A} / \mathrm{A})$, respectively (Fig. 1). I-FABP levels between $\mathrm{A} / \mathrm{O}$ and $\mathrm{O} / \mathrm{O}$ individuals were statistically similar $(p=0.19)$. The data were corroborated by histological analysis of tissue samples collected at the end of reperfusion. Large amounts of epithelial cellular debris in $\mathrm{A} / \mathrm{A}$ individuals, whereas lesser debris was observed in $\mathrm{A} / \mathrm{O}$ and $\mathrm{O} / \mathrm{O}$ genotype carriers (Fig. 1).

The data indicate that MBL influences intestinal epithelial cell integrity in an immediate and non-complement dependent manner during ischemia, considering the different peak I-FABP levels immediately upon reperfusion. Analysis of the MBL levels in afferent and efferent blood samples from the reperfused ischemic jejunum during both early and prolonged reperfusion, revealed no MBL consumption (Fig. 2. $p=0.19$ ). This suggests that activation and binding of plasma $\mathrm{MBL}$ during reperfusion does not occur. The data indicate a protective role for the $\mathrm{MBL}$ variant gene products, possibly on the intracellular level. 


\section{Chapter 5}

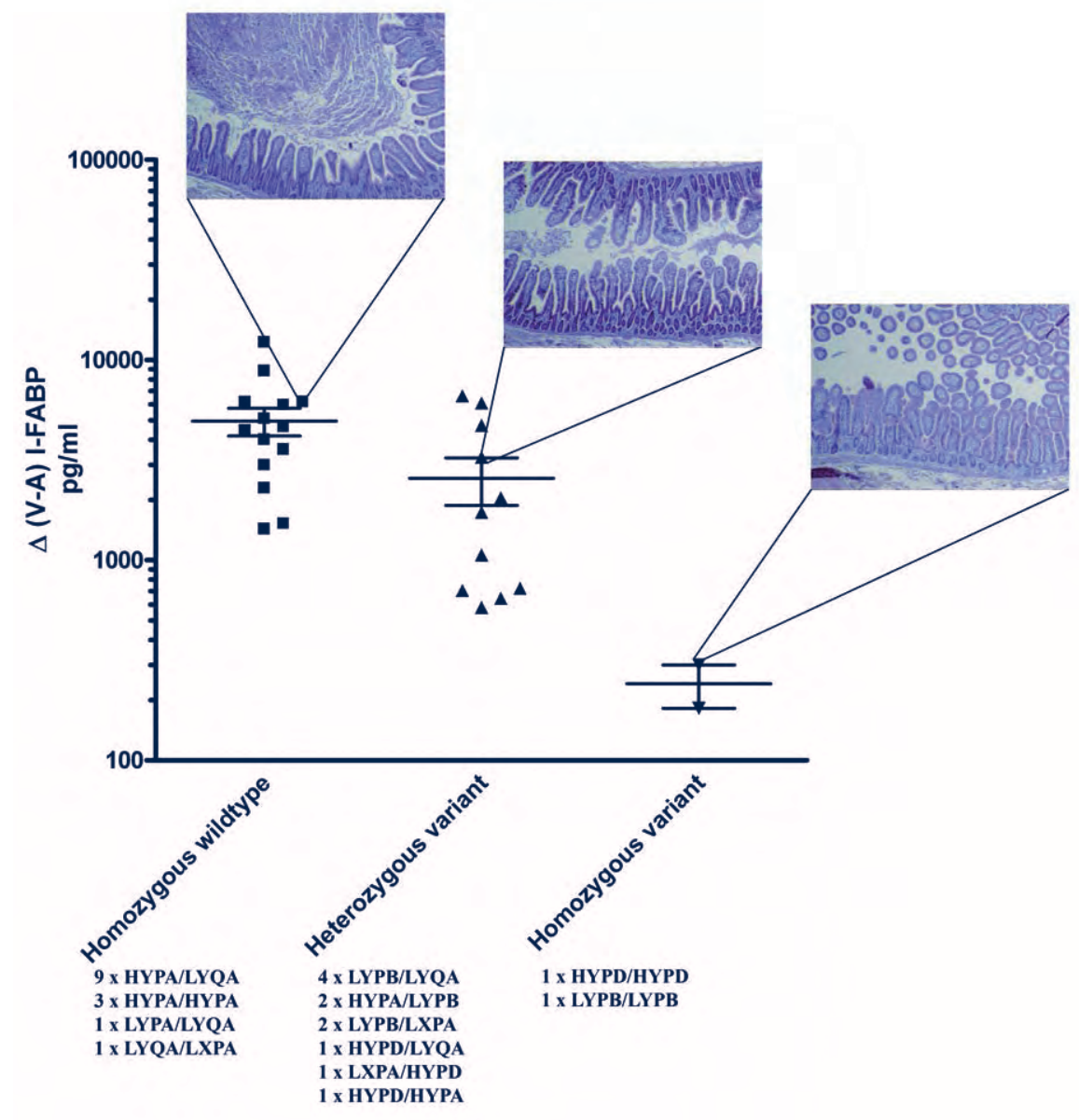

Figure 1: Intestinal epithelial damage.

Maximal epithelial damage, represented by peak I-FABP levels observed at the very beginning of reperfusion, was calculated from the arteriovenous difference in I-FABP levels across an isolated bowel segment before and after ischemia $(\Delta(\mathrm{V}-\mathrm{A}))$. Epithelial damage was virtually absent in homozygous ( $p=0.03$ ) and reduced in heterozygous $(p=0.04$ ) carriers of variant mbl2 alleles when compared to their homozygous wildtype counterparts. The difference between heterozygous variant and homozygous variant individuals was statistically similar $(p=0.19)$. Integral I-FABP release correlated with individual peak I-FABP levels in response to IR. Genotypes have been listed for all carriers. Microphotographs demonstrate cellular debris observed after IR in the intestinal lumen. Original magnification 100x. 


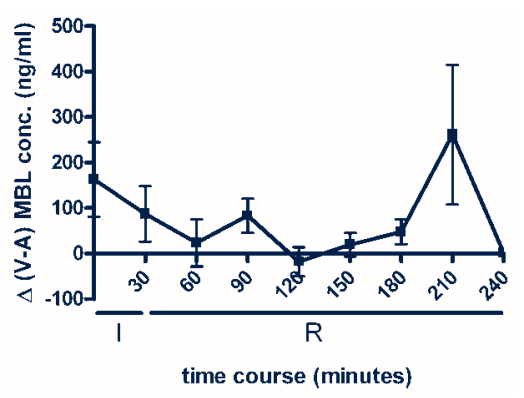

Figure 2: Plasma MBL

No consumption of plasma MBL was detected when analyzing arteriovenous concentration differences $(\Delta(\mathrm{V}-\mathrm{A}))$ over the isolated jejunum segment during IR $(\mathrm{p}=0.19)$.

\section{Discussion}

Over time, $\mathrm{mb} / 2$ variant genotypes have been considered both advantageous as well as disadvantageous to human evolution. On the one hand, variant mbl2 alleles are suggested to be protective against the development of leprosy. ${ }^{5} \mathrm{On}$ the other hand, variant mbl2 alleles have been linked to an increased risk of developing, atherosclerosis, ${ }^{6}$ thrombosis ${ }^{7}$ and susceptibility to infectious diseases. ${ }^{2}$ Synthesis of MBL is mainly attributed to the liver as well as the small intestine and results in two MBL forms, namely plasma and intracellular MBL. ${ }^{8,9}$ Hitherto, no in vivo function of intracellular MBL has been determined. However, convincing in vitro data show the ability of MBL to inhibit meprins, zinc metalloproteinases associated with IR induced cell damage and abundantly present in intestinal epithelial cells. ${ }^{10,11}$ Taken together the data might point towards ischemia protective properties of variant and intracellular $M B L$, associated with far-reaching beneficial qualities without an apparent immunological function.

If confirmed in a larger patient cohort, our data may explain why mutations in the $\mathrm{MBL}$ coding sequence, resulting in a defective immune regulatory function as well as reduced levels of one of our prominent innate regulators of immunity, have survived selection pressure during human evolution.

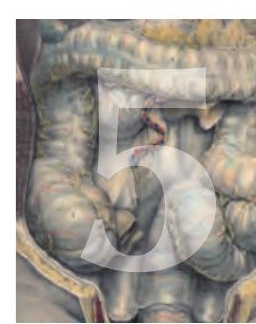




\section{Chapter 5}

\section{RefERENCES}

1. Turner MW, Hamvas RM. Mannose-binding lectin: structure, function, genetics and disease associations. Rev Immunogenet 2000;2(3):305-22.

2. Summerfield JA, Ryder S, Sumiya M, Thursz M, Gorchein A, Monteil MA, et al. Mannose binding protein gene mutations associated with unusual and severe infections in adults. Lancet 1995;345(8954):886-9.

3. Kanda T, Fujii H, Tani T, Murakami H, Suda T, Sakai Y, et al. Intestinal fatty acid-binding protein is a useful diagnostic marker for mesenteric infarction in humans. Gastroenterology 1996;110(2):339-43.

4. Henckaerts L NK, Steffensen R, Wouters J, Milants I, Vanhorebeek I, Langouche L, Vermeire S, Rutgeerts P, Thiel S, Wilmer A, Hansen TK, Berghe GV. Polymorphisms In Innate Immunity Genes Predispose To Bacteremia And Death In The Medical ICU. JCI (in press).

5. Dornelles LN, Pereira-Ferrari L, Messias-Reason I. Mannan-binding lectin plasma levels in leprosy: deficiency confers protection against the lepromatous but not the tuberculoid forms. Clin Exp Immunol 2006;145(3):463-8.

6. Madsen HO, Videm V, Svejgaard A, Svennevig JL, Garred P. Association of mannosebinding-lectin deficiency with severe atherosclerosis. Lancet 1998;352(9132):959-60.

7. Ohlenschlaeger T, Garred P, Madsen HO, Jacobsen S. Mannose-binding lectin variant alleles and the risk of arterial thrombosis in systemic lupus erythematosus. N Eng/ J Med 2004;351(3):260-7.

8. Seyfarth J, Garred P, Madsen HO. Extra-hepatic transcription of the human mannosebinding lectin gene $(\mathrm{mbl} 2)$ and the MBL-associated serine protease 1-3 genes. Mol Immunol 2006;43(7):962-71.

9. Nonaka M, Ma BY, Ohtani M, Yamamoto A, Murata M, Totani K, et al. Subcellular localization and physiological significance of intracellular mannan-binding protein. J Biol Chem 2007;282(24):17908-20.

10. Hirano M, Ma BY, Kawasaki N, Okimura K, Baba M, Nakagawa T, et al. Mannanbinding protein blocks the activation of metalloproteases meprin alpha and beta. J Immunol 2005;175(5):3177-85.

11. Carmago S, Shah SV, Walker PD. Meprin, a brush-border enzyme, plays an important role in hypoxic/ischemic acute renal tubular injury in rats. Kidney Int 2002;61(3):95966. 


\subsection{Enterocyte shedding and epithelial lining repair following ischemia of the human small intestine attenuates inflammation}

Robert A. Matthijsen, Joep P. M. Derikx, Dian Kuipers, Ronald M. van Dam, Cornelis H. C. Dejong, Wim A. Buurman Submitted. 


\section{Chapter 5}

\section{Abstract}

\section{Background and aims}

Recently, we observed that in the human small intestine ischemia is rapidly followed by sloughing of damaged epithelial cells and repair of the epithelial lining. This phenomenon ensures a rapid loss of apoptotic and necrotic cells. Classically, following ischemia and reperfusion (IR), damaged cells trigger an inflammatory response resulting in enhanced tissue damage. This study investigates whether the observed shedding of ischemically damaged epithelial cell lining cells affects IR induced inflammation in the human small gut.

\section{Methods}

Using a newly developed IR model of the human small intestine, the inflammatory response is studied on cellular, protein and mRNA level. 30 Patients were consecutively included in our tertiary care hospital. Part of the jejunum was subjected to 30 minutes of ischemia and reperfusion. Mean reperfusion time was 123 (30-240) minutes. Ethical approval and informed consent were obtained.

\section{Results}

Gut barrier failure, indicated by increased LPS concentration in venous effluent blood, was not observed during IR $(p=0.18)$. Release of TNF $\alpha$ from the reperfused tissue could not be detected. Additionally, gene expression of $\mathrm{HO}$ 1, IL-6, IL-8 did not alter. No endothelial activation markers or increased numbers of inflammatory cells $(p=0.71)$ were detected in the reperfused tissue. Complement was not activated, indicated by C3 deposition ( $\mathrm{p}=0.14)$. However, in response to anoxia, HIF- $1 \alpha$ gene expression doubled $(p=0.02)$ whereas C3 gene expression increased 4 -fold ( $p=0.01$ ) over the course of IR.

\section{Conclusions}

The human small intestine is classically considered vulnerable to IR. However, these are first human in vivo data that illustrate the jejunum's unique ability to prevent IR induced inflammation by shedding of damaged enterocytes. This understanding will aid current and future developments and diagnostics within the field of gastroenterology. 


\section{INTRODUCTION}

Recently, we demonstrated the ability of the human small intestine to rapidly restore its epithelial architecture after massive epithelial sloughing and gut barrier damage in response to a thirty minutes ischemic episode and reperfusion. ${ }^{1}$ Interestingly, analysis of the reperfused intestinal segment demonstrated that the epithelial brush border and epithelial tight junctions were restored within the first hour of reperfusion.

The presence of apoptosis or cellular damage in reperfused ischemic organs has cardinal implications in the pathogenesis of ischemia reperfusion. ${ }^{2},{ }^{3}$ Massive apoptosis and subsequent necrosis are considered to be functionally involved in the induction of inflammation after organ ischemia, since therapeutic strategies aimed at preventing IR induced apoptosis can ameliorate IR induced inflammation. ${ }^{4-7}$ Additionally, numerous experimental studies have shown that the inflammatory response following IR mediates the development of additional reperfusion injury, greatly compromising organ function. Different strategies aimed at preventing IR induced inflammation can effectively reduce ultimate organ damage. ${ }^{8}$ The inflammatory response induced by IR is essentially characterized by different contributors. Early after cellular injury various cytokines, such as tumor necrosis factor- $\alpha$, interleukin-1, $-6,-8$ or -10 and others, are expressed. ${ }^{9,} 10$ Together with the rapid expression of adhesion molecules by activated endothelial cells, the activation, adhesion and sequestration of polymorphonuclear neutrophils (PMN) into the affected tissue is induced. ${ }^{11}$ By locally releasing myeloperoxidase (MPO), reactive oxygen species and many more of their pro-inflammatory constituents, infiltrating PMN contribute substantially to IR induced inflammation. ${ }^{12,13}$ Additionally the complement system is activated, thereby triggering to formation of reactive complement split products known as anaphylatoxins, which induce additional PMN chemotaxis and cause organ damage. ${ }^{14,15}$ Ultimately, complement activation will lead to cell damage by membrane attack complex (MAC) formation. ${ }^{15,16}$ Normally, under healthy conditions, these innate immune constituents protect us from harm by orchestrating a well mounted attack on invading microorganisms, but when faced with extensive I/R injury, sufficient means of control seem absent. In this respect, interventions aimed at preventing complement activation, ${ }^{17}$ MAC formation, ${ }^{18}$ expression of adhesion molecules ${ }^{19}$ or PMN sequestration ${ }^{20,21}$ all prevent additional reperfusion injury.

Taken together, the presence of apoptotic and necrotic cells following ischemia reperfusion of the small intestine is of critical importance in the development of an inflammatory reaction that contributes to tissue injury. From 


\section{Chapter 5}

this we hypothesized that the remarkable ability of the human small intestine to effectively shed apoptotic cells, losing in this way an important endogenous inflammatory trigger, serves as a rescue mechanism, unique to the intestine, that potentially attenuates the development of an intense IR induced inflammatory reaction. Therefore, we studied time related characteristics of IR induced inflammation of the human small intestine in a newly developed human ischemia reperfusion model of the jejunum. The results indicate that reperfused ischemic jejunum effectively discards apoptotic and damaged epithelium, restores the epithelial gut barrier and thereby prevents the development of a local inflammatory response.

\section{MATERIALS AND METHODS}

\section{Patients and ethics}

The study was approved by the local ethical committee. Written consent of all patients was obtained before inclusion. We consecutively included 30 patients over 18 years of age. The included patients were scheduled to undergo pancreatic surgery; twenty eight underwent a pylorus preserving pancreaticoduodenectomy (Whipple's procedure) for benign or malignant pancreas disease and two a Frey procedure for chronic pancreatitis. The small intestine samples from every included patient were macroscopically assessed for evident signs of pathology during operation and before IR procedure. Due to an incomplete reperfusion 2 patients were excluded before further analysis.

\section{Study model}

Patients were anesthetized according to institutional routines using isoflurane and propofol. Operation was initiated by a subcostal bilateral incision. OmniTracts were used to improve exposure. Per operatively echographic confirmation of diagnosis and disease progression was obtained. Six centimeters of the small intestine (jejunum) resection specimen, just proximal to the ligament of Treitz at the duodenal-jenunal transition, were mobilized and transsected using proximal and distal universal straight endo-GIA (GIA 6038S, Covidien, MA). Both afferent and efferent mesenteric vascular structures were previously dissected from their surrounding tissues and remain intact. To prevent bowel rotation, compromising segmental blood flow, the isolated segment was fixed to both the proximal and distal small bowel continuum. Ischemia was induced using two non-traumatic vascular clamps, bilaterally clamping both afferent and efferent branches of the vasculature. Ischemia was objectivated macroscopically by discoloration of the jejunum outer wall and termination of the peristalsis of the studied jejunum. 
Following thirty minutes of ischemia, reperfusion was restored by removing the vascular clamps, immediately checking reperfusion signs (regaining of color, restoration of gut motility and sampling of the venous outflow). For the remainder of the procedure the IR specimen was kept in the abdominal cavity. Serial blood samples were drawn before ischemia, on reperfusion and every thirty minutes during reperfusion from the afferent vein draining the isolated and ischemically damaged jejunum. Timed to the venous blood samples, blood from the arteria radialis was collected to represent arterial intestinal inflow. The total reperfusion time was determined by the duration of the surgical procedure, with a maximum of 240 minutes. Reperfused ischemic intestine samples were obtained from every patient $(n=28)$. Healthy jejunum samples, serving as internal controls, were obtained from 12 patients.

\section{Immunohistochemistry}

Paraffin-embedded sections from healthy and IR damaged jejunum were prepared. Endothelial adhesion molecules E-selectin (CD-62-E, monoclonal antibody ENA1, kindley provided by Hycult Biotechnology (Hbt, Uden, the Netherlands)), intercellular adhesion molecule-1 (ICAM-1, monoclonal antibody HM1, Hbt) and mucosal vascular addressin cell adhesion molecule 1 (MADCAM-1, monoclonal antibody 314G8, Hbt), neutrophils by myeloperoxidase staining (polyclonal antibody, A0398, DakoCytomation, Glostrup, Denmark) or human neutrophil defensin 1-3 (monoclonal antibody, D21, Hbt) and complement component C3c (A0062, DakoCytomation) were immunostained on paraffin embedded sections. The sections were deparaffinized, rehydrated and blocked for 1 hour at room temperature using 5\% BSA in phosphate buffered saline (PBS) or tris buffered saline (TBS) for HM1 and MPO. Properly diluted primary antibodies were incubated in PBS or TBS supplemented with $0.1 \%$ BSA for 1 hour at room temperature. After washing, specific antibody binding was detected using specific peroxidase conjugated secondary antibodies (Jackson Immunoresearch, West Grove, PA) or a biotinilated swine anti rabbit secondary antibody (E0432, DakoCytomation) for detecting MPO specific binding. Next, signal enhancement was achieved by peroxidase conjugated avidin biotin complexes. Staining was visualized by 3-amino-9-ethylcarbazole (AEC) followed by a hematoxylin (Sigma, St. Louis, MO) nuclear counterstaining.

\section{ELISA}

Arteriovenous concentration differences in plasma I-FABP levels were determined by Enzyme-Linked ImmunoSorbent Assay (ELISA) before and after 


\section{Chapter 5}

ischemia to measure intestinal mucosal cell damage. ${ }^{22}$ The I-FABP ELISA (HK406, Hbt) was performed in accordance to the manufacturer's guidelines. IFABP release was determined for every included patient.

Myeloperoxidase (MPO) was measured in tissue samples that were homogenyzed according to the previously described western blot protocol. MPO was measured using a commercially available solid-phase ELISA kit based on the sandwich priciple (Hbt) in aliquots containing equal amounts $(10 \mu \mathrm{g})$ of total protein. The assay was performed in accordance to the manufacturer's guidelines.

Arteriovenous concentration differences in plasma tumor necrosis factor- $\alpha$ (TNF $\alpha$ ) levels were determined by ELISA before and after ischemia as well as during reperfusion. The TNF $\alpha$ ELISA was preformed as described before. ${ }^{23}$

Arteriovenous concentration differences in plasma lipopolysacharide (LPS) levels were determined using a Limulus Amebocyte Lysate (LAL) assay before and after ischemia as well as during reperfusion. The LAL assay (HIT 302, Hbt) was performed in accordance to the manufacturer's guidelines. Plasma LPS was determined in samples collected at pre-fixed time points.

\section{Western Blot}

Western blot analyses for human C3 deposition on reperfused ischemic tissue samples and healthy controls was performed as described before, with minor modifications. ${ }^{4}$ Jejunal samples were homogenized in lysis buffer $(200 \mathrm{mM}$ $\mathrm{NaCl}, 10 \mathrm{mM}$ Tris base, 5mM EDTA, 10\% Glycerin, $1 \mathrm{mM}$ PMSF, $0.1 \mathrm{U} / \mathrm{ml}$ Aprotinin and $1 \mu \mathrm{g} / \mathrm{ml}$ Leupeptin). Tissue homogenates were centrifuged. The protein concentration of the different lysates was determined using Bradford analyses. Aliquots containing equal amounts $(10 \mu \mathrm{g})$ of total protein were heated and reduced, transferred to a 8\% SDS-polyacrylamide gel and blotted on an Immobilon-P polyvenylidine fluoride (PVDF) membrane (Millipore, Bedford, MA). Blocking was performed in phosphate buffered saline (PBS) containing 5\% bovine serum albumin and 0.1\% Tween-20 (Sigma). C3 was detected in PBS $0.1 \%$ BSA $0.1 \%$ Tween-20 after overnight incubation of the properly diluted rabbit anti human C3C (A0062, DakoCytomation, Glostrup, Denmark). Binding of the primary antibody was detected using a biotinilated secondary antibody to rabbit IgG (E0353, Dako) and streptavidine conjugated peroxidase. Positive bands were visualized using chemiluminescence (Supersignal (pico:femto 9:1), Pierce, Rockford, IL).

\section{Real-Time Quantitatve - PCR analysis}

Total RNA was extracted from healthy or IR damaged gut tissue samples using a SV Total RNA Isolation System according to manufacturer's guidelines 
(Promega, Madison, WI). Total cDNA was synthesized with oligo (dT) primers and Molony murine leukemia virus reverse transcriptase. Specific primers (Sigma) for amplification of hemoxygenase-1, hypoxia inducible factor $1-\alpha$, inteleukin- 6 and -8, TNF $\alpha$ and complement component C3 specific cDNA were designed using the Primer Express software package (Applied Biosystems, Foster City, CA) and tested for amplification of contaminating genomic DNA. To minimize the risk of genomic amplification the primers were positioned on different exons. Primer concentrations were optimalized and dilution curves were made from human liver cDNA standard pool to ensure an exponential Taqman amplification for each primer set. After normalization to endogenous reference genes RPLP0 and cyclophyline-A the level of expression of different proteins in healthy or IR damaged samples was determined by the comparative $\Delta \Delta \mathrm{C}_{\mathrm{T}}$ method of relative quantification. Primer specifications are listed in Table 2.

Table 1: Sequence of primers for Q-PCR analysis

\begin{tabular}{lll}
\hline Proteins & Primers & \\
\hline & Forward & Reversed \\
\hline HIF-1 $\alpha$ & 3-TGAACATAAAGTCTGCAACATGGA-5 & 5-TGAGGTTGGTTACTGTTGGTATCATATA-3 \\
H0-1 & 3-CTTCTTCACCTTCCCCAACA-5 & 5-GCTCTGGTCCTTGGTGTCAT-3 \\
TNF $\alpha$ & 3-TCAATCGGCCCGACTATCTC-5 & 5-CAGGGCAATGATCCCAAAGT-3 \\
IL-6 & 3-TCCAGGAGCCCAGCTATGAA-5 & 5-GAGCAGCCCCAGGGAGAA-3 \\
IL-8 & 3-CTGGGCGTGGCTCTCTTG-5 & 5-TTAGCACTCCTTGGCAAACTC-3 \\
C3 & 3-CCCTCATCATCTACCTGGACAAG-5 & 5-GCTGGATAAGCTCTACATTAAAGTATTGG-3 \\
\hline RPLP-0 & 3-GCAATGTTGCCAGTGTCTG-5 & 5-GCCTTGACCTTTTCAGCAA-3 \\
Cycophilin A & 3-CTCGAATAAGTTTGACTTGTGTTT-5 & 5-CTAGGCATGGGAGGGAACA-3 \\
& &
\end{tabular}

\section{Statistical analysis}

Data are expressed as means \pm SEM and were analyzed by either paired or unpaired two-tailed Student's t-test depending on sample collection or by repeated measures ANOVA (LPS release). Data were analyzed using Graphpad Prism 4.01 for Windows (Graphpad Software, San Diego, CA). P-value $\leq 0.05$ was considered statistically significant.

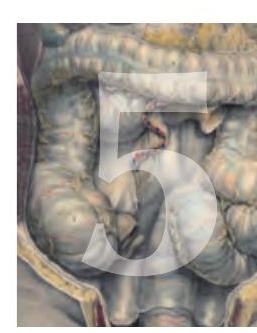




\section{Results}

\section{Patient outcome and reperfusion period}

The mean $( \pm$ SEM) age of included patients $(n=28)$ was 68 (35-79) years and $64 \%$ were male. Isolated jejunum samples were subjected to 30 minutes of ischemia followed by a mean reperfusion time of 123 (30-240) minutes (Fig 1). Jejunum samples subjected to IR were analyzed from 28 patients. Healthy control samples of the jejunum were obtained from 12 patients (43\%), of which we obtained samples with reperfusion times of over 60 minutes.

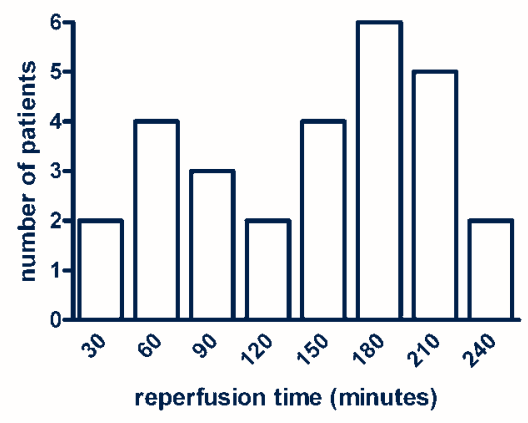

Figure 1: Reperfusion times

Distribution of total reperfusion times following 30 minutes of ischemia $(n=28)$.

\section{Plasma LPS levels following IR}

Disruption of the gut barrier may lead to translocation of microbiota and proinflammatory microbial products, such as the endotoxin associated with gram negative bacteria, lipopolysacharide (LPS). Therefore we assessed the translocation of LPS following the rapid and temporary disruption of the epithelial lining in response to IR observed in our model. ${ }^{1}$ LPS translocation from the intestinal lumen was assessed by measuring arterio-venous (AV) differences in LPS concentration across the studied jejunum, using the sensitive LAL-assay. Surprisingly, no translocation of LPS $(p=0.18 . n=28)$ was detected over the total course of reperfusion (Fig 2). The temporary loss of gut wall integrity observed during reperfusion, which is induced by massive shedding of apoptotic epithelial cells, does not lead to the translocation of highly proinflammatory bacterial product LPS. 


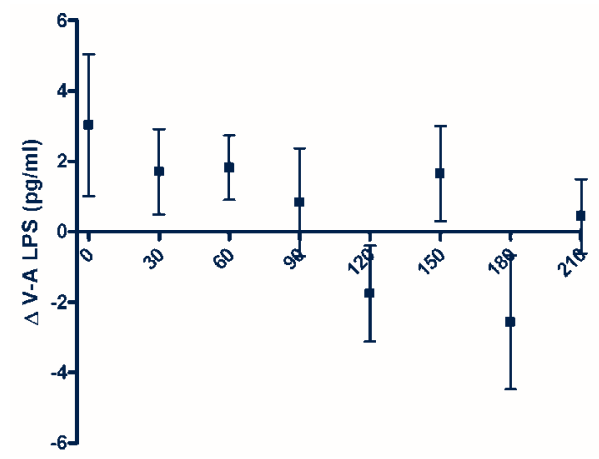

Figure 2: Tissue response to IR

Illustrative of rapid gut barrier repair, no translocation of LPS could be detected during the IR procedure. Translocation of LPS was calculated from arteriovenous differences in LPS plasma levels across the isolated bowel segment during reperfusion $(\Delta(\mathrm{V}-\mathrm{A}) . \mathrm{p}=0.18)$.

\section{Stress response}

In order to demonstrate a physiological response to anoxia and to reperfusion stress, the gene expression profile of hypoxia inducible factor- $1 \alpha(\mathrm{HIF}-1 \alpha)$ and heme oxygenase-1 (HO-1) were determined in healthy and IR damaged whole jejunum samples $(n=12)$. The gene expression of HIF-1 $\alpha$, that regulates both the physiologic and pathophysiologic responses to ischemia, increased during reperfusion (Table 2). In contrast, local synthesis of HO-1 was unaltered by IR (Table 2).

\section{Cytokine expression and endothelial activation}

In order to assess the inflammatory reaction over the course of IR, serial venous blood samples from the reperfused jejunum were analyzed for different inflammatory markers. Additionally, the cytokine response in whole jejunum tissue samples was studied using Q-PCR and endothelial cell activation was assessed by immunohistochemistry on paraffin embedded whole jejunum tissue samples. No increase in TNF $\alpha$ protein levels was detected in venous plasma samples, collected from the isolated jejunum during the procedure; e.g. prior to ischemia, directly after ischemia and every 30 minutes during reperfusion. Compared to the TNF $\alpha$ levels in arterial circulation, AV-differences in TNF $\alpha$ levels revealed no release of TNF $\alpha$ (data not shown). In line, no increase in TNF $\alpha$ mRNA was detected in IR damaged jejunum samples, when compared to healthy jejunum controls from the same patient (Table 2).

Gene expression of cytokines IL-6 (Table 2) and IL-8 (Table 2) in response to IR differed widely and lacked to show an increase in gene expression $(\mathrm{n}=$ 


\section{Chapter 5}

12). Of interest is the observation that the length of reperfusion period in different individuals did not correlate with the IL-6 or IL-8 mRNA levels (data not shown).

Classic in the development of extensive IR damage is the early expression of various adhesion molecules by activated endothelial cells. Presence of E-selectin, ICAM-1 and MADCAM-1 were analyzed using immunohistochemistry. Only 3 patient samples demonstrated sporadic and minimal expression of E-selectin (data not shown), ICAM-1 and MADCAM-1 (Fig 3) following maximal reperfusion.

Table 2: Gene expression profiles in response to IR, calculated by comparative DDCT method of relative quantification. ${ }^{*}$ p-value $\leq 0.05$ was considered statistically significant $(n=12)$

\begin{tabular}{lllll} 
Gene & Healthy & $\mathbb{R}$ & Fold increase $(95 \%-\mathrm{Cl})$ & $\mathrm{P}$ - value \\
\hline HIF-1 $\alpha$ & 1.00 & 1.91 & $1.91(1.15-2.67)$ & $0.02^{*}$ \\
HO-1 & 1.00 & 1.58 & $1.58(0.22-2.94)$ & 0.34 \\
TNF $\alpha$ & 1.00 & 1.18 & $1.18(0.56-1.80)$ & 0.54 \\
IL-6 & 1.00 & 12.31 & $12.31(-5.29-29.91)$ & 0.18 \\
IL-8 & 1.00 & 15.86 & $15.86(0.59-31.13)$ & 0.06 \\
C3 & 1.00 & 4.09 & $4.09(1.74-6.43)$ & $0.01^{*}$ \\
\hline
\end{tabular}

ICAM-1

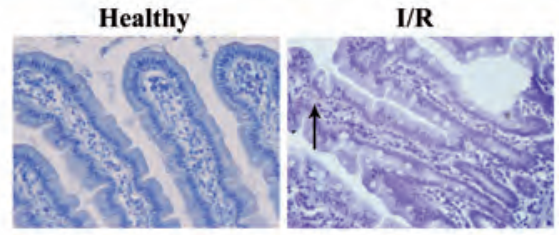

MADCAM-1

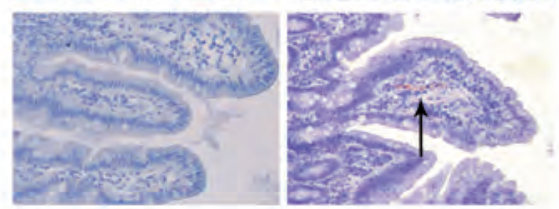

Figure 3: Endothelial activation

Compared to healthy control jejunum tissue, reperfused jejunum samples in response to 30 minutes of ischemia show an only moderate increase in ICAM-1 (upper panel) and MADCAM-1 (lower panel) expression demonstrated by terracotta red staining of the AEC. Original magnification 200x. 


\section{Neutrophil infiltration and release of myeloperoxidase}

Critical in the induction of reperfusion injury is the influx of neutrophils as well as the release of their reactive constituents. The presence of neutrophils was assessed using two different and widely used neutrophil markers, human neutrophil defensin 1-3 (HNP1-3) and myeloperoxidase (MPO), both stored in abundance in azurophilic granules of neutrophils. Microscopic analysis of multiple high power fields (5) from different paraffin embedded tissue samples by a blinded observer, showed that the number of PMN in the reperfused jejunum was similar in comparison to the number of PMN observed in healthy control jejunum samples, as detected by immunohistochemical analysis of HNP1-3 (Fig 4A. $p=0.90 . n=10)$. In concordance, more sensitive analysis of whole jejunum samples for total MPO content by ELISA, revealed no increase in MPO protein in the reperfused jejunum, when compared to healthy jejunum control samples (Fig 4B. $\mathrm{I} / \mathrm{R}=101.0 \pm 31.3 \mathrm{ng} / \mathrm{mg}$ vs Healthy $=75.9 \pm 32.6 \mathrm{ng} / \mathrm{mg} . \mathrm{p}=0.71 . \mathrm{n}=12$ ). A small number of HNP1-3 positive PMN were observed in cellular debris in the intestinal lumen (Fig 4A insert). The presence of these PMN in the intestinal lumen was apparently of no considerable consequence to the remaining jejunum.

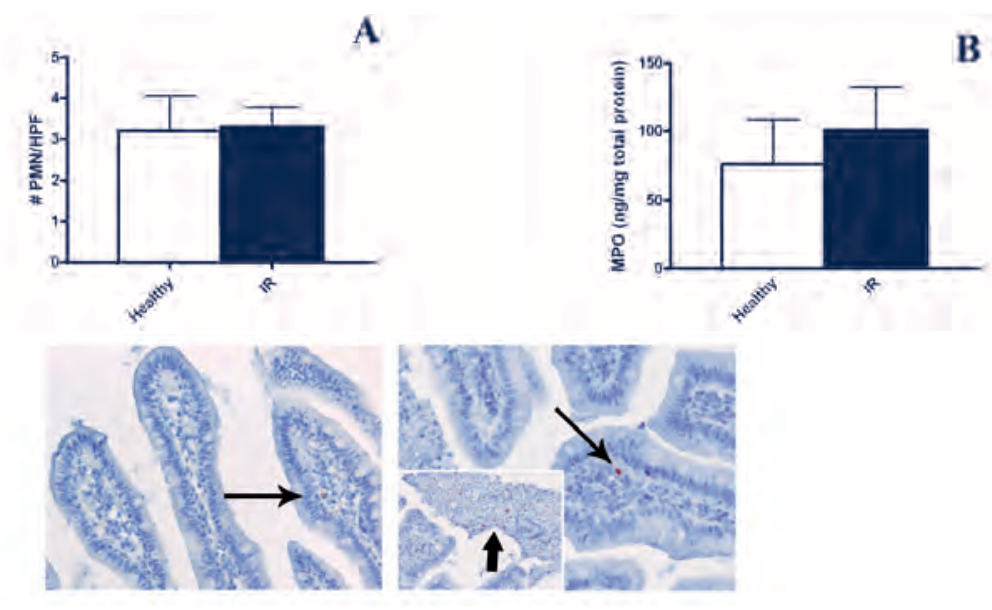

Figure 4: Neutrophil recruitment

A) No increase of PMN was observed over the course of reperfusion in response to 30 minutes of ischemia. Detected by specific HNP1-3 staining (AEC) the number of PMN in reperfused jejunum did not increase in comparison to healthy tissue $(p=0.90)$. Original magnification 200x. Clearly the PMN concentrated around the cellular debris collecting in the safe intestinal lumen (right insert in Fig 4A. Original magnification 200x). B) Tissue MPO, assayed by ELISA, does not increase substantially over the course of IR $(p=0.71)$. 


\section{Chapter 5}

\section{Complement activation and expression in response to IR of the jejunum}

As a central mediator of IR induced inflammation, complement is involved in the development of organ damage. Complement component $\mathrm{C} 3$ is an important constituent of the complement system, of which activation and deposition indicate complement activation. Immunohistochemical analysis of healthy and IR damaged jejunum sections revealed no increase in $\mathrm{C} 3$ deposition in response to IR (Fig 5A). In order to validate these data, human C3 protein in total jejunum tissue after reperfusion was additionally analyzed by western blot. Similar, jejunum tissue specimens showed no increase in the presence of $\mathrm{C} 3$ over the course of IR, when compared to their healthy controls (Fig 5B. I/R $=3.09 \pm 1.79$ relative intensity vs Healthy $=1.00$ relative intensity. $p=0.140 . n=12$ ).

Interestingly, subsequent analysis of the response to IR by the jejunum involving C3 mRNA, using Q-PCR analysis, clearly detected C3 mRNA levels in both healthy and IR damaged whole jejunum samples. During reperfusion, the amount of C3 specific mRNA increased over 4 times in response to 30 minutes of ischemia (Table 2), signifying the tissue's responses to ischemia rather than activation of a vigorous inflammatory reaction in response to tissue damage and by recruitment of plasma complement components.

A

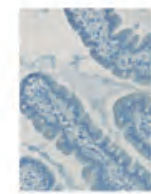

Healthy

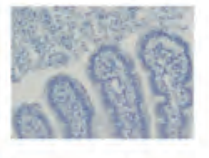

IR

C

B

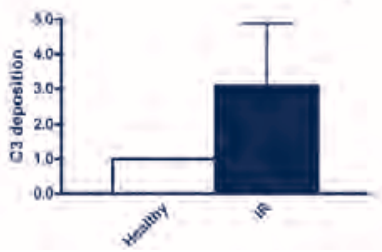

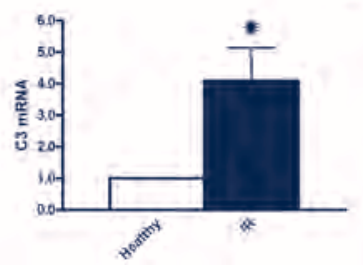

Figure 5: Complement activation

A) C3 was not be detected in the reperfused jejunum using immunohistochemistry. Original magnification 200x. B) Western blot analysis, under reducing conditions, revealed no increase in human C3 (molecular weight $\pm 80 \mathrm{kD}$ ) in whole reperfused jejunum samples, when compared to healthy control tissue $(p=0.14)$. C) C3 gene expression increased over 4-fold during IR of the human jejunum $(p=0.01)$. 


\section{Discussion}

Development of IR induced organ damage is generally characterized by an excessive and vigorous inflammatory response, mainly triggered by massive apoptosis of cells that cannot be resolved in time by phagocytic cells and will be become necrotic in time. Such necrotic cells are a source of damage associated molecular patterns (DAMPS) or alarmins which recruit and activate innate immune cells in a process aimed at restoration of homeostasis by resolving tissue damage and promoting tissue repair. ${ }^{24,25}$ In the context of ischemia and reperfusion such an inflammatory reaction often results in additional tissue damage. It has been demonstrated in the kidney as well as other organs that IR induced inflammation and subsequent organ damage is dependent on the development of widespread apoptosis. ${ }^{4}$ Rapid clearance of apoptotic cells as well as therapeutic strategies to reduce apoptosis have been shown to be critical in preventing the mostly harmful IR induced inflammatory response. ${ }^{2-7,26}$

Critical in the execution of apoptosis is the activation of proteolitic enzymes, called caspases. Caspase-1, also referred to as IL-1-converting enzyme (ICE) is also involved in the cleavage and activation of cytokines, such as IL-1, IL12 and IL-18. ${ }^{27,}{ }^{28}$ Also the cytokine activity of TNF $\alpha$, IL- 6 and IL- 8 is associated with the inflammatory reaction following IR. ${ }^{29}$ As a prototypic member of the TNF-family, TNF- $\alpha$ is a key mediator of acute inflammation of which expression is readily induced following ischemia reperfusion of the rat small intestine. ${ }^{30}$ Interestingly, we did not detect an increase in TNF $\alpha$ message in our experiments. Similarly, using IL-6 knockout (KO) mice, IL-6 was demonstrated as an important component of the acute phase reaction, able to induce tissue injury and inflammation following mesenteric ischemia and reperfusion. ${ }^{31}$ IL-6 controls endothelial cell injury, mediating successive neutrophil influx, although antiinflammatory properties of IL-6 have been reported as well. Recent work on small bowel transplantation in rats demonstrated that exogenous administration of IL-6 to the donor prior to transplantation reduced cold IR injury associated with small bowel transplantation. ${ }^{32}$ Also the chemokine IL-8 has been shown to be involved in intestinal ischemia reperfusion induced inflammation. Administration of an inhibitory anti IL-8 antibody over the course of mesenteric IR in rats prevented neutrophil infiltration and protected the small intestine from IR injury. ${ }^{33}$ In line with our TNF $\alpha$ and IL-6 data also IL-8 gene expression was not up regulated following human intestinal ischemia reperfusion. 


\section{Chapter 5}

E-Selectin (CD-62-E and formerly known as endothelial leucocyte adhesion molecule-1 (ELAM-1)) has an important role in the recruitment of leucocytes to sites of tissue injury. Its expression by vascular endothelial cells in response to injury is readily induced by cytokines IL-1 and TNF $\alpha$. Interestingly, our data suggest that despite the massive presence of dead cells in response to an IR period, rapid shedding of damaged epithelial debris largely averts endothelial cell activation. Consistent with this observation, neutrophil infiltration, assessed by HNP1-3 positive cells in IR and dependant on chemokine release, endothelial activation and chemotaxis by anaphylatoxins, was not detected in our experiments. In line with these data no increase in release of MPO was observed. Although MPO is normally associated with the antimicrobial activity of neutrophils, the presence of MPO has been demonstrated to have a clear effect on the development of IR induced organ damage. Renal IR studies have demonstrated that MPO itself is able to contribute to the development of organ damage. ${ }^{34}$

The complement system is a central innate immune regulator that is activated during intestinal IR and contributes substantially to IR induced inflammation, organ damage and failure. ${ }^{16,17,35}$ Preventing complement activation has been proven to be beneficial to organ function after IR in general and intestinal IR more specifcally. ${ }^{17,18,36}$ Central in the activation of complement is the formation of C3a, an anaphylatoxin with the ability to attract inflammatory cells into the reperfused ischemic tissue. In our model no deposition of C3 in reperfused jejunum was detected, using immunohistochemical and western blot analysis. Interestingly, our results do however suggest $\mathrm{C} 3$ gene expression in healthy jejunum tissue as well as an increased synthesis of $\mathrm{C} 3$ in response to IR. The ability of small intestinal epithelial cells to express biological mediators such as C3 in response to IR was to be expected since previous work demonstrated C3 gene expression in inflamed small intestine during Crohn's disease. ${ }^{37}$ To the best of our knowledge, however, these are the first results that demonstrate $\mathrm{C} 3$ synthesis in the healthy jejunum. Further analysis will have to elucidate the nature increased C3 expression following IR.

The recently discovered molecular mechanism that recognizes and responds to excessive cell death consisting of SAP130 and the macrophage inducible C-type lectin (Mincle) fits in our observations. ${ }^{38}$ The loss of excessive numbers of dead cells into the gut lumen prevents SAP130 released by the dead cells to reach tissue macrophages thus largely preventing the production of inflammatory cytokines driving rapid neutrophil infiltration. Similarly activation of complement by dead cells is prevented therefore the PMN chemotactic factors C3a en C5a will not be produced. 
Another mechanism of protection was suggested by the enhanced expression of HIF- $1 \alpha$ in the surviving epithelium. Expression of HIF- $1 \alpha$ in response to low oxygen tension during ischemia and early reperfusion triggers physiologic responses characterized by activation of functional proteins mucin, P-glycoprotein, intestinal trefoil factor and adenosine $\mathrm{A} 2 \mathrm{~B}$ receptor, aimed at preventing mucosal inflammation. ${ }^{39}$ The role in preserving gut wall integrity in response to inflammation as well as the exciting role of HIF- $1 \alpha$ in different cardiac and brain pre-conditioning IR models underlines the protective influence of increased in HIF-1 $\alpha$ gene expression as detected in our model in response to IR of the human intestine. $^{39-41}$

Ischemia, sensed by the small intestine, induces normal physiological and IR induced responses. Our data provide new and compelling evidence of increased HIF-1 $\alpha$ and also C3 gene expression during the reperfusion period. However, these responses are not paralleled by an IR induced inflammatory response, since important conditions to an inflammatory reaction have not been met by the absence of dead cells in the reperfused tissue. It is important to realize that these data indicate that the human intestine is more resistant to IR than initially thought. Its ability to shed damaged epithelial cells and repair its ever important barrier function without triggering massive inflammation can be seen as one of the key features that prevents the gut from inflammation following splanchnic hypoperfusion as occurs in situations with circulatory redistribution in favour of the brain and crucial organs. Moreover this specific property of the gut enables surgical interventions such as prolonged mesenteric clamping.

Taken together, our data suggest that the ability of the small intestine to shed cells damaged by ischemia and reperfusion into the exterior environment of the intestinal lumen protects the surviving tissue from the exposition to highly pro-inflammatory DAMPS or alarmins originating from dead cells. A new physiological rescue mechanism is revealed by which acute pro-inflammatory stimuli are safely eliminated and discarded into the intestinal lumen following moderate ischemia of the small gut, explaining the absence of a cytokine response and neutrophil recruitment. Other organs might well exhibit similar responses with limiting success. The kidney for example discards its damaged tubular epithelial cells into its tubular lumen in response to IR, acute tubular necrosis or toxic ingestion. The limited space in the tubules might restrict the effect of this process. 


\section{Chapter 5}

\section{ReferenCes}

1. Derikx JPM, Matthijsen RA, de Bruïne AP, et al. Rapid Reversal of Human Intestinal Ischemia-Reperfusion Induced Damage by Shedding of Injured Enterocytes and Reepithelialisation. PLOS ONE 2008;3:e3428.

2. Yellon DM, Hausenloy DJ. Myocardial reperfusion injury. N Engl J Med 2007;357:1121-35.

3. de Groot $\mathrm{H}$, Rauen U. Ischemia-reperfusion injury: processes in pathogenetic networks: a review. Transplant Proc 2007;39:481-4.

4. Daemen MA, van 't Veer C, Denecker G, et al. Inhibition of apoptosis induced by ischemia-reperfusion prevents inflammation. J Clin Invest 1999;104:541-9.

5. Donath S, Li P, Willenbockel C, Al-Saadi N, et al. Apoptosis repressor with caspase recruitment domain is required for cardioprotection in response to biomechanical and ischemic stress. Circulation 2006;113:1203-12.

6. Yaoita H, Ogawa K, Maehara K, et al. Attenuation of ischemia/reperfusion injury in rats by a caspase inhibitor. Circulation 1998;97:276-81.

7. Restifo NP. Building better vaccines: how apoptotic cell death can induce inflammation and activate innate and adaptive immunity. Curr Opin Immunol 2000;12:597-603.

8. Litt MR, Jeremy RW, Weisman HF, et al. Neutrophil depletion limited to reperfusion reduces myocardial infarct size after 90 minutes of ischemia. Evidence for neutrophilmediated reperfusion injury. Circulation 1989;80:1816-27.

9. Daemen MA, van de Ven MW, Heineman E, et al. Involvement of endogenous interleukin-10 and tumor necrosis factor-alpha in renal ischemia-reperfusion injury. Transplantation 1999;67:792-800.

10. Frangogiannis NG, Smith CW, Entman ML. The inflammatory response in myocardial infarction. Cardiovasc Res 2002;53:31-47.

11. Takada M, Nadeau KC, Shaw GD, et al. The cytokine-adhesion molecule cascade in ischemia/reperfusion injury of the rat kidney. Inhibition by a soluble P-selectin ligand. J Clin Invest 1997;99:2682-90.

12. Romson JL, Hook BG, Kunkel SL, et al. Reduction of the extent of ischemic myocardial injury by neutrophil depletion in the dog. Circulation 1983;67:1016-23.

13. Lucchesi BR. Modulation of leukocyte-mediated myocardial reperfusion injury. Annu Rev Physiol 1990;52:561-76.

14. de Vries B, Kohl J, Leclercq WK, et al. Complement factor C5a mediates renal ischemiareperfusion injury independent from neutrophils. J Immunol 2003;170:3883-9.

15. Guo RF, Ward PA. Role of C5a in inflammatory responses. Annu Rev Immunol 2005;23:821-52.

16. Zhou W, Farrar CA, Abe K, et al. Predominant role for C5b-9 in renal ischemia/reperfusion injury. J Clin Invest 2000;105:1363-71. 
17. Hart ML, Ceonzo KA, Shaffer LA, et al. Gastrointestinal ischemia-reperfusion injury is lectin complement pathway dependent without involving C1q. I Immunol 2005; 174:6373-80.

18. De Vries B, Matthijsen RA, Wolfs TG, et al. Inhibition of complement factor C5 protects against renal ischemia-reperfusion injury: inhibition of late apoptosis and inflammation. Transplantation 2003;75:375-82.

19. Murohara T, Delyani JA, Albelda SM, et al. Blockade of platelet endothelial cell adhesion molecule-1 protects against myocardial ischemia and reperfusion injury in cats. J Immunol 1996;156:3550-7.

20. Schoenberg MH, Poch B, Younes M, et al. Involvement of neutrophils in postischaemic damage to the small intestine. Gut 1991;32:905-12.

21. Ma XL, Tsao PS, Lefer AM. Antibody to CD-18 exerts endothelial and cardiac protective effects in myocardial ischemia and reperfusion. J Clin Invest 1991;88:1237-43.

22. Kanda T, Fujii H, Tani T, et al. Intestinal fatty acid-binding protein is a useful diagnostic marker for mesenteric infarction in humans. Gastroenterology 1996;110:339-43.

23. Engelberts I, Stephens S, Francot GJ, et al. Evidence for different effects of soluble TNFreceptors on various TNF measurements in human biological fluids. Lancet 1991;338:515-6.

24. Bianchi ME. DAMPs, PAMPs and alarmins: all we need to know about danger. J Leukoc Biol 2007;81:1-5.

25. Lotze MT, Zeh HJ, Rubartelli A, et al. The grateful dead: damage-associated molecular pattern molecules and reduction/oxidation regulate immunity. Immunol Rev 2007;220:60-81.

26. Huynh ML, Fadok VA, Henson PM. Phosphatidylserine-dependent ingestion of apoptotic cells promotes TGF-beta1 secretion and the resolution of inflammation. J Clin Invest 2002;109:41-50.

27. Daemen MA, van't Veer C, Wolfs TG, et al. Ischemia/reperfusion-induced IFN-gamma up-regulation: involvement of IL-12 and IL-18. J Immunol 1999;162:5506-10.

28. Miwa K, Asano M, Horai R, et al. Caspase 1-independent IL-1beta release and inflammation induced by the apoptosis inducer Fas ligand. Nat Med 1998;4:1287-92.

29. Grotz MR, Deitch EA, Ding J, et al. Intestinal cytokine response after gut ischemia: role of gut barrier failure. Ann Surg 1999;229:478-86.

30. Salvemini D, Wang ZQ, Zweier JL, et al. A nonpeptidyl mimic of superoxide dismutase with therapeutic activity in rats. Science 1999;286:304-6.

31. Cuzzocrea S, De Sarro G, Costantino G, et al. IL-6 knock-out mice exhibit resistance to splanchnic artery occlusion shock. J Leukoc Biol 1999;66:471-80.

32. Kimizuka K, Nakao A, Nalesnik MA, et al. Exogenous IL-6 inhibits acute inflammatory responses and prevents ischemia/reperfusion injury after intestinal transplantation. Am J Transplant 2004;4:482-94. 


\section{Chapter 5}

33. Tsuruma T, Yagihashi A, Tarumi K, et al. Anti-rat IL-8 (CINC) monoclonal antibody administration reduces ischemia-reperfusion injury in small intestine. Transplant Proc 1998;30:2644-5.

34. Matthijsen RA, Huugen D, Hoebers NT, et al. Myeloperoxidase is critically involved in the induction of organ damage after renal ischemia reperfusion. Am / Pathol 2007; 171:1743-52.

35. Arumugam TV, Shiels IA, Woodruff TM, et al. The role of the complement system in ischemia-reperfusion injury. Shock 2004;21:401-9.

36. Wada K, Montalto MC, Stahl GL. Inhibition of complement C5 reduces local and remote organ injury after intestinal ischemia/reperfusion in the rat. Gastroenterology 2001;120:126-33.

37. Laufer J, Oren R, Goldberg I, et al. Cellular localization of complement C3 and C4 transcripts in intestinal specimens from patients with Crohn's disease. Clin Exp Immunol 2000;120:30-7.

38. Yamasaki S, Ishikawa E, Sakuma M, et al. Mincle is an ITAM-coupled activating receptor that senses damaged cells. Nat Immunol 2008;9:1179-88.

39. Taylor CT, Colgan SP. Hypoxia and gastrointestinal disease. J Mol Med 2007;85:1295300.

40. Bergeron $M$, Gidday JM, Yu AY, et al. Role of hypoxia-inducible factor-1 in hypoxiainduced ischemic tolerance in neonatal rat brain. Ann Neurol 2000;48:285-96.

41. Cai Z, Manalo DJ, Wei G, et al. Hearts from rodents exposed to intermittent hypoxia or erythropoietin are protected against ischemia-reperfusion injury. Circulation 2003;108:79-85. 
Figg, 5

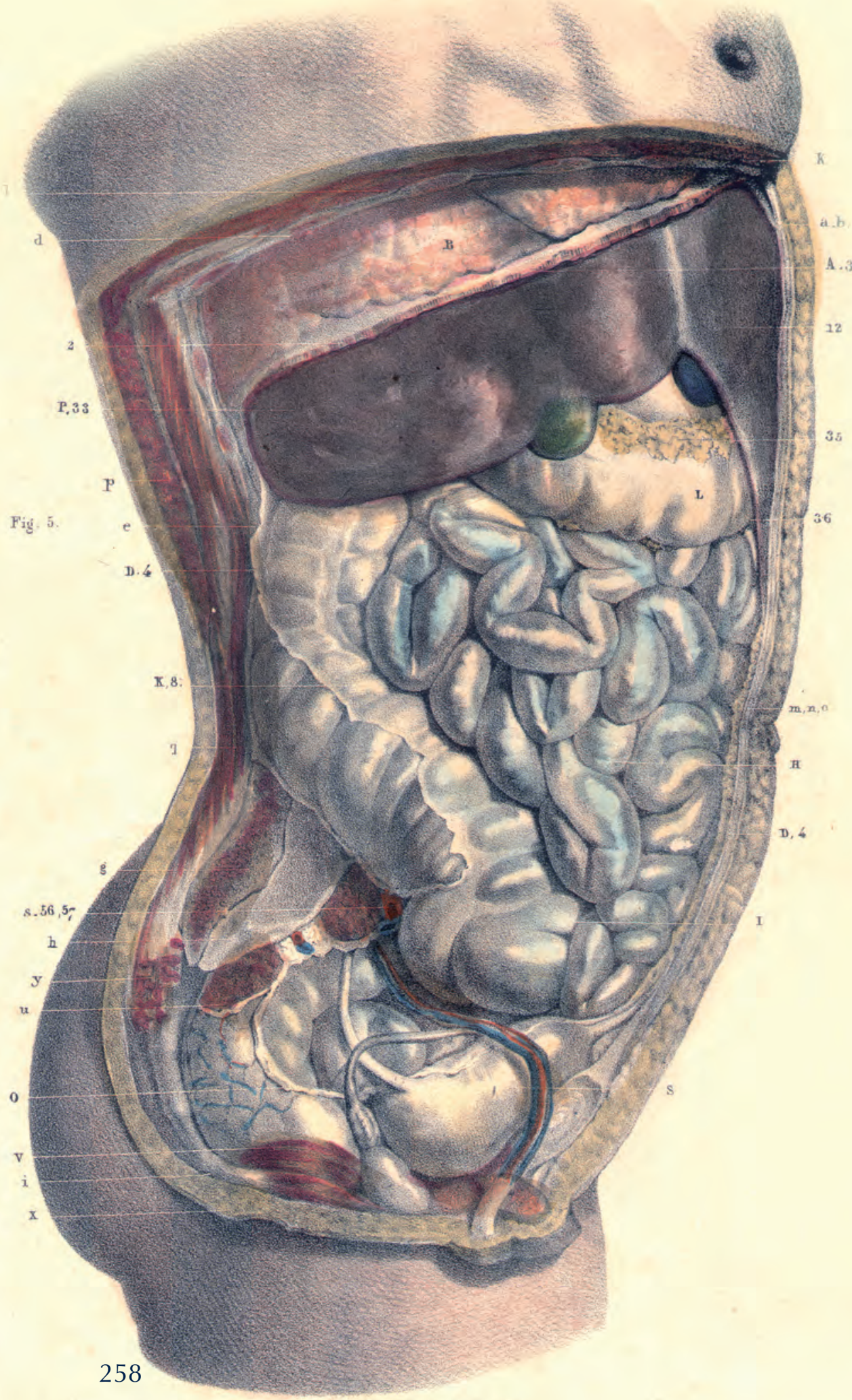




\section{Chapter 6}

\section{Summary and discussion}


Chapter 6 
The intestinal mucosa is responsible for the absorption of nutrients from the lumen on the one hand and for the separation of the potentially toxic luminal content (external environment) from the host (internal environment) on the other hand. Disruption of this delicate balance at the mucosal interface occurs in numerous (intestinal) diseases. Assessment of gut wall integrity loss in clinical practice is still a challenge, since it is difficult to evaluate gut condition non-invasively with the currently available diagnostic tools. Moreover, noninvasive, rapid diagnostic means to assess intestinal condition are also needed in the follow-up of many intestinal diseases for evaluation of the effects of treatment on the recovery of the disorder. Furthermore, primarily experimental animal studies have shown that gut wall integrity loss is involved in the development of various inflammatory syndromes, including post-operative or post-traumatic systemic inflammatory response syndrome (SIRS), sepsis and multiple organ failure (MOF) ${ }^{1,2}$.

In this thesis, markers for human gut wall integrity were evaluated as tools for diagnosis and follow-up in patient studies. Next, the development of gut wall integrity loss in critically ill patients and patients undergoing major surgery was investigated to study whether gut barrier loss is of importance in the development of post-operative complications. Finally, as intestinal ischemiareperfusion was proven to be present in critically ill patients and in patients undergoing major surgery and was related to the onset of gut wall integrity loss, the sequelae of intestinal ischemia-reperfusion were investigated in a novel human model.

The first aim of this thesis was to gain more insight in characteristics of markers that are potentially useful for the assessment of gut wall integrity loss, namely tests for enterocyte damage and tight junction loss.

This thesis focussed on markers for enterocyte damage, more specifically on the family of fatty acid binding proteins (FABP), since these proteins are tissue specific ${ }^{3}$. Three isoforms of FABP are present in the intestine: intestinal (I)-FABP, liver (L)-FABP, ileal-bile acid binding protein (I-BABP) ${ }^{3}$. Results of previous experimental animal studies and some human (pilot-)studies have shown that FABP are potentially useful as plasma- and/or urinary markers to detect enterocyte damage, as occurs after ischaemia or inflammation ${ }^{4,5}$. To gain insight in FABP plasma and urinary concentrations, the exact localization of these proteins in the gut was investigated. The expression of I-FABP and LFABP in the different segments of the human intestine, obtained during routine surgery, was evaluated (in the second paragraph of chapter 2). I-FABP and LFABP are expressed in the mucosa of the entire small intestine and have a 


\section{Chapter 6}

predominant expression in the jejunum. I-BABP is specifically present in the ileum with a mean $( \pm$ SEM) concentration of $303( \pm 113) \mu \mathrm{g}$ I-BABP/gr tissue (data not shown). I-FABP, L-FABP and I-BABP are in particular highly expressed in cells present on the top of the villi. The presence of FABP on the top of the villi, the initial site of destruction in numerous intestinal diseases, makes circulating FABP potentially useful plasma markers in early stages of intestinal diseases. Subsequently, the release of I-FABP and L-FABP into the circulation of patients with obvious intestinal epithelial damage, i.e. patients with celiac disease, was investigated. This pilot-study showed that I- and L-FABP circulating levels were significantly increased in untreated celiac disease compared to healthy volunteers. Furthermore, I- and L-FABP concentration normalized after implementation of the therapy for celiac disease (gluten-free diet) in all but one patient. However, one has to bear in mind that L-FABP shows a multi-tissue expression, in contrast to I-FABP. Besides the expression in the intestine, L-FABP is also present in hepatocytes and tubular cells of the kidney ${ }^{3}$. Increased circulating and/or urinary L-FABP levels can therefore be derived from other organs than the intestine.

Subsequently, the release and clearance of FABP was investigated in patients undergoing liver resection for secondary tumors in an otherwise healthy liver (as is described in paragraph 3, chapter 2). Plasma levels of L-FABP increased early during surgery, namely during liver manipulation. Other classical liver injury markers (alpha glutathione s-transferase (GSTa) and aspartate amino transferase (AST) showed a similar rise. L-FABP and GSTa levels decreased immediately postoperatively, whereas AST gradually kept increasing. As a consequence, L-FABP and GSTa are probably more sensitive for detecting ongoing hepatocyte injury and impending liver failure than AST. To prove this assumption, however, a large prospective study is needed.

Next, the release and clearance of FABP was investigated in the same study by collection of blood from efferent and afferent vessels of the intestine, liver and kidney during surgery. Arterial renal venous concentration gradients showed that the kidneys remove approximately $30 \%$ of L-FABP in a single pass, leading to a calculated L-FABP half-life of 11 minutes. In conclusion, the immediate postoperative decline of L-FABP is a result of its rapid renal clearance. This further emphasizes that FABP is an accurate marker for actual cell damage and that urinary concentration are potentially useful in reflecting this damage, since FABP are rapidly cleared by the kidneys.

As the epithelial barrier is constituted by both enterocytes and tight junctions, the next study was directed at finding a marker for the assessment of tight junction damage. In chapter 2, paragraph 4 was studied whether an 
important tight junction protein, i.e. claudin- $3^{6}$, can be used as a non-invasive marker to study the condition of tight junctions. In a translational study, using both a rat hemorrhagic shock model and a human setting of patients with active inflammatory bowel disease (IBD), the immunohistochemically visualized loss of claudin-3 from intestinal tissue resulted quickly in the appearance of this protein into urine. This is the first study to report that measurement of the status of tight junctions can be performed non-invasively.

In conclusion, the results of chapter 2 showed that non-invasive (i.e. urine based) assessment of the condition of the epithelial layer of the gut, which is responsible for the barrier between external environment and host, is possible with the use of proteins originating form cytoplasma of damaged enterocytes (FABP) and from disrupted tight junctions (claudin-3) (Figure 1).

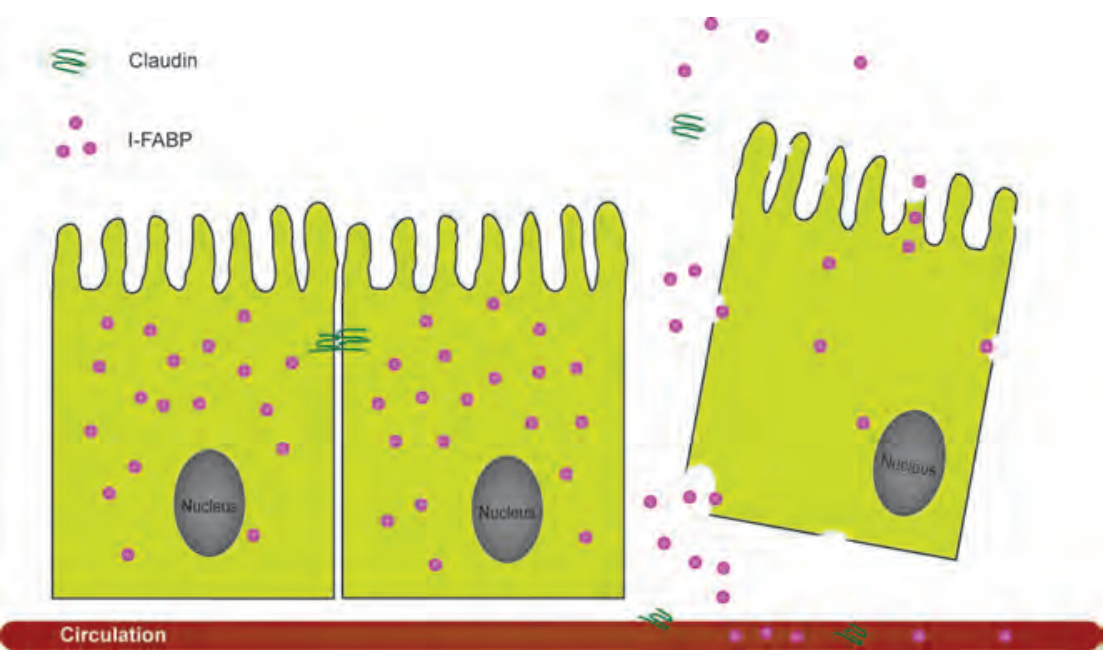

Figure 1: the leakage of FABP and claudin, reflecting the condition of the epithelial layer of the gut.

The second aim of this thesis was to analyse the usefulness of markers for compromised gut wall in patients with overt gut damage and in patients with suspected intestinal disease to distinguish the target disease from other pathologies.

Diagnostic tests aim at discriminating between clinically "normal" and "abnormal". New diagnostic tests should subsequently answer the following 4 questions before implementation of the new test in daily clinical practice can be contemplated ${ }^{7}$. 


\section{Chapter 6}

Question 1: Do patients with the target disorder have different test results from normal individuals? The answer requires comparison of test results among individuals with the disease and individuals that are disease-free. In chapter 3, paragraph 2, a 2-year-old girl with rhabdomyosarcoma is presented, who suffered from three episodes of gastrointestinal mucositis and bacteraemia following two cytostatic treatments consisting of ifosfamide, vincristine, and actinomycin D (IVA). This is a well-known and potentially harmful complication of chemotherapy, which is difficult to diagnose and of which the pathophysiology is poorly understood. Gastrointestinal mucositis presented as abdominal complaints, fever, which was accompanied by raised C-reactive protein levels, and positive blood culture for coagulase-negative staphylococci. The signs and symptoms and laboratory results were preceded by increased plasma levels of intestinal fatty acid binding protein (I-FABP), reflecting enterocyte damage. Next, the relationship between enterocyte mass and enterocyte loss was explored by examining citrulline serum levels (reflecting functional enterocyte mass) ${ }^{8,9}$ and by assessing circulating I-FABP and I-BABP (assessing enterocyte loss) in adult patients with haematological malignancy receiving allogeneic HSCT and preceding myeloablative conditioning, consisting of idarubicin, cyclophosphamide and total body irradiation (chapter 3, paragraph 3). This regimen is known to induce oral and intestinal mucosal barrier injury ${ }^{10}$. A decline in circulating citrulline concentrations was found with the nadir 19 days after the start of the myeloablative regimen. This is in line with previous studies and shows a clear transient reduction in the small intestinal epithelial cell mass. Interestingly, an almost simultaneous reduction in circulating levels of I-FABP and I-BABP were observed. The baseline circulating levels of FABP are considered to represent the continuous loss or, in other words, the turnover of enterocytes, since the main loss of enterocytes in the healthy intestine consists of senescent enterocytes which release FABP upon detachment or death into the gut lumen at the end of their lifespan. Surprisingly, these data showed that less cytosolic content of enterocytes is released into the circulation after intensive myeloablative conditioning than under normal, healthy circumstances, indicating a decreased rate of dying enterocytes. This, in turn, suggests a reduced turnover of enterocytes after myeloablative conditioning. Furthermore, this is the first study to report on specific changes in I-BABP levels, representing specific jejunal and ileal enterocyte loss, using a newly developed ELISA for measurement of serum and plasma I-BABP.

Question 2: Are patients with certain test results more likely to have the target disorder than patients with other test results? This question is also answered by comparison of test results among patients with the targetdisease and healthy 
individuals, but now test characteristics as sensitivity, specificity and likelihood ratios are estimated. As discussed above in this chapter, the potential usefulness of assessing circulating concentrations of FABP in a pilot study of patients with celiac disease, which is characterized by intestinal mucosal damage, was reported (chapter 2, paragraph 2). The results of the study suggest that besides a possible additional role for FABP in the diagnosis of celiac disease, assessment of plasma/urinary concentration of FABP may provide a new important non-invasive tool to monitor the short-term response to a gluten-free diet and in this way contribute importantly to the follow-up of celiac patients. However, further studies are necessary to determine whether assessment of intestinal epithelial cell damage by FABP analysis can replace the invasive procedure of endoscopy and mucosa biopsy. Next, the usefulness of plasma markers for neutrophil activation products was explored, since gut damage irrevocably leads to gut wall inflammation, which implies the recruitment of leukocytes into the intestinal wall. Acute appendicitis (AA) is an intestinal disease, characterized by gut damage and massive infiltration of neutrophils into the gut wall, for which the diagnosis is often delayed due to its aspecific clinical presentation and lack of adequate laboratory markers. The circulating neutrophil activation product calprotectin showed the most promising results; it was found to be significantly increased in 51 patients with proven AA compared to 9 patients without proven AA and 27 healthy volunteers (chapter 3, paragraph 4). This yielded an overall accuracy of the test, summarized using area under the receiver operating characteristics curve (AUC), of 0.91. This is considered to be clinical relevant.

Question 3: Among patients in whom it is clinically sensible to suspect the target disorder, does the test result distinguish those with and without target disorder? To obtain the appropriate answer, a consecutive series of such patients should be studied. This phase in the evaluation of diagnostic tests was applied to the potential improvement of the diagnostic accuracy of necrotizing enterocolitis (NEC), a serious intestinal neonatal disease with high morbidity and mortality rates, as is reflected in chapter 3, paragraph 6 and 7 . In urine and faeces of 29 consecutively included neonates suspected for NEC proteins representing important (histo-)pathological factors of NEC were measured: epithelial integrity loss (urinary I-FABP and urinary claudin-3) and gut wall inflammation (faecal calprotectin). In the 12 neonates suspected for NEC, who ultimately developed NEC, the levels of the studied markers were significantly higher than in the neonates who finally had other diagnoses (mainly sepsis). Areas under the receiver operating characteristic curve of urinary I-FABP, urinary claudin-3 and faecal calprotectin were 1.0, 0.84 and 0.94 , respectively. These data are considered to be clinically relevant. 


\section{Chapter 6}

Question 4: Do patients who undergo the diagnostic test fare better (in their ultimate health outcomes) than similar patients who do not? The question has to be answered by randomizing patients to undergo the test of interest or another (or no) test. However, it is disputable whether unravelling phase 4 questions is valid and efficient with this design (randomised comparison of the test OR: randomised assignment of patients to the test), since this study design evaluates both the test and different treatment strategies following the tests ${ }^{11}$. Furthermore, a large number of patients is needed. Therefore, an alternative study design for phase 4 questions is to move the point of randomisation from the decision point whether or not to test, to what to do with the test result, i.e. to apply the test to all patients before randomisation, irrespective of the result ${ }^{11}$. If the costs of the test are low compared with the costs of following up patients and monitoring outcome, randomising only test-negative patients potentially offers a more efficient design. Moreover, if the trial is sufficiently powered, assessment of the effectiveness separately in test-positive and test-negative patients can lead to evidence-based practice recommendations.

In conclusion, the potential usefulness of new markers (FABP, claudin-3, calprotectin) in the early diagnosis of intestinal diseases was shown. It is of importance that most of the tests can be performed in non-invasively collected material, such as urine and faeces. Especially in neonates and children this is a great advantage, since blood collection for diagnostic purposes is traumatic for all children and a major cause for anaemia in neonates. All studies have to be continued into the following phase of the evaluation of new diagnostic tests. For the neonates suspected for NEC, question 4 has to be answered for which the considerations regarding the design have been described.

The third aim of this thesis was to evaluate the development of gut wall integrity loss in critically ill patients and in people undergoing major (nonabdominal) surgery.

Experimental animal studies have generated the hypothesis that the intestines are central in the origin of post-operative and post-traumatic sequelae $1,2,12-14$. It is hypothesized that major surgery or trauma leads to a redistribution of blood to the vital organs (brain, heart) at the expense of the intestines. Especially the mature intestinal mucosal cells are susceptible to hypoperfusion and ischemia. Damage of intestinal mucosa can lead to local inflammation, impaired barrier function, and thereupon potentially to translocation of microorganisms ${ }^{1,2}$. Moreover, cell injury leads to the release of immunostimulatory proteins or nucleotides, so-called danger signals that activate the immune system and induce systemic inflammation ${ }^{15}$. The translocation can result in a 
derailment of local inflammation and subsequently amplification of a systemic inflammatory response, as is mainly shown in animal studies. With the use of the markers for gut wall integrity, which were characterized in previous studies, the involvement of the gut in patients undergoing major surgery was investigated. This is of utmost importance as different opinions exist. First, the development of intestinal hypoperfusion, objectivated by gastric mucosal $\mathrm{PiCO}_{2}$, was explored and its relationship with intestinal epithelial cell damage, assessed by circulating levels of I-FABP, in patients admitted to the intensive care unit (ICU) because of postoperative abdominal sepsis (chapter 4, paragraph 2). Splanchnic hypoperfusion in the early phase of abdominal sepsis correlated strongly with intestinal mucosal damage. Moreover, elevated plasma I-FABP values on admission to the ICU were associated with a poor outcome in patients with abdominal sepsis. Next, in children with meningococcal sepsis it was shown in chapter 4, paragraph 3 that half of the patients presented with intestinal epithelial cell damage at admission to the paediatric ICU and that the children who died were characterized by continued presence of gut damage, while in all survivors this injury came to an end within 12 hours after starting intensive treatment.

In addition to these two clinical retrospective studies showing intestinal epithelial cell damage in a large number of patients immediately upon admission to the ICU, the development of gut wall integrity loss during surgery in two clinical prospective studies was investigated (chapter 4, paragraph 4 and 5).

Two of the most extensive and complex surgical procedures are open surgical repair of thoracic aortic aneurysms (TAA) and thoracoabdominal aortic aneurysms (TAAA) that are associated with significant postoperative morbidity and mortality. In order to prevent peroperative ischemic injury of visceral organs, extracorporeal circulation (ECC) technique has been implemented in open TAA repair to provide (retrograde) distal aortic perfusion (DAP) via the femoral artery. In case of TAAA repair involving the origin of visceral arteries, ECC with DAP is combined with selective organ perfusion catheters (DAP and SP) to provide designated visceral arteries directly with blood. In chapter 4, paragraph 4 it was shown that intestinal mucosal cell injury, but not hepatic or renal tubular cell injury, occurred in patients undergoing elective open TAA $(n=8)$ or TAAA $(n=22)$ repair, despite artificial visceral perfusion. The extent of intestinal injury and following pro-inflammatory reaction was more pronounced in patients undergoing DAP and SP. The most likely explanation for the difference in intestinal injury is a more prominent hypoperfusion of the intestines during ECC with DAP and SP. Furthermore, intestinal injury correlated positively with markers of systemic inflammation (IL-6 and IL-8). Next, the development 


\section{Chapter 6}

of gut wall integrity loss in patients undergoing major spinal fusion surgery was studied, which is characterized by long duration of surgical procedure, significant blood loss, prolonged systemic hypotension and the potential development of postoperative complications (chapter 4, paragraph 5). This type of surgery was chosen because it does not directly compromise the intestines by intestinal manipulation or the use of extracorporeal circulation. In a significant number of patients increased circulating levels of I-FABP and I-BABP and elevated urinary values of claudin-3 were observed, indicating the development of gut wall integrity loss. Furthermore, a significant inverse association between mean arterial pressure and succeeding plasma levels of I-FABP and I-BABP was found, which indicates that systemic hypotension is correlated with intestinal mucosal cell injury, detected $1 / 2$ hour later. The systemic hypotension is mainly caused by anaesthetics, causing a decreased systemic vascular resistance. Finally, splanchnic hypoperfusion (gastric mucosal $\mathrm{PiCO}_{2}$ and $\mathrm{P}_{\mathrm{r}-\mathrm{a}} \mathrm{CO} 2$-gap) correlated strongly with intestinal mucosal damage (plasma I-FABP) at all observed time-points during surgery.

In conclusion, the results of these studies in patients undergoing TAA(A)repair and spinal fusion repair show for the first time the relation between altered splanchnic perfusion and the development of intestinal mucosal cellular damage in patients undergoing major surgery. Collectively, these findings shed a new light on the potential role of intestinal barrier compromise during major surgery, which was deduced from numerous animal studies, but now for the first time reported in relatively healthy children and adolescents undergoing major (non-abdominal) surgery. Furthermore, these results indicate a need to re-examine currently accepted criteria of haemodynamic parameters, both regarding the use of ECC and accepted systemic hypotension, in patients undergoing major surgery.

True, the presence of intestinal damage does not show any cause-andeffect relationship with the development of sepsis or multiple organ failure (MOF). Moreover, intestinal damage may be part of more generalized tissue damage with epithelial barrier dysfunction in lung, liver and kidney ${ }^{16,17}$. These studies are however the basis for further research to clarify the onset of intestinal damage, since direct measurement of intestinal damage has shown interesting results. In addition, assessment of intestinal epithelial damage in patients with sepsis or undergoing major surgery may have important clinical implications. Evaluation of intestinal tissue damage in the early phase of sepsis is an adequate predictor for survival. Furthermore, the adequacy of treatment of circulatory failure in sepsis is currently monitored using indirect parameters of tissue perfusion and oxygenation. However, these parameters do not reflect the 
actual defects in (peripheral) tissue perfusion and subsequent tissue damage. Assessment of plasma I-FABP levels offers the possibility to monitor the presence of intestinal epithelial cell damage as a consequence of splanchnic hypoperfusion, which could help to monitor treatment directed at restoration of peripheral perfusion and prevention of organ damage. Further studies are needed to clarify the diagnostic potential of assessment of plasma I-FABP in monitoring the treatment of sepsis in the acute phase and during follow-up.

As reported in the chapter 4, altered splanchnic perfusion was observed during major surgery and in critically ill patients, which was related to the development of gut wall integrity loss. Therefore, the final aim of this thesis was to set up a model, which enabled us to study the pathophysiological consequences of controlled intestinal ischemia-reperfusion (IR) in detail in man. Moreover, acute mesenteric ischemia is a disease entity itself, which is accompanied by high morbidity and mortality rates because of its aspecific presentation and the lack of accurate markers. A human model for intestinal IR potentially would also give the opportunity to study new diagnostic tests. A pancreatico-duodenectomy is a procedure in which a variable length of jejunum is removed. This enabled us to study IR induced cell damage in a harmless human jejunal IR model. Isolated jejunum $(6 \mathrm{~cm})$ was subjected to 30 minutes ischemia followed by reperfusion, which is described in chapter 5 , paragraph 2.

Mean (SEM) arteriovenous concentration gradients of I-FABP across the jejunum revealed rapid epithelial cell damage, which was dependent on the mannose-binding lectin (MBL) genotype of the patient (chapter 5, paragraph 3 and 4). Overall, I-FABP release significantly increased from $290(46) \mathrm{pg} / \mathrm{ml}$ before ischemia towards 3997 (554) pg/ml immediately after ischemia $(\mathrm{p}<0.001)$ and declined gradually to 1143 (237) $\mathrm{pg} / \mathrm{ml}$ within 1 hour reperfusion $(p<0.001)$. However, in patients with $\mathrm{MBL}$ null alleles no mucosal cell damage, assessed by arteriovenous concentration gradients of I-FABP, was observed immediately after 30 minutes of ischemia, while the highest increase of I-FABP was found in the MBL homozygous wildtype individuals. Directly after ischemia the intestinal epithelial lining was microscopically normal in all patients, while subepithelial spaces appeared at the villus top, caused by contraction of the basement membrane, which demarcates epithelium from lamina propria. However, after 25 minutes reperfusion, apoptosis, visualized by M30 immunostaining, was observed at the villus top accompanied by shedding of mature epithelial cells into the lumen and loss of I-FABP staining of epithelial cells, indicating membrane integrity loss. Interestingly, within 60 minutes 


\section{Chapter 6}

reperfusion the epithelial barrier resealed, while debris of apoptotic, shedded epithelial cells was observed in the lumen. At the same time, both apoptosis and inflammation were absent in intact epithelium (chapter 5, paragraph 5).

In conclusion, this is the first human study to clarify jejunal IR induced cell damage and repair and its direct consequences. It reveals a unique, endogenous clearing mechanism for injured enterocytes upon 30 minutes of ischemia: rapid detachment of damaged apoptotic enterocytes into the lumen. This process is quickly followed by repair of the epithelial continuity, resulting in a normal epithelial lining, which is hypothesized to be a key factor in the prevention of the development of inflammation. Our results might explain why the gut can tolerate 30 minutes of ischemia in events as exercise, trauma, sepsis, repair of a (thoraco-)abdominal aneurysm of the aorta, and cardiopulmonary bypass. It is of importance to understand the maximal time of ischemia, which the gut can bear by utilization of its repair mechanism for injured cells.

\section{REFERENCES}

1. Moore FA. The role of the gastrointestinal tract in postinjury multiple organ failure. Am J Surg 1999;178(6):449-53.

2. Rotstein OD. Pathogenesis of multiple organ dysfunction syndrome: gut origin, protection, and decontamination. Surg Infect (Larchmt) 2000;1(3):217-23; discussion 223-5.

3. Pelsers MM, Hermens WT, Glatz JF. Fatty acid-binding proteins as plasma markers of tissue injury. Clin Chim Acta 2005;352(1-2):15-35.

4. Kanda $\mathrm{T}$, Nakatomi $\mathrm{Y}$, Ishikawa $\mathrm{H}$, et al. Intestinal fatty acid-binding protein as a sensitive marker of intestinal ischemia. Dig Dis Sci 1992;37(9):1362-7.

5. Lieberman JM, Sacchettini J, Marks C, Marks WH. Human intestinal fatty acid binding protein: report of an assay with studies in normal volunteers and intestinal ischemia. Surgery 1997;121(3):335-42.

6. Zeissig S, Burgel N, Gunzel D, et al. Changes in expression and distribution of claudin 2,5 and 8 lead to discontinuous tight junctions and barrier dysfunction in active Crohn's disease. Gut 2007;56(1):61-72.

7. Sackett DL, Haynes RB. The evidence base of clinical diagnosis. London: BMJ Publishing Group, 2002.

8. Crenn P, Coudray-Lucas C, Thuillier F, Cynober L, Messing B. Postabsorptive plasma citrulline concentration is a marker of absorptive enterocyte mass and intestinal failure in humans. Gastroenterology 2000;119(6):1496-505. 
9. Crenn P, Vahedi K, Lavergne-Slove A, Cynober L, Matuchansky C, Messing B. Plasma citrulline: A marker of enterocyte mass in villous atrophy-associated small bowel disease. Gastroenterology 2003;124(5):1210-9.

10. Blijlevens NM, van't Land B, Donnelly JP, M'Rabet L, de Pauw BE. Measuring mucosal damage induced by cytotoxic therapy. Support Care Cancer 2004;12(4):227-33.

11. Bossuyt PM, Lijmer JG, Mol BW. Randomised comparisons of medical tests: sometimes invalid, not always efficient. Lancet 2000;356(9244):1844-7.

12. Rowlands BJ, Soong CV, Gardiner KR. The gastrointestinal tract as a barrier in sepsis. $\mathrm{Br}$ Med Bull 1999;55(1):196-211.

13. Clark JA, Coopersmith CM. Intestinal crosstalk: a new paradigm for understanding the gut as the "motor" of critical illness. Shock 2007;28(4):384-93.

14. Clayburgh DR, Shen L, Turner JR. A porous defense: the leaky epithelial barrier in intestinal disease. Lab Invest 2004;84(3):282-91.

15. Lotze MT, Zeh HJ, Rubartelli A, et al. The grateful dead: damage-associated molecular pattern molecules and reduction/oxidation regulate immunity. Immunological Reviews 2007;220:60-81.

16. Fink MP, Delude RL. Epithelial barrier dysfunction: a unifying theme to explain the pathogenesis of multiple organ dysfunction at the cellular level. Crit Care Clin 2005;21(2):177-96.

17. Soeters PB, Luyer MD, Greve JW, Buurman WA. The significance of bowel permeability. Curr Opin Clin Nutr Metab Care 2007;10(5):632-8. 


\section{Chapter 6}

\section{Nederlandse samenvatting}

Dit proefschrift gaat ten eerste over het herkennen van darmziekten en ten tweede over de rol van de darm tijdens grote chirurgische ingrepen en bij ernstig zieke patiënten op de intensive care unit.

De voornaamste functie van de darm is de opname van voedingsstoffen. Daarom moet de darmwand doorlaatbaar zijn voor voeding, nodig voor energie en als bouwstof. De inname van voedsel gaat echter ook gepaard met de blootstelling van de darm aan een grote hoeveelheid lichaamsvreemde voedingsstoffen en micro-organismen. De tweede functie van de darm is dan ook om een effectieve barrière te vormen tegen deze potentieel schadelijke inhoud van de darm. Er bestaan vele mechanismen om deze barrière in stand te houden, welke besproken worden in hoofdstuk 1, paragraaf 3. Er zijn situaties waarin de barrière verstoord wordt en dit leidt dan vaak tot darmziekten en mogelijk tot andere gevaarlijke aandoeningen als bloedvergiftiging. Het is voor artsen soms lastig om vast te stellen of een darmziekte aanwezig is bij een patiënt die zich presenteert met buikklachten. Dit komt, doordat veel ziekten gepaard gaan met buikklachten, ook al is de darm er niet bij betrokken. Hierdoor is de diagnostiek van darmaandoeningen vertraagd en dientengevolgde ook de correcte behandeling, hetgeen leidt tot hogere morbiditeit en mortaliteit. Er is derhalve behoefte aan laboratoriumtesten die duidelijk maken of de darm beschadigd is. In aansluiting op deze diagnostische uitdaging, wordt de nabehandeling van darmziekten soms ook gehinderd door de afwezigheid van een niet-invasieve, snelle diagnostische test, die kan vaststellen of de therapie, gericht op herstel van de darmschade, succesvol is. In dit proefschrift werd een aantal potentiële testen geëvalueerd, die met name schade aan de darmcellen die de barrière vormen aantonen. Onderzoek naar markers voor myocardischemie gaf aanknopings-punten waaraan markers voor darmschade moesten voldoen. Troponine T is daarbij het meest treffende voorbeeld. Het is een klein eiwit dat karakteristiek voorkomt in het doelorgaan (hart) en snel de cel verlaat bij schade. Analoog hieraan komen in de darm eiwitten tot expressie, die behoren tot de familie van fatty acid binding proteins (FABP's). FABP's hebben normaliter een functie in het transcellulaire transport van langeketenvetzuren en galzouten, zijn in het cytosol gelokaliseerd, in water oplosbaar en hebben een laag moleculair gewicht (14-15 kDa). Deze eigenschappen zorgen ervoor dat ze de cel gemakkelijk verlaten na verlies van de integriteit van de celmembraan en terechtkomen in de circulatie. In de darm komen de isovormen intestinal (I)FABP, liver (L)-FABP en ileal-bile acid binding protein (I-BABP) voor. (Pilot)studies van andere onderzoekers wezen uit dat necrose van de darm vroegtijdig vastgesteld kan worden door in plasma en/of urine eiwitten te 
bepalen die afkomstig zijn uit het cytosol van darmepitheel. Om de plasma en/of urine concentraties van deze eiwitten te begrijpen, was inzicht nodig in de exacte lokatie van deze eiwitten in de darmwand. In hoofdstuk 2, paragraaf 2 wordt beschreven dat FABP's in de hele darm tot expressie komen en met name in de dunne darm. Microscopisch zijn deze eiwitten gelokaliseerd in de epitheelcellen. Bij patiënten met coeliakie, een ziekte die onder andere gekenmerkt wordt door epitheelschade door glutenovergevoeligheid, werd gevonden dat I-FABP en L-FABP concentraties in het bloed bruikbare markers zijn voor het vaststellen van met histologisch onderzoek geobjectiveerde darmschade. Ook bleken deze markers potentieel waardevol zijn in de evaluatie van het dieeteffect. Teneinde de klaringsdynamiek van FABP's te bepalen werd vervolgens peroperatief bij chirurgische patiënten bloed verzameld uit de aan- en afvoerende vaten van darmen, lever en nieren. Na analyse van pre- en postrenaal plasma werd vastgesteld dat FABP's door de nier werden geklaard (fractionele renale extractie: 28\%); berekend werd dat de plasma halfwaardetijd elf minuten bedroeg (hoofstuk 2, paragraaf 3).

Hieruit wordt geconcludeerd dat er sterke aanwijzingen zijn dat circulerend en urinair FABP's waardevolle markers zijn om darmschade aan te tonen.

Vervolgens werden deze testen gebruikt om zuigelingen, verdacht van de ernstige darmontsteking necrotiserende enterocolitis (NEC), vroegtijdig op te sporen. NEC komt voornamelijk voor bij te vroeg geboren, fles-gevoede kinderen. Deze aandoening kent een hoog sterftecijfer: 20-40\% van de kinderen met NEC overlijdt aan deze aandoening. Gebrek aan goede, betrouwbare (vroeg)diagnostische mogelijkheden is mede de oorzaak van de hoge mortaliteit en morbiditeit. Therapeutische modaliteiten worden daardoor te laat geïnitieerd. De belangrijkste bevinding is dat deze testen, die uitgevoerd kunnen worden in materiaal dat op niet-invasieve manier werd verkregen (te weten urine en faeces), in staat blijken om vast te stellen of zuigelingen die verdacht werden van NEC deze ziekte, daadwerkelijk hadden of niet (hoofdstuk 3, paragraaf 6 en 7). Dit is klinisch erg relevant, omdat zuigelingen zodoende voortaan sneller een adequatere behandeling kunnen krijgen.

Vervolgens werd onderzoek gedaan naar de hypothese, die voortkomt uit proefdierstudies en die stelt dat verlies van de darm barrière betrokken is bij het ontstaan van ontstekingsreacties, zoals kan plaatsvinden bij postoperatieve of posttraumatische complicaties, bloedvergiftiging (sepsis) en meervoudig orgaanfalen (multiple organ failure (MOF)). Deze proefdierstudies laten zien dat grote operaties of ernstig trauma leiden tot een verminderde doorbloeding van de darm, waarbij ontstekingsfactoren vrijkomen en de barrière van de 


\section{Chapter 6}

darmwand verloren gaat. Bovendien komen door celschade alarmsignalen uit het cytsoplasma van de cel vrij, die een ontstekingsrepons opwekken. Verlies van de darm barrière kan resulteren in de passage van schadelijke voedingsstoffen en micro-organismen en hun toxinen door de darmwand heen en het vrijkomen ervan in het lichaam. Dientengevolge kan een ongecontroleerde hevige ontstekingsreactie ontstaan, waardoor ziektebeelden als sepsis kunnen ontstaan. Voorgaand onderzoek naar bovenstaande hypothese in patiëntgebonden studies liet uiteenlopende resultaten zien. Daarom werd in dit proefschrift de vraag bestudeerd of de darm aangedaan is als gevolg van grote chirurgische ingrepen; de resultaten worden in hoofdstuk 4 gepresenteerd. Patiënten die op de intensive care unit terechtkwamen vanwege sepsis als complicatie na een grote buikoperatie bleken darmschade te hebben. Deze schade was gerelateerd aan de mate van doorbloeding van de darm. De hoeveelheid darmschade was een goede voorspeller voor de mortaliteit in deze groep patiënten. Twee studies waarin achtereenvolgens patiënten geïncludeerd werden die een langdurige operatie aan hun aorta of aan hun scoliose ondergingen, lieten zien dat darmschade en barrière verlies ontstond bij deze patiënten. De darmschade was gecorreleerd met doorbloedingsveranderingen van de darm en de erop volgende ontstekingsreactie.

Aangezien aangetoond werd dat de doorbloeding van de darm een belangrijke factor is voor de instandhouding van de barrière, werd een experimenteel model ontwikkeld waardoor de gevolgen van doorbloedingsstoornissen (ischaemie) van de darm in de mens bestudeerd konden worden (hoofdstuk 5). Daarenboven is darmischaemie een ziekte-entiteit, die ontstaat door thromboembolie, en die gepaard gaat met hoge morbiditeit en mortaliteit door de aspecifieke klinische presentatie en een gebrek aan accurate diagnostische mogelijkheden. In het nieuw ontwikkelde model voor darmischaemie kunnen derhalve ook nieuwe testen voor de vroegdiagnostiek van darmischaemie worden geevalueerd. In patiënten, die een pancreatico-duodenectomie ondergingen, werd gebruik gemaakt van het feit dat in deze procedure een variabele lengte dunne darm (jejunum) wordt verwijderd. Hierdoor ontstond een voor de patiënt risicoloos humaan jejunum ischaemie-reperfusie (IR) model. Geïsoleerd jejunum $(6 \mathrm{~cm})$ werd onderworpen aan 30 minuten ischaemie, gevolgd door reperfusie. Arterioveneuze concentratiegradiënten van I-FABP over bestudeerd jejunum liet het ontstaan van epitheliale celschade zien, die afhankelijk was van het mannosebindend lectine genotype van de patiënt. Direct na ischaemie was de intestinale epitheliale bekleding microscopisch intact, terwijl subepitheliale ruimtes ontstonden in de villus top door terugtrekking van de basaal membraan. Echter, na 25 minuten reperfusie werd massale afstoting van de mature epitheliale cellen in 
het lumen waargenomen, gepaard gaand met apoptose. Vervolgens was de epitheliale continuïteit hersteld binnen 60 minuten reperfusie en apoptose afwezig. Darmbarrièrefalen, gemeten door de concentraties van afbraakprodukten van bacteriën over het geïsoleerde stuk darm, en een ontstekingsreactie werden niet waargenomen. Concluderend; dit is de eerste humane studie die intestinale IR geïnduceerde celschade en de directe consequenties opheldert, waarin een uniek, endogeen beschermingsmechanisme beschadigde cellen elimineert en het ontstaan van barrièreverlies of inflammatie voorkomt. 


\section{Curriculum vitae}




\section{Curriculum vitae JPM Derikx}

Name: Joep Derikx

Christian names: Joseph Petrus Marie

Gender: Male

Date/Place of birth: June 5th 1978, Sittard

Marital status: happily lives together with Monique Pluijmen in Maastricht

E-mail: j.derikx@ah.unimaas.nl

\section{Education}

- Gymnasium, cum laude, Serviam (currently: Trevianum), Sittard: 19901996

- Medicine, University of Maastricht; class: 1996

- $\quad$ PhD-student, University of Maastricht (NUTRIM)/University Hospital Maastricht, department of surgery. Project: 'Compromised gut in neonates and children'. Supervisors: Prof. Dr. E Heineman and Prof. Dr. WA Buurman: 2003-2008

- $\quad$ Surgical resident, Maasland hospital Sittard (Head: Dr. AGM Hoofwijk): 2008 and Maastricht University Medical Centre+ (Head: Prof. Dr. MJHM Jacobs)

\section{List of publications}

- Derikx JP, van Waardenburg DA, Granzen B, van Bijnen AA, Heineman E, Buurman WA.

Detection of chemotherapy-induced enterocyte toxicity with circulating intestinal fatty acid binding protein.

J Pediatr Hematol Oncol. 2006;28:267-9.

- Derikx JP, De Backer A, van de Schoot L, Aronson DC, de Langen ZJ, van den Hoonaard TL, Bax NM, van der Staak F, van Heurn LW.

Factors associated with recurrence and metastasis in sacrococcygeal teratoma.

Br J Surg. 2006;93:1543-8.

- $\quad$ Derikx JP, De Backer A, van de Schoot L, Aronson DC, de Langen ZJ, van den Hoonaard TL, Bax NM, van der Staak F, van Heurn LW.

Long-term functional sequelae of sacrococcygeal teratoma: a national study in The Netherlands.

J Pediatr Surg. 2007;42:1122-6. 
- $\quad$ Derikx JP, Poeze M, van Bijnen AA, Buurman WA, Heineman E.

Evidence for intestinal and liver epithelial cell injury in the early phase of sepsis.

Shock. 2007;28:544-8.

- van de Poll MC, Derikx JP, Buurman WA, Peters WH, Roelofs HM, Wigmore SJ, Dejong $\mathrm{CH}$.

Liver manipulation causes hepatocyte injury and precedes systemic inflammation in patients undergoing liver resection.

World J Surg. 2007;31:2033-8.

- Derikx JP, Evennett NJ, Degraeuwe PL, Mulder TL, van Bijnen AA, van Heurn LW, Buurman WA, Heineman E.

Urine based detection of intestinal mucosal cell damage in neonates with suspected necrotising enterocolitis.

Gut. 2007;56:1473-5.

- Hanssen SJ, Derikx JP, Vermeulen Windsant IC, Heijmans JH, Koeppel TA, Schurink GW, Buurman WA, Jacobs MJ.

Visceral injury and systemic inflammation in patients undergoing extracorporeal circulation during aortic surgery.

Ann Surg. 2008;248:117-25.

- Derikx JP, Matthijsen RA, de Bruïne AP, van Bijnen AA, Heineman E, van Dam RM, Dejong $\mathrm{CH}$, Buurman WA.

Rapid reversal of human intestinal ischemia-reperfusion induced damage by shedding of injured enterocytes and reepithelialisation.

PLoS ONE. 2008;3:e3428.

- $\quad$ Thuijls G, de Haan JJ, Derikx JP, Daissormont I, Hadfoune M, Heineman E, Buurman WA.

Intestinal cytoskeleton degradation precedes tight junction loss following hemorrhagic shock.

Shock. 2009;31:164-9

- Luyer MD, Derikx JP, Beyaert R, Hadfoune M, Dejong CH, Heineman E, Buurman WA, Greve JW.

High-fat nutrition reduces IFN-gamma and decreases CpG-enhanced liver injury following hemorrhagic shock.

J Hepatol. 2009;40:342-50.

- Derikx JP, van Waardenburg DA, Thuijls G, Willigers HM, Koenraads M, van Bijnen AA, Heineman E, Poeze M, Ambergen T, van Ooij A, van Rhijn LW, Buurman WA

New insight in loss of gut barrier during major non-abdominal surgery PloS ONE. 2008;3:e3954. 
- Derikx JP, Blijlevens NM, Donnelly JP, Fujii H, Kanda T, van Bijnen AA, Heineman E, Buurman WA.

Loss of enterocyte mass is accompanied by diminished turnover of enterocytes after myeloablative therapy in haematopoietic stem-cell transplant recipients.

Ann Oncol. 2009;20:337-42.

- $\quad$ Bosch SF, Derikx JP, Thuijls G, van Bijnen AA, van Waardenburg DA, van Heurn, LW, Buurman WA, Heineman E

FABP's als nieuwe plasma- en urinemarkers voor intestinale epitheliale celschade.

Nederlands Tijdschrift voor Heelkunde. 2009;18:52-6.

- Derikx JP, Vreugdenhil AC, Van den Neucker AM, Grootjans J, van Bijnen AA, Damoiseaux JG, van Heurn LW, Heineman E, Buurman WA

A pilot study on the non-invasive evaluation of intestinal damage in celiac disease using I-FABP and L-FABP.

J Clin Gastroenterol, in press.

- Derikx JP, Bijker EM, Vos GD, van Bijnen AA, Heineman E, Buurman WA, van Waardenburg DA

Gut mucosal damage in the early phase of children with meningococcal sepsis.

Critical Care Medicine, in revision.

- $\quad$ Urine based Detection of Intestinal Tight Junction Loss.

Thuijls G, Derikx JP, J. de Haan J, J. Grootjans J, de Bruïne A, Masclee AAM, Heineman E, Buurman WA.

J Clin Gastroenterol, in press

- Mannose-binding lectin null alleles are associated with preserved epithelial cell integrity following intestinal ischemia reperfusion in man.

Matthijsen RA, Derikx JP, Steffensen R, van Dam RM, Dejong $\mathrm{CH}$, Buurman WA.

Molecular Immunology, in press.

- $\quad$ The localization of the LPS - recognition complex in the human healthy and inflamed premature and adult gut.

Wolfs TG, Derikx JP, Hodin CM, Driesen A, de Bruïne A, Bevins CL, Lasitschka F, Gassler N, van Gemert WG, Buurman WA.

Inflamm Bowel Dis, in press.

Prize

Pelerin prize for best young researcher, Maastricht University Medical Centre, 2008. 


\section{Grants}

- Maarten Kappelle Stichting, 'Gut wall integrity failure in prematures with necrotiscing enterocolitis': 2005.

- $\quad$ Stichting Jules Coenegracht sr., 'Chemotherapy-induced loss of gut wall integrity in children': 2005.

- $\quad$ AGIKO-stipendium 920-03-438, the Netherlands Organisation for Health Research and Development (ZonMw), 'Gut wall integrity loss in prematures with necrotising enterocolitis': 2006.

- $\quad$ Stichting Sint Annadal, 'Early diagnosis and follow-up of necotising enterocolitis: a joint study of the department of surgery and paediatrics of the University Hospital Maastricht': 2007. 
Geen enkele (ouder van een) patiënt heeft geweigerd om deel te nemen aan een van de studies, beschreven in dit proefschrift, terwijl we geen enkele patiënt direct konden helpen met de onderzoeken. De reden die alle (ouders van) patiënten gaven om toch mee te doen aan de studies was zonder uitzondering: 'Het onderzoek is niet schadelijk voor mij en ik kan er in de toekomst toch veel mensen in een zelfde positie als de mijne mee van dienst zijn.' Ongelooflijk veel dank ben ik verschuldigd aan de patiënten (en hun ouders).

Mijn promotores Erik Heineman en Wim Buurman wil ik van harte danken voor de kans die ze me hebben gegeven om het onderzoek naar 'Compromised gut in neonates and children' mee op te starten, voor de begeleiding en haast onvoorwaardelijke steun. Erik, jouw enthousiasme en positivisme hebben me gestimuleerd om zo veel mogelijk te doen. Wim, jij hebt me onder andere geleerd om klinische wetenschap met een basale blik tegemoet te treden. Ook wil ik Simone en Jeanette, de echtgenotes van mijn promotores, bedanken voor hun hartelijke ontvangst in Cadier en Keer en Eijsden tijdens de vele besprekingen.

Ernst, jou dank ik voor de gelegenheid die je me gaf om het Nederlandse na-onderzoek te doen naar patiënten geopereerd aan een sacrococcygeaal teratoom en om de resultaten te presenteren op menig congres. Daarna heb je me geholpen om de begeerde promotieplek te krijgen bij de (kinder)chirurgie. Ook tijdens mijn promotie hebben we een aantal studies samen kunnen doen.

De andere Mestreechter (kinder)chirurgen wil ik bedanken voor hun bijdrage en belangstelling: Dr Sie (ook voor het leuke congresbezoek in Turijn en Istanbul), Dr Van Gemert, Dr Verhoeven.

Daarnaast wil ik de kinderartsen en neonatologen bedanken voor hun inzet om van de gemeenschappelijke studies een succes te maken. Met name Prof. Zimmerman, Dr. Mulder, Dr. Degraeuwe, Dr. Kramer, Dr. Van der Hoeven, Dr. Moonen en Dr. Van Waardenburg, Dr. Van den Neucker, Dr. Vreugdenhil en Dr. Granzen waren nauw betrokken bij onze studies.

Kees Dejong en Ronald van Dam, dankzij jullie konden de Chef en ik erg gaaf onderzoek doen naar darmischaemie. Prof. Greve, Dr. Bemelmans, Jan en Antje: dank dat jullie ons altijd altijd zo enthousiast welkom heetten als we op de operatiekamer kwamen voor deze studie.

Martijn Poeze wil ik bedanken voor de ondersteuning om snel een artikel voor elkaar te krijgen. Daarnaast wil ik de Nijmeegese darm-geïnteresseerden Nicole Blijlevens en Peter Donnelly bedanken voor het mooie stuk dat we hebben gemaakt. I want to thank Dr. Fujii and Dr. Kanda for their help in 


\section{Dankwoord}

constructing (anti-)I-BABP. Prof. De Bruïne wil ik danken, omdat we altijd binnen mochten lopen met nieuwe coupes en voor zijn betrokkenheid bij het darmischaemie-stuk. Dr. Van Rhijn, Dr. Van Ooij, Dr. Willigers en Dick van Waardenburg wil ik danken voor het hechte team dat we vormden wat betreft de scoliose-studie. Verder dank aan alle andere mede-auteurs op de stukken.

Dan kon dit onderzoek ook niet plaats hebben gevonden zonder de steun van de verpleegkundigen van de NICU (eerst onder leiding van Mark, later van Jos) en PICU. Gelukkig hebben jullie onze studies vanaf het begin warm ontvangen en jullie betrokken gevoeld. Ook wil ik de operatie-asistenten en anaesthesiemedewerkers bedanken voor hun medewerking als we weer eens met een kar of apparaat de operatiekamer op kwamen. Jullie toonden zonder uitzondering interesse in het onderzoek.

De beoordelingscommissie dank ik voor het beoordelen van dit proefschrift.

Toen ik op het lab kwam, wist ik niet hoe te pipetteren, hoe een computer werkte, wat een PCR/ELISA/IHC/WB is, hoe een paper te submitten, hoe een koffiezetapparaat werkte en wat te doen met alle vrije tijd. Gelukkig werd ik daarbij goed en vakkundig geholpen door enkele doorgewinterde collegae. Ik heb lang getwijfeld of ik echte namen ga noemen in het dankwoord of de namen, zoals ze op het lab in zwang zijn. Ik heb besloten beide eens op te schrijven, zodat vast ligt wie nu precies wie is. Dit zal voor veel collegae handig zijn, aangezien een enkeling nog steeds niet weet of Hans nu Bas is of dat Bas Hans is, wie nu Ruud of Tim is en of er een verschil is tussen Sjeng, Chef en Sjaak.

De voorgangers die me de goede weg wezen, wil ik danken: Bart (de Vriescoupekoning), Marcel, Yvonne (Yf), Misha (DJ Daiper, Dr Luyer). Robert (eerst Koffert, later de Chef) wil ik danken voor de mooie tijd: menig mooi tochtje op de fiets, naar congres en Arhus; samen begonnen, samen klaar! Sjeng (Tim, Prof. Tip, de PCR-koning) wil ik danken voor de gelijkenis op Erwin, André Kuijpers en Gaston. Bij vrijwel alles wat op het lab gebeurt, ben je betrokken en we hebben als buurmannen veel leuks meegemaakt: oa skitrips, voetbalpartijtjes en nog wat experimenten, waarvoor heel veel dank! Dan komt daar een van de grootste steunpilaren van het lab: Mo. Hadfoune, wat een mannetje ben je ook: een uitgesproken mening, die je ook ter discussie wilt stellen, gekoppeld aan een schaterlach waar iedereen vanzelf vrolijk van wordt. Dank aan Geertje (kortweg Geert) voor de vele brainstormsessies over onze 
studies, het uitvoeren van veel werk dat we samen bedachten en voor de aangename congresbezoekjes. Dank ook aan Joepie (de Direkteur): ik was blij dat je op mijn mail reageerde en al vroeg bij ons op het lab onderzoek kwam doen en bent gebleven! Je bent daardoor snel een vaste waarde geworden met een grote nieuwsgierigheid naar alles wat op en rondom het lab plaatsvindt. Tim (Ruud) wil ik danken voor de Top(pers)-muziek die hij heeft gedraaid in de gaafste, succesvolste en mannelijkste kamer van het lab. Tine, dank dat je de kar zo voortvarend hebt getrokken van de NEC-studie op de NICU. Jacco-Juri (Sjaak), leuk je nu ook als co-assistent en collega te kennen. Ook Kim (Kimmerd) wil ik danken voor de gezelligheid en voor het beheren van de agenda van Wim (voor zover dat mogelijk is). Daarbij wil ik dan meteen Anouk, Nicole en Sandra, de secretaresses van Erik, danken, die altijd een gaatje vrijmaakten in zijn agenda als ik wilde langskomen. Dan wil ik ook de rest van de voormalige collegae bedanken: Trudi, Sarah (Rinsy), Kees, Jeroen (Nijhuisman), Sylvia, Martien, Nicole, Nicholas, Bas (Hans, zeker ook voor het fraaie stuk over TAAA), Hans (HPLC-Hans), Dennis (v/d Ven) en Dennis (Jaap), Loes, Kirsten, Marcella, Paul, Gabri, Mariëlle, Yvette, Mick, Albert, Ayhan, Bart(je Boterzuur), Iris, Femke, Yanti, Sander (Rens), Froukje (Goukje, Sjoukje), Caroline (Houdini), Dian (Dianski), Kaatje, Johanne, Kim, Maarten, Eva, Ruben, Maartje, Nina, Kirsten. Dank aan alle studenten, die hebben bijgedragen aan dit proefschrift: Bregje, Fredje, Arianne, Sofie, Job\&Rinske, Caroline/Tine, Sander, Kim, Joepie, Marianne, Vera/Caroline, Else. Lest, best: Annemarie, zonder jouw kennis en kunde waren onze studies onmogelijk geweest. Ongelooflijk veel dank ben ik je verschuldigd voor het consciëntieus uitvoeren en analyseren van de experimenten, voor het ordenen van alle samples en voor het soepele, ongecompliceerde team dat we vormden.

Dank aan de pulmo's (onze buren, mn Ramon, Harry, Jos en Juanita) voor de vele team-buildingsmomenten van ons lab waar jullie graag lijdend voorwerp wilden zijn.

De assistenten en chirurgen uit Sittard bedank ik voor de goede, warme na-onderzoekse opvang. Ik vond het spannend om aan de opleiding te beginnen. Het team waarin ik terecht ben gekomen, zorgt ervoor dat ik me als een vis in het water voel, ongelooflijk veel leer en met veel plezier naar ons mooie ziekenhuis kom.

Alle mannetjes van het creatieve, slimme, lompe en sportieve dispuut Rusticus wil ik danken voor de onvergetelijke studententijd in Maastricht, 


\section{Dankwoord}

gehucht aan de Maas, stad van Hogeschool Maastricht, stad van Universiteit Maastricht, stad van de Inkom! In het bijzonder wil ik danken de mannen van het eerste uur (en even daarna): Martijn (10us), Jasper, Raph (de Sambaschurk), Tim (de Haas Jansen, 16 jaar), Marc (de pauw/rups); TSS10a/JSD(\&P): Stijn (de PPP), Dussie, Pier(lalalaaa) en als laatste de leden van EUR: Stijn, Jacco-Juri (Sjaak), Laurent (Stobbe), Rolf (Koning Loch), en af en toe Joepie (Praloupie). Gao staon ajje veur Rusticus bin!

Dank ook aan Léon en Alexander van fietsclub SMS en hun vrouwen voor de inspannende ontspannen trainingsritjes op zondagochtend.

Ik wil de leden van kook- en eetclub 't Klaoske bedanken voor de topavonden. Klaske en Joost (vaste deel van de kookclub): ik heb veel bewondering voor de maaltijden die jullie altijd weer van overal ter wereld op tafel toveren, die Marieke\&Dave en Monique\&ik (vaste deel van de eetclub) dan weer met veel verwondering opeten.

Alles heb ik natuurlijk te danken aan mijn familie. Ook al lijk je je vaak weg te cijferen voor de rest van ons gezin, toch ben jij het middelpunt en degene die ons bindt, papa. Met veel trots zien jij, Max en ik dat we uit hetzelfde hout zijn gesneden. Samen met mama heb jij altijd het klimaat geschapen waarin Max en ik konden doen wat we wilden. Jullie hebben ons de weg gewezen, en later heb je met Marianne ons los gelaten, maar we zijn elkaar nooit kwijt geraakt. Dat komt vast doordat we elke dag geprobeerd hebben het gouden randje te vinden, zoals jij en mama dat aan Max en mij geleerd hebben. Ik ben blij dat jij, Marianne, weer zorgde voor uitbreiding van ons gezin door met Alexander en Dikkie bij ons te komen wonen. Niet alleen papa bloeide daarvan op en heeft er veel van geleerd. Dan natuurlijk ook veel dank aan mijn broertje, Max, en zijn Jo voor alle interesse, steun en de gezellige weekendjes Breda/Haarlem/Maastricht. Ook Alexander, Dikkie en Jona dank voor jullie interesse. Ook de rest van de femieje dank ik: omes, tantes, naefkes en nichskes: Zitterd allein! De sjweierfamielje oes Kirchrao zijn mij en Monique op veel momenten en veel manieren tot steun geweest en hebben altijd met veel zorg bijgedragen aan ons geluk: veel dank! Daarbij wil ik meteen ook Maurice en Yvette danken voor hun oprechte (en gedeelde) interesse en alle leuke bezoekjes, boekjes en stukken vlaai. Als laatst wil ik hier melden dat ik erg benieuwd ben naar de feestelijke kleding van Peggy en naar de mening van Ronald over het boekje en de promotie. Dank dat jullie met zijn tweeën van dichtbij betrokken zijn geweest bij mijn doen en laten door de jaren heen. 
'My smile is my make-up, I wear since my wake-up with you'. Lieve Monique, Pluijmpje, duizend maal dank voor je liefde, zorgzaamheid en gesjnep, die me zo blij, gelukkig en vrolijk maken.

Kirchröadsjer meadsjer, kiek ze diech aa.

Kiek wie ze laache, ze laache diech aa.

Hant muulsjere, die zint 't puutsje wead, hant beks-jere mollieg en ronk.

Ze zint vuur tse klauwe, zoeë wie me dat zeat, zoeë meadsje dat maat diech jezonk. 
Covidien

Greiner Bio-One

Danone

Johnson \& Johnson

B Braun

Hycult Biotechnology

Stichting Jules Coenegracht sr.

AGIKO-stipendium 920-03-438, the Netherlands Organisation for Health Research and Development (ZonMw)

Stichting Sint Annadal

MaatschapHeelkunde Orbis Medisch Centrum Sittard 
